\title{
CATALOGOS
}

$\rightarrow$ DH $r$

FAUNA BRAZILEIRA

EDITADOS PELO

MUSEU PAULISTA

S. PHULO - BRAZIL

\section{Vol. II}

\section{OS MYRIAPODOS DO BRAZIL}

POR

HENRY W. BRÖLEMANY

Ex-Presidente da Soc. Entomologique de France

Correspundente do Museum d'Hist. Nat. de Paris

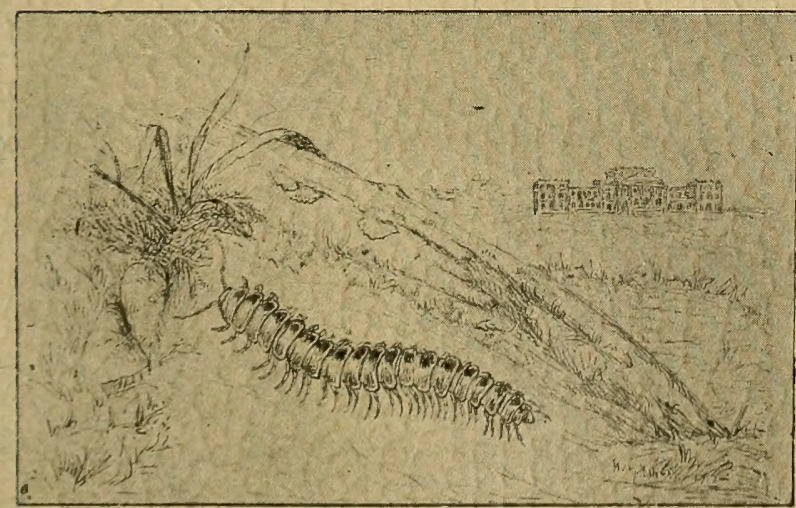

SÃO PAULO

TYP, CRRDOZO, FILHO \& C.-R, Direita, 35.

1909 



\section{CATALOGOS}

\section{DA ra \\ FAUNA BRAZILEIRA \\ EDITADOS PELO}

MUSEU PAULISTA.

S. PAULO - BRTZIL

\section{Vol. II}

\section{OS MYRIAPODOS DO BRAZIL}

POR

HENRY W. BRÖLEMANN

Ex-Presidente da Soc. Entomologique de France

Correspondente do Museum d'Hist. Nat. de Paris

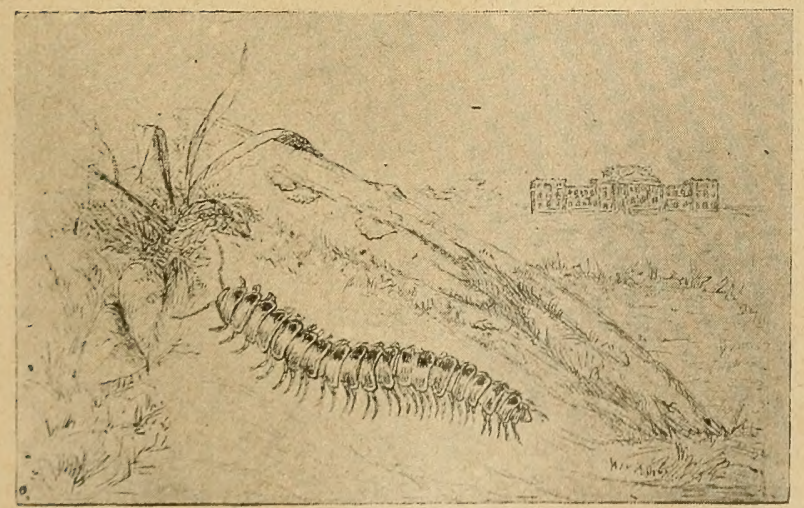

SÃO PAULO

TYP, CARDOZO, FILHO \& C. $-R$, Direita, 35. 



\section{Essai d'un Catalogue}

DES

\section{MYRIAPODES DU BRÉSIL}

PHR

\section{HENRY W. BRÖLEMANN}

Ancien Président de la Soc. Entomologique de France

Correspondant du Museum d'Hist. Nat. de Paris

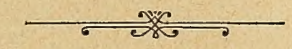

VOI. II

DOS

CATALOGOS DA FAUNA BRAZILEIRA

EDITADOS PELO

\section{MUSEU PAULISTA \\ S. PAULO-BRAZIL}

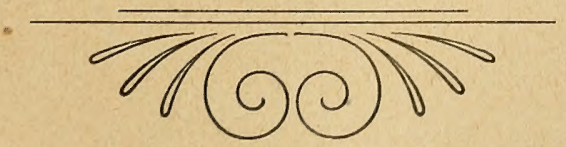





\section{NDEX}

Introduction . . . . . . . . . . p. I

Bibliographie . . . . . . . . . . . p. III

Index systematique. . . . . . . . . . . p. XXI

Les Myriapodes du Brésil . . . . . . . . p. 3

Addenda. . . . . . . . . . . . . . p. 84

Index alphabetique . . . . . . . . . . p. 87

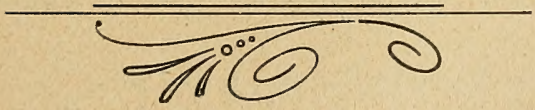





\section{ESSAI D'UN CATALOGUE \\ - DES - \\ MYRIAPODES DU BRÉSIL \\ PAR}

\section{БENIRY W. BRÖLEMANN}

Ancien Président de la Soc. Entomologique de France

Correspondant du Museum d'Hist. Nat. de Paris

La classification adoptée dans ce travail est la suivante:

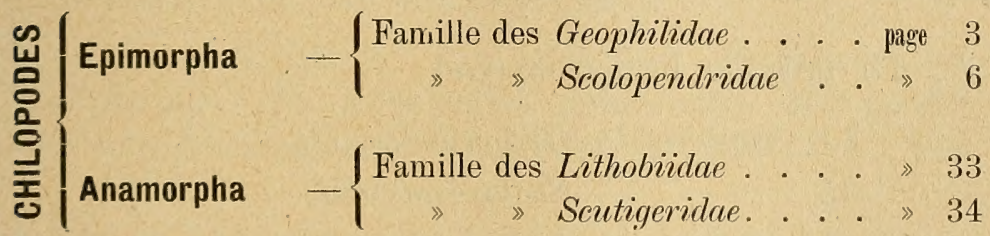

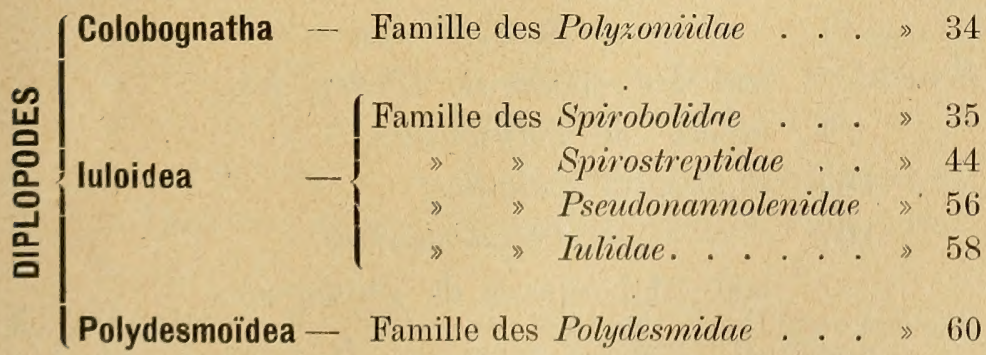

Les genres d'une même famille sont classés par ordre alphabétique; les espèces d'un même genre sunt classés dans le même ordre. 
Les indications bibliographiques sont composées du nom d'auteur suivi d'un numero auquel est souvent adjoint une lettre (par ex.: Bollman $N^{\circ} .88 \mathrm{c}$ ); le numero est fourni par les deux derniers chiffres de la date de publication de l'ouvrage visé (dans l'espèce: 1888); lorsque plusieurs travaux d'un même auteur ont été publiés dans la même année, il a été ajouté une lettre (dans l'espèce: $c$ ) pour les distinguer les uns des autres.

Toutes les synonymies figurent dans le catalogue à leur place alphabétique, avec renvoi au nom d'espèce valable. Exception a été faite pour celles des synonymies de la Scolopendra morsitans et de la Scolopendra subspinipes qui n'ont pas été citées pour le Brésil; ces synonymies étant extrémement nombreuses, leur adjonction au catalogue n'aurait eu d'autre résultat que de l'allonger, sans lui donner plus d'intérêt ou d'utilité pratique.

Seules les espèces supposées valables sont accompagnées d'un numero d'ordre.

La présente liste comprend:

11 espèces de Geophilides,

35 - - Scolopendrides,

1 espèce de Lithobiides,

1 - Scutigerides,

soit 48 espèces de Chilopodes,

1 espèce de Polyzonides,

104 espèces de Iuloüdes,

107 - Polydesmö̈des, soit 212 espèces de Diplopodes,

260 noms d'espèces réputées valables, au total.

Cannes, le 15 Décembre 1902. 


\section{BIBLIOGRAPHIE}

C. Attems N. 97 a - Myriopoden. Abh. d. Senckenberg. naturhist. Gesell. Bd. XXIII, Heft III, Frankfurt a/M. 1897.

C. Attems $\mathbf{N}^{\circ} 98$ a - Myriopoden aus Semon, Zoologische Forschungsreisen in Australien und dem Malayischen Archipel. Iena. Denkschr., Bd. VIII, Iena. 1898.

C. Attems $\mathbf{N}^{\circ} 98$ b - - System der Polydesmiden, $1^{\text {ter }}$ Theil. Denkschr. K. Akad. Wiss., Math.-Naturw. Cl., Bd. LXVII, 1898.

C. Attems $\mathbf{N}{ }^{\circ} 99$ b - System der Polydesmiden, $2^{\text {ter }}$ Theil. Ibid. Bd. LXVIII, 1899.

C. Attems $\mathbf{N}{ }^{\circ} \mathbf{0 0} \mathbf{b}$.- in Therese, Prinzessin von Bayern: In Südamerica gesammelte Myriapoden und Arachnoïden - Zool. Anz., Bd.XXIII, N. 615 , 1900 .

C. Attems $\mathbf{N} 0^{\circ} 01$ c - Neue Polydesmiden, des Hamburger Museums. Mitth. a.d. Naturhist. Museum, XVIII, (2 ${ }^{\text {tes }}$ Beihft. z. Jairb. d. Hamb. Wissensch. Anst. XVIII), Hamburg. 1901, p. 85-108.

C. Attems N. 01 d -.. Neue, durch den Schiffsserkehr in Hamburg eingeschleppte Myriopoden. Ibid. 19̄01, p. 111-116.

E. Blanchard N. 49 - in Cuvier; Le Régne animal, distribué d'après son organisation. 3" Ed. Paris, 1836-1849.

C. H. Bollman N. $87 \mathrm{~g}-\mathrm{New}$ North-American Myriapods. Entomol. Americ., III, 1887. 
C. H. Bollman $\mathbf{N}$. $^{\circ} 88$ a - A preliminary list of the Myriapoda of Arkansas, with descriptions of new species, 1bid., IV, 1888.

C. H. Bollman N. 88 - Notes upon a Collection of Myriapoda fron East-Tennessee, with deacription of a new genus and six new species. Ann. N. York Acad. Sci. 1888.

C. H. Bollman N. $88 \mathrm{e}$ - Notes on a collection of Myriapoda from Cuba. Proc. U. S. Nat. Mus., XI, 1888, p. 335-338.

C. H. Bollman $\mathbf{N} .^{\circ} \mathbf{8 8} \mathbf{d}-$ Notes on a collection of Myriapoda fron Mossey-Creek, Tenn., with a description of a new species. Ibid. 1888, p. 339-342.

C. H. Bollman N. ${ }^{\circ} 88$ f -- Notes upon some Myriapods belonging to the U. S. National Museum. Ibid. 1888 , p. 343-35€.

C. H. Bollman N. $88 \mathbf{g}$ - Catalogue of the Myriapods of Indiana. Ibid. 1888, p. 403-410.

C. H. Bollman N. ${ }^{\circ 9}$ - Myriapoda. Sbid., XII, 1889.

C. H. Bollman N. 93 - The Myriapoda of NorthAmerica - Pothumous Work edited by L. M. Underwood, containing the above mentioned notes together with Bollman's pusthumous writings. Bull. U. S. Nat. Mus. N. 46 Washington. 1893.

I. F. Brandt $\mathbf{N}^{\circ} 31$ - De nova Insectorum multipedum seu myriapodum familia Pentazoniorum (s. Glomeridiorum ) nomine designanda. Bull. des Mem. de l'Acad. Sci. St. Petersbourg, 6.e ser., Sci. math. phys., II, 1833.

I. F. Brandt N. ${ }^{\circ} \mathbf{3 3}$ - Tentaminum quorumdam monographicorum Insecta Myriapoda Chilognatha Latrellii spectantium. Bull. Soc. Imp. Natural. Moscou, VI, 1833. 
I. F. Brandt N ${ }^{\circ} 39$ a - Note relative à la classification des espèces qui composent le genre Polydesmus, et suivie d'une caracteristique de dix espèces nouvelles, ainsi que de quelques remarques sur la distribution géographique des espèces en général. Bull.Sci.Acad. St. Petersbourg, V, 1839 (Recueil, 5).

I. F. Brandt $\mathbf{N}^{\circ} \mathbf{4 0}$ b - Observations sur les esèpces qui composent le genre Scolopendra, suivies de caractères des espèces, qui se trouvent dans le $\mathrm{Mu}$ seum Zool. de l'Acad, des Sci. de St. Peterbg., et quelques coups d'oeil sur leur distribution géographique. Ibid, VII, 1840. (Recueil, 2).

I. F. Brandt $\mathbf{N}^{\circ} \mathbf{4 0}$ c - Remarques générales sur l'ordre des Insectes Myriapodes. Ibid. VII, 1840. (Recueil, 1).

I. F. Brandt N. 40 d - Generis Iuli specierum enumeratio, adjectis plurium, quae hucusque nondum ennotuerunt specierum brevibus descriptionibus ad Musei Academiae Scientiarum Petropolitanae specimina factis. Ibid. VIII, 1840. (Recueil, 4).

I. F. Brandt $\mathbf{N}^{\circ} \mathbf{4 0}$ e - Note supplémentaire sur quelques espèces du genre des Scolopendres, suivie de la description de deux espèces nouvelles et d'un essai d'une subdivision de ce genre en deux sousgenres. Ibid. VIII, 1848. (Recucil, 3 ).

I. F. Brandt. N..$^{\circ} \mathbf{1 1}$ d - Recueil de mémoires relatifs à l'ordre des Insectes Myriapodes. Extrait des Bull. Scientif. Acad. Imp. Sci. de St. Petersboury, V, VI, VII, VIII, et IX. St. Petersbourg et Leipzig. 1841.

H. W. Brölemann N." 95 c - Elenco di Miriapodi raccolti in Lombardia. Boll. Soc. Entom. Ital., XXVII. Firenze, 1895. 
H. W. Brölemann N. $\mathbf{9 6}^{\circ}$ e - Myriapodes recueillis dans les serres du Muséum. Bull. Mus. Hist. Nat., N. 1, Paris, 1896.

H. W. Brolemann N. 96 d - Mission scientifique de Mr. Ch. Alluaud aux Iles Séchelles (Mars, Avril, Mai 1892)-Mrriapodes. Mem. Soc. Zool. France, VIII, 1895.

H. W. Brölemann $\mathbf{N}^{0} \mathbf{9 6} \mathbf{h}$ - Liste de Myriapodes des Etats Unis, et principalement de la Caroline du Nod, faisant partie des collections de Mr. Eugène Simon. Ann. Soc. Entom. France, LXV, 1896.

H. W. Brölemann N. ${ }^{0} \mathbf{9 7}$ b - Myriapodes recueillis à l'Ile Madère par M. A. Fauvel en 1896. Bull. Soc. Entom. Franie, N. $7,1897$.

H. W. Brölemann N. 97 d -- Un mystérieux myriapode-Scolopendropsis bahiensis Brandt. Bull. Soc. Zool. France, XXII, N. ${ }^{\circ}$ 5-6, 1897.

H. W. Brölemann N. 98 e-Voyage de M. ${ }^{r}$ E. Si-

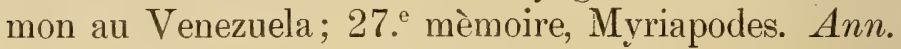
Soc. Entom. France. LXVII. 1898.

H. W. Brölemann N. ${ }^{\circ} 98 \mathbf{t}$-Myriapodes du Haut et Bas Sarare (Venezuela) donnés par M. ${ }^{r}$ F. Geay au Muséum d'Histoire Naturelle de Paris. Ibid. $189 \dot{8}$.

H. W. Brölemann N. ${ }^{0} 00$ a-Dous Myriapodos notaveis do Brazil; Notas Myriapodologicas. Bol. do Mus. Paraense. III. N. ${ }^{1}$ 1. 1900 (cf. Zooi. Anz. XXIII, N.0 612. 1900).

H. W. Brölemann N. $\mathbf{0}^{\circ} \mathbf{0 0} \mathrm{g}-$ Myriapodes d'Amérique. Mem. Soc. Zool. France XIII. 1900.

H. W. Brölemann N. 01 e-Les Myriapodes du Musée de São Paulo. Rev. do Mus. Paul. V. 1901.

H. W. Brölemann N. 02 e-Myriapodes recueillis par M. ${ }^{r}$ E. Gounelle au Brésil. Ann. Soc. Entom. France. LXXI. 1902. 
H. W. Brölemann N. $02 \mathbf{f}$-- Myriapodes recueillis au Pará par M. ${ }^{r}$ le Prof. E. A. Goeldi, Directeur du Musée Goeldi. Zool. Anz. 1903.

H. W. Brölemann N. 03 a - Myriapodes du Musée de São Paulo; II. memoire, Manáos. Rev. do Mus. Paul. VI. 1905.

A. G. Butler N. 76-Preliminary notice of new species of Mrriapoda and Arachnida from Rodriguez. Ann. \& Mag. Nat. Hist., 4 ser. XVII. 1876.

0. F. Cook N. $95 \mathbf{f}$--Priodesmus, a new genus of Diplopoda from Surinam. Proc. U. S. Nat. Mus. XVIII, N. ${ }^{\circ} 1037.1895$.

0. F. Cook N. ${ }^{\circ} 96 \mathbf{c}-\mathrm{A}$ new Diplopod Fauna in Liberia. Amer. Natur: 1896.

0. F. Cook N. ${ }^{\circ}$ 96 e VII-Brandtia-A series of occasional papers on Diplopoda. Huntington, N. Y. 1896.-VII; The genera of Oryidae.

E. Daday de Dées $\mathbf{N} .^{\circ} 89$ a-Myriapoda Regui Hungariae. E commissione regiae Societ. hung. Scient. natur. Budapest. 1889.

E. Daday de Dées N. 89 c-Myriapoda extıanea Museae nationalis hungarici. Term. Fuz. XII. 1889.

E. Daday de Dées $\mathbf{N} .^{\circ} \mathbf{9 1}$ - Ausländische Myriopoden der Zoologischen Collection der Universität zu Heidelberg. Term. Fuz. XIV. 1891.

E. Daday de Dées $\mathbf{N} .^{\circ} \mathbf{9 3}$ - Myridpoda extranea nova vel minus cognita in Collectione Musaei nationalis hungarici. Term. Fuz. XVI. 1893.

C. De Geer N. ${ }^{78}$ - Mémoires pour servir à l'histrire des Insectes'; 9." minoire. VII. Stockholm. 1778.

E. Ellingsen N." 92 -- Birbag til Kundskaben om de Norske Myriopoders Udlinedelse. Christian. Vidensk. Selsk. Forh. N. ${ }^{\circ}$ 10. 1891. 
1. F. Eschscholtz. N. 23 - Animalia tetracera et Myriapoda exotica. Mem. Soc. Natur. Moscou. VI. 1823.

I. Fabricius N. ${ }^{\circ 5}$ - Systema entomologiae. Flensburg. 1775 .

I. Fabricius $\mathbf{N}{ }^{\circ} \mathbf{8 1}-$ Species insectorum. I. Hamb. et Kiloni. 1781.

I. Fabricius N. ${ }^{\circ} \mathbf{8 7}$ - Mantissa insectorum, sistens eorum species nuper detectas. I. Hafniae. 1787.

I. Fabricius N. 93 -- Entomologia systematica, emendata et aucta. II. Hafniae. 1793.

Ed. Fleutiau N. 01 -- Sur un Arthropode ennemi de nos serres. Pull. du Jard. Colon. 1901.

C. E. A. Gerstäcker No $\mathbf{7 3}-$ Gliederthierfauna des Zanzibar-Gebietes. Die Myriopoden in: Claus v. d. Decken's Reisen in Ostafrika. III; ¿̈. ${ }^{\text {te }}$ Abth. Leipz. u. Heidelb. 1873.

P. Gervais N. 36 b - Note sur le Genre Polydesmus de la classe des Myriapodes. Ann. Soc. Entom. France. V. 1836.

P. Gervais N. 37 a - Etudes pour servir à l'Histoire Naturelle des Myriapodes. Ann. Sci. Nat. VII. 1837.

P. Gervais N. 41 - in Eydoux \& Souleyet.--Voyage de la Bonite. Zoologie; Insectes aptères. 1841.

P. Gervais N. $\mathbf{4 4} \mathbf{d}$-Descriptions de trois espèces de Scolopendres; sur le genre Cambala. Ann. Soc. Entom. France (Bulletin). 2. ser., II. 1844.

P. Gervais N. 47 a - in Walckenaer. - Histoire Naturelle des Insectes aptères. IV. Paris. 1847.

P. Gervais $\mathbf{N} .^{\circ} \mathbf{5 9}$ - in F. de Castelnau. - Expédition dans les parties centrales de l'Amérique du Sud. VII. Paris. 1859. 
Gervais of Goudot N. ${ }^{\circ} 4$ - Description des Myriapodes recueillis por I. Goudot en Colombie. Ann.

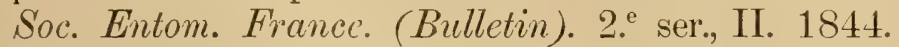

C. G. Giebel $\mathbf{N}^{\circ} \mathbf{7 0}$ - Ein neuer Iulus vom Amazonenstrom. Zeitschr. f. gesam. Naturw. XXXV. 1870.

E. A. Goeldi N. ${ }^{0}$ - Os Myriapodos do Brazil (Embuás e Centopeias). Bol. Mus. Paraense, I, N. 2, 1895.

I. E. Gray N. 44 - List of the specimens of Myriapoda in the collection of the British Museum. Printed by order of the Trustees, London. 1844.

E. Haase N. ${ }^{\circ} \mathbf{8 7 6}$ - Die Indisch-Australischen Myriopoden; I, Chilopoden. Abh. u. Ber. kön. zool. antrop. etnogr. Mus. Dresden, N. ${ }^{\circ}$ 5. Berlin. 1887.

A. Humbert N. ${ }^{\circ}$ 55 - Essai sur les Myriapodes de Ceylan. Mem. Soc. Phys. Hist. Nat. Genève, XVIII, 1865.

A. Humbert \& H. de Saussure N. 69 a -.-Description de divers Myriapodes du Musée de Vienne. Verh. d. Zool. Bot. Gesells. Wien, XIX, 1869.

A. Humbert \& H: de Saussure N. 69 b--Myriaporla nova americana. Revue \& Mag. de Zool. 2." ser. XXI, 1869.

A. Humbert \& H. de Saussure N. 70-Myriapoda nova americana. Ibid., XXII, 1870.

A. Humbert \& H. de Saussure N. 72-Etudes sur les Myriapodes. - Mission scientifique au Mexique et dans l'Amérique Centrale.-.-Zoologie, VI; 2." section. Paris, 1872.

If von thering $\mathbf{N}^{\circ} 95-$ (Bibliographia, p. 24:3) Revista do Museu Paulista, I, 1895.

F. Karsch N. 80 b - U(t)er die von I)r. Finsch während seiner polynesischen Reise gesammelten My- 
riopoden und Arachniden. Sitzgsber: Gesells. naturf. Freunde, Berlin, N. 5, 1880.

F. Karsch N. ${ }^{\circ} \mathbf{8 1}$ a - Verzeichnis der während der Rohlfschen afrikanischen Expedition erbeuteten Myriopoden und Arachniden. Troschel, Arch. f. Naturgesch. XLVII, 1 heft, 1881, p. 1-18.

F. Karseh N. 81 c -- Zum Studium der Myriapoda Polydesmia. Ibid. 1881. p. 36-49.

F. Karsch $\mathbf{N}^{\circ} \mathbf{8 1} \mathbf{d}$-- Arachniden und Myriopoden Mikronesiens. Dewitz, Berliner Entom. Zeitsch., XXV, 1881.

F. Karsch $\mathbf{N} .^{\circ} \mathbf{8 1} \mathbf{g}-$ - Neue Iuliden des Berliner $\mathbf{M u -}$ seums als Prodromus einer Iuliden Monographie. Zeitsch.f. ges. Naturw., 3. to Folge, VI (LIV). 1881.

F. Karsch $\mathbf{N}{ }^{\circ} \mathbf{8 4}$ b-Ueber einige neue und minder bekannte Arthropoden des Bremer Museums. Abh. Nath. Ver. Bremen. IX. 1884.

F. Karsch N. ${ }^{\circ} 88$ a - Zwei neue Myriopoden von Ecuador. Berl. Entom. Zeitsch. XXXII, 1 Heft. 1 1 888.

C. Koch N. ${ }^{\circ}$ 41---Arachniden und Myriapoden aus der Regentschaft Algier. In Wagner's Reisen in der Regentschaft Algier. III. Leipzig. 1841.

C. Koch N. 47-System der Myriapoden. Drei Bändchen zu (Panzer) Herrich-Schäffer: Kritische Revision der Insectenfauna Deutschlands. Regenburg. 1847.

C. Koch N. 63 - Die Myriapoden getreu nach der Natur abgebildet und beschrieben. Halle 1863.

L. Koch N. 62 - Die Myriapoden-Gattung Lithobius. Nürnberg. 1862.

L. Koch N. ${ }^{0}$ 65-Beschreibung neuer Arachniden und Myriapoden. Verhandl. Zool.-Bot. Gesells. Wien, XV. 1865. 


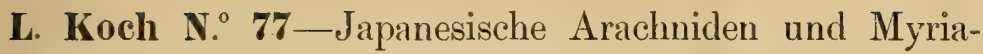
poden. Ibid. XXVII. 1877.

E. Kolhrausch N. ${ }^{\circ}$ 78--Beiträge zur Kenntnis der Scolopendriden. Dissertation. Marburg. 1878.

E. Kolhrausch N. ${ }^{\circ} \mathbf{8 1}$ - Gattungen und Arten der Scolopendriden. Arch. f. Naturg. v. Troschel, XLVII. 1881.

P. E. Latreille N. ${ }^{\circ}$ 02--Histoire Naturelle des Crustacés et des Insectes. Paris. 1802-1804 (an X et an XII).

R. Latzel N. 84 a - Die Myriapoden der Oesterreichisch-Ungarischen Monarchie. II. Wien 1884.

R. Latzel N. ${ }^{\circ} 95$ a-- Myriopoden aus der Umgebung Hamburgs. Beiheft z. Jahrb. Hamb. Wissensch. Anstalten, XII. 1895.

R. Latzel N. 95 b-Beiträge zur Kenntniss der $\mathrm{My}$ riopodenfauna von Madeira, den Selvages und den Canarischen Inseln. Ibid. 1895.

W. E. Leach N. ${ }^{\circ}$ 14-A tabular view of the external characters of four classes of Animals, which Linné arranged under Insecta. Trans. Lin. Soc. London, XI. 1814-1815.

W. E. Leach $\mathbf{N}^{\circ} \mathbf{1 7}$-The zoological Miscellany. London. 1817.

A. Lendl N. ${ }^{\circ}$ 94-Myriapoda et Arachnida Asiae Orientalis. Ex Op. Széchenyi Iter adoras Aside orientalis. Budapest. 1894.

Linné N. ${ }^{\circ} 58$ - Systema naturae. Ed. X. Holmiae. 1758.

Linné N. 66 - Systema naturae. Ed. XII. Holmiae. 1766 .

Lister (Plumier) N. 99-A jonrney to Paris in the year 1698. VIII. London. 1699. 
H. Lucas $\mathbf{N}^{\circ} 39$ a-In Webb \& Berthelot: Histoire Naturelle des Arachnides, Myriapodes et Thysanoures des Iles Canaries. Paris. 1839.

H. Lucas N. 40 b-In Blanchard: Histoíre Naturelle des animaux articulés. (Hist. Nat. des Crust., des Arachn. et des Myriap. I.) Paris. 1840.

H. Lucas $N^{\circ}{ }^{\circ} 49$ c-Exploration scientifique de l'Algérie. Zoologie. Paris. 1849.

H. Lucas N. ${ }^{\circ} 84$-Description d'une nouvelle espèce de Scolopendre. Bull. Soc. Entom. France. 6. ${ }^{\text {e }}$ ser., IV. 1884.

I. M. ${ }^{\circ}$ Neill N. ${ }^{\circ} \mathbf{8 7}$ a - List of the Myriapods found in Escambia County, Th., with description of six new species. Proc. U. S. Nat. Mus. X. 1887.

I. M. ${ }^{\circ}$ Neill $\mathbf{N}{ }^{\circ} 88-\mathrm{A}$ list, with brief descriptions or all the species, including one new to science, or Myriapoda of Franklin Co., Ind. Bull. Brookville Soc., Nat. Hist. N. 3. 1888.

G. $^{\text {ta }}$ Mariani N. ${ }^{\circ} 00$ - Sulla Fauna di Serra. Boll. $d$. Naturalista. XX, N. 8 . Siens. 1900.

F. S. Mattozo N. ${ }^{\circ} \mathbf{8 1}$ - Les Myriapodes d'Afrique au Museum de Lisbonne. Journal Sc. Acad. Lisboa. 1881.

Fr. Meinert N. $\mathbf{7 0}^{-}$Myriapoda Musei Havniensis. Bidrag til Myriapodernes Morphologie og Systematik. I, Geophili. Naturh. Tidsskr. af Schiodte. 3 r., VII. 1870-71.

Fr. Meinert N. 84 b-Myriapoda Musei Havniensis. J3idrag ec. III, Chilopoda. Vidensk. Meddel. Naturh. Foren. Kjöbenhavn. 1884-86.

Fr. Meinert $\mathbf{N}^{\circ} \mathbf{8 5}$ - Myriapoda Musei Cantabrigenis, Mass.; Part I, Chilopoda. Proc. Amer. Philos. Soc. XXIII. 1886. 
Mikan N. 34 - Ueber die in Brasilien gesammelten Iulus-Arten. Oken's Isis. 1834.

G. Newport N. 44 a-A list of the species of Myriapoda, order Chilopoda, contained in the Cabinets of the British Museum, with synoptic descriptions of forty-seven new species. Ann. Mag. Nat. Hist., first ser., XIII. 1844. p. 94-101.

G. Newport $N{ }^{\circ} \mathbf{4 4} \mathbf{b}-\mathrm{A}$ list of the species of Myriapoda, order Chilognatha, contained in the Cabinets of the British Museum, with descriptions of a new Genus and thirty-two new Species. Ibid. 1844. p. 263-270.

G. Newport N. 44 -M-Monograph of the class Myriapoda, order Chilopoda; with Observations on the General Arrangement of the Articulata. Trans. Lin. Soc. London. XIX. 1844.

G. Newport \& I. Gray $\mathbf{N} .^{0} 56$-Catalogue of the $\mathrm{My}$ riapoda in the collection of the British Museum. I, Chilopoda. London. 1856.

A. G. Olivier N. 92-Histoire Naturelle des Insectes. Encyclopédie méthodique. VIII. Paris. 1792.

M. Perty N. ${ }^{\circ} 33$--Delectus animalium articulatorum, quae collegit Spix et Martius. Monachae. 1833.

W. C. H. Peters $\mathbf{N}{ }^{\circ} 55$ - Ueber die Myriapoden im Allgerneinen und insbesondere über die in Mossamhique beobachteten Arten. Monatsber. $K$. preuss. Ak. d. Wiss. Berlin. XXIX. 1855.

W. C. II. Peters N." 62--Wissenschaftliche Reise nach Mrssambique. V. Zoologie. Berlin. 1862.

W. C. H. Peters N. 64-Uebersicht der im Kön. zool. Museum befindlichen Myriopoden aus der Familie der Polydesmi, so wie Beschreibung einer neuen Gattung, Trachyiulus, der Iuli und neuer Arten 
der Gattung Siphonophora. Monatsber, K. preuss. Akad. Wiss. Berlin. 1864. Iuli.--Supplement, Ibd. 1864. September-October.

R. I. Pocock N. ${ }^{\circ} 88$ a--Description of Scolopendra valida Lucas, with notes on allied species. Ann. Mag. Nat. Hist. (6), II. 1888 p. 335-340.

R. I. Pocock N. ${ }^{\circ} 88$ c-Contributions to our knowledge of the Myriapoda of Dominica. Ibid. 1888. p. $472-483$.

R. I. Pocock N. 88 e-Report on the Myriapoda of the Mergui Archipelago, collected for the Trustees of the Indian Museum, Calcutta, by Dr. John Anderson. Journ. Lin. Soc. London, Zool. XXI, N. ${ }^{\circ} 132.1889$.

IR. I. Pocock $\mathbf{N} .^{\circ} 90 \mathbf{d}-\mathbf{A}$ short account of a small collection of Myriapoda obtained by M. Edw. Whimper in the Andes of Ecuador. Ann. Mag. Nat. Hist. (6), VI, 1890.

R. I. Pocock N. 91 b - Notes on the synonymy of some species of Scolopendridae with descriptions of new Genera and Species of the Group. Ann. Mag. Nat. Hisi. (6), VI, 1891.

R. I. Pocock N. 91 c - Descriptions of some (11) new species of Chilopoda. Ibid. (6), VIII, 1891.

R. I. Pocock N. 91 e - On the Myriapoda of Burma. Part. II. Report upon the Chilopoda collected by Sig. L. Fea and M. ${ }^{r}$ E. W. Oates. Ann. Mus. Civ. Stor. Nat. Genova. (2), X (XXX). 1891.

R. I. Pocock N. 92 b - Report upon two collections of Myriapoda sent from Ceylon by Mr. E. E. Green and from various parts of Southern India by Mr. Edgar Thurston, of the Government Central Museum, Madras. Journ. Bombay Nat. Hist. Soc. VII. 1892. 
R. I. Pocock N. 92 c -- Supplementary notes on the Arachnida and Myriapoda of the Mergui Archipelago; with descriptions of some new Species from Siam and Malaysia. Journ. Lin. Soc. London. XXIV, N. ${ }^{\circ}$ 153. 1892.

R. I. Pocock N. ${ }^{\circ} 93$ a - Report upon the Myriapoda of the "Challenger" Expedition, with remarks upon the Fauna of Bermuda. Ann. Mag. Nat. Hist. (6), XI. 1893. p. 121-142.

R. I. Pocock N. ${ }^{\circ} 93 \mathbf{b}$ - Upon the Identity of some of the Types of Diplopoda contained in the collection of the British Museum, together with descriptions of some (6) new Species of exotic Iulidae. Ibint. 1893. p. 248-254.

R. I. Pocock N. $93 \mathbf{c}-$ On the Myriapoda of Burma. Part III. Report upon the Iulidae, Chordeumidae and Polyzonidae, collected by Sig. L. Fea and Mr. E. W. Oates. Ann. Mus. Civ. Stor. Nat. Genova. (2), XIII (XXXIII). 1893.

R. I. Pocock N. 93 d - Contributions to our knowledge of the Arthropod-Fauna of the West-Jndies. Part II, Chilopoda. Jours. Lin. Soc. London, Zool. XXIV, N.o 146,1893 p. 454-473.

R. I. Pocock N. 93 e - Contributions to our knowledge of the Arthropod-Fauna of the West-Indies. Part III. Diplopoda \& Malacopoda. Ibid. 1893. p. $473-544$.

R. I. Pocock N. 94 b - Chilopoda, Symphyla and Diplopoda from the Malay Archipelago. In: Zoologische Ergebnisse einer Reise in Niederländisch Ost-Indien (Max Weber), III, 2 ${ }^{\text {to }}$ Heft. 1894.

R. I. Pocock N. 95 a - Report upon the Chiloporla and IDiploporla obtained hy P. W. Bassett-Smith and J. I. Walker during the cruise in the Chinese 
Seas of H. M. S. "Penguin", Com. ${ }^{\text {der }}$ W. N. Moore commanding. Ann. Mag. Nat. Hist. (6) XV, 1895.

R. I. Pocock N. 95 c -- The Myriapoda of Burma. Part IV, Report upon the Polydesmoidea collected by Sig. L. Fea and Mr. E. W. Oatas. Ann. Mus. Civ. Stor. Nat. Genova. (2), XIV (XXXIV). 1895.

R. I. Pocock N. ${ }^{\circ} 95$ h - Biologia Centrali Americana 1895.

R. I. Pocock N. 96 e - Report upon the Scorpions, Spiders, Centipedes and Millipedes obtained by M. and M. ${ }^{\text {rs }}$ Lort Phillips in the Goolis Mountains inland of Berbera, N. Somailland. Ann. Mag. Nat. Hist. (6) XVIII, 1896.

R. I. Pocock N. 98 a - On the Arachnida and Myriapoda obtained in Funafuti by Prof.o W. I. Sollas and M.r Stanley-Gardiner, and in Rotuma by M.r Stanley-Gardiner. Ann. Mag. Nat. Hist. (7) I, 1898.

R. I. Pocock N. $00 \mathrm{~d}$ - Christmas Island Monograph. Chilopoda, Diplopoda and Arachnida. 1900.

R. I. Pocock N. 01 c - The Chilopoda or Centipedes of the Australian Continent. Ibid. (7) VIII, 1901.

C. O. von Porat N. 71 - Myriapoda Africae Australis in Museo regio Holmiensis asservata. Pars I, Chilopoda. Ofvers. K. Sv. Vetensk. Akad. Törh. N. 9 , XXVIII, 1871.

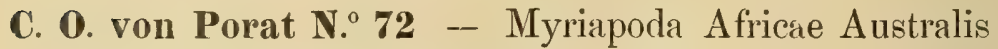
in Museo regio Holmiensis asservata. Pars II, Ibid. N. 5, XXIX, 1873.

C. O. von Porat $\mathbf{N} .^{\circ} \mathbf{7 6}-$ Om nagra exotiska Myriapoder. Bihang till K. Sv. Vetensk. Akad. Handl. N. ${ }^{7}$, IV, 1876. 
C. 0. von Porat $\mathbf{N}^{\circ} \mathbf{8 8}$ b - Ueber einige exotische Iuliden des Brüsseler Museums. Ann. Soc. Entorn. Belg. XXXII, 1888.

C. O. von Porat $\mathbf{N}^{\circ} \mathbf{8 9}-$ Nya Bidrag till Skandinaviska Halföns Myriapodologi. Entom. Tidskr. 10 Erg. 1-3 Heft. 1889.

C. O. von Porat N. 94 - Zur Myriopodenfauna Kamerun's. Bih. till. K. Sv. Vetensk. Akad. Handl. N. $5, \mathrm{XX}, 1894$.

W. I. Rainbow No ${ }^{\circ} 97$ - The Insect-Fauna of Funafuti: in Atoll of Funafuti. Austr. Mus. Mem. III 1897.

R. de la Sagra $\mathbf{N}{ }^{\circ} 56$ - Historia fisica politica y natural de la Isla de Cuba, VII, 1856.

H. de Saussure $\mathbf{N}{ }^{\circ} \mathbf{5 9}$ - Note sur la Famille des Polydesmides, principalement au point de vue des espèces américaines. - Diagnoses de divers Myriapodes noveaux. Linnaea Entomol. XIII. 1859.

II. de Saussure N. 60 - Essai d'une faune de Mrriapodes du Mexique avec la description de quelques espèces des autres parties de l'Amérique. (Deuxième Mémoire des "Mémoires pour servir à l'Histoire Naturelle du Mexique, des Antilles et des Etats Unis"). Mem. Soc. Phys. Hist. Nat. Genève. XV. 1860.

Eh. Say N. 21 - Description of the Myriapoda of the U. S. Journ. Acad. Nat. Sci. Philada. First ser, II. 1821.

Eh. Say N. 22 -- Oeuvres entomologiques, publiées par Lequien fils. 1822.

F. Silvestri N. 94 e - Chiloporli e Diplopodi della PaMusia. Ann. Mus. Civ. Stor. Nat. Genona, (2), XIV, (XXXIV). 1894. 
F. Silvestri N. ${ }^{\circ} 95$ b - I Chilopodi e i Diplopodi di Sumatra e delle isole Nias, Engano e Mentarei. Ibid., (2), XIV, (XXXIV), 1895.

F. Silvestri N. ${ }^{\circ} 95$ e - Chilopodi e Diplopodi raccolti dal Capitano G. Bove e dal Prof. L. Balzan nell'America meridionale. Ibid. 1895.

F. Silvestri N. 95 d - Viaggio del Dott. Alfredo Borelli nella Republica Argentina e nel Paraguay. Chilopodi e Diplopodi. - Boll. Mus. Zool. e Anat. comp. R. Univ. Torino. X. N. 23. 1895.

F. Silvestri N. 95 f - Esplorazione del Giuba e dei suoi affluenti compiuta dal Capitano V. Bottego durante gli anni 1892-1893 sotto gli auspicii della Soc. Geografica Italiana. - Risultati zoologici XVI, Chilopodi e Diplopodi. Ann. Mus. Civ. Stor. Nat. Genova. (2), XV (XXXV). 1895.

F. Silvestri $\mathbf{N}{ }^{\circ} 96$ b - Diplopodi di Borneo. 1bid. (2), XVI (XXXVI). 1896.

F. Silvestri N. ${ }^{\circ} 96 \mathbf{c}$ - Chilopodi e Diplopodi raccolti dal Dott. E. Festa a La Guayra, nel Darien e a Cuenca. Boll. Mus. Zool. Anat. comp. R. Univ. Torino. XI. N. ${ }^{\circ}$ 254. 1896.

F. Silvestri $\mathbf{N}{ }^{\circ} 96 \mathrm{~g}$ - Chilopodi e Diplopodi raccolti da Don Eugenio dei Principi Ruspoli durante l'ultimo suo viaggio nelle regioni dei Somali e dei Galla. Ann. Mus. Civ. Stor. Nat. Genova. (2), XVII (XXXVII). 1896.

F. Silvestri N ${ }^{\circ} 97$ a - Descrizione di una nuova famiglia di Diplopodi del Messico. Boll. Mus. Zool. Anat. comp. R. Univ. Torino. XII. N.o 277. 1897.

F. Silvestri N. ${ }^{\circ} 97$ b -- Viaggio del Dott. Alfredo Borelli nel Chaco Boliviano e nella Republica Argentina. Ibid. XII. N. ${ }^{\circ}$ 283. 1897. 
F. Silvestri N. ${ }^{\circ} 97$ e - Neue Diplopoden. Abh. u. Ber. K. Zool. Anthrop. Ethnogr. Mus. Dresden. VI. N.o 9. 1897.

F. Silvestri N. ${ }^{\circ} 97$ e - Description des espèces nouvelles de Mvriapodes du Musée Royal d'Histoire Naturelle de Bruxelles. Ann. Soc. Entom. Belg. XLI. 1897.

F. Silvestri $\mathbf{N} .^{\circ} 97 \mathbf{g}$ - Chilopodi e Diplopodi raccolti dal Cap. ${ }^{\text {"o }}$ V. Bottego durante il suo secondo viaggio nelle regioni dei Somali e dei Galla. Ann. Mus. Civ. Nat. Genova. (2), XVII (XXXVII). 1897.

F. Silvestri N. $98 \mathbf{k}$-Chilopodi e Diplopordi dell'ultima spedizione Bottego. Ibid. (2), IX (XXXIX) 1898.

V. Storm N. 98 -Myriopoda \& Oniscoida ved Throndhjem. Kgl. Norske Vidensk. Sels. Skrif. N. 8. 1898.

0. Tömösvary N." 85-Myriapoda a Joanne Xantus in Asia Orientali collecta enumeravit speciesque novas descripsit (opus postumum). Term. Fuz. IX. 1885.

L. M. Underwood $\mathbf{N}{ }^{\circ} \mathbf{8 7}$-The Scolopendridae of the United States. Entomol. Americ. III. N. 4. 1887.

C. Verhoeff N. ${ }^{\circ} 97$ c-Diplopoden Rheinpreussens und Beiträge zur Biologie und vergleichenden Faunistik europäischer Diplopoden; Vorläufer zu einer rheinpreussischen Diplopedenfauna. Verh. Naturh. Ver. preuss. Rheinl. LIII. Jahrg. 1897.

C. Verhoeff $\mathbf{N}{ }^{\circ} 01 \mathrm{f}$-Beiträge zur Kenntnis palaearktischer Myriopoden. VIII. ${ }^{\text {ter }}$ Aufsatz: über Diplopoden aus Süddeutschland und Tirol. Jhareshef. Ver. vaterl. Naturkunde in Wiütlbg. LVII. 1901.

E. Voges N." 78 a-Beiträge zur Kenntinss der Iuliden. Zeitsceler. f. wissensch. Zool. von Sicbold u. Rö̈ll. XXXI. 1878. 
H. C. Wood N. 61 - Descriptions of new species of Scolopendra in the Collection of the Academy. Proc. Acad. Nat. Sci. Philad. 1861.

H. C. Wood N. ${ }^{\circ}$ 63-On the Chilopoda of N.-America with a Catalogue of all the specimens in the collection of the Smithsonian Institution. Journ. Acad. Nat. Sci. Philad. new ser., V. 1863.

H. C. Wood N. ${ }^{\circ} 65$ a-The Myriapoda of North-America. Trans. Amer. Philos. Soc., new ser., XIII. 1869.

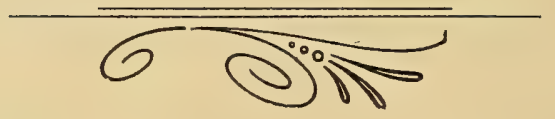




\section{INDEX SYSTEMATIQUE}

CLASSE

\section{Myriapoda-Chilopoda}

Ord. EpIMorpHa

Fam. Geophilidae

Aphilodon

Chomatobius

Geophilus

Heniorya

Notiphilides

Orphnaeus

Schendyla

Fiam. Scolopendridae

Cormocephalus

Cryptops

Cupipes

Newportia

Otocryptops

Otostigmus

Rhysida

Scolopendra

Scolopendropsis

Scolopocriptops

Ord. Anamorpha

Fam. Lithobiidae

Lithobius

Fam. Scutigeridae

Scutigera

CLASSE

\section{Myriapoda-Diplopoda}

Oro. Colonognatha
Fam. Polyzonidae

3

3

3

3

3

4

4

4

5

6

6

Fam. Pseudonannolenidae

Siphonotus

ORd. Juloidea $\quad 35$

Fam. Spirobölidae 35

Paraspirobolus $\quad 35$

Rhinocricus $\quad 35$

Spirobolellus $\quad 39$

Spirobolus $\quad 39$

Trigoniulus 44

Fam. Spirostreptidae 44

Archispirostreptus $\quad 44$

Spirocyclistus $\quad 45$

Spirostreptus $\quad 45$

Pseudunannolene $\quad 56$

Fam. Iulidae

Tulus $\quad 58$

Ord. Polvdesmoïdea 60

Fam. Polydesmidae $\quad 60$

Cryptodesmus $\quad 60$

Eurydesmus $\quad 61$

Euryurus 61

Fontaria $\quad 62$

Katantodesmus $\quad 62$

Leptodesmus $\quad 63$

Odontotropis 74

Orthomorpha 74

Platyırhacus $\quad 77$

Polydesmus $\quad 77$

Priodesmus 81

Strongylosoma $\quad x^{\prime 2}$ 



\section{MYRIAPODA - CHILOPODA}

\section{EPIMORPHA}

1. ${ }^{\text {er }}$ Fam. GEOPHILIDAE

1. Ger Gre: APHILODON Silvestri, 1898.

1. Aphilodon micronyx Brölemanı, 1901.

Aphilodon micronyx, Brölemann N.o $01 \mathrm{e}^{(1)}$. Hab.: (1) Brésil.

Mus. Paul.: Brésil (Type).

2. Gerve: CHOMATOBIUS Humbert \& Saussure, 1870.

2. Chomatobius brasilianus Humbert de Satssure, 1870.

Chomatobiu: brasilianus, Humb. \& Sauss. N. 70, $72^{(1 .)}$

(I)'aprìs Pocock- N." 95 h- cette espèce serait synonyme de Orphnaens brasiliensis Meinert.)

Hab.: (1) Brésil, Rio Negro. 


\section{3.e Genre: GEOPHILUS Leach, 1814.}

3. Geophilus guillemini Gervais, 1847.

Geophilus guillemini, Gervais N. $47 \mathrm{a}^{(1)}, 59^{(1)}$.

Hab.: (1) Brésil.

Newport \& Gray N. $56^{(1)}$.

4. Geophilus sublaevis Meinort, 1870.

Geophilus sublaevis, Meinert N. $70^{(1)}$. Hab.: (1) Brésil (Lagoa-Santa).

4. Genie: HENIORYA Cook, 1896.

5. Heniorya longissima Cook, 1896.

Heniorya longissima, Cook N. 96 e VII ${ }^{(1)}$. Hab.: (1) Brésil.

Nannophilus brasilianus = Schendyla brasiliana.

\subsection{Genre: NOTIPHILIDES Latzel, 1880.}

6. Notiphilides grandis Brölemani, 1903.

Notiphilides grandis, Brölemann N.: 03 a ${ }^{(1)}$. Hab.: (1) Manáos. Mus. Paul.: Est. Amazonas, Manáos (Type). 
6. Genre: ORPHNAEUS Meinert, 1870.

7. Orphnaeus brasiliensis Meinert, 1870.

Orphnaeus brasitiensis, Meinert N. $70^{(1)}, 85^{(1.6 . \overline{7})}$. Bollman N. $88 \mathrm{c}^{(8)}$.

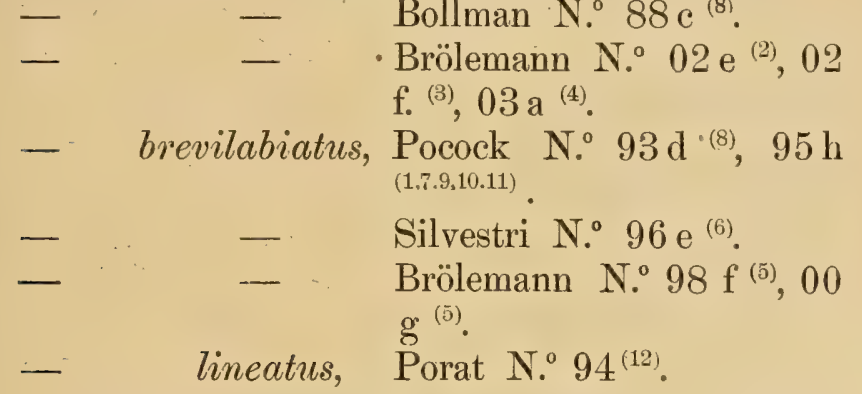

An syn.?: Orphraeus brevilabiatus Newport et auct.

(Pocock-N. 95 h-met en synonymie Orphnaeus brasiliensis avec Orphnaeus brevilabiatus. Il semble cependant que la question ne soit pas absolumeut tranchée.-Porat- No 94-considère $O$. brasiliensis comme synonyme de $O$. lineatus Newport; si cette interprétation est exacte, c'est ce dernier nom qui doit prévaloir comme ayant la priorité).
Hab.: (1) Brésil
2) Pernambuco.-
(3) Pará. -
Manáos. -
5) Venezuela.-
(6) Colombie. --
Honduras. -
(8) Antilles. -
(9) Panama. (10) meroon.
(11) Mexique.- ${ }^{(12)}$ Afrique, Ka-

Mus. Paul.: Est. Amazonas, Manáos.

Orphnaeus brevilabiatus -... Orphnaeus brasiliensis.

Orphnacus lineatus = Orphndeus brasiliensis. 
7. Genre: SCHENDYLA Bergsoe og Meinert, 1866.

8. Schendyla brasiliana (Siliestri), 1897.

Nannophilus brasilianus, Silvestri N. $97 \mathrm{e}^{(1)}$ Hab.: ${ }^{(1)}$ Brésil.

9. Schendyla gounellei Brölemanu, 1902.

Schendyla Gouncllei, Brölemann N.o $02 \mathrm{e}^{(1)}$. Hab.: (1) Brésil; Fazenda Nova Nicaragua (S.P.).

10. Schendyla imperfossa Brölemann, 1901.

Schendyla imperfossa, Brölemann N.00 $01 \mathrm{e}^{(1)}$. Hah.: (1) Brésil.

Mus. Paul.: Brésil (? ubi) (Type).

11. Schendyla paulista Biölemann, 1903.

Schendyla paulista, Brölemann N.o 03 a (1). Hab.: (1) Brésil ; Poço Grande (S.P.). Mus. Paul.: Est. S. Paulo, Poço Grande (Type).

\section{Fam SCOLOPENDRIDAE}

Branchiostoma affine = Rhysida longipes. Branchiostoma celer = Rhysida celeris. Branchiostoma gracile = Rhysida longipes. Branchiostoma longipes - Rhysida longipes Branchiostoma obsoletum $=$ Rhysida longipes. Branchiostoma scabricauda = Otostigmus scabricauda. 
8. Gemre: CORMOCEPHALUS Newport, 1844.

12. Cormocephalus aurantiipes Nerport, 1844.

Cormocephalus aurantiipes, Newport N. $44 \mathrm{c}^{(5)}$.

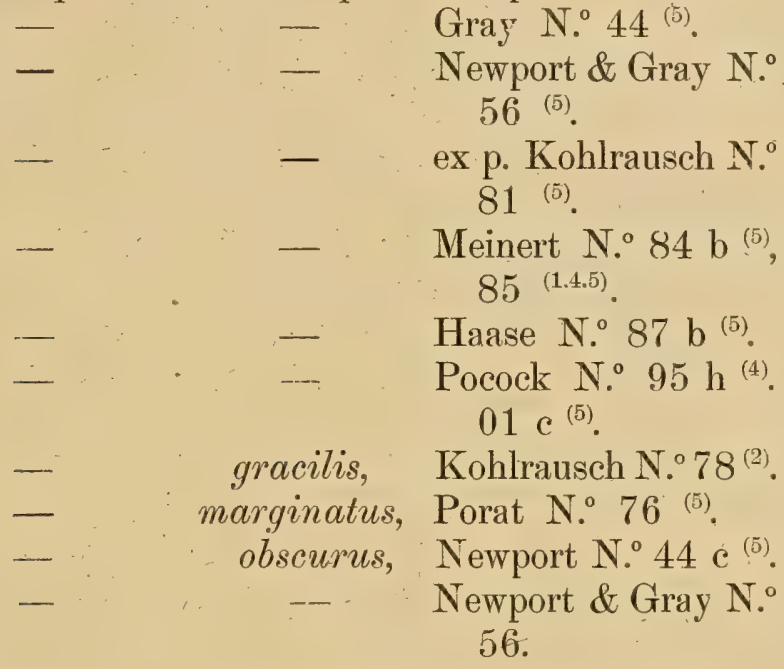

pygomelas, Kohlrausch N. 81
Scolopendia aurantiipes, Newport N: 44 a

Gervais N. 47 a $^{(5)}$.

- obscura, Gervais N." 47 a.

- puncticeps, Gervais N. 47 a ${ }^{(5)}$.

Non syn.: Cormocephalus aurantiipes ex p., Kohlrausch.

(Voir l'observation de Pocock- N. 95 h-au sujet de cette espèce.)

Hab.: (1) Rio de Janeiro._- (2) Surd-Amérique? _ (3)

Arnérique australe.- ${ }^{(4)}$ Guatemala. - - ${ }^{(5)}$ Australie.

Cormocephalus brasilicresis - Cupipes brasiliensis. Cormocephalus gracilis - Cormocephalus aurantiipes. Cormocephalus marginatus Cormocephalus aurantiipes. 
Cormocephalus obscurus $=$ Cormocephalus aurantiipes.

Cormocephalus puncticeps $=$ Cormocephalus aurantiipes.

Cormocephalus pygomelas = Cormocephalus aurantiipes.

9. Genre: CRYPTOPS Leach, 1814.

13. Cryptops brasiliensis Attems, 1901.

Cryptops brasiliensis, Attems $\mathrm{N}^{\circ} 01 \mathrm{~d}^{(1)}$. Hab.: (1) Brésil; Rio Grande do Sul.

14. Cryptops capivarae Pocock, 1891.

Cryptops capivarae, Pocock N.o $91 \mathrm{e}^{(1)}$. Hab.: (1) Brésil ; Rio Capivari.

15. Cryptops iheringi Brölemann, 1901.

Cryptops iheringi, Brölemann N.$^{\circ} 01 \mathrm{e}^{(1)}$. Hab.: (1) Brésil; Alto da Serra (Type). Mus. Paul.: Est. S. Paulo, Alto da Srrrra (S. P.). Cryptops sexspinosa seu sexspinosus = Otocryptops sexspinosus.

10. Genre: CUPIPES Kohlrausch, 1878.

16. Cupipes brasiliensis (Humbert \& Saussure), 1870.

Cormocephalus brasiliensis, Humb. \& Sauss. N.c $70,72^{(1)}$.

Cupipes brasiliensis, Kohlrausch N.0 $78^{(1)}, 81^{(1)}$. Hab.: (1) Brésil. 
17. Cupipes ungulatus Meinert, 1885.

Cupipes ungulatus,

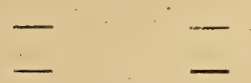

$-$
Meinert $\mathrm{N} .{ }^{\circ} 84 \mathrm{~b}^{(3.7) \text {, }}$ $85^{(1.4 .5 .7)}$.

Pocock N. 93 d ${ }^{(6)}$. Silvestri N. 95 c ${ }^{(3)}$, $97 \mathrm{~b}^{(4.5)}$.

Brölemann, N. $03 \mathrm{a}^{\left({ }^{(2)}\right.}$.

Var: mitis Brölemann, 1903.

Cupipes ungulatus mitis, Brölemann N. 03 a ${ }^{(2)}$. Hab.: (1) Pernambuco.-_(2) Manáos._-( ${ }^{(3)}$ Paraguay. ${ }^{(4)}$ Argentine._- ${ }^{(5)}$ Bolivie.- ${ }^{(6)}$ Antilles._- ${ }^{(7)}$ Haïti. Mus. Paul. : Est. Amazonas, Manáos; var. mitis Est. Amazonas, Manáos (Type).

\section{Genre: NEWPORTIA Gervais, 1847.}

18. Newportia (Scolopendrides) amazonica Brölemann, 1903.

Newportia (Scolopendrides) amazonica, Brölemann, Hab.: (1) Manáos. N. 03 a ${ }^{(1)}$. Mus. Paul.: Est. Amazonas, Manáos (Type).

19. Newportia aurantiaca Gervais, 1847.

Scolopocryptops aurantiaca, Gervais N. 47 a ${ }^{(1)}$, $59^{\text {(1) }}$

Newportia aurantiaca, Newport \& Gray N. $56^{(1)}$. $H a b .:$ Rio de Janeiro.

20. Newportia (Scolopendrides) bicegoi Brölemann, 1903.

Newportia (Scolopentrides) bicegoi, Brölemann N. Hab. : (1) Manáos. 
21. Newportia (Scolopendrides) ernsti Pocock, 1891. Newportia Ernsti, Pocock N.o 91 c (1.2), 93 d ${ }^{(1.3)}$. Scolopendrides Ernsti, Pocock N. 95 h ${ }^{(2.3)}$.
Brölemann N. $98 \mathrm{e}^{(2)}$.

$H a b .:$ (1) Brésil.- (2) Carácas.-_(3) Antilles.

22. Newportia longitarsis (Newport), 1844.

Scolopocryptops longitarsis, Newport N. $44 \mathrm{c}^{(4)}$.

Newportia longitarsis,

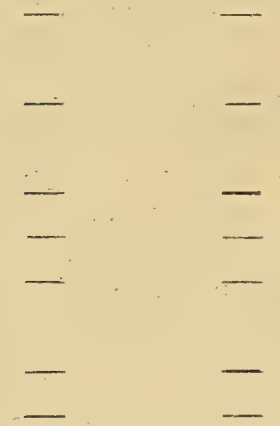

Gervais N. 47 a $^{(4)} 59^{(4)}$. Newport \& Gray N. $.56^{(4)}$.

Humb. \& Sauss. N. 69 b, $72^{(4)}$.

Kohlrausch N. $81^{(4)}$. Bollman N.0 88 c ${ }^{(4)}$. Pocock N. $93 \mathrm{~d}^{(4)}, 95$ $h^{(4)}$.

Silvestri N. ${ }^{0} 96 \mathrm{e}^{(3)}$. Brölemann N.o 98 e (2) $98 \mathrm{f}^{(2)}, 03$ a $^{(1)}$.

Hab. : (1) Manáos._- (2) Venezuela.-- (3) Colombie; Darien.-- (4) Antilles.

Mus. Paul.: Est. Amazonas, Manáos.

23. Newportia viridis (Gervais), 1847.

Scolopocryptops viridis, Gervais N. 47 a (1), $59{ }^{(1)}$. Newportia viridis, Newport \& Gray N. $56^{(1)}$. Hab: (1) Brésil.

12.e Genre: OTOCRYPTOPS Haase, 1887.

24. Otocryptops melanostoma (Newport), 1844.

Scolopocryptops melanostoma, Newport N. $44 \mathrm{c}^{(4)}$. Newport \& Gray N. $56^{(4)}$. 
Scolopocryptops melanosoma, Gervais N. 47 a ${ }^{(4)}$, $59^{(4)}$.

megacephalus, Kohlrausch N.o78(5), $81^{(5)}$.

$?$ - Karsch N. $84 b^{(6)}$. longiceps, Pocock N. $91 \mathrm{c}^{(1)}$. Otocryptops melanostoma, Poeock N.o $93 \mathrm{~d}$ (4), $95 \mathrm{~h}^{(1.3 .4)}$. Brölemann N. 98 $\mathrm{e}^{(2)}$

Hab.: (1) Brésil.— ${ }^{(2)}$ Venezuela._- ${ }^{(3)}$ Guatemala.(4) Ile St. Vicente.-( ${ }^{(5)}$ Ile Rosario.- ${ }^{(6)}$ Java?

Otocryptops punctatus $=$ Otocryptops sexspinosus.

25. Otocryptops sexspinosus (Say), 1821.

Cryptops sexspinosus,

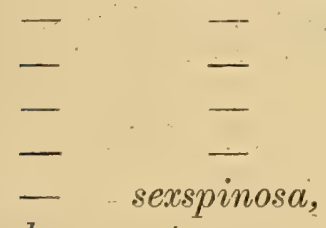

Say N.o $21^{(3)}, 22^{(3)}$.

Gervais N. 37 a $^{(3)}$.

Lucas N. $40 \mathrm{~b}$.

Newport N. 44 a.

C. Koch N. ${ }^{\circ} 47$.

Gray N. $44^{(3)}$.

Scolopocryptops sexspinosa, Newport N. 44 c ${ }^{(3)}$. Gervais N. ${ }^{\circ} 47^{\circ}$ a ${ }^{(3)}$, $59^{(3)}$.

Newport \& Gray N. $56^{(3)}$.

Wood N.o $63^{(3)}, 65$ a $^{(3)}$. Porat N. $76^{(1)}$.

sexspinosus Kohlrausch N.. $81^{(2)}$. Karsch N. 84 b.

Meinert N. 84 b (:), $85^{(i i)}$.

Underwood N. $87^{(3)}$. Mac-Neill N.: 87 a (3), $88^{(3)}$. 
Scolopocryptops sexspinosus, Bollman N. 88 a ${ }^{(3)}$, $88 \mathrm{~b}^{(3)}, 88 \mathrm{~d}^{(3)}, 88$ $\mathrm{f}^{(3)}, 88 \mathrm{~g}^{(3)}, 93^{(3)}$.

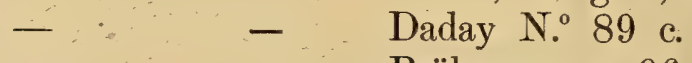

- B Bölemann n. $96 \mathrm{~h}^{(3)}$.

- spinicauda, Wood $\mathrm{N} .033^{(3)} 65 \mathrm{a}^{(3)}$.

? georgicus, Meinert N. $85^{\left({ }^{(3)}\right.}$ (sec. Bollman N. 93).

Scolopendropsis helvola, C. Koch N. $47^{(3)}, 63^{(3)}$. Otocryptops punctatus, Pocock n. $91 \mathrm{c}^{(4)}$. - sexspinosus, Pocock N.o 95 a ${ }^{(4)}$.

Non syn.: Scolopocryptops sexspinosus Kohlrausch, F vide Scolopocryptops miersi.

Hab.: (1) Brésil.- ${ }^{(2)}$ "America calidiore».-. ${ }^{(3)}$ E'tats Unis d'Amerique.- ${ }^{\left({ }^{(}\right)}$Chine.

13. Genre: OTOSTIGMUS Porat, 1876.

Otostigmus appendiculatus = Otostigmus seabricauda.

Otostigmus brasiliensis = Otostigmus scabricauda.

26. Otostigmus caudatus Brölemann, 1901.

Otostigmus caudatus, Brölemann N. ${ }^{\circ} 01 \mathrm{e}^{(1)}$. Hab.: Brésil; Itapetininga (S. P.), Belem (S. P.), Alto da Serra (S. P.).

Mus. Paul.: Est. S. Paulo, Itapetininga, Belen, Alto da Serra (Types).

27. Otostigmus goeldii Rrölemann, 1898.

Otostigmus Goeldii Brölemann N. $98 \mathrm{e}^{(2)}, 02 \mathrm{f}^{(1)}$. Hab.: (1) Pará- ${ }^{(2)}$ Venezuela. 
28. Otostigmus limbatus Meinert, 1884.

Otostigmus limbatus, Meinert N. 84 b ${ }^{(1.4)}$.

$\begin{array}{lll}- & - & \text { Karsch N. } 88 \mathrm{a} . \\ - & - & \text { Silvestri N.0 } 95 \mathrm{e}^{(3)}, 95 \mathrm{~d}^{(3)} . \\ - & \quad & \text { Brölemann N. } 4 \mathrm{e}^{(2)} .\end{array}$

Hab.: (1) Brésil._(2) Brésil ; Alto da Serra (S. P.).-

(3) Paraguay.- ${ }^{(4)}$ Buenos Aires.

Mus. Paul.: Brésil; Est. S. Paulo, Alto da Serra.

29. Otostigmus scabricauda (Humbert \& Saussure), 1870.

Branchiostoma scabricauda, Humb. \& Sauss. N. $70,72^{(1)}$

Branchiotrema sabricauda, Kohlrausch N. 78 ${ }^{(1.5)}, 81^{(1.5)}$.

Otostigmus appendiculatus, Porat N. $76^{(1)}$.

\begin{tabular}{|c|c|c|}
\hline- & brasiliensis, & Meinert N. $84 \mathrm{~b}^{(2.3)}$. \\
\hline & & Karsch N. 88 a. \\
\hline & scabricauda, & Pocock N. $90 \mathrm{~d}^{(6)}$ \\
\hline & & $\begin{array}{l}\text { Brölemann N.000 } \mathrm{g}^{(7)} \text {, } \\
\quad 01 \mathrm{e}^{(4)} \text {. }\end{array}$ \\
\hline
\end{tabular}

Hab.: (1) Brésil ; Rio de Janeiro.- ${ }^{(2)}$ Minas Geraes. ${ }^{(3)}$ Bahia.- ${ }^{(4)}$ Brésil ; Piquete (S. P.), Alto da Serra (S. P.).-- ${ }^{(5)}$ Popayan.- ${ }^{(6)}$ Andes de l'Equateur. - ${ }^{(7)}$ Guatemala.

Mus. Paul.: Brésil; Est. S. Paulo, Alto da Serra, Piquete.

30. Otostigmus tibialis Brölemann, 1901.

Otostigmus tibialis, Brölemann N. 01 e (1).

Hab.: (1) Brésil; Piquete (S. P.), Alto da Serra (S. P.). 
Mus. Paul.: Brésil; Est. S. Paulo, Santos, Alto da Serra, Piquete (Types).

Pithopus calcaratus = Scolopendropsis calcaratus. Pithopus inermipes = Scolopendropsis bahiensis. Rhoda Thayeri = Scolopendropsis thayeri.

14. Genre: RHYSIDA Wood, 1863.

31. Rhysida celeris (Humbert \& Saussure), 1870. Branchiostoma celer, Humb. \& Sauss. N. $70,72^{(8)}$. - ..- Kohlrausch N." $81^{(8)}$. - Meinert N.0 $84 \mathrm{~b}^{(2)}, 85^{(6.7)}$. - Underwood N. $87^{(8)}$.

Rhysida celeris, Pocok N. 93 d, 95 h ${ }^{(5 . \pi .8)}$. Silvestri $\mathrm{N} .95 \mathrm{c}^{(2)}, 95 \mathrm{~d}^{(1.3)} 97 \mathrm{~h}^{(4)}$. Hab.: (1) Manaos. - (2) Paraguay (Riacho del oro). - ${ }^{(3)}$ Argentine.-. ${ }^{(4)}$ Bolivia.- ${ }^{(5)}$ Venezuela (Bas Sarare).- - ${ }^{(6)}$ Nicaragua. - ${ }^{(7)}$ Antilles. - ${ }^{(8)}$ Carolina. Mus. Pauil.: Est. Amazonas, Manáos.

32. Rhysida longipes (Newport), 1844.

Branchiostoma longipes, Newport N. $44 \mathrm{c}$.

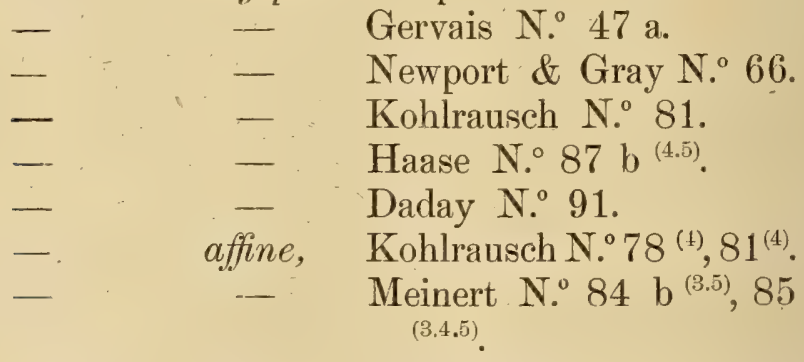

- gracile, Kohlrausch N. $78^{(5)}, 81^{(5)}$. obsoletum, Porat N. $76{ }^{(6)}$.

Rhysida longipes, Pocock N. $91 \mathrm{e}^{(5)}, 92$ b ${ }^{(5)}, 93$ $\mathrm{d}^{(3)}, 95 \mathrm{~h}^{(2.3)}, 01 \mathrm{e}^{(6)}$. 
Rhysida longipes, Silvestri N.o $94 \mathrm{e}^{(5)}$.

- $\quad$ - $\quad$ Attems N. $97 \mathrm{a}^{(5)}$.

Hab.: ${ }^{(1)}$ Bahia._- ${ }^{(2)}$ Mexique.-(3) Antilles.-. (1) Afrique.-- ${ }^{(5)}$ Asie (Malaisie).- - ${ }^{(6)}$ Australie.

Mus. Paul.: Est. Bahia.

15. Gevre: SCOLOPENDRA Linné 1758.

33. Scolopendra alternans Leach, 1814.

Scolopendra alternans, Leach N.. 14, 17.

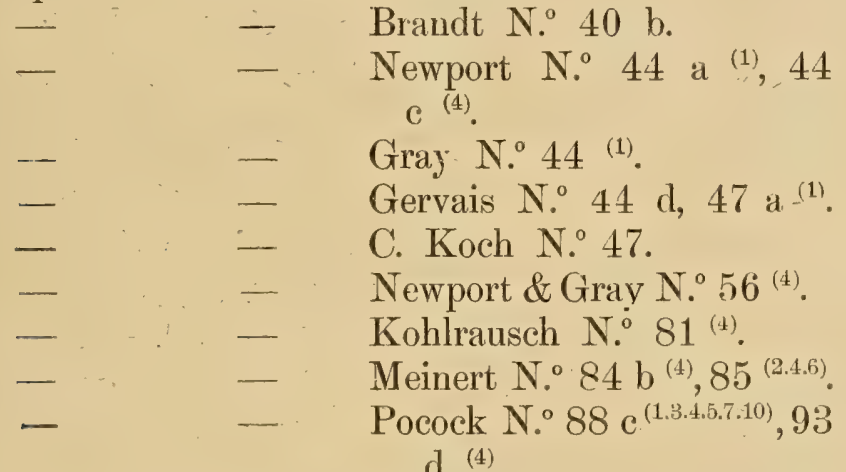

- complanata, Newport $\mathrm{N} .044 \mathrm{a}^{(5)}, 44 \mathrm{c}^{(5)}$. Gray N. $44^{(5)}$.

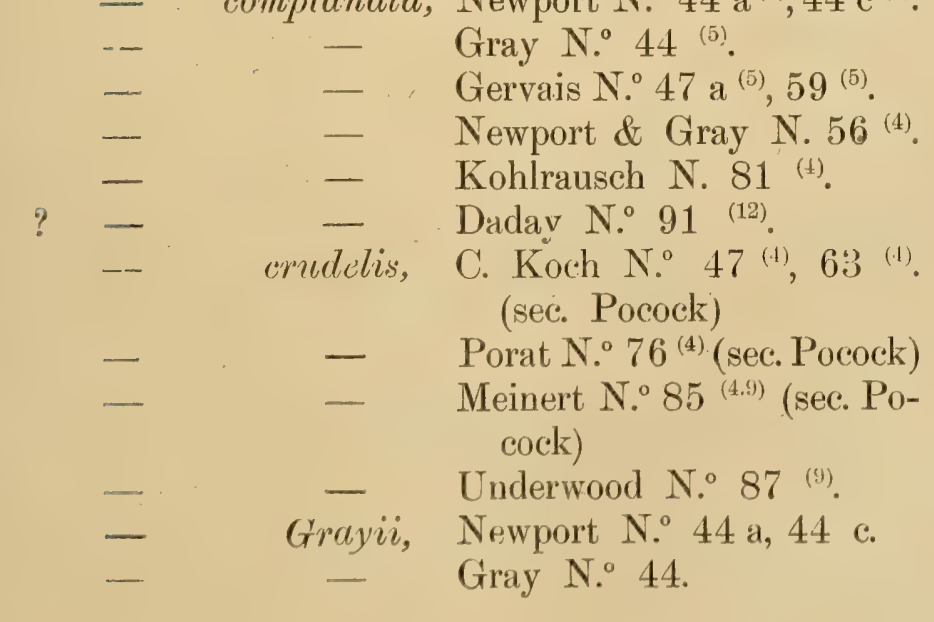


Scolopendra Grayii, Gervais N. 47 a.

Newport \& Gray N. 56.

Humbert N. $65^{(11)}$.

- inaequidens, Gervais N. $47 \mathrm{a}^{(9)}, 59^{(9)}$. (sec. Kohlrausch)

- incerta, Newport N. ${ }^{\circ} 44 \mathrm{c}$.

- - Gervais N. 47 a.

- - - Newport \& Gray N. 56.

- longipes, Wood N. $63^{(9)}, 65$ a $^{(9)}$. (sec. Pocock).

- multispinata, Newport N. $44 \mathrm{a}^{(5)}$.

\section{- $\quad$ - Gray N. $44^{(5)}$.}

- - Gervais N. $47 \mathrm{a}^{(5)}, 59^{(5)}$.

- multispinosa, Newport $\mathrm{N}^{\circ} 44 \mathrm{c}^{(5)}$.

- $\quad$ - Newport \& Gray N. $56^{(5)}$.

- Sagrae, Gervais N. 37 a ${ }^{(6)}, 47$ a ${ }^{(6)}$,

$$
59^{(6)} \text {. }
$$

- - Lucas N. $40 \mathrm{~b}^{(6)}$.

- $\quad$ Brandt N. $40 \mathrm{~b}^{(6)}, 41 \mathrm{~d}^{(6)}$.

- .. C. Koch N. 47.

- - R. de La Sagra N. $56^{(6)}$.

- testacea, Wood N. 61.

- torquata, Wood N. $61^{(8)}$.

Non syn.: Scolopendra complanata Latreille. inaequidens Wood.

Hab.: (1) Sud-A mérique.- - ${ }^{(2)}$ Brésil.- ${ }^{(3)}$ Colombie.(4) Antilles. -.- ${ }^{(5)}$ St. Christophe.- ${ }^{(6)}$ Cuba. -

${ }^{(7)}$ Dominica. - ${ }^{(8)}$ Sombrero Island.-- ${ }^{(9)}$ Etats Unis d'Amérique. - ${ }^{(10)}$ Sud-Afrique. - (11) Ceylon. - ${ }^{(12)}$ Caracas.--

34. Scolopendra angulata Newport, 1844.

Scolopendra angulata, Newport N. $44 \mathrm{a}^{(4)}, 44 \mathrm{c}^{(4)}$. Gray N: $44^{(4)}$.

Gervais $\mathrm{N}^{\circ} .47 \mathrm{a}^{(4)}, 59^{(4)}$. 
Scolopendra angulata, Newport \& Gray N. $56^{(t)}$.

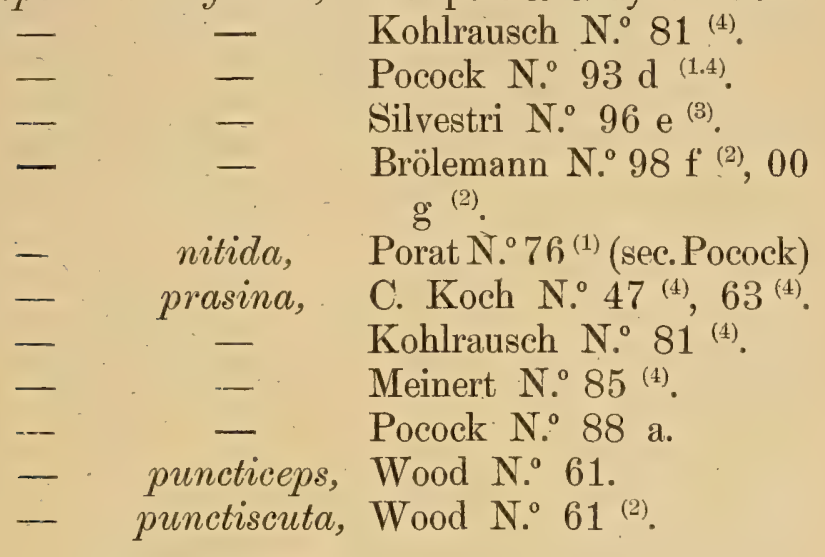

Hab.: (1) Brésil.- (2) Venezuela.- ${ }^{\left({ }^{(3)}\right.}$ Colombie.- ${ }^{( \pm)}$ Antilles.

35. Scolopendra annulipes Lucas, 1884.

Scolopendra annulipes, Lucas N. $84^{(1)}$.

Hab.: (1) Brésil.

Scolopendra audax =- Scolopendra subspinipes. Scolopendra aurantiipes = Cormocephalus aurantiipes.

Scolopendra (Scolopendropsis) bahiensis = Scolopendropsis bahiensis.

Scolopendra brandtiana - Scolopendra morsitans. Scolopendra bispinipes - Scolopendra subspinipes. Scolopendra complanata = Scolopendra alternans. Scolopendra cristata = Scolopendra viridicornis. Scolopendra crudelis = Scolopendra alternans. Scolopendra elonyata - Scolopendra subspinipes. Scolopendra epileptica :- Scolopendra gigantea. Scolopendra gervaisii - Scolopendra subspinipes. 
36. Scolopendra gigantea Linné, 1758.

Scolopendra epileptica, Wood N. $61^{(6)}$.

\begin{tabular}{|c|c|}
\hline igantea, & Linué N. ${ }^{\circ} 58$. \\
\hline - & Lueas N. $40 \mathrm{~b}^{(8)}$. \\
\hline 一. & Newport N. $44 \mathrm{c}^{(6)}$. \\
\hline - & Gray N. $0^{\circ} 44^{(1)}$. \\
\hline 一 & Gervais N. $37 \mathrm{a}^{(8)}, 47 \mathrm{a}^{(6)}$. \\
\hline- & Newport \& Gray N. ${ }^{0} 56^{(6)}$. \\
\hline 一 & Porat N. $76^{(4)}$. \\
\hline - & Daday N. $91^{(9)}$. \\
\hline - & Pocock N. $93 \mathrm{~d}^{(1.6)}$. \\
\hline gigas & Leach N. 14, 17. \\
\hline - & $\begin{array}{l}\text { Gervais N. } 37 \text { a, } 47 \text { a }^{(3)}, \\
59^{(3)} \text {. }\end{array}$ \\
\hline-- & Lucas N. $40 \mathrm{~b}$. \\
\hline- & Brandt N. $40 \mathrm{~b}, 41 \mathrm{~d}$. \\
\hline- & Newport $\mathrm{N}^{\circ} .44 \mathrm{a}^{(3)}, 44 \mathrm{c}^{(3)}$. \\
\hline - & Gray N..$^{\circ} 44^{(3)}$. \\
\hline - & Newport \& Grāy N. $56^{(3)}$. \\
\hline - & Kohlrausch No $81^{(1.6 .7)}$. \\
\hline - & Meinert N. $84 b^{(2.36)}, 85^{(2)}$. \\
\hline - & Pocock N. ${ }^{\circ} 88$ a, $95 \mathrm{~h}^{(\tilde{}}$. \\
\hline-- & Attems N. ${ }^{0} 00 \mathrm{~b}^{(4)}$. \\
\hline insignis, & Gervais \& Goudot N. ${ }^{\circ} 44^{(4)}$. \\
\hline - & Gervais N. $47 \mathrm{a}^{(4)}, 59^{(4)}$. \\
\hline - & Newport \& Gray N. $56^{(4)}$. \\
\hline- & Wood N. 63. \\
\hline prasinipe & Wood N. 61. \\
\hline
\end{tabular}

Non syn.: Scolopendra gigantea, C. Koch = Scol. subspinipes.

Hab.: (1) Sud-Amérique. - ${ }^{(2)}$ Brésil, Santarern, Obidos, Villa Bella.-_(3) Venezuela._- ${ }^{(4)}$ Colombie. - ${ }^{(5)}$ Honduras; Sud-Amérique; Jamaique. _- (6) Antilles._- ${ }^{(7)}$ Mexique.- ${ }^{(8)}$ America._- ${ }^{(9)}$ Trinidad; Maracaibo. 
Scolopendra grayii = Scolopendra alternans. Scolopendra herculeana = Scolopendra viridicornis. Scolopendra hopei = Scolopendra viridicornis. Scolopendra inaequidens = Scolopendra alternans. Scolcpendra incerta = Scolopendra alternans. Scolopendra insignis = Scolopendra gigantea.

37. Scolopendra leptodera Kohlrausch, 1878.

Scolopendra leptodera, Kohlrausch N. $78^{(1)}, 81^{(1)}$. $H a b .{ }^{(1)}$ Brésil.

Scolopendra longicornis = Scolopendra morsitans. Scolopendra longipes =- Scolopendra alternans. Scolopendra longipleura $=$ Scolopendra longispina.

38. Scolopendra longispina Meinert, 1885.

Scolopendra longipleura, Silvestri N.o $95 \mathrm{~d}^{(5)}, 97$. $b^{(5.6)}$.

- $\quad$ longispina, Meinert N. $84 \mathrm{~b}^{(3.4)}, 85^{(1)}$.

An syn.:-appendiculata, Daday N. 91.

$H a b$ : ${ }^{(1)}$ Brésil ; Maldonado.- ${ }^{(2)}$ Brésil; Belem (S. P.). - ${ }^{(3)}$ Montevideo.- ${ }^{(4)}$ Buenos Aires.-( ${ }^{(5)}$ Argentine; Tucuman.-- ${ }^{(6)}$ Bolivie.

Mus. Paul.: Est. Pará, Belem.

39. Scolopendra morsitans Linne, 1766.

Scolopendra Alfzelii, Porat $71^{(15)}$.

- algerina, Newport N. $44 \mathrm{c}^{(15)}$.

- angulipes, Newport N. $44 \mathrm{a}^{(15)}, 44$

- $\quad-\quad$ Gray N: $44^{(1 \overline{1})}$. 
Scolopendra angulipes, Gervais N. $47 \mathrm{a}^{(15)}$.

$\begin{array}{lll}- & - & \text { Newport \& Gray N. } 56^{(15)} . \\ - & \text { angusta, } & \text { Lucas N. } 39 \mathrm{a}^{(14)} \cdot \\ - & - & \text { Newport N. } 44 \mathrm{c}^{(14)} . \\ - & - & \text { Gray N. } 44^{(14)} \cdot \\ - & - & \text { Gervais N. } 47 \mathrm{a}^{(14)} . \\ - & - & \text { Newport \& Gray N. } 56^{(14)} .\end{array}$

- attenuata, Porat N. $71^{(15)}$.

- bilineata, (De Haan in litt., sec.) Brandt N: $40 \mathrm{~b}^{(18)}, 41$ $d^{(18)}$.

- $\quad$ Newport N. $44 \mathrm{e}^{(18)}$.

-. $\quad$ Gervais N. $47 \mathrm{a}^{(18)}$.

- $\quad$ - Newport \& Gray N. $56^{(18)}$.

- _- Tömösvary N. $85^{(18)}$.

- brachypoda, Peters N. $62^{(15)}$.

- Brandtiana, Gervais N. 37 a, 47 a

(2.7.10), $59^{(2.7 .10)}$.

- - Brandt N. 40 b, $41 \mathrm{~d}$.

- - L Lueas N. 40 c.

- $\quad$ - C. Koch N. 47.

- $\quad$ - Saussure N. $60^{(2.10)}$.

- californica, Humb. \& Sauss. N. 70, $72^{(12)}$.

- carinipes, Humb. \& Saúss. N. 70, $72^{(9)}$.

- chlorocephala, Porat N. $71^{(15)}$.

- cingulata, Gervais N. 74 a (excl. syn.) ${ }^{(15.18)}$.

- cognata, Porat N.o $71^{(15)}, 76^{(15)}$.

-- compressipes, Wood N. $63^{(19)}$.

- elegans, Gervais N.0 $47 \mathrm{a}^{(15)}$.

- erythrocephala, Brandt N. 40 b ${ }^{(18)}, 41$ $\mathrm{d}^{(18)}$.

- $\quad-\quad \underset{\mathrm{e}^{(18)} \text {. }}{\text { Newport N.0 } 44 \text { a }}{ }^{(18)}, 44$ 
Scolopendra erythrosephala, Gray N. 44.

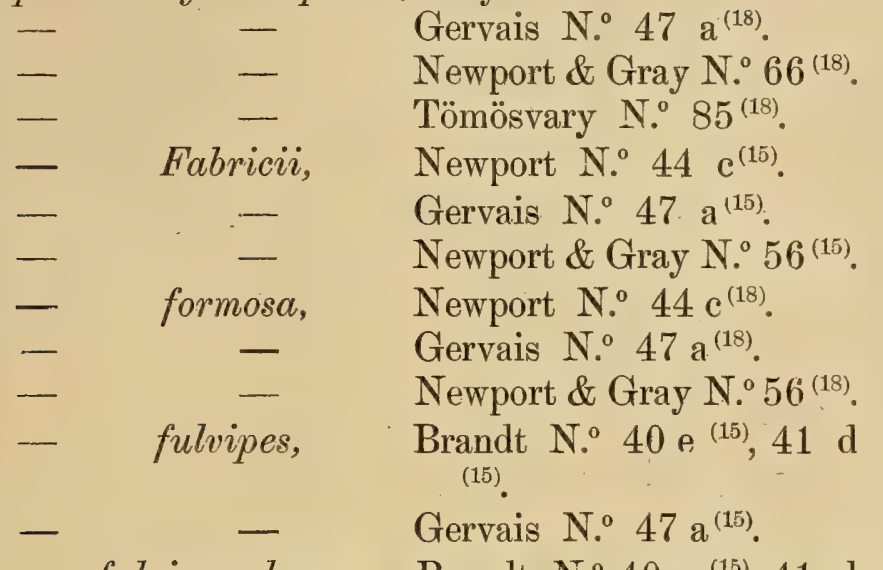

- fulvipes elegans, Brandt N.0 $40 \mathrm{e}^{(15)}, 41 \mathrm{~d}$ (15).

$\begin{array}{ccl}\text { - } & \text { gervaisiana, } & \text { C. Koch N. }:^{\circ} 63^{(15)} \\ \text { impressa, } & \text { Porat N.o 76 } \\ \text { - } & \text { infesta, } & \text { C. Koch N. } 47 ; 63 .\end{array}$

- intermedia, Porat N..$^{\circ} 1^{(15)}$.

- Leachii, Newport N. ${ }^{0} 44 \mathrm{a}^{(15)}, 44$

$\begin{array}{lll}- & - & \text { Gray N. }{ }^{\circ} 44^{(15)} \cdot \\ - & - & \text { Gervais N. } 47 \mathrm{a}^{(15)} \\ - & - & \text { Newport \& Gray N. } 56^{(15)} . \\ - & - & \text { Porat N.o 71 }\end{array}$

- longicornis, Newport N. 44 a $\stackrel{(19)}{9}, 44$ $\mathrm{e}^{(19)}$.

Gray N. $44^{(19)}$.

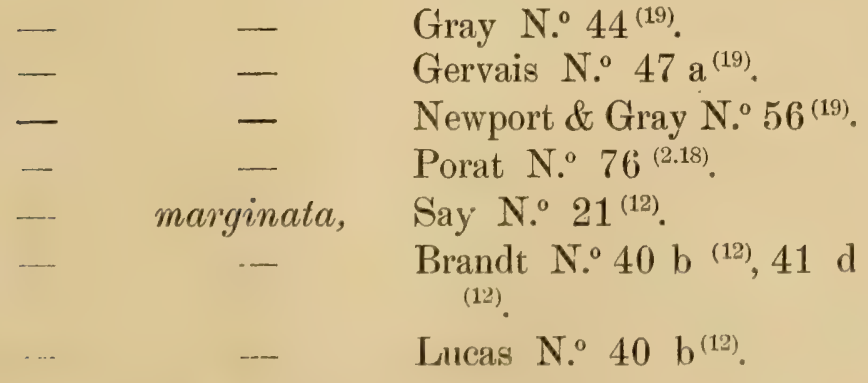


Scolopendra marginata, Gervais N.0 $37 \quad a^{(12)}, 47$ $\mathrm{a}^{(12)}, 59^{(12)}$.

- morsicans, exp., Gervais N.o $27 \mathrm{a}^{(15.18)}$. ex p., Lucas $\mathrm{N}^{\circ} 40 \mathrm{~b}^{(15.18)}$. - $\quad$ - Pocock N. $98 \mathrm{a}^{(19)}$.

- morsitans, Linné N. 66.

- $\quad$ Brandt N. d $^{(18)}$. 40 b ${ }^{(18)}, 41$

\begin{tabular}{|c|c|c|}
\hline - & - & $\begin{array}{c}\text { Newport N. } 44 \mathrm{a}^{(15)}, 44 \\
\mathrm{c}^{(10)} .\end{array}$ \\
\hline — & -- & Gray N. $0^{0} 44^{(15)}$. \\
\hline - & - & C. Koch N. ${ }^{\circ} 47^{(18)}$. \\
\hline- & - & Newport \& Gray N.. $56^{(10)}$. \\
\hline- & - & Wood N. ${ }^{\circ} 63^{(12)}, 65 \mathrm{a}^{(12)}$ \\
\hline - & - & $\underset{\text { 12.14.15.18.19). }}{\text { Kohlrausch }}$ N. ${ }^{\circ} 81^{(7.9 .10 .}$ \\
\hline- & - & Meinert N. 84 b, 85 . \\
\hline- & - & Tönösvary $\mathrm{N}^{\circ} \cdot 85^{(18)}$. \\
\hline- & - & Haase $N^{\circ} 87 b^{(10)}$ \\
\hline- & - & Underwood N. $87^{(12)}$ \\
\hline- & - & Daday N.o 89 c, 91, 93. \\
\hline- & - & $\begin{array}{l}\text { Pocock } \mathrm{N}^{0} 91 \mathrm{e}^{(18)}, 92 \mathrm{a} \\
\quad(15), 92 \mathrm{~b}^{(18)}, 93 \mathrm{a}^{(10)}, 93 \\
\mathrm{~d}^{(10)}, 94 \mathrm{~b}^{(18)}, 95 \mathrm{a}^{(18)}\end{array}$ \\
\hline- & - & $95 \mathrm{~h}^{(11)}, 96 \mathrm{e}^{(15)}$ \\
\hline & - & Bollman N.o $93^{(7)}$. \\
\hline$\ldots$ & - & $\begin{array}{l}\text { Porat N. } 94^{(10)} \\
\text { Silvestri }\end{array}$ \\
\hline & - & $\mathrm{b}^{(18)}, 95 \mathrm{c}^{(5)}, 95 \mathrm{~d}^{(5.6)}$ \\
\hline & - & $98 \mathrm{k}^{(15)}$. \\
\hline - & -- & $\underset{(18)}{\operatorname{Attems}} \mathbf{N} .^{\circ} 97 \mathrm{a}^{(18)}, 98 \mathrm{a}$ \\
\hline - & - & $\begin{array}{l}\text { Brölemann N.o } 01 \mathrm{e}^{(2)}, 02 \\
\mathrm{f}^{(3)}, ? 03 \mathrm{a}^{(4)}\end{array}$ \\
\hline
\end{tabular}

- mossambica, Peters N. $55^{(15)}, 62^{(15)}$. 
Scolopendra pella, picturata, - pilosella,

\section{- planipes,}

- platypoides,
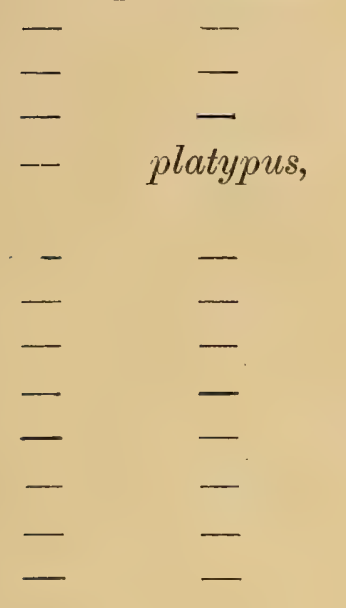

Wood N:o $61^{(7)}$.

Porat N. $71^{(15)}$.

Porat N.o $71^{(15)}$.

C. Koch N. $47^{(15)}, 63^{(15)}$.

Newport N. ${ }^{\circ} 44$ a, $44 \mathrm{e}^{(2)}$.

Gray N. 44.

Gervais N. 47 a ${ }^{(2)}, 59^{(2)}$. Newport \& Gray N. $56^{(2)}$. Brandt $\mathrm{N}^{\circ}{ }^{\circ} 40 \quad \mathrm{~b}^{(10)}, 41$ $d^{(10)}$.

Newport N. $44 \mathrm{a}^{(10)}$.

Gray $444^{(10 . ? 18)}$.

Gervais N. $47 \mathrm{a}^{(10)}, 59^{(10)}$.

R. de Ia Sagra N. ${ }^{0} 56^{(10)}$.

Humb. et Sauss. N. $72^{(8)}$.

Gerstäcker N. ${ }^{0} 73^{(15)}$.

Porat N. $76^{(1.10)}$.

Karsch N.o $81 \mathrm{~d}^{(19)}$.

Rainbow N. $97^{(19)}$.

- porphyrotaenia, Wood N.o $61{ }^{(18)}$.

- Richardsonii, Newport N.0 $44 \mathrm{e}^{(19)}$.

\begin{tabular}{|c|c|}
\hline - & Gray N. ${ }^{0} 44^{(19)}$. \\
\hline & Gervais N.0 $47 \mathrm{a}^{(19)}$. \\
\hline - & Newport \& Gray N. $56^{(19)}$ \\
\hline saltatoria, & Porat N. $71^{(15)}$. \\
\hline scopoliana, & C. Koch N. ${ }^{\circ} 41,47$. \\
\hline - & Gervals N. 48 a \\
\hline - & Karseh N.0 $81 a^{(15)}$. \\
\hline L- & Mattozo N: $81^{(10)}$. \\
\hline - & Pocock N. $91 \mathrm{~b}$. \\
\hline tigrina, & Newport N.o44 $\mathrm{e}^{(18)}$. \\
\hline- & Newport \& Gray $N^{0} 56^{(18)}$ \\
\hline tongana, & Gervais $\mathrm{N}^{0} 47 \mathrm{a}^{(18)}$. \\
\hline
\end{tabular}


Scolopendra tuberculidens, Newport N. ${ }^{\circ} 44$ a ${ }^{(18)}, 44$ $\mathrm{c}^{(18)}$.

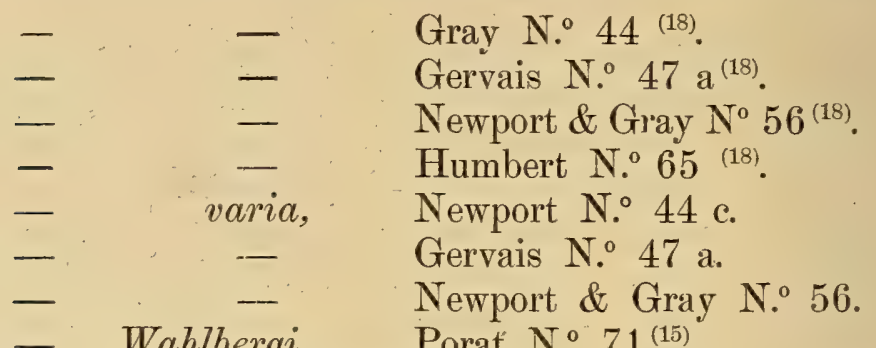

Eurylithobius slateri, Butler N. $76^{(13)}$.

Pocock N. 91 b.

? Heterostoma elegans, Newport N. $44 \mathrm{c}^{(15)}$.

- fulvipes, Newport N. 44 c.

Newport \& Gray N. 56.

An syn.: Scolopendra morsitans, Leach N. ${ }^{1} 7^{(18)}$.
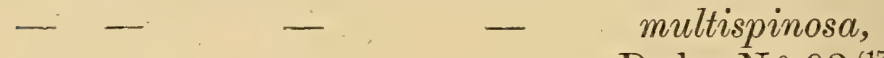

Daday N. $93^{(17)}$.

- - _ tigrina, Tömösvary N. 85

Non syn.: Scolopendra morsitans, C. Koch N. 63.

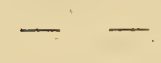

$-$

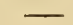

- cingulata,
N. ${ }^{2} 14$, Kohlrausch N. 81 . auct.

C. Koch 41, 47; Gervais, Lucas.

Hab.: (1) Sud-Amérique. - ${ }^{(2)}$ Brésil. - ${ }^{(3)}$ Pará. - ${ }^{(4)}$ Manáos._- ${ }^{(5)}$ Paraguay.- ${ }^{(6)}$ Argentine. - ${ }^{(7)}$ Guyanes.- ${ }^{(8)}$ Venezuela.- ${ }^{(9)}$ Colombie.- ${ }^{(10)}$ Antilles.-_(11) Mexique.-_ ${ }^{(12)}$ Etats Unis d'Amérique.-_(13) Ile Rodriguez.- ${ }^{(14)}$ Canaries. - ${ }^{(15)}$ Afrique.-- ${ }^{(16)}$ Europe. - ${ }^{(17)}$ Erivan, Caucase.-(18) Asie \& Malaisie.-(19) Australie.

Mus. Paul.: Est. Amazonas, Manáos (?) 
Scolopendra multispinata seu multispinosa $=$ Scolopendra alternans.

Scolopendra newportii = Scolıpendra subspinipes. Scolopendra nitida = Scolopendra angulata. Scolopendra ornata $=$ Scolopendra subspinipes. Scolopendra parva = Scolopendra viridis. Scolopendra placeae $=$ Scolopendra subspinipes. Scolopendra platypoides = Scolopendra morsitans. Scolopendra platypus = Scolopendra morsitans. Scolopendra prasina = Scolopendra angulata. Scolopendra prasinipes - Scolopendra gigantea. Scolopendra puncticeps = Scolopendra angulata. Scolopendra punctidens =- Scolopendra viridicornis. Scolopendra punctiscuta = Scolopendra angulata. Scolopendra punctiventris = Scolopendra viridis. Scolopendra sagrae $=$ Scolopendra alternans.

40. Scolopendra subspinipes Leach, 1814.

\section{Scolopendra}

\author{
atra,
} audax,
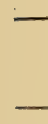

-

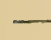

$-$

- aurantiipes,

- bispinipes,

- borbonica,

- bysina,

- cephalica,

-
Wood N. ${ }^{0} 61$.

Gervais N. 37 a ${ }^{(1)}, 47 \mathrm{a}^{(1)}$, $59^{(1.2)}$.

Brandt N. ${ }^{\circ} 40 \mathrm{~b}^{(? 10)}, 41 \mathrm{~d}$ ( ?10.

Lucas N. $40 \mathrm{~b}$.

Newport N. $44 \mathrm{c}$.

C. Koch N. 47 .

Newport \& Gray N. $56^{(2)}$.

Tömösvary N.o 85 (11).

Wood, N. $73^{(4)}, 65 \mathrm{a}^{(4)}$.

Blanchard N. 49 .

Wood N. 61 (4), $63^{(4)}$, 65 a ${ }^{(4)}$.

Wood N. 61 (7). cephalica gracilis, Wood N.: 
Scolopendra ceyionensis, Newport N. $44 \mathrm{c}^{(11)}$.

Gervais N. 47 a ${ }^{(11)}$.

Newport \& Gray N. ${ }^{0} 56^{(11)}$.

Humbert N: $65^{(11)}$.

- Childreni, Newport N. 44 a, 44 c.

Gray N. 44.

Gervais N: 47 a.

Newport \& Gray N. 56.

concolor, Newport N. $44 \mathrm{e}^{(11)}$.

Gervais N. $47 \mathrm{a}^{(11)}$.

Newport \& Gray N. $56^{(11)}$.

- damnosa, L. Koch N. $77^{(12)}$.

- De-Haani, Brandt N. 40 c (11) 41 $d^{(11)}$.

- - Newport N. $44 \mathrm{a} \mathrm{a}^{(11)}, 44$ c.

Gray N. $44^{(11)}$.

Gervais N. $47 \mathrm{a}^{(11)}$.

Newport \& Gray N. $56^{(11)}$.

exp., Kolhrausch N. ${ }^{\circ} 81^{(11)}$.

Meinert N. 84 b, $85^{(4.7 .11)}$.

Pocock N. 92 c (11).

Underwood N. $87^{(4)}$.

- dinodon, Wood N. $61^{(11)}, 63^{(11)}$.

- elongata, Porat N. $71^{(7)}, 76^{(1.2 .11 .13)}$.

- ferruginea, Brandt N. $40 \mathrm{~b}^{(7)}, 41 \mathrm{~d}^{(7)}$.

C. Koch N. $47^{(7)}, 63$.

- fissispina, L. Koch $65^{(11)}$.

- flava, Newport N. $44 \mathrm{c}^{(11)}$.

Gervais N: 47 a (11).

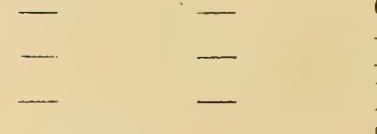

Newport \& Gray N. $56^{(11)}$.

Humbert N. $65^{(11)}$.

Tömösrary N. $85^{(11)}$.

- Gervaisii, Newport N. 44 c (1).

- gigantea, C. Koch N. ${ }^{\circ} 47^{(2)}, 63^{(2)}$.

- gracilipes, Wood 61 ${ }^{(11)}$.
-
Kohlrausch N. $81^{(11)}$. 
Scolopendra horrida,

- inermis,

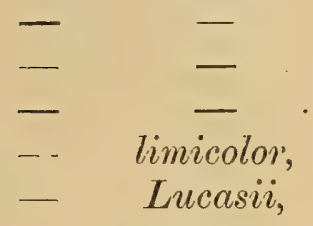

- lutea,

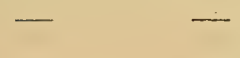

C. Koch N. $47^{(11)}, 63^{(11)}$ 。 Tömösvary N.o $85^{(11)}$.

Newport N. ${ }^{\circ} 44 \mathrm{c}^{(11)}$.

Gray N. ${ }^{0} 44^{\text {(11) }}$.

Gervais N. $47 \mathrm{a}^{(11)}$.

Newport \& Gray N. 56.

Wood N. $61^{(11)}$.

Gervais (Eydoux \& Souleyet) N.o $41{ }^{(9)}, 47 \mathrm{a}^{(11)}$.

Newport N. 44 c (2).

Gervais N. 47 a $^{(2)}, 59^{(2)}$.

Newport \& Gray N. ${ }^{5} 56^{(2)}$.

- macracanthus, Bollman N.o $89^{(5)}$.

- mactans, $\quad$ C. Koch N. $47^{(11)}, 63^{(11)}$.

- _ Tömösvary N. ${ }^{\circ} 85^{(11)}$.

- nesuphila, Wood N. $63^{(14)}$.

- Newportii, Gervais $\mathrm{N}^{\circ} 47 \mathrm{a}^{(1)}, 59^{(1)}$.

- $\quad$ - Newport \& Gray N.o $56^{(1)}$.

- ornata, Newport N. $44 \mathrm{c}$.

- $\quad$ - Gervais N. 47 a.

- $\quad$ C. Koch N. $47^{(1)}, 63^{(1)}$.

- - N Newport \& Gray N. $56^{\circ}$. parvidens, Wood N.o. 61 (i1). placeae, Newport N. $.^{0} 44 . \mathrm{c}^{(1)}$. Gray N. $44^{(1)}$.

Gervais N. 47 a (1), $59^{(1)}$. Newport \& Gray N. $56^{(1)}$. planiceps, Newport N. $44 \mathrm{c}^{(2)}$.

Gervais N. ${ }^{\circ} 47$ a ${ }^{(2)}, 59^{(2)}$.

Newport \& Gray N. $56^{(2)}$. plumbeolata, Wood N.o 61 (7). ? pulchra, C. Koch N. $47^{(2)} 63^{(2)}$. rarispina, Gervais N.0 47 a (7). sandwichiana, Gervais N. $47 \mathrm{a}^{(13)}$.

Wood N. $63^{(13)}$.

- septemspinosa, Brandt N.0 $40 \mathrm{~b}^{(11)}, 41 \mathrm{~d}^{(11)}$. 
Scolopendra septemspinosa, Newport N. $44 \mathrm{e}^{(11)}$.

\begin{tabular}{|c|c|c|}
\hline- & - & Gervais N. $0^{0} 47 \mathrm{a}^{(11)}$. \\
\hline- & - & Newport \& Gray N. $56^{(11)}$. \\
\hline & - & Wood N. $63^{\text {(11). }}$. \\
\hline & - & Karsch N. ${ }^{\circ} 80 \mathrm{~b}{ }^{(13)}$. \\
\hline & 一 & Tömösvary N. ${ }^{\circ} 85^{(11)}$. \\
\hline & sexspinosa, & Newport N. 44 a, 44 c. \\
\hline & - & Gray N: 44 \\
\hline & - & Gervais N. 47 a. \\
\hline & - & Newport \& Gray N. 56. \\
\hline & silhetensis, & Newport N.o $44 \mathrm{c}^{(11)}$. \\
\hline & - & Gervais $\mathrm{N}^{0} 47 \mathrm{a}^{(11)}$. \\
\hline & - & Newport \& Gray N.o $56^{(11)}$ \\
\hline & - & Porat N.o $76^{(11)}$ \\
\hline & subspinipes, & Leach N. ${ }^{\circ} 14,17$. \\
\hline & - & $\begin{array}{l}\text { Gervais }{ }^{\mathrm{N}} 0^{\circ} 37 \text { a, } 44 \mathrm{~d}^{(7)}, \\
\quad 47 \text { a } \\
\stackrel{(7)}{5}, 59 .\end{array}$ \\
\hline & - & $\begin{array}{c}\text { Brandt } \\
\mathrm{d}^{(11)} .\end{array}$ \\
\hline & - & Lucas N:o $40 \mathrm{~b}$. \\
\hline & - & Newport N. 44 a, 44 c. \\
\hline & - & Gray N. ${ }^{\circ} 44^{(11)}$ \\
\hline & - & C. Koch N ${ }^{\circ} 47$. \\
\hline- & - & Newport \& Gray N. 56. \\
\hline & - & Wood N. $63^{(2)}$. \\
\hline & - & Gerstäcker N.* $73^{(7)}$. \\
\hline & - & Kohlrausch N. ${ }^{\circ} 81{ }^{(1.2,11)}$ \\
\hline 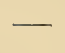 & - & Mattozo N. $81^{(7)}$. \\
\hline & - & Meinert N. ${ }^{\circ} 84$ b, 85 . \\
\hline & - & Haase $\mathrm{N}^{0} 87 \mathrm{~b}^{(11)}$. \\
\hline & - & Underwood N. $87^{(4)}$. \\
\hline & - & 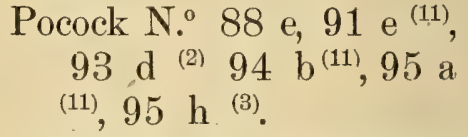 \\
\hline & - & Daday N.o 89 c, $91,93^{(14)}$. \\
\hline & -- & Bollman N.o 93 \\
\hline
\end{tabular}


Scolopendra subspinipes, Silvestri N.o 94 e (11), 95 $b^{(11)}$.

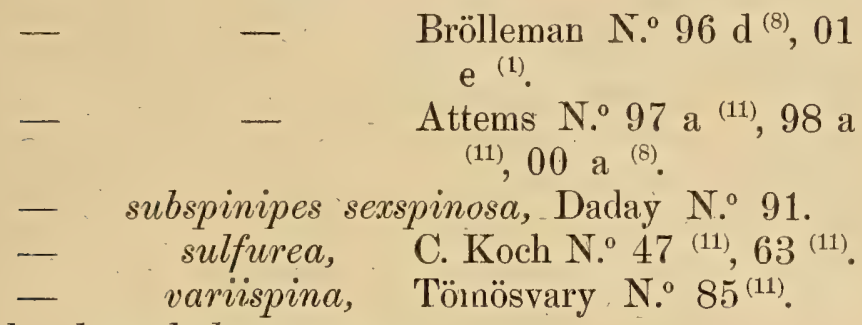

Rhombocephalus smaragdinus,

Butler N. $76^{(6)}$.

Non syn.: Scolopendra De-Haani ex p., Kohlrausch.

De-Haani histrionica, Meinert.

- flava, de Geer.

- gigantea, Linné et auct.

Hab.: (1) Brésil.-- ${ }^{(2)}$ Antilles et Amérique Ċentrale. - ${ }^{(3)}$ Mexique et Amérique Centrale.--(4) Etats Unis d'A mérique.- ${ }^{(5)}$ Côte du Pacifique.- - ${ }^{(6)} \mathrm{Ile}$ Rodriguez.- ${ }^{(7)}$ Afrique.- ${ }^{(8)}$ Séchelles.- ${ }^{(9)}$ Ile de France, de Bourbon et Mahé.-- ${ }^{(10)}$ Europe.(11) Asie plus Malaisie.-(12) Japon.- ${ }^{(13)}$ Australie. - ${ }^{(14)}$ Hawaï.

Mus. Paul.: Brésil.

Scolopendra testacea = Scolopendra alternans.

Scolopendra torquata = Scolopendra alternans.

Scolopendra variegata = Scolopendra viridicornis.

41. Scolopendra viridicornis Nerport, 1844.

S'colopendra cristata, Newport N.0 44 a ${ }^{(11)}, 44$ $\mathrm{c}^{(10)}$.

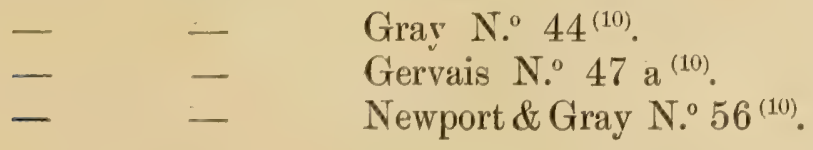


Scolopendra cristata, Porat N. $76^{(2)}$.

Kohlrausch. N. $81^{(2)}$.

- _ - Meinert N: $85^{(2.4)}$.

- herculeana, C. Koch N. $47^{(1)}, 63^{(3)}$,

- Hopei, Newport M. S. S. (sec. Gray

N. ${ }^{0} 56$ ).

- - Gray N. $44^{(2)}$.

- punctidens, Newport N. $44 \mathrm{a}^{(1)}, 44 \mathrm{e}^{(1)}$.

- - Gray N. $44^{(1)}$.

- _ Gervais N. $47 \mathrm{a}^{(1)}, 59^{(1)}$.

- - N Newport \& Gray N: $56^{(1)}$.

- - Kohlrausch N. ${ }^{\circ} 81^{(1)}$.

- variegata, Newport N. 44 a $^{(11)}, 44$ $\mathrm{c}^{(9)}$.

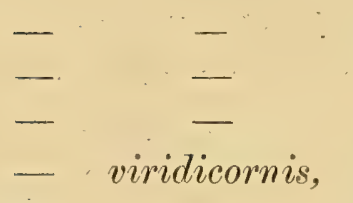

$-\quad-$

Gray N: $44^{(9)}$.

Newport \& Gray N. $56^{(9)}$.

Gervais N. 47 a $^{(11)}, 59^{(3)}$.

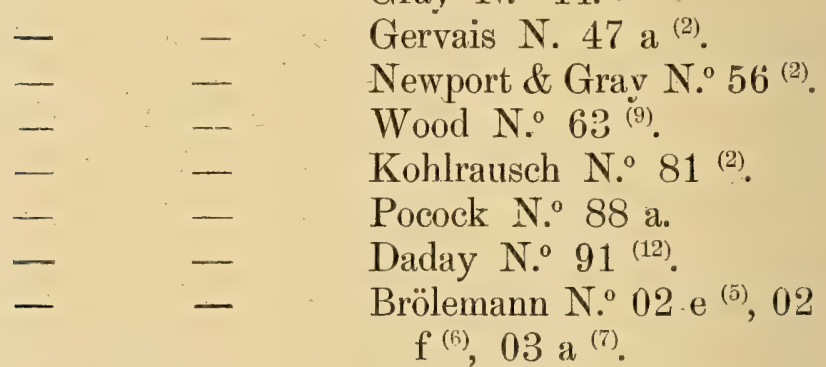

Hab.: (1) Sud-Amérique.-. (2) Brésil. - (") Région du Rio São Francisco._- (4) Brésil; Fleuve Amazone. - ${ }^{(5)}$ Brésil ; Sto. Antonio da Barra (Bahid), Pernambuco.-- ${ }^{(6)}$ Pará. — ( ${ }^{(7)}$ Manáos. - ${ }^{(8)}$ Paraguay. - ${ }^{(9)}$ Guyanes. — ${ }^{(10)}$ Chine?._ (11) Demerara._- (12) Caracas.

Mus. Paul.: Est. Bahia. 
42. Scolopendra viridis Say, 1821.

Scolopendra parva, Wood N. $61^{(5)}$.

- punctiventris, Newport N. $44 \mathrm{a}^{(5)}, 44 \mathrm{c}^{(5)}$.

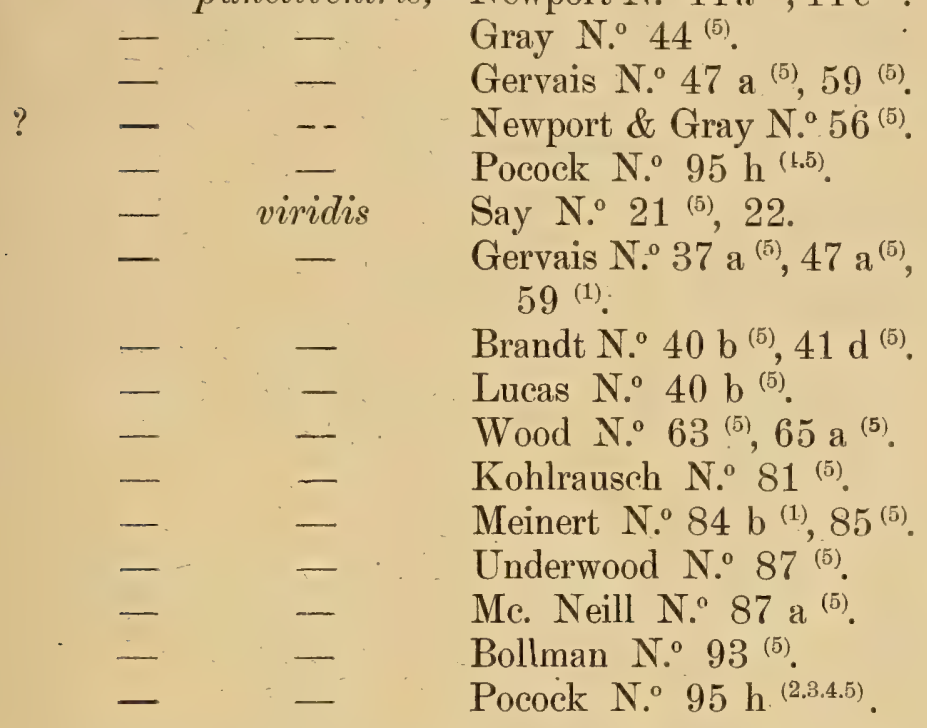

An syn.? Scolopendra atzeca, Saussure N. $60^{(4)} \cdot$

- $\quad$ - $\quad$ otomita, Saussure N.0 $60^{(t)} \cdot \mid \begin{gathered}\text { - } \quad \text { tolteca, Saussure N. }{ }^{\circ} 60^{(4)} \cdot\end{gathered}$

Hab.: ${ }^{(1)}$ Brésil._- ${ }^{(2)}$ Guatemala. - ${ }^{(3)}$ Costa-Rica.-- ${ }^{(4)}$.

Mexique.- ${ }^{(5)}$ Etats Unis d'Amérique.

Scolopendrides ernsti - Newportia (Scolopendrides) ernsti.

16." Genre: SCOLOPENDROPSIS Brandt, 1840.

4i. Scolonpendropsis bahiensis (Brandl), 1840.

Pithopus inermis, Pocock N. $91 \mathrm{~b}^{\text {(2) }}$ S'colopendra (Scolopendropsis) bahiensis, Brandt N. $40 \mathrm{e}^{(1)}, 41 \mathrm{~d}^{(1)}$. 
Scolopendropsis bahiensis, Newport N. $44 \mathrm{c}$.

- Gervais N. 47 a ${ }^{(1)}$.

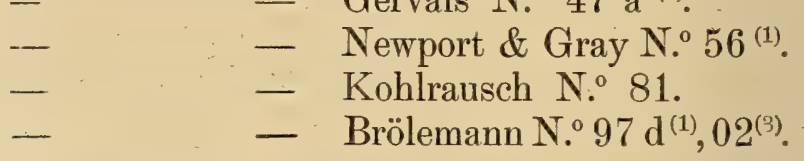

Hab.: ${ }^{(1)}$ Bahia._- ${ }^{(2)}$ Brésil ; Iguarassu. - ${ }^{(3)}$ Brésil;

Sto. Antonio da Barra (Bahia).

44. Scolopendropsis calcaratus $\left(P_{0 c o c k},\right) 1891$.

Pithopus calcaratus, Pocock N.o $91 \mathrm{~b}^{(1)}, 93 \mathrm{a}^{(1)}$. Scolopendropsis calcaratus, Brölemann N. $02 \mathrm{e}^{(2)}$. Hab.:- ${ }^{(1)}$ Bahia.- ${ }^{(2)}$ Brésil; Sto. Antonio da Barra (Bahia).

Scolopendropsis helvola $=$ Otocryptops sexpinosus.

45. Scolopendropsis thayeri (Meinert), 1885.

Rhoda Thayeri, Meinert N.o $85^{(1)}$.

$H a b .{ }^{(1)}$ Brésil ; Santarem.

17. Genre: SCOLOPOCRYTOPS Newport, 1844.

Scolopocryptops aurantiaca $=$ Newportia aurantiaca. Scolopncryptops georgicus=Otocryptops sexspinosus. Scolopocryptops longiceps = Otocryptops melanostoma.

Scolopocryptops longitarsis = Newportia longitarsis. Srolopocryptops megacephalus = Otocryptops melanostoma.

Scolopocryptops melanosoma seu melanostoma = Otocryptops melanostoma. 
46. Scolopocryptops miersi Newport, 1844.

Scolopocryptops Miersii, Newport N. $44 \mathrm{c}^{(1)}$.

$$
\begin{aligned}
& \text { - } \quad \text { - Gray N. }{ }^{\circ} 44^{(1)} \text {. } \\
& \text { - _ - Gervais N. } 47 \mathrm{a}^{(1)}, 59^{(1)} \text {. } \\
& \text { - } \quad \text { - Newport \& Gray N. } 56^{(1)} \\
& \text { - _ Karsch N. } 84 \mathrm{~b}^{(3.7)} \text {. } \\
& \text { - - Pocock } 93 \mathrm{~d}^{(6)} \text {. } \\
& \text { - - Silvestri N.095 } \mathrm{c}^{(4)} \text {. } \\
& \text { _- } \quad \text { Brölemann N. } 98 \mathrm{e}^{(5)}, 01 \\
& \mathrm{c}^{(2)}, 02 \mathrm{f}^{(3)} \text {. }
\end{aligned}
$$

Non syn.: Scolopocryptops Miersii, Meinert N. $84 \mathrm{~b}, 85$.

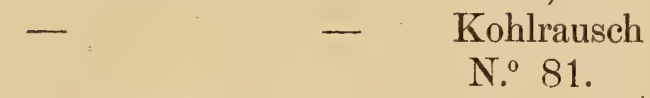

Hab.: (1) Brésil. - ${ }^{(2)}$ Brésil; São Lourenço (Rio Grande do Sul)._- ${ }^{(3)}$ Pará.-_ ${ }^{(4)}$ Argentine._ ${ }^{(5)}$ Venezuela.- - (6) Antilles.- (7) Californie.

Mus. Paul.: Est. R. Grande do. Sul, S. Lourenço. Scolopocryptops sexspinosa seu sexspinosus =Otocryptops sexspinosus.

Scolopocryptops spinicauda = Otocryptops sexspinosus.

Scolopocryptops viridis - Newportia viridis.

\section{ПNАMORРНА \\ 3. Fam. LITHOBIIDAE}

18." (ione: LITHOBIUS Leach, 1814.

47. I jithobius trilineatus $L$. Koch, 1862.

Lithobius trilineatus, L. Koch N.' $62^{(1)}$. 
(D'aprés Latzel, cette espèce tomberait en synonymie avec Lithobius forficatus, Linné.-Probablement importée).

Hab.: (1) Bahia.

\section{Fam. SCUTIGERIDAE}

\section{Genre: SCUTHGERA Lamarck, 1801.}

48. Scutigera nigrovittata Meinert, 1885.

Scutigera nigro-vittata, Meinert N. $85^{(4)}$.

$$
\begin{array}{ll}
- & \text { Pocock N.0 } 95 \mathrm{~h}^{(2.4)} \\
- & \text { Brölemann N.0 } 98 \mathrm{e}^{(2)}, 00 \\
& \mathrm{~g}^{(3)}, 02 \mathrm{e}^{(1)} .
\end{array}
$$

Hab.: (1) Brésil; Santo Antonio da Barra (Bahia).

- (2) Venezuela._( ${ }^{(3)}$ Colombie; Santa Marta.-

(4) Panamá.

\section{MYRIAPODA-DIPLOPODA}

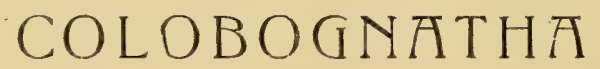

5. Fam. POLYZONIDAE

\section{0.e Genve: SIPHONOTUS Brandt, 1836.}

49. Siphonotus brasiliensis Brandt, 1831.

Siphonotus brasiliensis, Brandt N. 36,40 c (1), $41 \mathrm{~d}^{(1)}$.

Hab.: (1) Brésil. $\quad$ Gervais $\mathrm{N}^{\circ} 47 \mathrm{a}^{(1)}, 59^{(1)}$.




\section{JULOIDEA}

. 6. Fam. SPIROBOLIDAE

21. Genre: PARASPIROBOLUS Brölemann, 1801.

50. Paraspirobolus paulistus Brölemann, 1901.

Paraspirobolus paulistus, Brölemann N. 01 e (1). Hab.: (1) Brésil; Ilha de São Sebastião ( S. P.), Santos (S. P.).

Mus. Paul: Est. S. Paulo, Ilha de São Sebastião (Type), Santos.

22. (Y)enre: RHINOCRICUS Karsch, 1881.

51. Rhinocricus albidolimbatus (Porat), 1876 .

Spirobolus albido-limbatus, Porat N. ${ }^{\circ} 76^{(1)}$.

Rhinocricus albido-limbatus, Brölemann N.0 $02 \mathrm{e}^{(2)}$.

Hab.: (1) Olinda, Pernambuco.-- ${ }^{(2)}$ Brésil ; Pernambuco.

52. Rhinocricus asper Brölemam, 1901.

Rhinocricus asper, Brölemann N.o $01 \mathrm{e}^{(1)}, 02 \mathrm{e}^{(2)}$. Hab.: "1) Brésil ; Cubatão (S. P.), Poço-Grande (S.

P.), Alto da Serra (S. P.). - ${ }^{(2)}$ Brésil; Fazenda Nova Nicaragua (S. P.).

Mus. Paul.: Est. S. Paulo, Cubatão, Alto da Serra, Poço Grande (Types). 
53. Rhinocricus caudatus (Newport), 1844.

Iulus caudatus, $\quad$ Gervais N. 47 a $^{(1.4 .7)}, 59^{(4.7)}$. Rhinocricus caudatus, Brölemann N. $98 \mathrm{e}^{(5)}$. Spirobolus caudatus, Newport N. 44 b (4).
- _ - G Gray N. $44{ }^{(4)}$.
- $\quad$ Bollman N: $93^{(7)}$.

Spirobolus (Rhinocricus) laetus, Karsch N. 81 $g^{(3.5 .6 .8)}$. - Porat N. 88 b (1.5).

Hab. : (1) Brésil. - ${ }^{(2)}$ Guyane française.- ${ }^{(3)}$ Guyane anglaise.-_(4) Demerara._- ${ }^{(5)}$ Venezuela.- ${ }^{(6)} \mathrm{Co}-$ lombie._( ${ }^{(7)}$ S. Thomas. - ${ }^{(8)}$ Ternate.

54. Rhinocricus concinnus Brölemann, 1901.

Rhinocricus concinnus, Brölemann N. $01 \mathrm{e}^{(1)}$. Hab.: Brésil; Alto da-Serra (S. P.).

Mus. Paul.: Est. S. Paulo, Alto da Serra (Type).

55. Rhinocricus goeldii Brölemann, 1902.

Rhinocricus Goeldii, Brölemann N.o 02 f (1). Hab. : (1) Pará.

56. Rhinocricus insulsus Brölemann, 1902.

Rhinocricus insulsus, Brölemann N. ${ }^{\circ} 02 f^{(1)}$. Hab. : (1) Pará.

57. Rhinocricus limbatus Brölemann, 1901.

Rhinocricus limbatus, Brölemann N. $01 \mathrm{e}^{(1)}$. hab.: (1) Brésil; Poço-Grande (S. P.), Alto da Serra (S. P.).

Mus. Paul.: Est. S. Paulo, Alto da Serra (Type). 
58. Rhinocricus marginellus Silvestri, 1897.

Rhinocricus marginellus, Silvestri N. $97 \mathrm{e}^{(1)}$. (Peut être synonyme de $R h$. albidolimbatus Porat, 1876).

Hab. : (1) Pernambuco.

59. Rhinocricus moestus Brölemann, 1901.

Rhinocricus moestus, Brölemann N. $01 \mathrm{e}^{(1)}$. Hab.: (1) Brésil; Poço-Grande (S. P.), Alto da Serra (S. P.).

Mus. Paul.: Est. S. Paulo, Alto da Serra, Poço Grande (Types).

60. Rhinocricus monilicornis (Porat), 1876.

Rhinocricus monilicornis, Pocock N. ${ }^{\circ} 93 \mathrm{e}^{(3)}$. Spirobolus Heilprini, Bollman N.0 $93^{(4)}$. - moniticornis, Porat N..$^{0}$ (1). $^{(1)}$.

? - $\quad$ virescens, Daday N. $91^{(2)}$.

Hab. . (1) Brésil. _ ${ }^{(2)}$ Trinidad. -- ${ }^{(3)}$ Antilles. _- ${ }^{(4)}$ Bermudes.

61. Rhinocricus nattereri (Humbert \& Sanssure), 1870.

Rhinocricus Nattereri, Brölemann N. $01 \mathrm{e}^{(3)}$. Spirobolus Nattereri, Humb. \& Sauss. N. $70,72^{(1)}$. - Porat N. 88 b $^{(4)}$.

var.: varians Brölemann, 1901,

Rhinocricus Nattereri varians, Brölemann N.?01 $\mathrm{e}^{(3)}$. 
Hab.: (1) Brésil; Caiçara. - ${ }^{(2)}$ Bahia. - ${ }^{(3)}$ Brésil; São Sebastião (S. P.), Ilha de São Sebastião (S.P.), Raiz da Serra (S.P.), Paraná.--(4) Buenos-Aires. Mus. Paul.: Est. Bahia; v.varians, Est. S. Paulo, Raiz da Serra, S. Sebastião, Ilha de S. Sebastião; Est. Paraná.

62. Rhinocricus paraensis (Humbert \& Saussure), 1870.

Rhinocricus paraensis, Brölemann N.o $02 \mathrm{f}^{(1)} 03 \mathrm{a}^{(2)}$. Spirobolus paraensis, Humb. \& Sanss. N. $70,72^{(1)}$. Non syn.: Spirobolus paraensis, Pocock N. 88 c. Hab.: (1) Brésil ; Pará. - ${ }^{(2)}$ Manáus. Mus. Paul.: Est. Amazonas, Manáos.

63. Rhinocricus pugio Brölemann, 1901.

Rhinocricus pugio, Brölemann N.o $01 \mathrm{e}^{(1)}$. var:: ochrurus Brölemann, 1901.

Rhinocricus pugio ochrurus, Brölemann N. $01 \mathrm{e}^{(2)}$. Hab.: (1) Brésil ; Raiz da Serra (S.P.), Bahia.- (2)

Brésil; Alto da Serra (S.P.), Piquete (S.P.). Mus. Paul.: Est. Bahia; Est. S. Paulo, Raiz da Serra (Types); var. ochrurus, Est. S. Paulo, Alto da Serra (Type).

64. Rhinocricus sericiventris Brölemam, 1901 .

Rhinocricus sericiventris, Brölemann N ${ }^{0} 01 \mathrm{e}^{(1)}$. Hab.: (1) Brésil ; Cubatão (S. P.). Mus. Paul.: Est. S. Paulo, Cubatão (Type). 
65. Rhinocricus striatellus Silvestri, 1897.

Rhinocricus striatellus, Silvestri N..0 $97 \mathrm{e}^{(1)}$. Hab.: (1) Brésil ; Sta. Catharina.

66. Hhinocricus transver salis Brölemann,1902.

Rhinocricus transversalis, Brölemann N. $02 \mathrm{e}^{(1)}$. Hab.: (1) Brésil; Sto. Antonio da Barra (Bahia).

67. Rhinocricus zapotecus (Porat), 1876.

Rhinocricus zapotecus, Brölemann N.0 $02 \mathrm{e}^{(1)}$. Spirobolus zapotecus, Porat N. $76^{(1)}$. (? excl. syn.) An syn.: Iulus zapotecus, Saussure N. 60 (2). Hab.: (1) Pernambuco. - ${ }^{(2)}$ Mexique.

23. Genre: SPIROBOLELLUS Pocock, 1894.

68. Sipirobolellus cruentatus Brölemann, 1902.

Spirobolellus cruentatus, Brölemann N. $02 \mathrm{f}^{(1)}$. Hab.: (1) Pará.

24. Genre: SPIROBOLUS Brandt, 1833.

(Aucune des espèces qui figurent ici dans le genre Spirobolus n'ont été étudiées au point de vue des Pattes Copulatrices; il est donc impossible d'affirmer qu'il existe au Brésil de véritables spirobolus. Il est probable en tous cas que bon nombre des espéces ci-dessous appartiennent au genre Rlinocricu's. La plupart d'ailleurs ont été trop sommairement décrites pour qu'on puisse espérer les reconnaitre jámais.) 
Spirobolus albido-limbatus $=$ Rhinocricus albidolimbatus.

69. Spirobolus amazonicus (Giebel), 1870.

Iulus amazonicus, Giebel N.o $70^{(1)}$.

Hab.: (1) Brésil; les bords de l'Amazoue.

Spirobolus caudatus $=$ Rhinocricus caudatus.

70. Spirobolus dealbatus C. Koch, 1847.

Spirobolus dealbatus, C. Koch N. ${ }^{\circ} 47^{(1)}, 63^{(1)}$. Hab.: (1) Brésil.

71. Spirobolus dictyonotus Latiel, 1895.

Spirobolus dictyonotus, Latzel N.0 95 a ${ }^{(1)}$.

Hab.: Serres de Hambourg, probablement importé du Brésil.

Spirobolus dominicae =- Trigoniulus goësi.

72. Spirobolus festivus C. Koch, 1847.

Spirobolus festivus, C. Koch, N. $47^{(1)}$, $63^{(1)}$. Hab.: Brésil; Minas Geraes.

Spirobolus goësi $=$ Trigoniulus goësi.

73. Spirobolus grandis (Brandt), 1840.

Iulus (Spirobolus) grandis, Brandt N.o $40 \mathrm{~d}^{(1)}, 41 \mathrm{~d}^{(1)}$. - grandis, Gervais N.0 47 a ${ }^{(1)}, 59^{(1)}$.

? Spirobolus grandis, R. de la Sagra N. 56.

Hab.: (1) Brésil. 
74. Spirobolus griseus Nerport, 184.

Spirobolus griseus, Newport, teste Gray N.o $44^{(1)}$. Hab.: (1) Brésil.

Spirobolus heilprini = Rhinocricus monilicornis.

75. Spirobolus impressus Porat, 1876.

Spiroholus impressus, Porat N. $76^{(1)}$. ?

Hab.: (1) Pernambuco.

Spirobolus laetus = Rhinocricus caudatus.

76. Spirobolus laticaudatus Humbert \& Saussure, 1870.

Spirobolus laticaudatus, Humb. \& Sauss. N. 70 , $72^{(1)}$.

$H a b .{ }^{\text {(1) Brésil. }}$

77. Spirobolus macrourus Humbert \& Saussure, 1870.

Spirobolus macrourus, Humb. \& Sauss. N. ${ }^{\circ} 70$, $72^{(1)}$.

Non syn.: Spirobolus macrourus Pocock.

Hab.: (1) Brésil.

is. Spixobolus maximus (Linné), 1766.

Iulus maximus, Linné N. ${ }^{\circ} 66^{(1)}$.

\begin{tabular}{|c|c|c|}
\hline - & - & Fabricius N.o $81,87,93$. \\
\hline- & & Latreille N. 02. \\
\hline & - & Brandt N. 33. \\
\hline
\end{tabular}


Iulus (Spirobolus) maximus, Brandt N.o $40 \mathrm{~d}$ (1), $41 \mathrm{~d}^{(1)}$.

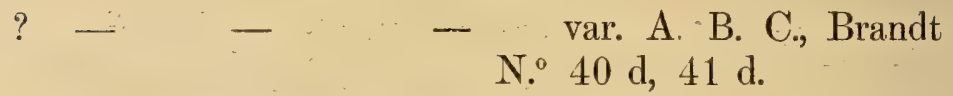

- maximus, Lucas N. 40 b ${ }^{(2)}$.

- _ Gervais N. $37 \mathrm{a}^{(2)}, 47 \mathrm{a}^{(1)}, 59^{(1)}$. Spirocyilisius maximus, C. Koch N. $47^{(2)}$. An syn.: Iulus apiculatus, Mikan N. 34. Spirobolus maximus, Tömösvary $\mathrm{N}^{\circ} .85^{(3)}$. Spirocyclistus apiculatus, C. Koch N. 47. maximus, C. Koch N. 63.

Hab.: (1) Brésil. - ${ }^{(2)}$ Amérique Méridionale. _. ${ }^{(3)}$ Borneo.

Spirobolus monilicornis = Rhinocricus monilicornis. Spirobolus nattereri = Rhinocricus nattereri.

79. Spirobolus nigroicans (Milian), 1834.

Iulus nigricans, Mikan N. 34.

- - Brandt N.040 $\mathrm{d}^{(1)}, 41 \mathrm{~d}^{(1)}$.

Spirobolus nigricans, C. Koch N. ${ }^{\circ} 47$.

Hab.: (1) Brésil.

80. Spirobolus obscurus C. Koch, $184 \%$.

Spirobolus obscurus, C. Koch N. 47, 63.

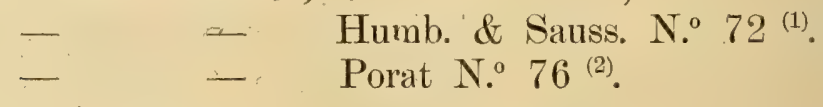

Hab.: (1) Brésil. _ (2) Rio de Janeiro.

81. Spirobolus obtusatus (Mikan), 1834.

Iulus obtusatus, Mikan N. 34.

Brandt N. $40 \mathrm{~d}^{(1)}, 41 \mathrm{~d}^{(1)}$. 
Iulus obtusatus, Gervais N.0 47 a ${ }^{(1)}, 59^{(1)}$. Spirobolus obtusatus, C. Koch N. 47.

Hab.: (1) Brésil.

82. Spirobolus olfersi (Brandt), 1833.

Iulus (Spirobolus) Olfersii, Brandt N.o $40 \mathrm{~d}$ (1), $41 \mathrm{~d}^{(1)}$.

- Olfersii, Gervais N. 47 a $^{(1)}, 59^{(1)}$. Spirobolus Olfersii, Brandt N. $33^{(1)}$.
- $\quad-\quad$ Gervais N: $37 \mathrm{a}^{(1)}$.
- - Lucas N. $40 \mathrm{~b}^{(1)}$.
- - C. Koch N.0 47.

Hab.: (1) Brésil.

Spirobolus paraensis =- Rhinocricus paraensis.

Spirobolus phranus ex p. = Trigoniulus goësi.

83. Spirobolus praelongus C. Koch, 1847.

Spirobolus praelongus, C. Koch N. $47^{(1)}, 63^{(1)}$. Spirobolus rugosus = Trigoniulus goesi.

84. Spirobolus striolatus (Gervais), 1847.

Iulus striolatus, Gervais N.. 47 a ${ }^{(1)}, 59^{(1)}$.

Hab.: (1) Amérique méridionale.

4.. Spirobolus univittatus Porat, 1876 .

Spirobolus univittatus, Porat N.: $76^{(1)}$ :

Hab.: (1) Brésil.

Spirobolus virescens - Rhinocricus monilicornis.

Spirobolus zapolecus =- Rhinocricus zapotecus. 
25. Genre: TRIGONIULUS Pocock, 1894.

86. Trigoniulus goësi (Porat), 1876.

Spirobolus Dominicae, Pocock N. ${ }^{\circ} 88 \mathrm{e}^{(3)}$.

\begin{tabular}{|c|c|c|}
\hline - & Goësi, & Porat N. ${ }^{0} 76^{(3.5}$ \\
\hline & & Pocock N. $92 \mathrm{~b}^{(5)}, 93 \mathrm{e}^{(5)}$. \\
\hline & - & Brölemann N. $96 \mathrm{~d}^{(4)}$. \\
\hline & phránus, & Pocock N. $0^{\circ} 88 \mathrm{e}^{(5)}$. \\
\hline & rugosus, & Voges N. $78 a^{(5)}$ \\
\hline & Goest & $\begin{array}{l}\text { Pocock } .93 \mathrm{e}^{(0)}, 94 \mathrm{~b}^{(5)} \text {. } \\
\text { Silvestri } 0^{\circ} 95 \mathrm{~b}^{(5)}\end{array}$ \\
\hline & - & Attems N. $.97 \mathrm{a}^{(5)}, 98 \mathrm{a}^{(5)}$. \\
\hline & - & $\begin{array}{l}\text { Brölemann } \mathrm{N}^{\circ}, 00 \mathrm{a}^{(1)}, 00 \\
\mathrm{~g}^{(3)}, 02 \mathrm{f}^{(1)}, 03 \mathrm{a}^{(2)} .\end{array}$ \\
\hline
\end{tabular}

An. syn.?: Spirobolus punctidives, Karsch N.ㄱ

$81 \mathrm{~g}^{(5)}$

punctiplenus, Karsch N.

$81 \mathrm{~g}{ }^{(5)}$
Sanctae-Luciae, Boll-

$\operatorname{man}$ N. $89^{(3)}$.

Non syn.: Spirobolus phranus Karsch.

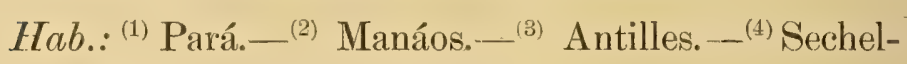
les. - ${ }^{(5)}$ Asie, plus Malaisie.

Mus. Paul.: Est. Amazonas, Manáos.

\section{Fam. SPIROSTREPTIDAE}

Alloporus longicornis:- Pseudonannolene longicornis.

26. Genre: ARCHISPIROSTREPTUS Silvestri, 1895.

(Ce genre v'ayant pas été établi sur des caractères sexuels, sa validité reste douteuse). 
Archispirostreptus lobulatus = Spirostreptus (Cladostreptus) lobulatus.

8i. Archispirostreptus punctiporus . Silvestri, 1897.

Archispirostreptus punctiporus, Silvestri N. ${ }^{\circ} 97 \mathrm{c}^{(1)}$. Hab.: (1) Brésil ; Rio Grande do Sul, Porto Alegre.

s8. Archispirostreptus sanctus Siliestri, 1897.

Archispirostreptus sanctus, Silvestri N.0 $97 \mathrm{e}^{(1)}$. Hab.: Brésil ; Sta. Catharina.

27. Genre: SPIROCYCLISTUS Brandt, 1833.

(Ce genre n'est admis par aucun auteur; il ne figure ici qu'à titre de document historique. L'espèce de Mikan n'est pas reconnaissable).

49. Spirocyclistus bicolor (Mikan), 1834.

Iulus bicolor, Mikan N. 34 .

- $\quad$ - Brandt N. ${ }^{\circ} 40 \mathrm{~d}^{(1)}, 41 \mathrm{~d}^{(1)}$.

-. - - Gervais N. $47 \mathrm{a}^{(1)}, 59^{(1)}$.

Spirocyclistus bicolor, C. Koch N. 47.

Hab.: (1) Brésil.

Spirocyclistus maximus =- Spirobolus maximus.

28." Girur: SPIROSTREPTUS Brandt, 1833.

90. Spirostreptus americanus (Brandl) 1 s t1).

Inlus americamus, Listser (Plumier) N.o 99.

Brandt N. $40 \mathrm{~d}^{(1)}, 41 \mathrm{~d}^{(1)}$.

Gervais N. 47 a ${ }^{(1)}, 59^{(1)}$. 
Non syn.: Iulus americanus, Palis. de Beauvois, Hab.: (1) Brésil.

Lucas, Gervais ete.

91. Spirostreptus (Cladostreptus) angustifrons Brölemann, 1901.

Spirostreptus (Cladostreptus) angustifrons, Brölemann N..0 $01 \mathrm{e}^{(1)}, 02 \mathrm{e}^{(2)}$. Hab.: (1) Brésil; Bahia. - (2) Brésil; St. Antonio da Barra (Bahia), Pernambuco. Mus. Paul.: Est. da Bahia (Type).

92. Spirostreptus bahiensis (Brandt), 1840. Iulus (Spirostreptus) bahiensis, Brandt, N. ${ }^{\circ} 40$ $\mathrm{d}^{(1)}, 41 \mathrm{~d}^{(1)}$.

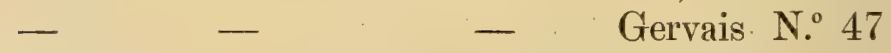

$H a b .:^{(1)}$ Bahia. $\mathrm{a}^{(1)}, 59^{(1)}$.

93. Spirostreptus biplicatus Karsch, 1881.

Spirostreptus biplicatus, Karsch N. $81 \mathrm{~g}{ }^{(1)}$. Hab.: (1) Rrésil.

94. Spirostreptus bipulvillatus (Gervais), 1847 .

Iulus bipulvillatus, Gervais N.? 47 a ${ }^{(1)}, 59{ }^{(1)}$. Hab.: (1) Brésil.

95. Spirostreptus caicarae Humbert \& Saussure, 1870.

Spirostreptus Caicarae, Humb.\& Sauss. N. $70,72^{(1)}$. Hab.: (1) Brésil; Caiçara. 
96. Spirostreptus cinctus Humbert of Saussure, 1870.

Spirostreptus cinctus, Humb. \& Sauss. N. $70,72^{(1)}$. Hab.: (1) Rio de Janeiro.

97. Spirostreptus (Scaphiostreptus) cluniculus Humbert \& Saussure, 1870.

Spirostreptus cluniculus, Humb. \& Sauss. N. 70, $72^{(1)}$.

- (Scaphiostreptus) cluniculus, Brölemann N.0 03 a $^{(2)}$.

Hab.: (1) Brésil ; Rio Negro.-- ${ }^{(2)}$ Manáos. Mus. Paul.: Est. Amazonas, Manáos.

98. Spirostreptus cultratus Humbert \& Saussure, 1870.

Spirostreptus cultratus, Humb. \& Sauss. N. 70. $72^{(1)}$

Hab.: (1) Brésil.

99. Spirostreptus (? Gymnostreptus) deserticola Brölemann, 1902.

Spirostreptus (? Gymnostreptus) deserticola, BröHab.: (1) Pernambuco. lemann N.0 $02 \mathrm{e}^{(1)}$.

100. Spirostreptus ( Cladostreptus) diptictus Brölemann, 1903.

sprirostreptus (cladostreptus) flavofasciatus, Brölemann $\mathrm{N}: 01 \mathrm{e}^{(1)}$. diptictus, Brölemann N. 03 a. 
Non syn.: Spirostreptus flavo-fasciatus Brandt. Hat.: Brésil; Piquete (S.P.).

Mus. Paul.: Est. S. Paulo, Piquete (Type de S. flavofasciatus Bröl.).

101. Spirostreptus (? Cladostreptus) dorsostriatus Brölemann, 1901.

Spirostreptus (? Cladostreptus) dorsostriatus, Brölemann N.0 $01 \mathrm{e}^{(1)}$.

Hab.: Brésil; Alto da Serra (S.P.).

Mus. Paul.: Est. S. Paulo, Alto da Serra (Type).

102. Spirostreptus excavatus harsch, 1881.

Spirostreptus excavatus, Karsch N. $81 \mathrm{~g}{ }^{(1)}$. Hab.: (1) Brésil.

103. Spirostreptus fartus Porat, 188.

Spivostreptus fartus, Porat N.0 88 b ${ }^{(1)}$. ITab.: (1) Brésil.

104. Spirostreptus festivus (Perty), 1853. Iulus festivus, Perty N.0 33. Gervais N. $37 \mathrm{a}^{(1)}, 47 \mathrm{a}^{(2)}, 59^{(2)}$. $-\quad-\quad$ Brandt N. $40 \mathrm{~d}^{(1)}, 41 \mathrm{~d}^{(1)}$. - - Lucas N. $40 \mathrm{~b}^{(2)}$.

Hab.: (1) Brésil._(2) Minas Geraes.

105. Spirostreptus (Cladostreptus) filum Brölemann, 1901.

Spirostreptus (Cladostreptus) filum, Brölemann N.o $01 \mathrm{e}^{(1)}$. 
Hab.: (1) Brésil; Alto da Serra (S. P.).

Mrus. Paul.: Est. S. Paulo, Alto da Serra (Type).

Spirostreptus (Cladostreptus) flavofasciatus - Spirostreptus (Cladostreptus) diptictus.

106. Spirostreptus (Scaphiostreptus) fuscipes Porat, 1888.

Spirostreptus fuscipes Porat N. 88 b ${ }^{(1)}$.

(Scaphiostreptus) fuscipes, Brölemann $\mathrm{N} .01 \mathrm{e}^{(2)}, 02 \mathrm{e}^{(2)}$.

Hab.: (1) Bahia, Pernambuco, Campos. - ${ }^{(2)}$ Bahia. Mus. Paul.: Est. Bahia, Bahia.

107. Spirostreptus (? Cladostreptus) gonnellei Brölemann, 1902.

Spirostreptus (? Cladosireptus) Gounellei Brölemann N. $02 \mathrm{e}^{(1)}$.

Hab.: Brésil; St. Antonio da Barra (Bahia).

108. Spirostreptus ( fiymuostreptus) iheringi Brijlemann, 1901.

Spirostreptus (Gymnostreptus) Iheringi, Brölemann N. $01 \mathrm{e}^{(1)}, 02 \mathrm{f}^{(2)}$.

Hab.: (1) Brésil ; Alto da Serra (S. P.), Morro Jaraguá (S. P.).- ${ }^{(2)}$ Theresopolis (R. de J.)

Mus. Praul.: Est. S. Paulo, Alto da Serra (Type), Morro Jaraguá.

109. Spirostreptus indus $1:$ Kowll, 1s/7.

Iulus affinis, Gervais, N. 47 ad ${ }^{(1)}$. 
Iuius (Spirostreptus) sebae, Hirandt, N.0 $40 \mathrm{~d}^{(1)}$, $41 \mathrm{~d}^{(1)}$.

- sebae, - Gervais N. $47 \mathrm{a}^{(1)}$. Spirostreptus indus, $\quad$ C. Koch N. $47^{(1)}$, $63^{(2)}$.

$\begin{array}{lll}- & \text { sebae, } & \text { Brandt N. } 33^{(1)} \\ - & - & \text { Gervais N. } 37 \mathrm{a}^{(1)} \\ - & - & \text { Lucas N. } 40.5 .\end{array}$

An sny.? Iulus indus, Linné N. $66^{(3)}$.

$$
\begin{aligned}
& \text { - - Fabricius N. }{ }^{7} 75,81,87 . \\
& \text { - - D De Geer N. } 78 . \\
& \text { - _ Olivier N. } 92 . \\
& \text { - - Gervais N. } 37 \mathrm{a}^{-(3)} \text {. } \\
& \text {-. - Lucas } \mathrm{N}^{\circ \circ} 40 \mathrm{~b}^{(3)} \text {. }
\end{aligned}
$$

Non syn.:- Iulus affinis, Lucas N. 49 c. - indus, Palis. de Beauvois.
Hab. :
(1) Patria ignota.-
${ }^{(2)}$ Brésil.-_( ${ }^{(3)}$ Inde?

110. Spirostreptus (Cladostreptus) interruptus Brölemann, 1901.

Spirostreptus (Cladostreptus) interruptus, Brölemann N.o $01 \mathrm{e}^{(1)}$.

Hab.: (1) Brésil ; Paraná.

Mus. Paul.: Est. Paraná (Type).

111. Spirostreptus (Cladostreptus) lobulatus (Attems), 1901.

Archispirostreptus lobulatus, Attems N.0 $01 \mathrm{~d}^{(1)}$. Hab.: (1) Blumenau (Sud-Brésil). 
112. Spirostreptus marus Karsch, 1881. Spirostrejtus marus, Karsch N. $81 \mathrm{~g}^{(1)}$. $H a b$. : (1) Serra Geral.

113. Spirostreptus mathematicus Krisch, 1881.

Spirostreptus mathematicus, Karsch N. $81 \mathrm{~g}{ }^{(1)}$. Hab. : Brésil.

114. Spirostreptus mellitus Karsch, 1881. Spirostreptus mellitus, Karsch N. $81 \mathrm{~g}{ }^{(1)}$. Hab.: (1) Serra Geral.

11\%. Spirostreptus microps Porat, 1876.

Spirostreptus microps, Porat N. $76^{(1)}, 88 b^{(2)}$. Hab.: (1) Corcovado et Pernambuco.- ${ }^{(2)}$ Brésil.

116. Spirostreptus occoecatus I'orat, 1888. Spirostreptus occoecatus, Porat N.o 88 b (1). Hab.: (1) Pedro de Cerra (Brésil ubi ?).

117. Spirostreptus ochrurus Porat, 1876.

Spirostreptus ochrurus, Porat, N. $76^{(1)}$. Hab.: (1) Rio de Janeiro, Corcovado.

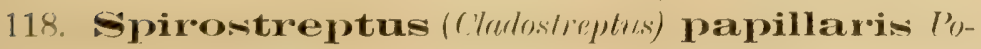
rat, 1876.

Spirostreplus papiliaris, Porat N. $76^{(1)}, 88$ b (1). 
An svn: Spirostreptus punctulatus, Karsch N. $81 \mathrm{~g}^{(2)}$.

Hab.: (1) Brésil._- ${ }^{(2)}$ Venezuela, San Fernando de Apure.

119. Spirostreptus (Cladostreptus) patruelis Porat, 1888.

Spirostreptus patruelis, Porat N. 88 b ${ }^{(1)}$.

(Cladostreptus) patruelis, Bröle-
mann N.o $01 \mathrm{e}^{(2)}$.

$H a b .:{ }^{(1)}$ Therezopolis (R. de Janeiro.- ${ }^{(2)}$ Brésil.

Piquete (S. P.).

Mus. Paul.: Est. S. Paulo, Piquete.

120, Spirostreptus (Gymnostreptus) perfidus Brölemann, 1901.

Spirostreptus (Gymnostreptus) perfidus, Brölemann N. $01 \mathrm{e}^{(1)}$.

Hab.: (1) Brésil; Alto da Serra (S. P.), Raiz da Serra (S. P.), Belém (S. P.); Paraná.

Mus. Paul.: Est. S. Paulo, Alto da Serra (Type).

Raiz da Serra; Est. Paraná.

121. Spirostreptus (Cladostreptus) perlucens Brölemann, 1901.

Spirostreptus (Cladostreptus) perlucens, Brölemann N." $01 \mathrm{e}^{(1)}$.

var. levior Brölemann, 1901.

Spirostreptus (Cladostreptus) perlucens levior, Brölemann $\mathrm{N}{ }^{\circ} 01 \mathrm{e}^{(1)}$. 
hab.: (2) Brésil ; Piquete (S. P.).

Mus. Paul.: Est. S. Paulo, Piquete (Type).

122. Spirostreptus (Alloporusi princeps Brölemann, 1901.

Spirostreptus (Alloporus) princeps, Brölemann N. $01 \mathrm{e}^{11}$.

Hab.: (1) Brésil ; Sta. Rita (S. P.).

Mus. Paul.: Est. S. Paulo, Santa Rita (Type).

123. Spirostreptus (? Scaphiostreptus) psendotuscipes Brölemann, 1902.

Spirostreptus (? Scaphiostreptus) pseudofuscipes, Brölemann $\mathrm{N}^{\circ} .02 \mathrm{f}^{(1)}$.

Hab. : (1) Pará.

124. Spirostreptus rotundanus Karsch, 1881. Spirostreptus rotundanus, Karsch N. $81 \mathrm{~g}{ }^{(1)}$. Hab.: (1) Rio de Janeiro.

Spirostreptus sebae $=$ Spirostreptus indus.

125. Spirostreptus (Clarlostreptus) sebastianus Brölemann. 1901.

Spirostreptus (Cladostreptus) sebastianus, Brölemann $\mathrm{N}^{\circ} 01 \cdot \mathrm{e}^{(1)}$.

Hab.: (1) Brésil; São Sebastião (S. P.), Ilha de São Sebastião (S. P.), Cubatão (S. P.).

Mus. Paul.: Est. S. Paulo, São Sebastião, (Type femelle), Tha de São Sebastião (Type mâle), Cubatão, 
126. Spirostreptus (Cladostreptus) semicinctus Brölemann, 1901.

Spirostreptus (Cladostreptus) semicinctus, Brölemann N.o 01 e ${ }^{(1)}$.

Hab.: Brésil: Poço Grande (S. P.).

Mus. Paul.: Esit. S. Paulo, Poço Grande (Type), Alto da Serra.

127. Spirostreptus (Alloporus) setiger Brölemann, 1901.

Spirostreptus (Alloporus) setiger, Brölemann N. $01 \mathrm{e}^{(1)}, 02 \mathrm{e}^{(2.4)}, 02 \mathrm{f}^{(3)}$.

Hab.: (1) Brésil ; Itapetininga (S. P.), Belém (S P.), Cerqueira Cezar (S. P.), Alto da Serra (S. P.); Bahia.-- ${ }^{(2)}$ Brésil ; Santo Antonio da Barra (Bahia). (3) Pará.- (4) Europe (importé).

Mus. Paul.: Est. S. Paulo, Belém, Itapetininga, Alto da Serra, Cerqueira Cezar (Types); Est. Paraná; Est. Bahia, Bahia; Est. A Amazonas, Manáos.

128. Spirostreptus strangulatus Humbert \& Saussure, 1870.

Spirostreptus strangulatus, Humb. \& Sauss. N. $70,72^{(1)}$.

Hab.: (1) Brésil ; Matto Grosso.

129. Spirostreptus (Gymnostreptus) subsericeus Rrölemann, 1901.

Spirostreptus (Gymnostreptus) subsericeus, Brölemann N. $01 \mathrm{e}^{(1)}$. 
var.: nitidior Brölemann, 1901.

Spirostreptus (Gymnostreptus) subsericeus nitidior, Brölemann N:01 $\mathrm{e}^{(2)}$.

Hab.: (1) Brésil ; Alto da Serra (S. P.), Cubatão (S. P.), Poço Grande (S. P.), Raiz da Serra (S. P.). - (2) Brésil; Cubatão (S. P.).

Mrus. Paul.: Est. S. Paulo, Alto da Serra, Raiz da Serra, Cubatão (Type).

130. Spirostreptus subuniplicatus (Brandt), 1840.

Iulus (Spirostreptus) subuniplicatus, Brandt N. ${ }^{\circ} 40$ $\mathrm{d}^{(1)}, 41 \mathrm{~d}^{(1)}$.

Iulus subuniplicatus, Gervais N. $47 \mathrm{a}^{(1)}, 59^{(1)}$.

Hab.: (1) Brésil.

131. Spixostreptus teres Humbert \& Sanssure, 1870.

Spirostreptus teres, Humb. \& Sauss. N. $70,72^{(1)}$. $H a b . \therefore$ (1) Brésil.

132. Spixostreptus torifer Porat, 1888.

Spirostreptus torifer, Porat N. 88 b ${ }^{(1)}$.

Hab.: (1) Therezopolis.

133. Spirostreptus trimarginatus (Grrais), 1847.

Iulus trimarginatus, Gervais No $47 a^{(1)}, 599^{(1)}$.

Hab.: (1) Brésil. 
134. Spirostreptus tristis Porat, 1888.

Spirostreptus tristis, Porat N. 88 b ${ }^{(1)}$.

Hab.: (1) Brésil; Pedro-Açu.

135. Spirostreptus (Gymnostreptus) ventralis Porat, 1876.

Spirostreptus ventralis, Porat N. $76^{(3)}, 88^{\text {b }}{ }^{(1)}$. Pocock N. $93 \mathrm{e}^{(3)}$. (Gymnostreptus) ventralis, Brölemann N. $01 \mathrm{e}^{(2)}$.

Hab.: (1) Brésil; Therezopolis.-(2) Brésil; Alto da Serra (S. P.), Cerqueira Cezar (S. P.), Os Perus (S. P.), Piquete (S. P.).- ${ }^{(3)}$ S. Thomas (Antilles). Mus. Paul.: Est. S. Paulo, Os Perús, Piquete, Alto da Serra, Cerqueira Cezar.

136. Spirostreptus volxemi Porat, 1888.

Spirostreptus, Volxemi, Porat N. 88 b ${ }^{(1)}$. Hab.: '(1) Therezopolis.

137. Spirostreptus ( G!mmostreptus) vulgatus Porat, 1888.

Spirostreptus vulgatus, Porat N. 88 b ${ }^{(1)}$. Hab. : (1) Brésil ; Therezopolis.

8. Fam PSEUDONANNOLENIDAE

29. Genre : PSEUDONANNOLENE Silvestri, 1895.

138. Pseudonannolene alegrensis Silvestri, 1897. 
Pseudonannolene alegrensis, Silvestri N.0 $97 \mathrm{c}^{(1)}$. Hab.: (1) Brésil ; Rio Grande do Sul, Porto Alegre.

139. Pseudonannolene callipyge Brölemann, 1901.

Pseudonannolene callypyge, Brölemann N.o $01 \mathrm{e}^{(1)}$. Hab.: (1) Brésil; Paraná. Mus. Paul.: Est. Paraná (Type).

140. Pseudonannolene exilis Brölemann, 1903. Pseudonannolene exilis, Brölemann N.․ 03 a (1). Hab.: (1) Manáos. Mus. Paul: Est. Amazonas, Manáos (Type).

141. Pseudonannolene longicornis (Porat), 1888.

Alloporus longicornis, Porat N.o $88 \mathrm{~b}^{(1)}$. var.: sebastianus Brölemann, 1901.

Pseudonannolene longicornis sebastianns, Brölemann $\mathrm{N}^{\circ} 01 \mathrm{e}^{(2)}$.

$H a b .:{ }^{(1)}$ Therezopolis. - ${ }^{(2)}$ Brésil ; São Sebastião

(S. P.), Santos (S. P.), Piquete (S. P.), Belém (S. P.), Alto da Serra (S. P.); Bahia.

Mus. Paul.: var. sebastianus Est. S. Paulo, São Sebastião (Type); Ilha de S. Sebastião, Santos, Alto da Serra, Cubatão, Belém, Piquete, Est. Bahia.

142. Pseudonannolene paulista brölemann, 1901.

Pseudonannolene paulista, Brölemann N.o $01 \mathrm{e}^{(1)}$,

$$
02 \mathrm{e}^{(2)}
$$


Hab.: (1) Brésil ; Cerqueira Cezar (S. P.). - ${ }^{(2)}$ Brésil; Fazenda Nova Nicaragua (S. P.).

Mus. Paul.: Est. S. Paulo, Cerqueira Cezar (Type).

143. Pseudonannolene tricolor Brólemanu, 1901.

Pseudonannolene tricolor, Brölemann N. $01 \mathrm{e}^{(1)}$. var.: gracilis Brölemann, 1901.

Pseudonannolene tricolor gracilis, Brölemann N. $01 \mathrm{e}^{(2)}$.

Hab.: (1) Brésil ; Itapetininga (S. P.), Alto da Serra (S. P.).- ${ }^{(2)}$ Brésil ; Piquete (S. P.).

Mus. Paul.: Est. S. Paulo, Itapetininga, (Type); var. gracilis, Est. S. Paulo, Piquete (Type).

\section{Fam. IULIDAE}

(On ne connait au Brésil aụcun représentant de cette famille. L'Iulus srassicornis de Mikan, le seul qui ne tombe pas en synonymie avec une espèce d'une autre famille, est trop sommairement décrit pour être reconnaissable et doit être considéré comme nomen nudum.)

30.e Genre: IULUS (Linné) Verhoeff,, (1746). (*)

Iulus abbreviatus = Polydesmus abbreviatus. Iulus affinis = Spirostreptus indus.

Iulus amazonicus = Spirobolus amazonicus.

Iulus americanus = Spirostreptus americanus. Iulus apiculatus = Spirobolus maximus.

(*) Cf. Obs. de la Redaction, p. IV. 


\section{POLYDESMOIDEA \\ 10. Fam. POLYDESMIDAE}

Catharodesmus alegrensis = Leptodesmus alegrensis.

Catharodesmus lartiguei = Leptodesmus lartiguei.

31. Genre: CRYPTODESMUS Peters, 1864.

145. Cryptodesmus olfersi (Brandt), 1839.

Polydesmus Olfersii, Brandt N. $39 \mathrm{a}^{(1)}, 41 \mathrm{~d}^{(1)}$.

Gervais N. 47 a $^{(1)}, 59^{(1)}$.

- (Rachophorus) olfersii, Peters N. $64^{(1)}$.

- (Cryptodesmus) - Peters N.064 suppl.

Cryptodesmus olfersii, Attems N. $99 \mathrm{~b}{ }^{(1)}$.

Hab.: (1) Brésil.

146. Oryptodesmus pileolus Bröleinam, 1903.

Cryptodesmus pileolus, Brölemann N. 03 a ${ }^{(1)}$.

Hab.: (1) Brésil; Poço Grande (S. P.).

Mus. Paul.: Est. S. Paulo, Poço Grande (Type).

147. Cryptodesmus politulus Brölemann, 1903.

Cryptodesmus politulus, Brölemann N.0 $03 \mathrm{a}^{(1)}$.

Hab.: (1) Brésil; Poço Grande (S. P.).

Mus. Paul.: Est. S. Paulo, Poço Grande (Tvpe).

148. Cryptodesmus pusillus Attems, 1899.

Cryptodesmus pusillus, Attems N. 99 b (1).

Hab.: Santa Cathariua (Brésil). 


\section{2.e Genre: CRYPTURODESMUS Silvestri, 1897.}

149. Crypturodesmus targionii Silvestri, 1897.

C.ypturodesmus Targionii, Silvestri N. 97 a $^{(1)}$. Attems N. 99 b.

Hah.: (1) Brésil (indiqué par erreur du Mexique par Silvestri N. 97 a).

33. Genre: EURYDESMUS Saussure, $186^{\circ} 0$.

150. Eurydesmus angulatus Sansmire, 1960.

Eurydesmus angulatus, Saussure N. $60^{(1)}$.

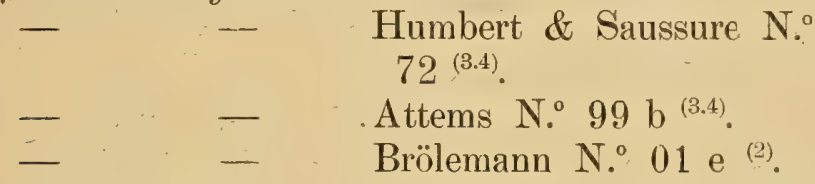

$H a b$ : (1) Le Brésil, selon toute probabilité. -

Brésil; Alto da Serra (S. P.).-- ${ }^{(3)}$ Rio de Janeiro. - (4) Argentine.

Mus. Paul.: Brésil; Est. S. Paulo, Alto da Serra.

34. Genve: EURYURUS $C$. Koch, 1847.

151. Euryurus elongatus Brölemann, $190 \%$.

Euryurus elongatus, Brölemann N. 03 a ${ }^{(1)}$. Hab.: (1) Manáos.

152. Euryuxus octocentrus Brilfmann, 19013.

Euryurus elongatus, Brölemann N. (0) at (1). Hab.: (1) Manáos. 
35. Genre: FONIARIA Gray, 1832.

153. Fontaria carnea Newport, 1844.

Fontaria carnea, Newport, teste Gray N." $44^{(1)}$. Hab.: (1) Brésil.

154. Fontaria dubia Newport, 1844.

Fontaria dubia, Newport, teste Gray N. $44^{(1)}$. Hab.: (1) Brésil.

Fontaria gracilis = Orthomorpha gracilis.

155. Fontaria zebrata (Gervais), 1836.

Polydesmus zebratus sen zonatus, Gervais N. $36 \mathrm{~b}^{(1)}$,

\begin{tabular}{|c|c|c|}
\hline- & - & Gervais $N^{\circ} 37 \mathrm{a}^{(1)}$. \\
\hline & & $\begin{array}{l}\text { seu zonatus, Brandt } \mathrm{N} \\
39 \mathrm{a}^{(1)}, 41 \mathrm{~d}^{(1)}\end{array}$ \\
\hline & (Fontaria) & $\begin{array}{l}\text { Lucas N. } 40 \mathrm{~b}^{(1)} \\
\text { zebratus, Gervais N. }\end{array}$ \\
\hline
\end{tabular}

Hab.: Jrésil.

36. Genre: KATANTODESMUS Attems, 1899.

156. Katantodesmus auriculatus Attems, 1899 Katantodesmus auriculatus, Attems N.0 99 b (1). Hab.: (1) Brésil ; Santa Catharina.

157. Katantodesmus scapulatus Attems, 1899. Katantodesmus scapulatus, Attems N.o 99 b ${ }^{(1)}$. Hab.: (1) Brésil; Santa Catharina. 
37. Genre: LEPTODESMUS Saussure, 1859.

158. Leptodesmus (Rachidomorpha) aduncus (Humbert of Saussure), 1872.

Leptodesmus (Rachidomorpha) aduncus, Attems N.0 $01 \mathrm{e}^{(1)}$.

Polydesmus uncinatus, Humb. \& Sauss. N. 69 b ${ }^{(1)}$. -- (Rachidomorpha) [uncinatus] aduncus, Humb. \& Sauss. N. $72^{(2)}$. Rachidomorpha aduncus [uncinatus], Attems N. $98 \mathrm{~b}^{(2)}$.

Hab.: (1) Brésil ; Espirito Santo.- ${ }^{(2)}$ Mexique.

159. Leptodesmus alegrensis (Silvestri), 1897. Catharodesmus alegrensis, Silvestri N.0 $97 \mathrm{e}^{(1)}$. Hab. (1) Brésil; Rio Grande do Sul, Porto Alegre.

160. Leptodesmus appendiculatus Attems, 1901.

Leptodesmus appendiculatus, Attems N. $01 \mathrm{c}^{(1)}$. Hab.: (1) Brésil ; Petropılis.

161. Leptodesmus bahiensis Brölemann, 1902. Leptodesmus bahiensis, Brölemann N.0 $02 \mathrm{e}^{(1)}$. Hab.: (1) Brésil; Santo Antonio da Barra (Bahia)

162. Leptodesmus bicolor Brölemann, 1901.

(? Rachidomorpha) bicolor, Brölemann N.0 $01 \mathrm{e}^{(1)}$. Hab.: (1) Brésil ; Piquete (S. P.). Mus. Paul.: Esst. S. Panlo, Piquete (Type). 
163. Ieptodesmus biconicus Attem, 1898.

Leptodesmus biconicus, Attems N.0 98 b ${ }^{(1)}, 01 \mathrm{e}^{(2)}$. Hab.: (1) Rio de Janeiro, Corcovado.-- ${ }^{(2)}$ Petropolis.

164. I eptodesmus bidens Brölemann, 1901.

Leptodesmus bidens Brölemann N.0 $01 \mathrm{e}^{(1)}$. Hab.: - ${ }^{(1)}$ Brésil ; Piquete (S. P.).

Mus. Paul.: Est. S. Paulo, Piquete (Type).

165. ( ? Leptodesmus) brasiliae Brïlemmn, 1901.

Rachidomorpha Brasiliae, Brölemann N.o $01 \mathrm{e}^{(1)}$. D'après les plus récents travaux d'Attems, cette espèce diffère des Rachidomorpha typiques dans la forme des Pattes Copulatrices; il y aura lieu de lui fixer sa place; provisoirement nous la laissons dans le genre Leptodesmus.

ITab.: (1) Brésil; Piquete (S. P.).

Mus. Paul.: Est. S. Paulo, Piquete (Type).

166. Leptodesmus carinovatus Attems, 1898. Leptodesmus carinovatus, Attems N. 98 b (1). Brölemann N.0 03 a ${ }^{(1)}$.

Hab.: (1) Manáos.

Mus. Paul.: Est. Amazonas, Manáos.

167. Leptodesmus carminatus Brölemann, 1902.

Leptodesmus carminatus, Brölemann N. $02 \mathrm{e}^{11)}$. Hab.: (1) Brésil; Santo Antonio da Barra (Bahia). 
168. Leptodesmus carneus (Saussure), 1859.

Leptodesmus carneus, Attems $\mathrm{N}^{\circ} 98 \mathrm{~b} \mathrm{~b}^{(2.8)}$. Polydesmus carneus, Gervais N. $59^{(1)}$. - (Leptodesmus) carneus, Saussure N. $59^{(1)}, 60^{(3)}$. Polydesmus (Oxiurus) carneus, Humb. \& Sauss. $\mathrm{N} .72^{(2)}$. Hab.: (1) Brésil.- ${ }^{(2)}$ Riı) de Janeiro.-- ${ }^{(3)}$ Bahia.

169. Leptodesmus centropus Attems, 1898.

Leptodesmus centropus, Attems N. $98 \mathrm{~b}^{(1)}$. $H a b .:$ (1) Sta. Catharina.

170. Leptodesmus cinerascens ( $C$. Koch $), 1847$. Oxyurus cinerascens, C. Koch N.0 $47^{(1)}, 63^{(1)}$. Hab.: (1) Brésil.

171. Leptodesmus clavaria Brölenann, 1902. Ledtodesmus clavaria, Brölemann N.002 $\mathrm{e}^{(1)}$. Hab.: ${ }^{(1)}$ Brésil ; Fazenda Nova Nicaragua (S. P.).

172. Leptodesmus cognatus Brölemann, 1901. Leptodesmus cognatus, Brölemann N.0 $01 \mathrm{e}^{(1)}$. Hab.: (1) Brésil; Alto da Serra (S. P.). Mus. Paul.: Est. de S. Paulo, Alto da Serra (Type).

173. Leptodesmus conspersus (Perty), 18:3\%3. Polydesmus conspersus, Perty N. 33. Gervais N.o $37 \mathrm{a}^{(1)}, 4 \mathrm{r}^{(2)}$, $59^{(1)}$. 
Polydesmus conspersus, Brandt N. ${ }^{\circ} 39 \mathrm{a}^{(2)}, 41 \mathrm{~d}^{(2)}$. Lucas N. $40 \mathrm{~b}^{(2)}$.

Rhacophorus conspersus, C. Koch N. 47, $63^{(1)}$.

Hab.: (1) Brésil.- ${ }^{(2)}$ Minas Geraes.

174. Leptodesmus corcovadis Brölcmann, 1902.

Leptodesmus corcovadis, Brölemann N. $02 \mathrm{e}^{(1)}$. Hab.: (1) Brésil; Mt. Corcovado, R. de Janeiro.

175. Leptodesmus (Rachidomorpha) corniger Brölemann, 1903.

Leptodesmus (Rachidomorpha) corniger, BröleHab.: (1) Brésil ; Rio-Grande (S. P.).

176. Leptodesmus cylindricus Brölemann 1901. Leptodesmus cylindricus, Brölemann $\mathrm{N}^{0} 01 \mathrm{e}^{(1)}$. Hab.: ${ }^{(1)}$ Brésil ; Piquete (S. P.).

177. Leptodesmus decipiens Brölemann, 1901. Leptodesmus decipiens, Brölemann N.o $01 \mathrm{e}^{(1)}$. Hab.: (1) Brésil; Paraná. Mus. Paul.: Est. Paraná (Type).

178. Leptodesmus decolor (Humbert of Saussure), 1870.

Leptodesmus decolor, Attems N. $98 \mathrm{~b}^{(1)}$. Polydesmus decolor, Humb. \& Sauss. N. 70.

- (Oxyurus) decolor, Humb. \& Sauss. Hab.: (1) Brésil. N. $72^{(1)}$. 
179. Leptodesmus deerrans Brölemann, 1901.

Leptodesmus deerrans, Brölemann N. 01 e (1). Hab. : (1) Brésil; Alto da Serra (S. P.).

Mus. Paul.: Est. S. Paulo, Alto da Serra (Type).

180. Leptodesmus dilatatus (Brandt) 1839.

Leptodesmus dilatatus, Attems N. 98 b ${ }^{(2)}$. Oxyurus Henselii, Karsch N: 81 c $^{(3)}$. Polydesmus dilatatus, Brandt N. ${ }^{0} 39 \mathrm{a}^{(1)}, 41 \mathrm{~d}^{(1)}$.

\begin{tabular}{|c|c|c|}
\hline - & - & Gray N.: $44^{(1)}$. \\
\hline- & $\therefore$ & Gervais N. ${ }^{\circ} 47$ a $^{(1)}, 59^{(1)}$. \\
\hline & (Oxyurus) & dilatatus, Peters N.o $64^{(1)}$. \\
\hline & - & Karsch N. \\
\hline
\end{tabular}

Non syn.: Polydesmus dilatatus Mikan.

(Karsch-81 c--met le $L$. carneus Saussure en synonymie avec le $L$. dilatatus Brandt; mais le fait n'est nullement prouvé.)

Hab.: (1) Brésil.- ${ }^{(2)}$ Serra Geral.- (3) Sta. Cruz.

181. Leptodesmus eimeri Attems, 1898.

Leptodesmus (Odontopeltis) Eimeri, Attems N." $98 \mathrm{~b}^{(1)}$.

Hab. : (1) Blumenau, Est. Sta. Catharina.

182. I eptodesmus expansus Brölemann, 1902.

Leptodesmus expansus, Brölemann N.0 $02 \mathrm{e}^{(1)}$.

Hab.: (1) Brésil ; Pernambuco.

18:3. I eptodesmus fallax (I'eters), 1864.

Polydesmus (Oxyurus) fallax, Peters N. $64^{(1)}$. Attems N.' 89 b ${ }^{(1)}$. 
Hab.: (1) Brésil; Sta. Cruz.

18t. Leptodesmus forceps Brölkmann, 1901.

Leptodesmus forceps, Brölemann N.o $01 \mathrm{e}^{(1)}$. Hab.: (1) Brésil; Itapetininga (S.P), Alto da Serra (S.P).

Mus. Paul.: Est. S. Paulo, Itapetininga, Alto da Serra (Types).

18.̃. Leptodesmus furcilla Brölemann, 1901.

Leptodesmus furcilla, Brölemann N.0 $01 \mathrm{c}^{(1)}$. Hab.: (1) Brésil; Alto da Serra (S.P). Mus. Paul.: Est. S. Paulo, Alto da Serra (Type).

186. Leptodesmus gibbula Brölemann, 1902.

Leptodesmus gibbula, Brölemann N.0 $02 \mathrm{e}^{(1)}$ Hab.: (1) Brésil ; Pernambuco.

187. Leptodesmus gibbus Brölemann, 1902.

Leptodesmus gibbus, Brölemann N.0 $02 \mathrm{e}^{(1)}$. Hab.: (1) Brésil; Sto. Antonio da Barra (Bahia).

188. Leptodesmus glabratus (Perty), 1833.

Oxyurus glabratus, C. Koch N. 47, $633^{(1)}$. Polydesmus glabratus, Perty N. 33.

Gervais N. $37 \mathrm{a}^{(1)}, 47 \mathrm{a}^{(2)}$, $59 \stackrel{(2)}{.}$

Brandt N.0 $39 \mathrm{a}^{(2)}, 41 \mathrm{~d}^{(2)}$. Lucas N. $40 \mathrm{~b}^{(1)}$. 
Hab.: (1) Brésil. - (2) Depuis l'embouchure du Rio Negro jusqu'aux frontières du Brésil.

189. Leptodesmus gounellei Brölemann, 1902.

Leptodesmus Gounellei, Brölemann N. $02 \mathrm{e}^{(1)}$. Hab.: (1) Brésil; Sto. Antonio da Barra (Bahia).

190. Leptodesmus gracilipes (Humbert \& Saussure), 1870 .

Leptodesmus (Odontopeltis) gracilipes, Attems N." $98 \mathrm{~b}^{(1)}$.

Polydesmus gracilipes, Humb. \& Sauss. N. 70. -- - (Oxyurus) gracilipes, Humb. \& Sauss. N." $72^{(1)}$.

Hab.: (1) Brésil.

191. Leptodesmus iheringi Brölemann, 1901.

Leptodesmus Iheringi, Brölemann N.0 $01 \mathrm{e}^{(1)}$. Itab.: (1) Brésil ; Piquete (S.P). Mus. Paul.: Est. S. Paulo, Piquete (Type).

Leptodesmus (Odontopeltis) incisus =- Leptodesmus (Pseudoleptodesmus) rubescens.

192. Leptodesmus infaustus Brölemann, 1901.

Leptodesmus infaustus, Brölemann N.o $01 \mathrm{e}^{(1)}$. Hab.: (1) Brésil; Os Perus (S.P.). Mus. Paul.: Est. S. Paulo, Os Perís (Type).

193. Leptodesmus jucundus Brileman, 1901. Leptodesmus jucundus, Brölemann N. 01 e (1), (Icon. 03 a). 
Hab.: Brésil ; Sta. Rita (S.P.).

Mus. Paul.: Est. S. Paulo, Santa Rita (Type).

194. Leptodesmus lamellosus Brölemann, 1901.

Leptodesmus lamellosus, Brölemann N.001 $\mathrm{e}^{(1)}$. Hab.: (1) Brésil; Itatiba (S.P.).

Mus. Paul.: Est. S. Paulo, Itatiba (Type).

195. Leptodesmus lartiguei (Silvestri), 1897.

Catharodesmus Lartiguei, Silvestri N.o $97 \mathrm{e}^{(1)}$. Leptodesmus Lartiguei, Attems N.0 99 b ${ }^{(1)}$. Hab.: (1) Pernambuco.

196. Leptodesmus lativentris Brölemann, 1902. Leptodesmus lativentris, Brölemann N.o $02 \mathrm{e}^{(1)}$. Hab.: (1) Brésil; Sto. Antonio da Barra (Bahia).

197. Leptodesmus nattereri (Humbert \& Saussure) 1870.

Leptodesmus Nattereri, Attems N. 98 b (1). Polydesmus Nattereri, Humb. \& Sauss. N. 70. - (Oxyurus) Nattereri, Humb. \& Sauss.

Hab.: Brésil. N. $72^{(1)}$.

198. I Leptodesmus ornj.thopus Brölemann, 1901. Leptodesmus ornithopus, Brölemann N. $01 \mathrm{e}^{(1)}$. Hab.: Brésil; Cerqueira Cezar (S. P.). 
Mus. Paul.: Est. de S. Paulo, Cerqueira Cezar (Type).

199. Leptodesmus pallidus C. Koch, 1847.

Oxyurus pallidus, C. Koch N. $47^{(1)} 63^{(1)}$. Attems $\mathrm{N} .98 \mathrm{~b}{ }^{(1)}$.

Hab.: (1) Brésil.

200. Leptodesmus parmatus Karsch, 1881.

Oxyurus parmatus, Attems N. 98 b (1).

Polydesmus (Oxyurus) parmatus, Karsch N.o 81 c ${ }^{(1)}$.

Hab.: (1) Serra-Geral.

201. Leptodesmus paulistus Brölemann, 1901.

Leptodesmus paulistus, Brölemann N. $01 \mathrm{e}^{(1)}$.

(Peut-être identique au Leptodesmus nudipes Attems, 1898, N. 98 b, Paraguay.)

Hab.: (1) Brésil; Cubatão, (S. P.), Alto da Serra (S. P).

Mus. Paul.: Est. de S. Paulo, Alto da Serra, Cubatão, (Types).

202. Leptodesmus (Strongylosomides) petropolites Attems, 1901.

Leptodesmus (Strongylosomides) petropolites, Attems $\mathrm{N}^{\circ} 01 \mathrm{e}^{(1)}$.

(N'est peut-ître qu'une variété de Leptodesmus: cognatus Brölemann.)

Hab.: (1) Brésil ; Petropolis. 
203. Luptodesmus (? Rachidomorpha) rosascens . Brandt, 1839.

Poìdesmus rosascens, Brandt N. $39 \mathrm{a}^{(1)}, 41 \mathrm{~d}^{(1)}$. - rosasceus, Gervais N. $47 \mathrm{a}^{(1)}, 59^{(1)}$. - (Rachidomorpha) rosascens, Peters N. 64 (1).

Rachidomorpha rosascens, Attems, N. 98 b ${ }^{(1)}$. Hab.: (1) Hrésil.

204. Leptodesmus (Pseudoleptodesmus) rubescens (Gervais), 1836.

Leptodesmus (Odontopeltis) incisus, Attems N.。 $98 \mathrm{~b}^{(2)}$.

Leptodesmus (Pseudoleptodesmus) rubescens, Brölemann N:0 $01 \mathrm{e}^{(1)}$.

Polydesmus rubescens, Gervais N. 36 b ${ }^{(1)}$, $37 \mathrm{a}^{(1)}, 47 \mathrm{a}^{(1)}, 59^{(1)}$. - - Brandt N. $39 \mathrm{a}^{(1)}$, $41 \mathrm{~d}^{(1)}$.

- - Lucas N. 40 b ${ }^{(1)}$.

- (Rhacophorus) rubescens, Peters N. $64^{(1)}$.

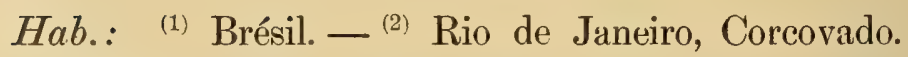

205. I eptodesmus simillimus Brölemann, 1902. Leptodesmus simillimus, Brölemann N.o $02 \mathrm{e}^{(1)}$. Hab.: (1) Brésil; Sto. Antonio da Barra (Bahia).

206. Leptodesmus (Rachidomorpha) tarascus (Saussure), 1860.

Leptodesmus (Rachidomorpha) tarascus, Attems N. 01 e ${ }^{(1)}$.

Polydesmus tarascus, Saussure N. $60^{(3)}$. 
Rachidomorphai tarascus, Attems N.o 98 b ${ }^{(2.3)}$.

Hab.: (1) Brésil ; Espirito Santo.--(2) Cordova. ${ }^{(3)}$ Mexique.

207. Leptodesmus tuberculiporus Attems, 1898.

Leptodesmus tuberculiporus, Attems N. $98 \mathrm{~b}^{(1)}$. Hab.: Bahia?

208. Leptodesmus vanvolxemi (Silvestri), 1897.

Strongylomorpha van-Volxemi, Silvestri N.o $97 \mathrm{e}^{(1)}$. Strongylosoma van-Volxemi, Attems N. 90 b ${ }^{(1)}$. Hab.: (1) Pedro Açu.

209. Leptodesmus verrucula Brölemann, 1901.

Leptodesmus verrucula, Brölemann N.001 $\mathrm{e}^{(1)}$. $H a b$. : (1) Brésil; Alto da Serra (S. P.).

Mus. Paul.: Est. de S. Paulo, Alto da Serra (Type).

210. Leptodesmus virgulatus Attems, 1901.

Leptodesmus virgulatus, Attems N.01) $1 \mathrm{c}^{(1)}$. Hab.: (1) Brésil; Petropolis.

211. Leptodesmus volutatus Brölemann, 1901.

Leptodesmus volutatus, Brölemann N.o $01 \mathrm{e}^{(1)}$.

Hab.: (1) Brésil ; Itapetininga (S. P.), Belem (S. P), Alto da Serra (S. P.).

Mus. Paul.: Est. de S. Paulo, Itapetininga (Type), Cerqueira Cezar, Alto da Serra, 
212. Leptodesmus zelebori (Humbert \& Saussure), 1870.

Leptodesmus Zelebori, Attems N. 98 b $^{(1)}$. Polydesmus Zelebori. Humb. \& Sauss. N. 70. - (Oxyurus) Zelebori, Humb.\&Sauss. N. $72^{(1)}$.

Hah.: (1) Rio de Janeiro.

38. Genre: ODONTOTROPIS IIumbert \& Saussure, 1869.

213. Odontotropis clarazianus (Humbert of Saussure), $186 \%$.

Odontotropis Clarazianus, Attems N. $98 \mathrm{~b}^{(2)}$. Brölemann N. $02 \mathrm{f}^{(1)}$. Polydesmus (Odontotropis) Clarazianus, Humb. \& Sauss. N. $69 \mathrm{~b}^{(2)}, 72^{(2)}$. - - Clarazianus, Brölemann N. 00 a $^{(1)}$.

Hab.: (1) Therezopolis (R. de Jan.).-- ${ }^{(2)}$ Argentine.

39. Genre: ORTHOMORPHA Bollman, 1893.

214. Orthomorpha coarctata (Saussure), 1860. Orthomorpha coarctata, Bollman N. $93^{(4)}$.

\begin{tabular}{|c|c|c|}
\hline - & - & Pocock N.. $95 \mathrm{c}^{(11)}, 00 \mathrm{~d}^{(14)}$. \\
\hline & - & Silvestri $\mathrm{N}^{\circ} 96 \mathrm{~b}^{(1)}, 96 \mathrm{e}^{(6)}$. \\
\hline & - & $\begin{array}{l}\text { Brölemann } \mathrm{N}^{0} \quad 96 \mathrm{~d}^{(9)}, \\
98 \mathrm{e}^{(5)}, 02 \mathrm{f}^{(11)}, 03 \mathrm{a}^{(2)} .\end{array}$ \\
\hline & - & $\underset{(3-7-11-12-13)}{\operatorname{Attems} \mathrm{N}} .97 \mathrm{a}^{(11)}, 98 \mathrm{~b}$ \\
\hline & $i a$, & Cook N. $96 \mathrm{e}^{(10)}$. \\
\hline
\end{tabular}


Paradesmus coarctatus, Tömösvary N. $85^{(11)}$.

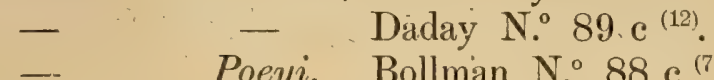

Polydesmus coarctatus, Saussure N. $60^{(3)}$.

Peters N. 64 .

- (Paradesmus) coarctatus, Humb. \& Sauss. N. $69 \mathrm{a}^{(11)}$.

- - vicarius, Karsch N. $81 \mathrm{e}^{(11)}$.

Strongylosoma coarctatum Pocock N.o $93 \mathrm{a}^{(7.8)}, 93 \mathrm{e}^{(7)}$, $94 \mathrm{~b}^{(11)}$.

- Poeyi, Bollman N. $87 \mathrm{~g}^{(7)}$.

Hab.: (1) Pará.-(2) Manáos- ${ }^{(3)}$ Cayenne.- ${ }^{(4)}$ Paramaribo -- Surinam.- ${ }^{(5)}$ Venezuela.- ${ }^{(6)}$ Colombie. - ${ }^{(7)}$ Antilles._- ${ }^{(8)}$ Bermudes._- ${ }^{(9)}$ Sechelles.- ${ }^{(10)}$ Liberia, Monrovia. - ${ }^{(11)}$ Asie et Malaisie. - (12) Borneo._- ${ }^{(13)}$ Madagascar. - ${ }^{(14)}$.Christmas Island. Mus. Paul.: Est. Amazonas, Manáos.

215. Orthomorpha gracilis (C. Koch), 1847.

Fontaria gracilis, Orthomorpha gracilis,

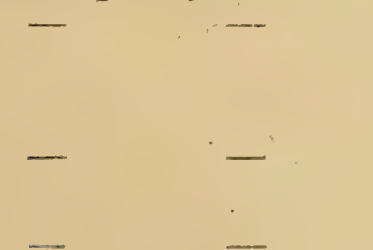

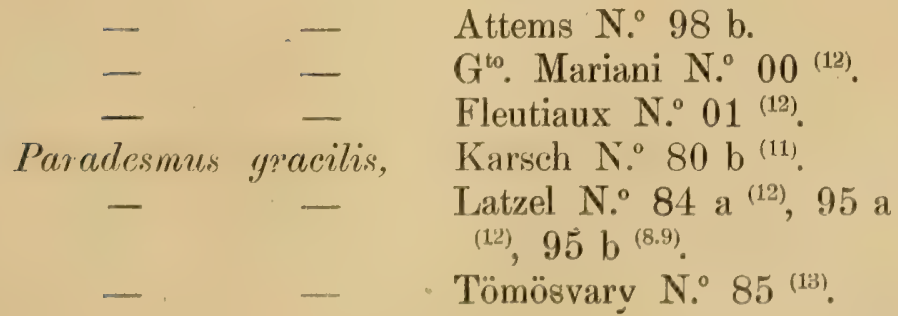

C. Koch N. $47^{(13)}, 63^{(13)}$. Pocock N. 95 a ${ }^{(13)}$. $96 \mathrm{~d}^{(11)}, 97 \mathrm{~b}^{(8)}, 00 \mathrm{~g}{ }^{(7)}$, $01 \mathrm{e}^{(4)}$.

Verhoeff $\mathrm{N}^{\circ} 97 \mathrm{e}^{(12)}, 01$ $\mathrm{f}^{(12)}$.
Brölemann N. $96 \mathrm{e}^{(12)}$, 
Paradesmus gracilis, Pocock N. $88 \mathrm{e}^{(5)}$.

\begin{tabular}{|c|c|c|}
\hline - & - & Porat N. $89^{(12)}$. \\
\hline - & - & $\begin{array}{l}\text { Daday } \mathrm{N}^{\circ} 89 \mathrm{a}^{(12)}, 89 \\
(14), 91^{(15)} .\end{array}$ \\
\hline & & Ellingsen $\mathrm{N}^{\circ} 92^{(12)}$. \\
\hline & & Brölemann N. $95 \mathrm{c}^{(12)}$. \\
\hline & & Ihering N. $95^{(3)}$. \\
\hline & & Storm N. ${ }^{\circ} 98^{(12)}$. \\
\hline
\end{tabular}

Polydesmus (Paradesmus) gracilis, Porat N. 72 (10). Lendl N. $94^{(13)}$. Strongylosoma gracile, Pocock N. 93 a $\stackrel{(6)}{.}$.

$H a b:{ }^{(1)}$ Rio de Janeiro.- ${ }^{(2)}$ Santos.- ${ }^{(3)}$ São Paulo. - ${ }^{\text {(4) }}$ Brésil; Alto da Serra Piquete, Cubatão, Sta. Rita, Santos, Belém. (S.P.); Paraná. - ${ }^{(5)}$ La Dominique.- ${ }^{(6)}$ Bermudes.- ${ }^{(7)}$ Guatemala.${ }^{(8)}$ Madère.- ${ }^{(9)}$ Canaries. - ${ }^{(10)}$ Afrique.- ${ }^{(11)}$ Sechelles.- ${ }^{(12)}$ Europe.- ${ }^{(13)}$ Asie et Malaisie.- ${ }^{(14)}$ Borneo. - ${ }^{(15)}$ Samoa.- ${ }^{(16)}$ Olinda.

Mus. Paul.: Est. S. Paulo, Santos, Cubatão, Alto da Serra, Belem, Piquete, Santa Rita; Est. Paraná.

Oxyurus cinerascens = Leptodesmus cinerascens. Oxyurus fallax = Leptodesmus fallax.

Oxyurus glabratus = Leptodesmus glabratus. Oxyurus henselii $=$ Leptodesmus dilatatus. Oxyurus pallidus = Leptodesmus pallidus. Oxyurus parmatus $=$ Leptodesmus parmatus. Paradesmus coarctatus = Orthomorpha coarctata. Paradesmus gracilis = Orthomorpha gracilis. Paradesmus poeyi $=$ Orthomorpha coarctata. 
40. Genre: PLATYRRhacUs C. Koch, 1847.

216. Platyrrhacus bilineatus (Lucas), 1840.

Platyrrhacus bilineains, Attems N. 99 b (1). Polydesmus bilineatus, Lucas $\mathrm{N} .{ }^{\circ} 40 \mathrm{~b}{ }^{(2)}$. Gervais N.0 47 a ${ }^{(2)}, 59^{(2)}$. - (Stenonia) bilineatus, Saussure N.o $60^{(2)}$. Hab.: (1) Amazones; Perú; Olivença.--(2) Mexique.

217. Platyrrhacus rufipes C. Koch, 1847.

Platyrrhacus rufipes, C. Koch N. $47^{(1)}, 63^{(1)}$. Aab.: (1) Provenance incertaine, probablement du Brésil.

218. Platyrrhacus scaber (Perty), 1833.

Platyrrhacus scaber, C. Koch N.0 $47^{(1)}, 63^{(1)}$. Polydesmus scaber, Perty N.o 33.

- $\quad$ Gervais N.0 $37 \mathrm{a}^{(1)}$.
- $\quad$ Brandt N.0 $39 \mathrm{a}^{(2)}, 41 \mathrm{~d}^{(2)}$.

(Humbert \& Saussure considèrent l'espèce de Perty comme differente de celle de C. Koch.)

Hab.: (1) Brésil.-( ${ }^{(2)}$ Est. de Minas Geraes.

\section{1.: Genre POLYDESWUS Latreille, 1802.}

( ( )n n'a trouvé jusqu'ici aucun véritable représentant de ce genre au Brésil. Les espèces qui figurent encore clans notre liste appartiennent certainement à d'autres genres; mais elles ont été si brièvement décrites qu'on ne pourra probablement jamais leur assigner leur véritable place.) 
219. Polydesmus abbreviatus (Mikan), 1834.

Iulus abbreviatus, - Mikan, N. $34^{(2)}$. Polydesmus abbreviatus, Gervais N. $47 \mathrm{a}^{(1)}$. Hab.: (1) Brésil. - ${ }^{(2)}$ Rio de Janeiro.

Polydesmus (Rachidomorpha) aduncus = Leptodesmus (Rachidomorpha) aduncus.

220. Polydesmus albipes Newport, 1844.

Polydesmus albipes, Newport (teste Gray N.o $44^{(1)}$ ). Hab.: (1) Brésil.

Polydesmus bilineatus = Platyrrhacus bilineatus. Polydesmus carneus = Leptodesmus carneus. Polydesmus clarazianus = Odontotropis clarazianus. Polydesmus coarctatus = Orthomorpha coaretata. Polydesmus (Strongylosoma) concolor = Strongylosoma concolor.

Polydesmus conspersus = Leptodesmus conspersus.

221. Polydesmus dasypus Gervais, 1847.

Polydesmus dasypus, Gervais N. 47 a, $59^{(1)}$.

Hab.: (1) Amérique méridionale.

Polydesmus decolor $=$ Leptodesmus decolor.

222. Polydesmus dentosus (Mikan), 1834.

Iulus dentosus, Mikan N. $34^{(1)}$. Polydesmus dentosis, Gervais N. 47 a ${ }^{(1)}$. Hab.: (1) Brésil. 
223. Polydesmus dilatatus (Mikan); 1834.

Iulus dilatatus, Mikan N. $34^{(1)}$.

Polydesmus dilatatus, Gervais N.0 $47 \mathrm{a}^{(1)}$.

Non syn.: Polydesmus dilatatus Brandt = Leptodesmus dilatatus.

Hab.: (1) Brésil.

Polydesmus fallax = Leptodesmus fallax.

224. Polydesmus flavipes (Mikan), 1834.

Iulus flavipes, Mikan N. $34^{(1)}$.

Polydesmus flavipes, Gervais N. 47 a ${ }^{(1)}$.

Hab.: (1) Brésil; environs de Rio de Janeiro.

Polydesmus glabratus = Leptodesmus glabratus.

Polydesmus gracilipes = Leptodesmus gracilipes.

Polydesmus gracilis = Orthomorpha gracilis.

225. Polydesmus hamulosus (Mikan), 1834.

Iulus hamulosus, Mikan N. $34^{(1)}$. Polydesmus hamulosus, Gervais N. $47 \mathrm{a}^{(1)}$.

Hab.: (1) Brésil.

Polydesmus nattereri = Leptodesmus nattereri.

Polydesmus olfersi $=$ Cryptodesmus olfer $\mathbf{~} \mathrm{i}$.

Polydesmus parmatus -. Leptodesmus parmatus.

2261. Polydesmus pinnatus (Mikan), $18,34$.

Iulus pinnatus, Mikan N.0 $34^{(1)}$. Polydesmus pinnatus, Gervais, N.o 47 : ${ }^{(1)}$. 
Hab.: (1) Brésil.

Polydesmus rosascens seu rosasceus = Leptodesmus (? Rachidomorpha) rosascens.

Polydesmus rubescens = Leptodesmus (Pseudoleptodesmus) rubescens.

227. Polydesmus rugulosus Eschscholti, 1823.

Polydesmus rugulosus, Eschscholtz N. 23.

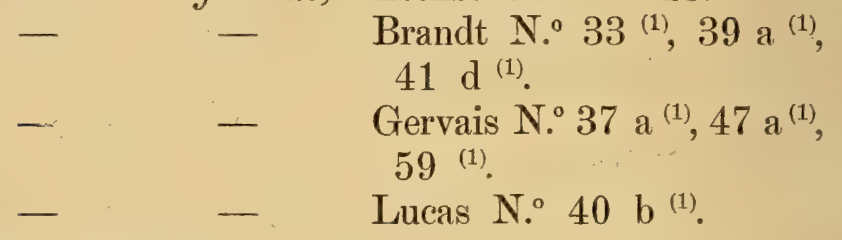

Hab.: (1) Brésil.

Polydesmus scaber = Platyrrhacus scaber.

228. Polydesmus serrulatus (Mikan), 1834.

Iulus serrulatus, Mikan N. $34^{(1)}$.

Polydesmus sermulatus, Gervais N. $47 \mathrm{a}^{(1)}$.

Hab.: (1) Brésil.

Polydesmus tarascus = Leptodesmus (Rachidomorpha) tarascus.

229. Polydesmus tuberculosus (Mikan), 18.34.

Iulus tuberculosus, Mikan N. $34^{(2)}$.

Polydesmus tuberculosus, Gervais N. 47 a ${ }^{(1)}$.

Hab.: (1) Brésil.- (2) Rio de Janeiro.

Polydesmus uncinatus = Leptodesmus (Rachidomorpha) aduncus. 
Polydesmus vicarius - Orthomorpha coarctata.

Pulydesmus zebratus seu zonatus -- Fontaria zebrata. Polydesmus zelebori $=$ Leptodesmus zelebori

42.e Genre: PRIODESIIUS Cook, 1895.

230. Priodesmus parae Cook, 1895.

Priodesmus parae, Cook N. $95 \mathrm{f}^{(1)}$.

- - Attems N. $99 \mathrm{~b}^{(1)}$.

Hab.: (1) Pará.

Rashidomorpha aduncus = Leptodesmus (Rachidomorpha) aduncus.

(La description donnée par Attems- - 01 c —des Pattes Copulatrices du $R$. tarascus semble trancher la question de la place que doit occuper ce groupe dans la classification. Il y a cependant lieu de faire exception pour le $R$. brasiliae dont les P. C. sont d'un type autre que celles de $R$. tarascus).

Rachidomorpha bicolor = Leptodesmus bicolor.

Rachidomorpha brasiliae = Leptodesmus brasiliae. Rachidomorpha rosasiens -- Leptodesmus (Rachidomorpha) rosascens.

Rachidomorpha tarascus =- Leptodesmus (Rachidomorpha) tarascus.

Rhacophorus conspersus Leptodesmus conspersus.

Strongylomorpha van-uolicmi - - I Leptodesmus vairvolxemi. 
43.. Genre: STRONGYLOSOMA Brandt, 1833.

231. Strongylosoma apexgaleae Brölemann, 1901.

Strongylosoma apex-galeae, Brölemann N.o $01 \mathrm{e}^{(1)}$. Hah.: Brésil ; Belém. (S. P.), Alto da Serra (S. P.). Mus. Paul.: Est. S. Paulo, Belém, Alto da Serra (Type).

Strongylosoma coarctatum $=$ Orthomorpha coarctata.

232. Strongylosoma concolor Gerrais, 1847.

Polydesmus (Strongylosoma) concolor, Peters N. $64^{(1)}$.

Strongylosoma concolor, Gervais N.o 47 a ${ }^{(2)}, 59^{(2.3)}$. Hab.: (1) Brésil.- ${ }^{(2)}$ Coquimbo.- ${ }^{(3)}$ Montevidéo (?).

233. Strongylosoma coniterum Attems, 1898.

Strongylosoma coniferum, Attems N. $98 \mathrm{~b}^{(1)}$.

Hab.: (1) Brésil; Blumenau, Santa Catharina.

234. Strongylosoma drepanephoron Attems, 1898.

Strongylosoma drepanephoron, Attems N. $98 \mathrm{~b}^{(1)}$. Brölemann N.o 02 $\mathrm{e}^{(2)}$.

Hab.: (1) Santos.-_(2) Brésil ; Fazenda Nova Nicaragua (S. P.).

235. Strongylosoma glabrum Petter's, 1864.

Strongylosoma glabrum, Peters N. $64^{(2)}$.

- Karsch N. $81 \mathrm{c}^{(1)}$.


Strongylosoma glabrum, Attems N. $98 \mathrm{~b}^{(1)}$. Hab.: (1) Serra Geral.— ${ }^{(2)}$ Colombie. Strongylosoma gracile = Orthomorpha gracile.

236. Strongylosoma kalliston Attems, 1898.

Strongylosoma kalliston, Attems N.0 $98 \mathrm{~b}^{(1)}$. Hab.: (1) Rio Grande do Sul, S. Cruz.

237. Strongylosoma levisetum Attems, 1898. Strongylosoma levisetum, Attems $\mathrm{N} .{ }^{\circ} 98 \mathrm{~b}^{(1)}$. Hab.: (1) Brésil ; Blumenau, Santa Catharina.

238. Strongylosoma mesorphinum Attems, 1898.

Strongylosoma mesorphinum, Attems N. ${ }^{\circ} 98 \cdot b^{(1)}$. Hab.: (1) Brésil ; Santa Catharina.

239. Strongylosoma mesoxanthum Attems, 1898.

Strongylosoma mesoxanthum, Attems N. $98 \mathrm{~b}^{(1)}$. Hab.: (1) Brésil; Blumenau, Santa Catharina.

240. Strongylosoma myrmekurum Atteins, 1898.

Strongylosoma myrmekurum, Attems $\mathrm{N}^{\circ} 98 \mathrm{~b}^{(1)}$. Hab.: (1) Brésil; Blumenau, Santa Catharina.

241. Strongylosoma nitidum Brölemann, 19011.

Strongylosoma nitidum, Brölemann N:01 $\mathrm{e}^{(1)}$. Hab.: (1) Brésil; Cubatāo (S. P.). 
Mus. Paul.: Est. S. Paulo, Cubatão (Type).

242. Strongylosoma paraguayense Silvestri, 1895.

Strongylosoma paraguayense, Silvestri N. ${ }^{\circ} 95 \mathrm{~d}^{(2)}$. Attems N. $98 \mathrm{~b}^{(1)}$.

Hab.: (1) Brésil; Blumenau, Santa Catharina.-(2) Argentine.

Strongylosoma poeyi $=$ Orthomorpha coarctata.

243. Strongylosoma pustulatum Bröleman, 1902.

Strongylosoma pustulatum, Brölemann N.o $02 \mathrm{e}^{(1)}$. Hab.: (1) Brésil; Fazenda Nova Nicaragua (S. P.).

244. Strongylosoma spilonotum Gervais, 1847.

Strongylosoma spilonotum, Gervais N. 47 a ${ }^{(1)}, 59^{(1)}$. Hab.: Amérique méridionale.

Strongylosoma van-volxemi $=$ Leptodesmus vanvolxemi.

Ce catalogue était déjà préparé pour l’impression lorsque nous parvint le dernier travail du Dr. Silvestri «Viaggio del Dott. A. Borelli nel Matto Grosso» (Boll. Mus. Zool. Anat. comp. Torino, XVJI, N. 432, 26 Dicembre 1902) dans lequel l'auteur signale, comme trouvées au Brésil, les espèces suivantes:

\section{SPIROBOLIDAE}

245. Rhinocricus nodulipes Silvestri, 1897.

Hab.: Urucum (M. Grosso). 


\section{SPIROSTREPTIDAE}

246. Arohispirostreptus (1) libertinus Silvestri, 1895.

Hab.: Corumbá (M. Grosso).

247. Diaporus amexicanus perproximus Silvestri, 1902.

Hab.: Carandásinho (M. Grosso).

248. Nanostreptus mattogrossensis Silvestri, 1902.

Hab.: Corumbá, Carandásinho, Itaicy (M. Grosso).

249. Odontopyge (2) paraguayensis Silvestri, 1895.

Hab.: Urucum, Itaicy (M. Grosso).

250. Plusioporus gigliotosi Silrestri, 1902.

Hab.: Corumbá (M. Grosso).

\section{PSEUDONANNOLENIDAE}

251. Pseudonannolene brevis Silvestri, 1902. Hab.: Paraná.

252. Pseudonannolene parvula Silnestri, 1902. Hab.: Bella Vista (Par.).

253. Pseudonannolene typica Silvestri, 1895 Hab.: Bella Vista (Par.).

(1) Sons le nouveau nom de: Nanostrcptus libertinus.

(2) Sons le nouveau nom de: Heteropyge paraguayensis. 


\section{POLYDESMIDAE}

254. Euthydesmus (1) acicarina Silvestri, 1902. Hab.: Urucum, Carandasinho (M. Grosso).

255. Leiodesmus ${ }^{(2)}$ carcani Silvestri, 1902. Hab.: Urucum (M. G.).

256 Leiodesmus orlandi Silvestri, 1902. Hab.: Carandasinho (M. Grosso).

257. Leptodesmus bohlsi (3) Atterns, 1898. Hab.: Bella Vista (Par.).

258. Leptodesmus ${ }^{(4)}$ parallelus Attems, 1898. Hab.: Urucum (M. Grosso).

259. Mestosoma ${ }^{(5)}$ bicolor Silvestri, 1898. $H a b$. : Urucum (M. Grosso).

260. Sandalodesmus ${ }^{(6)}$ bertonii Silvestri, 1902. Hab.: Bella Vista (Par.).

(I) Genre non caracterisé: peut-être un Leptodesmus.

(2) Nom de genre condamné par Attems et tombant en synonymie avec Leptodesmus.

(3) Sous le nom de Erythrodesmus Bovei (Odontopeltis bovei) qui n'a jamais été suffisemment caracterisé pour être reconnaissable et doit par conséquent céder le pas au nom créé par Attems.

(4) Sous le nom nouveau, mais en aucune façon justifié, de: Brachyurodesmus parallelus.

(5) Nom de genre condamné par Attems et tombant en synonymie avec Strongylosoma.

(6) Genre non caracterisé; nomen nudum. 


\section{INDEX ALPHABETIQUE}

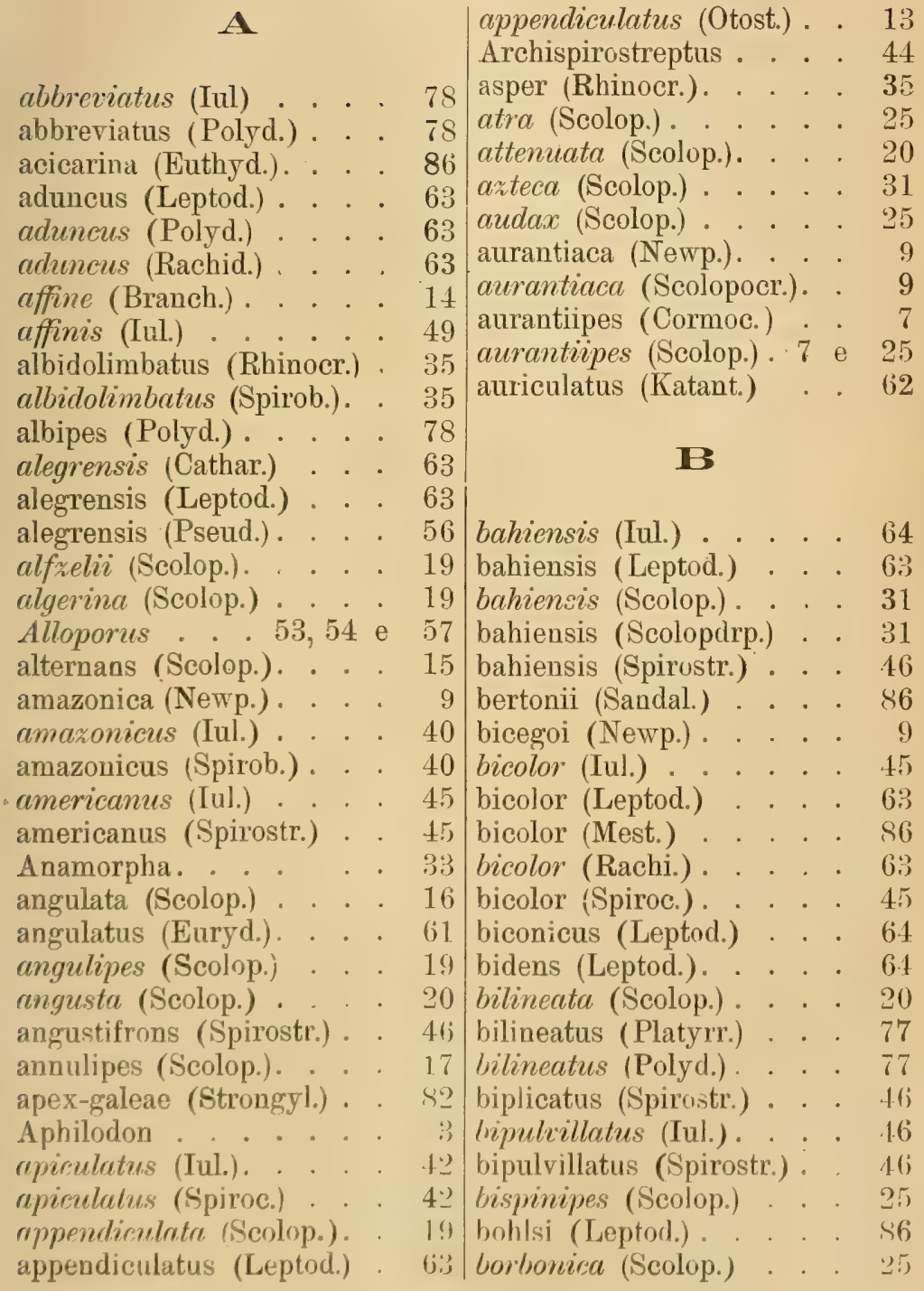


bovei (Erythrod.) . . . . $86 \mid$ cephalica (Scolop.). . . . 25

bovei (Odont.).. . . . . 86 ceylonensis (Scolop.) . . . 26

brachypoda (Scolop.). . . 20 childreni (Scolop.). . . . 26

Brachyurodesmus . . . 86 chlorocephala (Scolop.) . . 20

Branchyotrema . . . . 13 Chomatobius . . . . . 3

brandtiana (Scolop.) . . . 20 cinctus (Spirostr.) . . . . 47

brasiliae (Leptod.)... . . 64 cinerascens (Leptod.). . . 65

brasiliae (Rachid.) , 64 e 81 cinerascens (Ox.) . . . . 65

brasiliana (Schend.) . . . 6 cingulata (Scolop.). . . . 20

brasilianus (Chomat.). . . 3 Cladostreptus. . . . 46 e 54

brasilianus (Nann.) . . . 6 clarazianus (Odont.) . . . 74

brasitiensis (Cormoc.) . . 8 clarazianus (Polyd.) . . . 74

brasiliensis (Crypt.) . . . 8 clavaria (Leptod.) . . . . 65

brasiliensis (Cup.). . . . 8 cluniculus (Spirostr.). . . 47

brasiliensis (Orph.) . . . 5 coarctata (Orth.). . . . . 74

brasiliensis (Otost.) . . . 13 coarctatum (Strong.) . . . 75

brasiliensis (Siph.)... . 34 coarctatus (Parad.). . . 75

brevilabiatus (Orph.). . . 5 coarctatus (Polyd.). . . . 75

brevis (Pseud.) .. . . . 85 cognata (Scolop.) . . . 20

byssina (Scolop.) . . . 25 cognatus (Leptod.) . . . 65

\section{C}

caicarae (Spirostr.).

calcaratus (Pith.)

calcaratus (S solopdrp.) .

californica (Scolop.) .

callipyge (Pseud.)

callipyge (Pseud.) .

capivarae (Crvpt.) .

carcani (Leiod.) .

carinipes (Scolop.).

carinovatus (Leptod.)

carminatus (Leptod.)'.

carnea (Font.) .

carneus (Leptod.)

carneus (Polyd.).

Catharodesmus . . .63 e

caudatus (Iul.)

caudatus (Otost.) . . .

caudatus (Rhinocr.)

caudatus (Spirob.). .36 e

celer (Branch.)

celeris (Rhys.)

centropus (Leptod.)
46 compressipes (Scolop.) . . 20

cognatus (Leptod.) . . . 71

Colobognatha . . . . 34

complanata (Scolop.). . . 15

32 concinnus (Rhinocr.) . . . 36

32 concolor (Polyd.) . . . . 82

20 concolor (Scolop.) . . . 26

57 concolor (Strong.) . . . 82

57 coniferum (Strong.) . . . 82

8 conspersus (Leptod.) . . . 65

86 conspersus (Polyd.) . . . 65

20 conspersus (Rhac.) . . . 66

64 corcovadis (Leptod.) . . 66

64 Cormocephalus . . . . . 7

62 corniger (Leptod.) . . . . 66

65 crassicornis (Iul.) . . . 59

65 cristata (Scolop.) . . . . 29

70 crudelis (Scolop.) . . . 15

36 cruentatus (Spirob.) . . . 46

12 Cryptodesmus. : . . . 60

36 Cryptops . . . . . . 8

40 Crypturodesmus . . . . 61

14 cultratus (Spirostr.) . . . 47

14 Cupipes . . . . . . 8

65 cylindricus (Leptod.) . . 66 
damnosa (Scolop.) . . . 26 dasypus (Polyd.). . . . . 78 dealbatus (Spirob.) . . . 40 decipiens (Leptod.) . . . . 66 decolor (Leptod.) . . . . 66 decolor (Polyd.). . . . . 66 deerrans (Leptod.) . . . . 67 de-haani (Scolop.) . . . 26 dentosus (Iul.) . . . . 78 dentosus (Polyd.) . . . . 78 deserticola (Spirostr.). . . 47

Diaporus .

dictyonotus (Spirob.) . dilatatus (Iul.) dilatatus (Leptod.) . . . . dilatatus (Polyd.) . . . . dilatatus (Polyd.) . . . . dinodon (Scolop.) . . . . dipticus (Spirostr.). . . . dominicae (Spirob.) . . . 44 dorsostriatus (Spirostr.) . . drepanephoron (Strong.). dubia (Font) .

\section{E}

eimeri (Leptod.). . . . 67

elegans (Heter.) . . . . . 24

elegans (Scolop.). . . . 20

elegans (Scolop. fulvipes) .

elongata (Scolop.)

elongatus (Eur.).

epileptica (Scolop.). . . .

Epimorpha .

ernsti (Newp.) erusti (Scolopdrd.). . . . erythrocephala (Scolop.). Eiythorodesmus Eurydesmus

Eurylithobius .

Euryurus

Euthydesmus .

excavatus (Spirostr.) . expansus (Leptod.).

67

\section{$\mathbf{F}$}

fabricii (Scolop.)

21

fallax (Leptod.) . . . . . 67

fallax (Polyd.) . . . . 67

fartus (Spirostr.). . . . 48

ferruginea (Scolop.) . . . 26

festivus (Iulus) . . . . 48

festivus (Spirob.) . . . 40

47 festivus (Spirostr.) . . . 48

85 filum (Spirostr.) . . . . 48

40 fissispini (Scolop.) . . . 26

79 flava (Scolop.) . . 26 e 29

67 flavipes (Tul.) . . . . . 79

79 flavipes (Polyd.). . . . . 79

67 flavofasciatus (Spirostr.) . 47

26 Fontaria . . . . . . 62

47 forceps (Leptod.) . . . . 68

44 forficatus (Lith.) . . . . 34

48 formosa (Scolop.) . . . 21

82 fulvipes (Heter.) . . . . 24

$62 \mid$ fulvipes (Scolop.) . . . 21

furcilla (Leptod.) . . . . 68

fuscipes (Spirostr.). . . . 49

20 Geophilidae. . . . . 3

21 Geophilus . . . . . 4

26 georgicus (Scolopocr.) . . 12

61 gervaisiana (Scolop.) 21 e 24

18 gervaisii (Scolop.) . . . 26

3 gibbula (Leptod.) . . . 68

10 gibbus (Leptod.). . . . 68

18 gigantea (Scolop.) * 18 e 29

30 gigantea (Scolop.) , ¿6 o 29

81; gigas (Scolop.) . . . . 18

(i). gigliotosi (Plus.). . . . 85

24 glabratus (Leptod.). . . . 68

(;) 1 glabratus (Ox.) . . . . lis

86 glabratus (Polyd.) . . . . 68

ts glabrum (Strong.) . . . 92 
goeldii (Otost.) goesi (Spirob.) . . . . . goesi (Trig.) . . . . . . gounellei (Leptod.). . . . 69 gounellei.(Schend.) . . . 6 gounellei (Spirostr). . . . 49 gracile (Branch.) . . . . 14 gracile (Strong.). . gracilipes (Leptod.) gracilipes (Polyd.) . gracilipes (Scolop.). gracilis (Cormoc.) . gracilis (Font.) gracilis (Orth.) gracilis (Parad.) gracilis (Polyd.). gracilis (Pseud. tricolor) : gracitis (Scolop. cephalica) grandis (Iul.) grandis (Notiph.) . . . . grandis (Spirob.) . . . . grayii (Scolop.) : . . . . griseus (Spirob.). . . . . guillemini (Greoph.)

Gymnostreptus 47, 49, 52,

\section{H}

$54 \mathrm{e}$

hamulosus (Iul.) hamulosus (Polyd.) . . . heilprini (Spirob.) . . . . helvola (Scolopdrp.) . . . Heniorya . . . . . henselii (Ox.) . herculeana (Scolop.) . . . 30 Heteropyge. . . . . . 86 Heterostoma . . . . . . 24 histrionica (Scolop. de-haani) 29 hopei (Scolop.) . . . . 30 horrida (Scolop.) . . . 27

\section{I}

iheringi (Crypt..) iheringi (Leptod.) 40

4
12 iheringi (Spirostr.). . . . 49

36 imperfossa (Schend.) . . . 6

44 impressa (Scolop.) . . . . 21

44 impressus (Spirob.). . . . 41

9 inaequidens (Scolop.). . . 16

incerta (Scolop.). . . . .. 16

incissus (Leptod.) . . . . 72

indus (Iul.). . . . . . . 50

indus (Spirostr.). . . . 49

69 inermis (Pith.) . . . . . 31

69 inermis (Scolop.) . . . . 27

26 infaustus (Leptod.). . . . 69

7 infesta (Scolop.). . . . 21

75 insignis (Scolop.) . . . . 18

75 insulsus (Rhinocr.). . . 36

75 intermedia (Scolop.) . . . 21

76 interruptus (Spirostr.) . . 50

58 Iulidae . . . . . . . . 58

25 Iulus . . . . . . 58

15 jucundus (Leptod.). . . . 69

41 Juloidea . . . . . . 35

lativentris (Leptod.) . 70

leachii (Scolop.) . . . . . 21

Leiodesmus. . . . . 8ti

leptodera (Scolop.). . . . 19

Leptodesmus . . . . . 62

levior (Spirostr. perlucens) 52

levisetum (Strong.) . . . 83

69 libertinus (Archisp.) . . . 85 
libertinus (Nan.) . . . 86

limbutus (Otost.). . . . 13

limbatus (Rhinocr.) . . . 36

limisolor (Seolop.). . . . 27

lineatus (Orph.).

Lithobiidae.

Lithobius

lobulatus (Spirostr.)

lobulatus (Arch.) . . . .

longiceps (Scolopocr.) . .

longicornis (All.) . . . .

longicornis (Pseud.) . . .

longicornis (Scolop.) . . .

longipes (Branch.). . . . 14

longipes (Thys.). . . . 14

longipes (Scolop.) . . . . 16

longipleura (Scolop.). . . 19

longispina (Scolop.) . . . 19

longissima (Hen.) . . . . 4

longitarsis (Newp.). . . 10

longitarsis (Scolopocr.) , . 10

lucasii (Scolop.). . . . . 27

lutea (Scolop.) . . . . 27

\section{$\mathbf{M}$}

macracanthus (Scolop.). .

macrourus (Spirob.)

mactans (Scolop.)

marginata (Scolop.)

marginatus (Cormoc.)

marginelius (Rhinocr.) .

marus (Spirostr.)

mathematicus (Spirostr.) .

mattogrossensis (Nanostr.).

maximus (Iul.)

maximus (Spirob.) .

maximus (Spirocycl.). . .

maya (Scolop.) . . . .

megacephalus (Scolopocr.).

melanosoma (Scolopocr.) .

melanostoma (Otocr.).

melanostoma (Scolopocr.).

mellitus (Spirostr.) .

mesorphinum (Strong.) .

mesoxanthum (Strong.) .

10

10
Mestosoma . . . . . 86

micronyx (Aph.). . . . . 3

microps (Spirostr.) . . . 51

miersi (Scolopocr.) . . . . 33

miersii (Scolopocr.) . . . 33

mitis (Cup. ungulata). . . 9

moestus (Rhinocr.). . . . 37

monilicornis (Rhinocr.) . . 37

monilicornis (Spirob.) . . 37

morsicans (Scolop.) . . . 22

morsitans (Scolop.). . . . 19

mossambica (Scolop.). . . 22

multispinata (Scolop.) : . 16

multispinosa (Scolop.) . . 16

multispinosa (Scolo. morsitans). . . . . 24

Myriapoda-Chilopoda . . 3

Myriapoda-Diplopoda . . . 34

myrmekurum (Strong.) . . 83

$\mathbf{N}$

Nannophilus . . . . . 6

Nanostreptus . . . . 86

nattereri (Leptod.) . . . 70

nattereri (Polyd.) . . . 70

27 nattereri (Rhinocr.) . . 37

41 nattereri (Spirob.) . . . 37

27 nesuphila (Scolop.). . . 27

21 Newportia . . . . 9

7 newportii (Scolop.). . . . 27

37 nigracans (Iul.) . . . . . 42

51 nigracans (Sirob.) . . . 42

51 nigrovittata (Scut.). . . . 34

85 vitida (Scolop.) . . . . 17

41 nitidior. (Spirostr. subseri-

41 ceus) . . . . . 55

42 nitidum (Strong.) . . . . 83

31 nodulipes (Rhinocr.) . . . 84

11 Notiphilides . . . . . 4

11 nudipes (Leptod.) . . . 71

51 obscura (Scolop.) . . . . 7

8.: olsecurne (Cormoc.). . . . 7

(.).) obscurus (Spirob.) . . . 42 


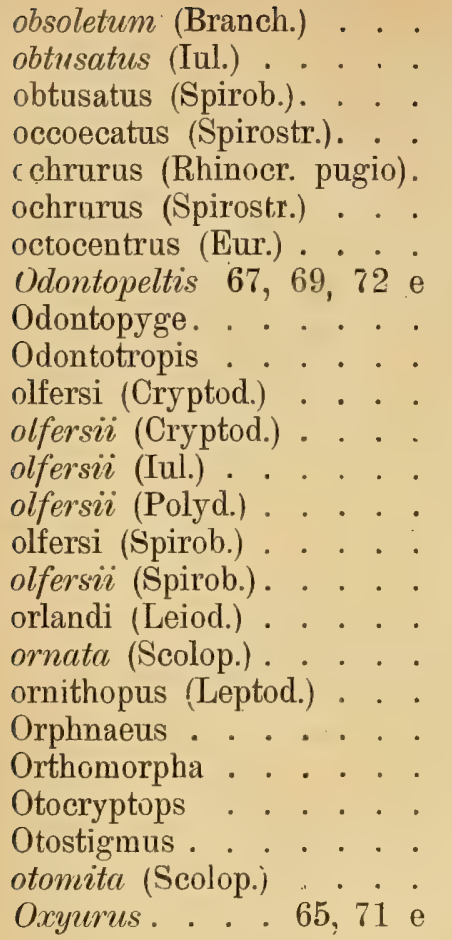

\section{$\mathbf{P}$}

pallidus (Leptod.) . . . pallidus (Ox.). papillaris (Spirostr.) . . Paradesmus. parae (Priod.). paraensis (Rhinocr.) . . paraensis (Spirob.). paraguayense. (Strong.) . paraguayensis (Heterop.) . paraguayensis (Odontopyge) parallelus (Brachyur.) . . parallelus (Leptod.). Paraspirobolus parmatus (Leptod). parmatus (Ox.) parmatus (Polyd.). parva (Scolop.)
14 parvidens (Scolop.). . . . 27

42 parvula (Pseud.). . . . . 85

42 patruelis (Spirostr.). . . . 52

51 paulista (Pseud.). . . . . 57

38 paulista (Schend.) . . . 6

51 paulistus (Leptod.). . . . 71

61 paulistus (Parasp.). . . . 35

86 pella (Scolop.). . . . . 23

85 perfidus (Spirostr.) . . . . 52

74 perlucens (Spirostr.) . . . 52

60 perproximus (Diap. ameri-

60 canus). . . . . 85

43 petropolites (Leptod.) . . . 71

60 phranus (Spirob.) . . . . 44

43 picturata (Scolop.). . . . 23

43 pileolus (Cryptod.) . . . . 60

86 pilosella (Scolop.) . . . . 23

27 pinnatus (Iul.) . . . . 79

70 pinnatus (Polyd.). . . . 79

5 Pithopus . . . 14,31 e 32

74 placeae (Scolop.). . . . . 27

10 planiceps (Scolop.). . . . ¿27

12 planipes (Scolop.) . . . . 23

31 platypoides (Scolop.) . . . 23

74 platypus (Scolop.) . . . . 23 Platyrrhacus . . . . 77

plumbeolata (Scolop.). . . 27

Plusioporus. . . . . . 85

71 poeyi (Parad.). . . . . 75

71 poeyi (Strong.) . . . . . 75

51 politulus (Cryptod.) • • . 60

75 Polydesmidae. . . 60 e 86

81 Polydesmoidea. . . . . 60

38 Polydesmus. . . . . . . 77

38 Polyzonidae. . . . . . 34

84 porphyrotaenia (Scolop.) . 23

86 praelongus (Spirob.) . . . 53

85 prasina (Scolop.) . . . 17

86 prasinipes (Scolop.) . . . 18

86 princeps (Spirostr.). . . . 53

35 Priodesmus. . . . . . 81

71 pseudofuscipes (Spirostr.) . 53

71 Pseudoleptodesmus. . . . 72

71 Pseudonannolene. . . . 56

31 Pseudonannolenidae. 56 e 85 
pugio (Rhinocr.). . . . puchra (Scolop.). . . . punctatus (Otocr.) .... . 12 puncticeps (Scolop.) . 7 e punctiscuta (Scolop.). . punctidens (Scolop.). . . punctidives (Spirob.) . . punctiplenus (Spirob.) . punctiporus (Arch.) . . punctiventris (Scolop.) . . punctulatus (Spirostr.) . . pusillus (Cryptod.) . pustulatum (Strong.) . pygomolas (Cormoc.).

\section{$\mathbf{R}$}

Rachidomorpha 63, 64, 66, $72,73 \mathrm{e}$. rarispina (Scolop.) Rhacophorus . 60, 66 e Rhinocricus. Rhoda. Rhombocephalus. Rhysida richardsonii (Scolop.). . . rosascens (Leptod.). . . . rosascens (Polyd.) . . . . rosascens (Rachid.). rosasceus (Polyd.) . rotundanus (Spirostr.)... . rubescens (Leptod.). . . . rubescens (Polyd.). rufipes (Platyrr.). rugosus (Spirob.) rugulosus (Polyd.) .

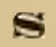

scabricauda (Branchiotr.) . sagrae (Scolop.) . saltatoria (Scolop.). santae-luciae (Spirob.) sanctus (Arch.) Sandalodesmus
38 . sandwchiana (Scolop.) . . 27

27 scaber (Platyrr.). . . . 77

12 scaber (Polyd.) . . . . . 77

17 scabricauda (Branch.). . . 13

17 scabricauda (Otost.) . . 13

30 Scaphiostreptus . 47, 49 e 53

44 scapulatus (Katant.) . . . 62

44 Schendyla . . . . . . . 6

45 Scolopendra. . . . . 15

31 Scolopendridae . . . . . 6

52 Scolopendrides . . . 9 e 10

60 Scolopendropsis . . . . . 31

84 Scolopocryptops . . . . . 33

7 scopoliana (Scolop.) . . . 23

Scutigera. . . . . . 34

Scutigeridae. . . . . 34 sebae (Iul.) . . . . . 50 sebae (Spirostr.) . . . . 50

81 sebastianus (Pseud. longi27 cornis). . . . . 57

70 sebastianus (Spirostr.). . . 53

35 semicinctus (Spirostr.) . . 54

32 septemspinosa (Scnlop.) . . 27

29 sericiventris (Rhinocr.) . . 38

14 serrulatus (Iul.) . . . . . 80

23 serrulatus (Polyd.) . . . . 80

72 setiger (Spirostr.) . . . . 54

72 sexspinosa (Crypt.). . . . 11

72 sexspinosa (Scolop.) . . . 28

72 sexspinosa (Scolop. subspi-

53 nipes) . . . . . . 29

72 srxspinosa (Scolopocr.) . . 11

72 sexspinosus (Crypt.) . . . 11

77 sexspinosus (Otocr.) . . . 11

44 sexspinosus (Scolopocr.) . 11

80 silhetensis (Scolop.) . . . 28 simillimus (Leptod.) . . . 72

Siphonotus . . . . . . 34

slateri (Euryl.) . . . . . 24

13) smaragdinus (Rhomb.) . . 29

16 spilonotum (Strong.). . . 84

23 spinicauda (Scolopocr.). . 12

44 Spirobolellus . . . . 39

45 Spirobolidae . . . . 85 o 8.1

8 (i) Spirobolus . . . . . . 39 


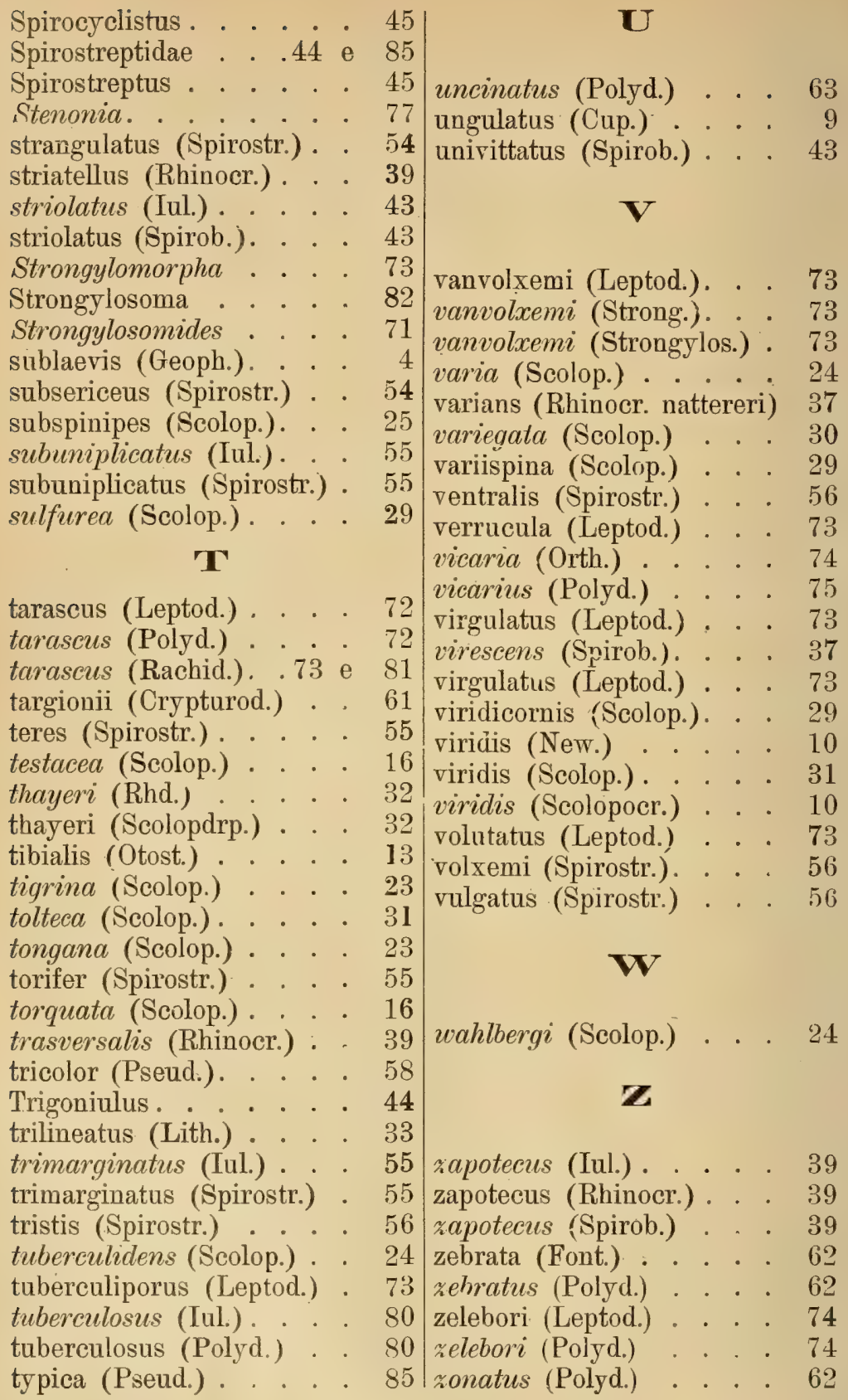




\section{CATALOGOS}

DA

FHUX\% BR\%ZIIFIRH EDITADOS PELO

Museu Faulista S. PAULO-BRAZIL

Vol III
AS COCOIDAS DO BEAZII ADOLPH HEMPEL

Entomologista do Instituto Agronomico de Campinas, Est. de S. Paulo

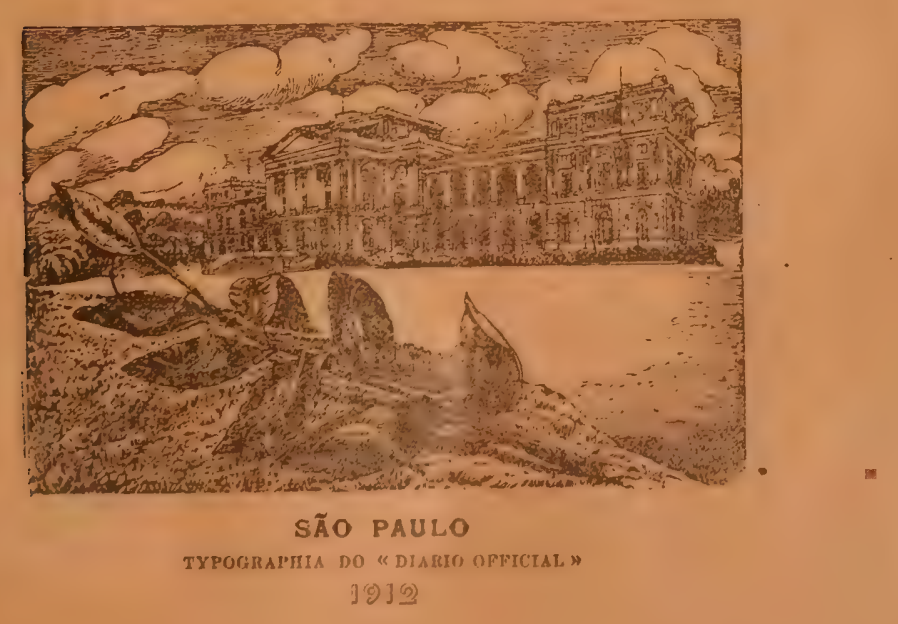





\section{CATALOGOS}

DA

\section{FHUY\% BR\%ZILEIRH}

EDITADOS PELO

Museu Paulista

S. PAULO-BRAZIL

\section{Vol. III}

\section{AS COCOIDASDO BEAZIL}

POR

\section{ADOLPH HEMPEL}

Entomologista do Instituto Agronomico de Campinas, Est. de S. Paulo

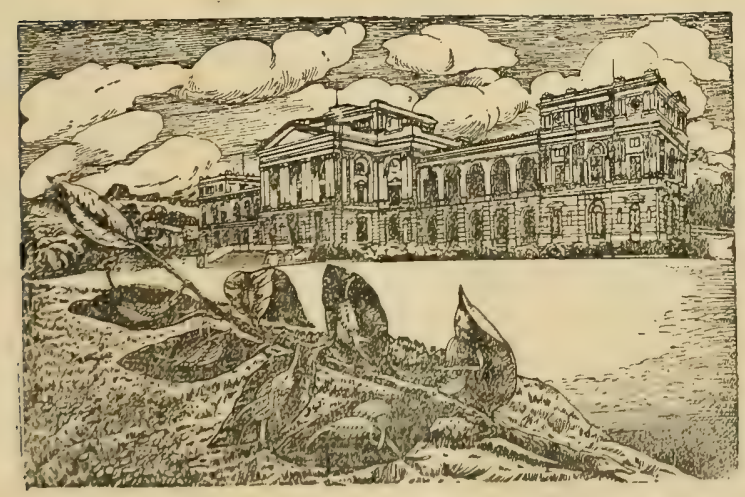

SĀO PAULO

TYPOGRAPRIA DO "DIARIO OFFICTAL" 

Dando ao prélo o Catalogo das Coccidas do Brazil, elaborado pelo Snr. Adolph Hempel, Phytopathologista do Instituto Agronomico de Campinas, Estado de S. Paulo, sinto natural satisfacção pelo bom exito a que conduziram as pesquizas iniciadas no Museu Paulista sobre estes piolhos vegetaes. No primeiro estudo que publiquei sobre o assumpto, (Rev. Mus. Paul., II) consegui reunir na lista das Coccidas do Brazil apenas 2 I especies. Foi a doença das jaboticabeiras que me havia levado a taes investigações e, entrando por esta epoca o Snr. A. Hempel para o quadro do pessoal do Museu, como entomologo, incumbi-o da continuação destes estudos, dos quaes resultou a bella monographia das Coccidas publicadas na Revista deste Museu, vol. IV. Com este alentado trabalho o Snr. Hempel inscreveu o seu nome na lista dos entomologos competentes e geralmente apreciados da actualidade. Desde a publicação daquella monographia que deu a descripção detalhada de I3 I especies de Coccidas, tanto o seu autor quanto o pessoal do Museu não cançaram no proseguimento destas investigações. De valor especial para o trabalho do Museu foi o laboratorio de que o mesmo dispõe no Alto da Serra, sob a denominação de Estação Biologica, e diversas das especies alli descobertas, sendo novas para a sciencia, foram descriptas pelo Snr. Hempel, em parte como annexo a este catalogo. Por todos estes esforços combinados o autor enumera como especies conhecidas do Brazil 16 ; Coccidas.

A direcção do Museu Paulista, que se ufana de ter introduzido no Brazil o estudo da entomologia economica sobre base scientifica, agradece ao Snr. A. Hempel a elaboração desinteressada do presente catalogo e ao seu illustre chefe, Snr. Arthaud-Berthet, Director do Instituto 
Agronomico, a amavel collegialidade com que sempre auxiliou as intenções desta repartição. Entendemos que o conhecimento destes terriveis inimigos da agricultura e silvicultura é uma medida indispensavel para sua destruição e que neste sentido, publicações desta ordem terão, além de seu valor scientifico, tambem a mais alta importancia economica.

São Paulo, 3i de Março de 19i2.

DR. HERMANn von IHERING. 


\section{INDICE}

I Introducção.

II Relação das Publicações (abreviaturas).

III Bibliographia das Coccidas Brazileiras.

VI Literatura Geral sobre as Coccidas.

$\mathrm{V}$ Indice Systematico.

VI Enumeração das especies.

VII Descripção das especies novas.

VIII Indice Alphabetico. 



\section{As Coccidas do Brazil}

\section{INTRODUCÇÃO}

Doze annos são passados desde que foi publicado o meu pequeno trabalho sobre «as Coccidas Brazileiras» no qual foram descriptas 131 especies desta familia de insectos nocivos. Durante este tempo foram encontradas outras especies, algumas novas para a sciencia, outras já conhecidas mas cuja presença no Brazil foi apenas agora constatada, até que, actualmente, são conhecidas, como fazendo parte da fauna brazileira, nada menos de 165 especies e variedades.

Todos estes insectos pertencem á familia Coccidae da ordem Hemiptera, e, por seu caracter parasitico, têm elles uma certa importancia economica em todos os paizes agricultores.

Convidado pelo dr. H. v. Ihering, digno Director do Museu Paulista para collaborar na publicação dos catalogos da Fauna Brazileira, preparando o manuscripto para o catalogo das Coccidas do Brazil, acceitei o honroso convite, porque julguei opportuno reunir, em urn só volume, os novos dados sobre esta familia de insectos, accumulados em doze annos de estudos. A falta de monographias, compendios e catalogos sobre a fauna brazileira é sobremodo sensivel entre nós, e muitos novos estudantes e investigadores desanimam logo nas suas primeiras pesquizas, pela ausencia da literatura competente; assim julgo que este catalogo pode servir para estimular o estudo desta familia de insectos.

O catalogo dá o nome scientifico de cada especie, o nome do auctor, indica onde foi primeiramente descripta e dá a sua destribuiçăo geographica bern como o nome das plantas da qual é parasita a especie em questão. Contem tambem referencias á literatura mais importante e recente, tanto da de natureza geral, como áquella que trata só das especies brazileiras; com referencias ás especies indigenas essa bibliographia é completa. 
Julguei inutil dar a synonymia completa das especies, pois isto seria repetir o trabalho já existente no Catalogo das Coccidas do Mundo, publicada pela Snra. d. Maria E. Fernald, - qual contem uma synonymia completa de todas as especies conhecidas até 1903.

Campinas, 2 de Junho de 1911.

\section{RELAÇÃO DAS PUBLIICAÇÕES}

\section{(Explicação das abreviaturas)}

Am. Nat. The American Naturalist. Philadelphia.

Ann. Mag. N. H. Annales and Magazine of Natural History. London.

Ann. Soc. Ent. Fr. Annales de la Societé Entomologique de France. Paris. Paulo.

Bol. Agri. Boletim da Agricultura do Estado de S.

Bol. do 1. A. Boletim do Instituto Agronomico do Estado de S. Paulo. S. Paulo.

Boll. Mus. Torino. Bolletino dei Musei di Zool. ed Anat. comparata della R. Universitá di Torino.

Bull. Soc. Ent. Fr. Bulletin de la Societé Entomologique de France. Paris.

Can. Ent. The Canadian Entomologist. London. Can.

Cat. Br. Mus. Hom. Catalogue of Insects in the British Museum, Homoptera. (Walker). London.

Deutsch. Ent. Zeit. Deutsche Entomologische Zeitschrift. Berlin.

Ent. Mon. Mag. The Entomologist's Monthly Magazine. London.

Ent. News. Entomological News. Philadelphia Pa.

Essai Hist. Nat. des Oranges. Essai sur l'Histoire Naturelle des Oranges. (Risso). Paris, 1818.

Gard. Chron. The Gardener's Chronicle. London.

In. Agr. Insectologie Agricole Paris, 1867 a 1870.

Ind. Mus. N. Indian Museum Notes. Bombay.

Jahrb. Ham. Wiss. Anst. Jahrbuch der Hamburgischen Wissenschaftlichen Anstalten. 
Journ. Quek, Micr. Club. Journal of the Quekett Microscopical Club. London.

Journ. Ins. Jam. Journal of the Institute of Jamaica. Kingston.

Mem. d'Hist. Nat. Sc. Mémoires d'Histoire Naturelle, Académie de Scierce. Paris.

N. Z. Trans. Transactions of the New Zealand Institute. Wellington.

Naturg. C. bromelice. Naturgeschichte des Coccus bromelice. (Kerner). Stuttgart, 1778.

R. Dep. Ent. Corn. Univ. Report of the Department of Entomology of Cornell University. Ithaca, 1883.

Rep. U. S. Dept. Ag. Report of the United States Department of Agriculture. Washington.

Rev. Revista do Museu Paulista. S. Paulo.

Rev. Agr. Revista Agricola. S. Paulo.

Rev. Chil. Hist. Nat. Kevista Chilena de Historia Natural. Valparaiso.

Schäd. Gart. In. Naturgeschichte der Schädlichen Garten Insecten (Bouché). Berlin. 1833.

Spic. Zool. Spicilegia Zoologica. (Gray). London (1829).

Stett. Ent. Zeit. Stettiner Entomologische Zeitung. Berlin.

Studii sul. Cocc. Studii sulle cocciniglie. (Targioni-Tozzetti). Milano, 1867.

Syst. Nat. Syst. Natural. (Linnaeus), editio.

Targ. Toz. Cat. Coccidarum Catalogus. (Targioni-Tozzetti). Milano, 1868.

The Ent. The Entomologist. London.

Tr. Ent. Soc. Lon. Transactions of the Entomological Society of London. London.

Zeit. $f$. W. In. Zeitschrift für Wissenschaftliche IUsektenbiologie. Berlin.

\section{BIBLIOGRAPHIA DAS COCCIDAS BRAZILEIRAS}

Campos Novaes, José de, 1897. - «Uma Doença das Jaboticabeiras». Revista Brazileira, Tomo XI, Fasciculo 626 p. p. 113-118, Julho de 1897.

Campos Novaes, José de, 1899. - «A Molestia das Jaboticabeiras». Kevista Brazileira, Tomo XVII, Fasciculo 86, p. p. 227-244, 1899. 
Cockerell, T. D. A., 1893. - «Notes on Lecanium with a List of the West Indian Species». Transactions of the American Entomological Society, p. p. 49-56, Philadelphia, April, 1893.

Cockerell, T. D. A., 1893. - «A New Subspecies of Ceroplastes from Mexico». Zoe. Vol. IV, No. 1, p. p. 104106, San Francisco, Cal., April, 1893.

Cockerell, T. D. A., 1894. - «A New Wax-Scale found in Jamaica». Entomological News, p. 157, Philadelphia, 1894.

Cockerell, T. D. A., 1894. - "Coccidae or Scale Insects». V. Bulletin of the Botanical Dept. Jamaica, Vol. I, part 5, p. p. 69-73. Kingston, Jamaica, May, 1 1:94.

Cockerell, T. D. A., 1894. - «Description of New Coccidae». Entomological News, p. p. 203-204, Philadelphia, June, 1894.

Cockerell, T. D. A., 1894. - «The Distribution of Coccidae». Annals and Mag. of Natural History, Series 6, Vol. XIV, p. p. 76-80, London, July, 1894.

Cockerell, T. D. A., 1895. - «Goccidae or Scale Inects». VI. Bulletin of the Botanical Dept., Jamaica, Vol. II, part 1, p. p. 5-8, Kingston, Jamaica, Jan. 1895.

Cockerell, T. D. A., 1895 - «Two New Species of Lecanium from Brazil. The American Naturalist, Vol. 28, p. p. 178-175. Philadelphia, Feb. 1895.

Cockerell, T. D. A., 1895. - «Thow New Species of Coccidae. The Entomologist, p. p. 100-101, London, England, 1895.

Cockerell, T. D. A., 1895. - «Goccidae or Scale Insects. VII». Bulletin of the Botanical Dept., Jamaica, Vol. I, part. 5, p. p. 100-102. Kingston, Jamaica, May, 1895.

Cockerell, T. D. A., 1895. - «On the Sub-globular Species of Lecanium». The Canadian Entomologist, Vol. XXVII, p. p. 201-204, London, Ontario, August, 1895.

Cockerell, T. D. A., 1897. - "Notes on the Coccidae, a Family of Homoptera, with a table of the Species hitherto observed in Brazil». Revista do Museu Paulista, Vol. II, p. p. 65-72, S. Paulo, 1897.

Cockerell, T. D. A., 1897. - «Further Notes on Coccidae from Brazil». Revista do Museu Paulista. Vol. II, p. p. 383-384, S. Paulo, 1897.

Cockerell, T. D. A., 1898. - «Goccidae or Scale Insects». XII. Bulletin of the Botanical Dept., Jamaica, Vol. V, part 2, p. p. 40-44. Kingston, Jamaica, Feb., 1898. 
Cockerell, T. D. A., 1898. - «Three New Coccidae of the Sub-family Diaspinae». Psyche, Vol. VIII, p. p. 201-202. Gambridge, Mass., U. S. A., April, 1898.

Cockerell, T. D. A., 1899. - «Some New Goccidae callected at Campinas, Brazil, by Dr. F. Noack». Revista do Museu Paulista, Vol. III, p. p. 41-42, S. Paulo, 1898.

Cockerell, T. D. A., 1898. - «Mais algumas Coccidas colligidas pelo Dr. F. Noack». Revista do Museu Paulista, Vol. III, p. p. 501-503, S. Paulo 1898.

Cockerell, T. D. A., 1898. - «Some New Coccidae of the Sub-family Lecaniinae». The Entomologist. p. p. 130-132. London, England, Jan., 1898.

Cockerell, T. D. A., 1899. - «Two New Genera of Lecanine \& Coccine Coccidae». The Entomologist, p. p. 12-13, London, England, Jan. 1899.

Cockerell, T. D. A., 1899. - «Thre New Coccidae from Brazil». The Canadian Entomologist, Vol. XXXI, No. 2, p. p. 43-45. London, Ontario, Feb., 1899.

Cockerell, T. D. A., and P. J. Parrott, 1899. - « «ontributions to the knowledge of the Goccidae». The Industrialist, p. p. 276-284, May, 1899.

Cockerell, T. D. A. 19u0. - Notas sobre Coccidas Brazileiras. Revista do Museu Paulista, Vol. IV, p.p. 363-364, S. Paulo, 1900.

Cockerell, T. D. A. 1902.-Some Brazilian Goccidae. Annals and Magazine of Natiral History, Ser. 7, vol. IX., p.p. 451-456. London, England, June, 1902.

Goeldi, Dr. E. A., $1 \times 86$. -Beitraege zur Kenntniss der kleinen und kleinsten Gliederthierwelt Brasiliens. Mittheilungen der Schweiz. Entomol. Gesellschaft. Bd. 7, p.p. 283-355, $188 \mathrm{x}$.

Goeldi, Dr. E. A., 1886.-Apontamentos de Zoologia Agricola e Horticultura. Jornal do Agricultor. Tomo XIV, n. 346, p.p. 1:0-111, Rio de Janeiro, Fev. de 1886.

Goeldi, Dr. E, A., 18.9.9. - «Epeiroides bahiensis Keyserling. Eine Daemmerungs Kreuzspinne Brasiliens. Zoologische Jahrbücher, Band XII, p.p. 161-169, Jena, 1899.

Hempel, Adolph, 1898. - Notas sobre Capulinia Jaboticabce Ihering. Revista do Museu Paulista, Vol. III, p.p. 51-62. S. Paulo, 1898 ,

Hempel, Adolph, 1899. Two new Coccidae of the Subfamily Lecaniincue. The Canadian Entomologist, Vol. XXXI, n. 6, p.p. 131-133, London, Ontario, June 1899. 
Hempel, Adolph, 1900.-Descriptions of Three New Species of Coccidae from Brazil. The Canadian Entomologist, Vol. XXXII, n. 1, p.p. 3-7, London, Ontario, Jan., 1900.

Hempel, Adolph, 1900. - As Coccidas Brazileiras. Revista do Museu Paulista, Vol. IV, p.p. 365-537. S. Paulo, 1900.

Hempel, Adolph, 1900. Descriptions of Brazilian Coccidae. Annals and Magazine of Natural History, Ser. 7, Vol. VI, and following, London, England, 1900.

Hempel, Adolph, 1901.-A Preliminary Report on some new Brazilian Hemiptera. Annals and Magazine of Natural History, Ser. 7, Vol. VIII, p.p 383-391, London, England, Nov. 1901.

Hempel, Adolph. 1902.-Notas sobre alguns Insectos Nocivos. Boletim da Agricultura, 3 serie, p.p. 237-255, S. Paulo, Maio de 190:

Hempel, Adolph, 1904.-Resultado do exame de diversas Gollecções de Coccidas enviadas ao Instituto Agronomico pelo Snr. Carlos Moreira, do Museu Nacional, Rio de Janeiro. Boletim da Agricultura, 5 serie, p.p. 311-323, S. Paulo, Julho de 1904.

Hempel, Adolph, 1910.-Descripção de um novo Genero e uma nova Especie de Coccidas. Revista do Museu Paulista. Vol. VIII p.p. ¿2-53, S. Paulo, Abril de 1910.

lhering, Nr. H. v., 1597.-Os piolhos vegetaes (Phytophthires) do Brazil. Revista do Museu Paulista, Vol. II, p.p. 385-420. S. Paulo, 1897.

Ihering, Dr. H. v., 1898. - A doença das jaboticabeiras. Revista Agricola, Vol. IV, n, 35, p.p. 185-189. S. Paulo, Junho de 1 4 y, e tambem Revista do Museu Paulista, Vol. III, p.p. 45-49, S. Paulo, 1898.

Ihering, Dr. H. v., 1899.-Prejuizos causados em S. Paulo ás larangeiras por piolhos vegetaes. Revista Agricola, Vol. 5, n. 44, p.p. 89-91, S. Paulo, Março de 1899.

Ihering, Dr. H. थ., 1 $\times 99$. - Notas sobre as especies de Aspidiotus. Revista Agricola, Vol. n. 54, p.p. 13-18, S. Paulo, 1899.

Lindinger, Dr. Leonhard, 1910.-Beitraege zur Kenntnis der Schildläuse und ihrer Verbreitung. II. Zeitschift für Wissenschaftliche Insekteniologie, Band VII, Heft 1, p.p. 9-12, Berlin, Jan. de 1911.

Moreira, Carlos, 1899.-Gontra os inimigos. A Lavoura V. 2. ${ }^{a}$ serie, p.p. 140-144. 1899. 
Silvestri, F., 1901.-Nota preliminare sur Termitidi sudamericani. Boll. Mus. Torino. XVI, n. 395, p. 41901.

d'Jtra, Dr. G. R. P. 1899.-A fumagina ou morphéa das Larangeiras. Boletim do Instituto Agronomico do Estado de S. Paulo, Vol. X, ns. 9 e 10, pp. 6.14-610, Campinas, Set. e Out. de 1899.

\section{LITERATURA GERAL SOBRE AS COCCIDAS}

Cockerell, T. D. A., 189' - «A Check-List of the Coccidae of the Neotropical Region». Journal of the Trinidad Field Naturalists Glub. Vol. 1, p. p. 311-312. Port of Spain, Trinidad, 1894.

Cockerell, T. D. A., 1895. - "Notes on the Geographical Distribution of Scale Insects». Proceedings of the U. S. National Museum, Vol. XVII, p. p. 615-625, Washington, 1895 ,

Cockerell, T. D. A., 1896. - «A Check-List of the Coccidae». Bulletin of the Illinois State Laboratory of Natural History, Vol. IV, Article XI, p. p. 318-339, Urbana, Illinois, July, 1896.

Cockerell, T. D. A., 1997. - «The Food Plants of Scale Insects» (Coccidae). Proceedings of the U. S. National Museum, Vol. XIX, p. p. 725-786, Washington, 1897.

Cockerell, T. D. A., 1897. - «The San José Scale and its Nearest Allies». U. S. Dept. of Agriculture. Division of Entomology. Technical series, No. 6, Washington, 1897.

Cockerell, T. D. A., 1899. - «First supplement to CheckList of the Coccidae». Bulletin of the Illnois State Laboratory of Natural History, Vol. V, Article VII, p. p. 389-398, Urbana, Illinois, Jan, 1899.

Cockerell, T. D. A., \& P. J. Parrott, 1899. - Biologia Centrali Americana, Rhynchota, Homoptera, December, 1899.

Comstock, J. H., 1881. - "Report of the Entornologist of the U. S. Dept. of Agriculture for the year 1880, Washington, 1881 .

Comstock, J. H., 1883.- «Second Report of the Cornell University Experiment Station». Ithaca, N. Y, 1883. 
Fernald, Mrs. Maria E., 1903. - «A Catalogue of the Coccidae of the World». Hatch Experiment Station of the Massachusetts Agricultural College, Bulletin N. 88, Amherst, Mass., 1903.

Green, E. Ernest, 1896-1904.— «The Coccidae of Ceylon». Part I, London, 1896. Part II, London, 1899. Part III, London, 1904.

Maxwell-Lefroy, H., 1902. - «Scale Insects of the West Indies». The West Indian Bulletin, Vol. III, N. 3, p. p. 240-270, 1902.

Nevostead, R., 1900. - «The Injurious Scale Insects and Mealy Bugs of the British Isles». Journal of the Royal Horticultural Society, Vol. XXIII, Part 3, p. p. 1-44, London, England, 1900.

Newstead, R., 1901-1903.- "A Monograph of the Cioccidae of the British Isles». Ray Society, London, England, 1901-1903.

Sanders, $J$. G., 1406. - «Catalogue of recently described Coccidae». U. S. Dept. of Agriculture, Bureau of Entomology. Technical Series N. 12, Part I, Washington, June, 1906.

Sanders, J. G., 1909. - «Catalogue of recently described Coccidae, II». U. S. Dept. of Agriculture, Bureau of Entomology, Technical Series, N. 16, Part III, Washington, December, 1909.

Signoret, V., 1868-1876.- «Essai sur les cochenilles». Annales de la Societé Entomologique de France. Paris, $1868-1876$.

Targioni-Tozzetti, 186\%. - «Studi sulle Cocciniglie». 1867.

Targioni-Tozzetti, 1819._Coccidarum Catalogus. 1869.

Tambem se acham muitos artigos sobre Coccidas nas seguintes publicações :

Reports and Bulletins of the U.S. Department of Agriculture, Washington, D. G., U. S. A.

Publicações das diversas Estações Experimentaes na America do Norte.

Transactions and Proceedings of the New Zealand Institute. Wellington, New Zealand.

The Entomologist's Monthly Magazine. London, England.

The Canadian Entomologist. London, Ontario.

The Entomological News. Philadelphia, Pa., U. S. A.

Psyche, Gambridge, Mass., U. S. A.

Bulletin e Annales de la Societé Entomologique de France, Paris. 


\section{INDICE SYSTEMATICO}

Ciasse Hexapoda

\section{Ordem HEMIPTERA}

SUB-ORDEM HOMOPTERA

FAM LIA COCCIDAE

Sub-familia Monophlebinae

Stigmacoccus Hempel. Palaeococcus Cockerell Icerya Signoret

Sub-familia Ortheziinae

Orthezia Bosc.

Sub-familia Conchaspinae

Conchaspis Cockerell

Sub-familia Dactylopinae

Asterolecanium Targ.

Lecaniodiaspis Targ.

Solenococcus Cockerell

Eriococcus Targ.

Tectococcus Hempel.

Carpochloroides Cockerell

Apiococcus Hempel

Capulinia Signoret

Cryptokermes Hempel

Phenococcus Cockerell
Lachnodiella Hempel

Pseudococcus Westwood

Erium Crawford

Ripersia Signoret

Chaetococcus Maskell

Termitococcus Silvestri

Tachardia Blanchard

Sub-familia Coccinae

Pulvinaria Targ.

Protopulvinaria Cockerell

Pulvinella Hempel

Tectopulvinaria Hempel

Mallocoscus Maskell

Lichtensia Signoret

Ceroplastes Gray

Vinsonia Signoret

Alichtensia Cockerell

Edwailia Hempel

Platinglisia Cockerell

Pseudokermes Cockerell

Encalymnatus Cockerell

Stictolecanium Cockerell

Coccus Linnaeus

Mesolecanium Cockerell

Neolecanium Parr

Akermes Cockerell

Eulecanium Cockerell

Paralecanium Cockerell

Saissetia Deplanches

Lecanium 
Sub-familia Diaspinae.

Chionaspis Signoret

Diaspis Costa

Aulacaspis Cockerell

Hemichionaspis Cockerell

Pinnaspis Cockerell

Fiorinia Targ.

Aspidiotus Bouché

Morganella Cockerell

Pseudaonidia Cockerell

Selenaspidus Cockerell Chrysomphalus Ashmead

Pseudischnaspis Hempel

Odonaspis Leonardi

Pseudoparlatoria Cockerell

Diaspidistis Hempel

Gymnaspis Newstead

Lepidosaphes Shimer

Ischnaspis Douglas

Parlatoria Targ.

Descripęão de 5 especies novas : Icerya genistae, Ripersia taquarae, Saissetia lucida, Puivinaria ornata, Pseudoparlatoria argentata. 


\section{ENUMERAÇÃO DAS ESPECIES BRAZILEIRAS}

Da familia das Coccidas, insectos, que pertencem á ordem dus Hemipteros.

Sub-ordem dos HOMOPTEROS

Familia COCCID $A$

SUB-FAMLIL MONOPHLEBIN Æ

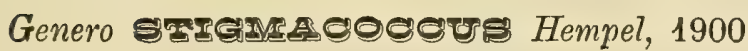

1. Stigmacoceus asper Hempel.

Stigmacoccus asper Hempel, Rev. IV, p. 400, 1900.

Hab. Estado de S. Paulo, Rio Mogy-Guassú, perto de Pirassununga.

Mus. Paul. : N. 389, Rio Mogy-Guassú (Est. de S. Paulo), Hempel leg. Typo.

Em Inga sp. Agrupados no lado inferior dos galhos e ramos, e acompanhados de uma formiga do genero Camponotus.

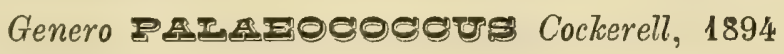

\section{Palaeococeus braziliensis (Walker).}

Monophlebus braziliensis Walker, Cat. Br. Mus. Hom., p. 1089, 1852 .

Hab. America do Sul.

3. Palaeococeus hompeli (Cockerell).

Icerya hempeii Ckll. Can. Ent. XXXI, p. 43. 1899.

Crypticerya hempeli Hempel, Rev. IV, p. 376, 1900.

Hab. Estado de S. Paulo, Gampinas, Baurú.

Mus. Paul. N. 258. Campinas (Est. de S. Paulo) Dr. Noack leg. Co-typo.

Em galhos de Mimosa sp. 


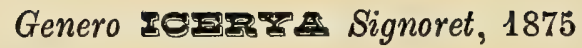

\section{Icerya braziliensis Hempel.}

Icerya braziliensis Hempel. Rev, IV, p. 370, 1900. Hab. Estado de S. Paulo, Ypiranga, S. Paulo, Iguape. Mus. Paul. N. 324 S. Paulo (cidade) Hempel leg. Typo; N. 15633, idem, F. Lex leg.; N. 16235, Ypiranga, S. Paulo. Em Liriodendron tulipifera L., Laurus camphora L., Codiaeurn sp., Ficus sp. e roseira.

\section{Icorya gonistae $n . s p \cdot\left(^{*}\right)$}

Hab. Estado de S. Paulo. Campinas.

Mus. Paul. N. 16:93, Campinas (Est. de S. Paulo,) Hempel leg. Co-typo.

Em Genista scoparia Lam., Lespedeza striata H. e A., e morango, Fragaria sp.

\section{Icerya schrottkyi Hempel.}

lcerya schrottkyi Hempel, Rev. IV, p. 373, 1900.

Hab. Estado de S. Paulo, Jundiahy.

Mus. Paul. N, 391, Jundiahy, Est. de S. Paulo, Schrottky leg. ; N. 10710, S. Paulo, cidade.

Em uma arvore sylvestre.

\section{SUB-FAMILIA ORTHEZIIN $尹$}

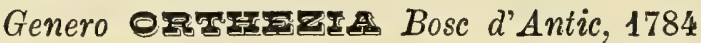

\section{Orthezia insignis Douglas.} 1887.

Orthezia insignis Douglas, Jn. Quek, Mic. Club, p. 169,

Orthezia insignis Hempel, Rev. IV, p. 376, 1900.

Hab. Estado de S. Paulo, Campinas, Estado de Minas Geraes, Aguas Virtuosas, America do Norte, Mexico, Jamaica, Trinidade, Inglaterra, Africa do Sul, Ceylão, Mauricia e China.

Mus. Paul. N. 393, Aguas Virtuosas (Est. Minas Geraes) Alvaro da Silveira leg.

Em Coleus; Lantana, Ipomoea, Thunbergia, Verbena, Strobilanthus, Achillea, Salvia, Cuphea, Capsicum, Ageratum,

(*) Veja-se a descrip̧̨ão no fim deste catalogo. 
Vernonia, Gardenia, Chrysanthemum, Lonicera, Citrus, chá, morango, tomate e outras plantas cultivadas.

\section{Orthezia praelonga Douglas.}

p. $246,1891$.

Orthezic praelonga Douglas, Ent. Mon. Mag. XXVII,

Orthezia praelonga Hempel, Rev. IV, p. 377, 1900.

Hab. Estado de S. Paulo, Ýpiranga, Estado do Pará, Jamaica, Trinidade e Guiana Ingleza. nidade.

Mus. Paul. N. 15̌634, Ypiranga, S. Paulo, N. 193, Tri-

Em Hyptis, Citrus, Capsicum, Groton, Sanchezia.

\section{Sub-pamilia CONCHASHIN ÆE}

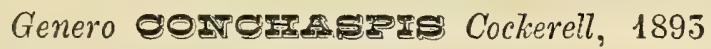

\section{Conchaspis fuminensis Hempei.} 1904.

Conchaspis fluminensis Hempel, Bol. Agri, V, p. 312, Hab. Rio de Janeiro.

Em uma planta sylvestre.

\section{SUB-FAMLIL DACTYLOPIN $\approx$}

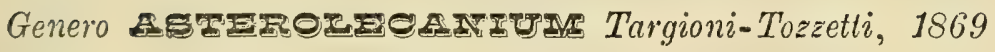

10. Asterolecanium lbambusae Boisduva?.

Asterolecanium bambusce Boisduval, In. Agr., 1869.

Asterolecanium bambusce Hempel, Rev. IV, p. 409, 1900

Hab. Estado de S. Paulo. Campinas, Mexico, Indias Occidentres, Ceylão, Algeria, Mauricia.

Ifus. Paul. 15.ô43, Campinas, S. Paulo, Dr. F. Noack leg.; N. 215, idem, idem.

Em bambú cultivado.

11. Asteroloranium limeare Lindinger.

Asterolecanium lineare Lin., Jəhrb. Ham. wiss, Anst., XXVI, 1908.

Hab. Brazil.

Em Cocos nucifera L. 


\section{Asterolecanium miliaris Boisduvat.}

Asterolecanium miliaris Boisduval, In. Agr., 1869. 1900.

Asterolecanium miliaris Hempel, Rev. IV, pag. 409 ,

Hab. Estado de S. Paulo, Cubatão, S. Paulo, Cachoeira, Trinidade, Jamaica, Alegria, Mauricia.

Mus. Paul. N. 247 e 15.724 Cachoeira (Est. de S. Paulo, Hempel leg.; N. 347, S. Paulo (cidade), Hempel leg. Em bambúi cultivado.

\section{Asterolecamium pustulans (Cockerell).} 1/3, 1892. 1900.

Asterodiaspis pustulans Gkll., Journ., Inst. Jam., I, p. Asterolecanium pustulans Hempel, Rev. IV, p. 409,

Hab. Estado de S. Paulo, Campinas, Jamaica, Porto Rico, Antigua, Grenada, Mexico, Ameriea do Norte.

Mus. Paul. N. 2:35, Campinas (Est. de S. Paulo) Dr. F. Noack leg.; N. 12.915, Estação do Rio Grande (Est. do S. Paulo).

Em pecegueiros, macieiras, figueiras, mangueiras, guandú, amoreira, Anona, Hibiscus.

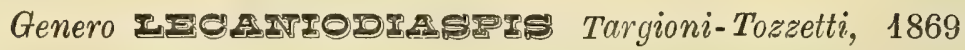

\section{Lecaniodiaspis rugosa Hempel.}

Lecaniodiaspis rugosus Hempel, Rev. IV, pag. 407, 1900. Hab. Estado de S. Paulo, Ypiranga. Tyро.

Mus. Paul. N. 275, Ypiranga, S. Paulo, Hempel leg.

Em ramos de uma arvore sylvestre.

\section{Genero Bex}

\section{Solonococeus baccharidis Hempel.} 1900.

Solenococcus baccharidis Hempel, Rev. IV, pag. 392,

Hab. Estado de S. Paulo, Ypiranga, S. Paulo.

Mus. Paul. N. 320, S. Paulo, Ypiranga, Typo; N. 16.236 e 15.636, idem, Hempel leg. 
Em galhos e tronco de Baccharis dracunculifolia D. C.

16. Solenococeus tuberculus Hempel.

Solenococcus tuberculus Hempel, Rev. IV, p. 390, 1900. Hab. Estado de S. Paulo, S. Paulo, Typo.

Mus. Paul. N. 15.645, S. Paulo (cidade) Hempel leg.

Em Baccharis sp.

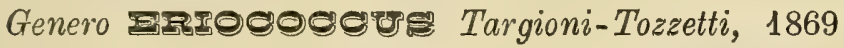

17. Eríococeus araucariae Maskell. 1878.

Eriococcus araucarice Maskell, N. Z. Trans., XI, p. 218,

Hab. Estado de S. Paulo, Campinas; Nova Zelandia, Ilhas Hawai, Ceylão, Africa do Sul, Mexico,

Mus. Paul. N. 16.294, Campinas, (Estado de S. Paulo), Hempel leg.

Em Araucaria excelsa.

18. Eriococeus brazilensis Cockerell.

Eriococcus braziliensis Ckll., Rev. IV, p. 363, 1900.

Eriococcus braziliensis Hempel, Rev. IV, p. 380, 1900. Hab. Estado de S. Paulo, Ypiranga.

Mus. Paul. N. 314, Ypiranga, S. Paulo, dr. H. v. Ihering, leg.; N. 14.428, idem.

Baccharis dracunculifolia D. C.

\section{Eriococeus perplexus Hempel.}

Eriococcus perplexus Hempel, Rev. IV, p. 381, 1900.

Hab. Estado de S. Paulo, Ypiranga, Estado de Minas Geraes, Bello Horizonte.

Mus. Paul. N. 299, Ypiranga, S. Paulo, Hempel, leg. Typo; N. 394, idem; N. 366, Belle Horizonte (Est. de Minas Geraes) Ol. de Assis leg.

Em Eugenia jaboticaba e Myrtacece.

Genero segrogogous Hempel, 1900

20. Tectococeus ovatus Hempel.

Tectococcus ovatus IIempel, Rev. IV, p. 406, 1900. 
Hab. Estado de S. Paulo, Ypiranga, S. Paulo.

Mus. Paul. N. 354, S. Paulo (cidade) Hempel. leg. Typo; N. 14.432, Ypiranga, S. Paulo.

Em folhas de Myrtacece.

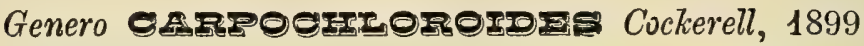

\section{Carpochloroides viridis Cockerell.} 1899.

Carpochloroides viridis Gkll., The Ent., XXXII, p. 12

Carpochloroides viridis Hempel, Rev. IV, p. 393, 1900.

Hab. Estado de S. Paulo, Ypiranga, Campinas.

Mus. Paul. Ns. 315 e 15.685. Ypiranga, S. Paulo; N. 16.303, Campinas (Est. de S. Paulo) Hempel leg.

Ém Eugenia sp., Myrtacece.

\section{Genero 로이이요 Hempel, 1900}

\section{Apiococcus asperatus Hempel.}

Apioccoccus asperatus Hempel, Rev. IV, p. 404, 1900. Hab. Estado de S. Paulo, Ypiranga.

Mus. Daul. N. 15.640, Ypiranga, S. Parlo, Hempel leg. Typo.

Em Myrtaceae.

\section{Apiococeuns globosus Hempel.}

Apiococcus globosus Hempel, Rev., IV, p. 405, 1900.

Hab. Estado de S. Paulo. S. Paulo. Typo.

Mus. Paul. N. 15.641, S. Paulo (cidade) Hempel leg.

Em Myrtaceae.

21. Apiococeus gregarius Hempel.

Apiococcus gregarius Hempel, Rev., IV, p. 402, 1900. Hab. Estado de S. Paulo, Ypiranga.

Mus. Paul. 15.639, Ypiranga, S. Paulo, Hempel leg. Typo.

Em Myrtaceae. 
25. Apiococeus singularis Hempel.

Apiococcus singularis Hempel, Rev., IV, p. 403, 1900. Hab. Estado de S. Paulo. Ypiranga.

Mus. Paul. N. 19, Ypiranga, S. Paulo, Hempel leg. Tyрo.

Em Myrtaceae.

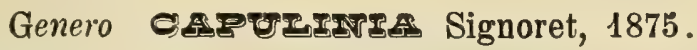

26. Capulinia crateraformans Hempel.

Capulinia crateraformis Hempel, Can. Ent., XXXII, p. 3, 1900.

Capulinia crateraformans Hempel, Rev., IV, p. 395, 1900. Hab. Estado de S. Paulo, Campinas, Itatiba, S. Paulo, Estado de Minas Geraes, S. João del Rei.

Mus. Paul. N. 369, S. João del Rei (Est. de Minas Geraes) Alvaro da Silveira leg.; N. 374, Estação Alto da Serra (Est. de S. Paulo) Hempel leg.

Em galhos de Eugenia jaboticaba.

27. Capulinia jaboticabae $v$. Ihering.

Capulinia jaboticabae v. Ihering, Rev. Agr., p. 188, 1898 ; Rev. III, 1898 p. 46-49.

Capulinia jaboticabae Hempel, Rev., III, p. 51, 1898, Capulinia jaboticabae Hempel. Rev., IV, p. 394, 1900.

Hab. Estado de S. Paulo. Ypiranga, S. Paulo, Campinas, Gapoeira Grande, Mogy-Guassú.

Mus. Paul. N. 214, Capoeira Grande (Est. de S. Paulo) Hempel leg. Co-typo.

Em Eugenia jaboticaba.

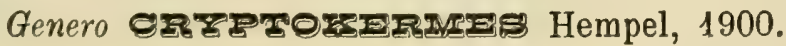

28. Cryptokormes brasiliensis Hempel. 1906.

Cryptokermes brasiliensis Hempel, Rev., IV, p. 398,

Hab. Estado de Minas Geraes. Poços de Caldas.

Mus. Paul. N. 286, Poços de Caldas (Est. de Minas Geraes), Typo; N. 380, 15.642, idem.

Em Schinus sp. 


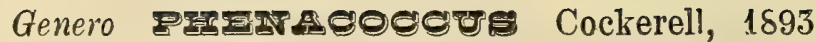

\section{Phenacoceus spiniferus Hempel.}

Phenacoccus spiniferus Hempel. Rev., IV, p. 389, 1900. Hab. Estado de S. Paulo, S. Paulo.

Mus. Paul. N. 336, S. Paulo (cidade) Hempel leg. Typo. Em uma arvore cultivada.

\section{Genero z d}

30. Lachnodiella cecropiae Hempel.

Lachnodiella cecropiae Hempel, Rev. VIII, p. 52, 1910. Hab. Estado de S. Paulo. Campinas, S. Paulo.

Mus. Paul. N. 11.599. Estação Raiz da Serra (Est. de S. Paulo) Luederwaldt leg.

No interior de Cecropia adenopus.

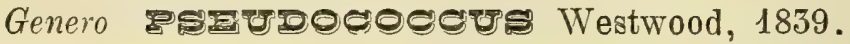

\section{Pseudocoreus bromeliae (Bouché)} 1833.

Lecanium bromeliae Bouché, Schäd. Gart. In., p. 49,

Hab. Estado de Piauhy, Itagybá; India, Zanzibar, Africa do Sul, America do Norte.

Em Abacaxi, amoreira, Canna e Hibiscus.

32. Pseudococcus citri (Risso).

Dorthesia citri Risso, Essai, Hist. Nat. des Oranges. 1813. Dactylonius citri Hempel, Rev., IV, p. 384, 1000.

Hab. Estado de S. Paulo, Campinas; Estado do Rio Grande do Sul, Colonia Novo Mundo; Jamaica, Europa, America do Norte, Ilhas Hawai, Mauricia.

Em Citrus, cafeeiro, fumo, algodão, Ipomoea, Solanum e outras plantas.

\section{Pseudococeus grandis (Hempel)}

Dactylopius grandis Hempel. Rev., IV, pag. 384, 1900. Hab. Estado de S. Paulo, Ypiranga.

Mus. Paul. N. 342, Ypiranga, (S. Paulo) Hempel leg. Typo; N. 16.635, idem.

Em Psidium sp. 

King).

34. Pseudococeus magnolicida ("von Ihering,"

Dactylopius magnolicida King, Rev., V, p. 616, 1902. Hab. Brazil.

35. Pseudococeus secretus (Hempel).

Dactylopius secretus Hempel, Rev., IV, p. 387, 1900. Hab. Estado de S. Paulo, Ypiranga. Typo.

Mus. Paul. N. 346, Ypiranga, S. Paulo, Hempel leg.

Em Solanaceae.

36. Pseudococeus setosis (Hempel).

Dactylopius setosus Hempel. Rev. IV, p. 386, 1900.

Hab. Estado de S. Paulo, S. Paulo, Estado do Rio de Janeiro, Praia de Botafogo. Typo.

Mus. Paul. N. 345, Ypiranga, S. Paulo, Hempel leg. Em Ficus sp. e Sapindus saponaria.

Genero

37. Erium armatum (Hempel).

Eriococcus armatus Hempel, Rev. IV, p. 383, 190().

Hab. Estado de S. Paulo, Ypiranga.

Mus. Paul. N. 110, Ypiranga, S. Paulo, Pinder leg. Typo. Em Baccharis sp.

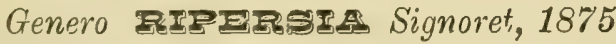

38. Tripersia taquarae n. sp. $\left({ }^{*}\right)$

Hab. Estado de S. Paulo, Alto da Serra. Em taquara-uçú.

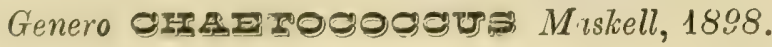

39. Chaetococeus bambusae (Masksll).

Sphaerococcus bambusae Maskell, N. Z. Trans., XXV, p. 237, 1892.

(*) Veja-se a descripção no fin dest, catalogo. 
Chaetococcus bambusae Gkll., Rev. III, p. 501, 1898.

Chaetococcus bambusae Hempel, Rev., IV, p. 397, 1900.

Hab. Estado de S. Paulo, Campinas, Ceylão, Ilhas Hawai, Mauricia.

Mus. Paul. N. 15.637, Campinas (Est. de S. Paulo), Dr.

F. Noack leg.

Em bambú cultivado.

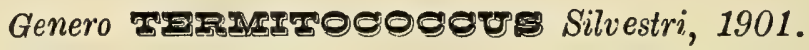

40. Termitococeus brevicornis Silvestri.

Termitococcus brevicornis Silvestri, Boll. Mus. Torino, XVI, p. 4, 1901.

Hab. Estado de Matto Grosso, Cuyabá.

Em ninhos de cupim.

\section{Sub-Familia TACHARDIINAE}

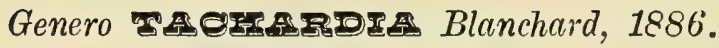

\section{Tachardia caerulea Henipel.}

Tachardia caerulea Hempel, Bol. Agri., V, p. 314, 1904. Hab. Estado do Rio de Janeiro, Mauá.

Mus. Paul. N. 16.291, Rio de Janeiro, Hempel leg.

Em uma arvore sylvestre.

42. Tachardia eydoniao Hempel.

Tachardia cydoniae Hempel, Rev., IV, p. 410, 1900. Hab. Estado de S. Paulo, S. Paulo.

Mus. Paul. N. 267, S. Paulo (cidade) Typo.

Em ramos de marmelleiro cultivado, Cydonia sp.

\section{Tachardia ingao Hempel.}

Tachardia ingae Hempel, Hev. IV, p. 415, 1900. Hab. Estado de s. Paulo, Mogy-Guassú.

Mus. Paul. N. 388, Mogy-Guassú (Est. de S. Paulo) Hempel leg. Typo; N. 15.67y, Cachoeira (Est. de S. Paulo) Hempel leg.

Em galhos de Inga sp. 


\section{Tachardia parva Hempel.}

Tachardia parva Hempel, Rev. IV, p. 413, 1900. Hab. Estado de S. Paulo, Ypiranga, Cachoeira. Mus. Paul. N. 15 646, Cachoeira (Est. de S. Paulo) Typo. Em Myrtaceae.

\section{Tachardia rosae Hempel.}

Tachardia rosae Hempel, Rev. IV, p. 414, 1900. Hab. Estado de S. Paulo, S. Paulo.

Mus, Paul. N. 332, S. Paulo (cidade) Hempel leg. Typo. Em ramos de roseira cultivada.

\section{Tachardia rubra Hernpel.}

Tachardia mbra Hempel, Rev. IV, p. 111, 1800.

Hab. Estado de S. Paulo, Cachoeira, Villa Americana. Mus. Paul. N. 15.648, Cachoeira (Est. de S. Paulo). Hempel leg. Typo; N. 282, Santa Barbara (Est. de S. Paulo); 15.647, Est. do Rio Grande do Sul, Dr. J. Dutra leg.

Em Croton sp.

\section{Sub-Familia COCCINAE}

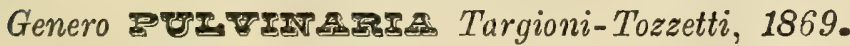

47. Pulvinaria depressa Hempel.

Pulvinaria depressa Hempel, Rev. IV, p. 490, 1900.

Hab. Estado de S. Paulo, Ypiranga.

Mus. Paul. N. 15.668, Ypiranga, S. Paulo, Hempel leg. Typo.

Em Miconia sp.

\section{Pulvinaria eugeniae Hempel.}

Pulvinaria eugeniae Hempel, Rev. IV, p. 488, 1900.

Hab. Estarlo de S. Paulo, Ypiranga, S. Paulo.

Mus. Paul. N. 290, S. Paulo (cidade) Typo; N. 257, Ypiranga, S. Paulo, Hempel leg. taceae.

Em Eugenia jaboticaba e outros arbustos da ordem Myr- 
49. Palvinaria ficus Hempel.

Pulvinaria ficus Hempel, Rev., IV, p. 486, 1900. Hab. Estado de S. Paulo.

Mus. Paul. Ns. 318 e 15.667, S. Paulo (cidade) Typo. Em Ficus sp., Psidium sp., Mangifera indica, e Ixora coccinea.

50. Pulvinaria grandis Hempel.

Pulvinaria grandis Hempel, Rev., p. 491, $19 \mathrm{CO}$.

Hab. Estado de S. Paulo, Ypiranga.

Mus. Paul. N. 270, Ypiranga, S. Paulo, Typo.

Em Myrcia sp., e outras plantas da ordem Myrtaceae.

\section{Pulvinamia ormata $n . s p .\left({ }^{*}\right)$}

Hab. Estado de S. Paulo. Campinas.

Mus. Paul. N. 16.29j, Campinas (Est. de S. Paulo), Hempel leg. Co-typo.

Em planta trepadeira Arrabidaea sp. fam. Bignoniaceae.

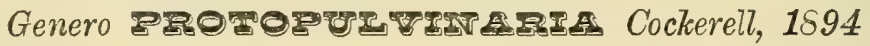

52. Frotopulvinaria convexa Hempel. 1900.

Protopulvinaria convexa Hempel, Rev., IV, p. 485,

Hab. Estado de S. Paulo. Republica Argentina.

Mus. Paul. N. 327, S. Paulo (cidade) Hempel leg. Typo. Em Smilax sp.

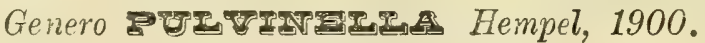

53. Pulinella puleholla Hempel.

Pulvinaria (Pulvinella) pulchella Hempel, Can. Ent., XXXl, p. 132, 1899.

Pulvinella pulchella Hempel, Rev., IV, p. 481, 1900.

Hab. Estado de S. Paulo, Ypiranga.

Mus. Paul. N. 265, Ypiranga, S. Paulo, Hempel leg. Tуро.

Em Baccharis dracunculifolia D. C.

(*) Vej\&-se \& discripção no fim deste catalogo. 


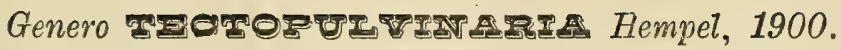

54. Tectopulvinaria albata Hempel,

Tectopulvinaria albata Hempel, Rev., IV, p. 483, 1900. Hab. Estado de S. Paulo. Ypiranga, Jundiahy, Campinas. Mus. Pau?. N. 15.C38, Jundiahy, Schrottky leg.; N. 15.669, Ypiranga; N. 15.670, S. Pailo.

Em Vernonia polyanthus Less. e Trichogonia salviaefolia.

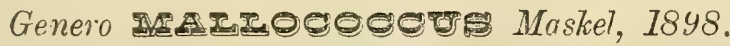

55. Mallococerus lamigering (Hemptl).

Lecaniurn lanigerum Hempel, Rev., IV p. 446, 1900. Guassú.

Hab. Estado do S. Paulo, Itapira, margens do rio Mogy-

Mus. Paul. N. 387, Rio Mogy-Guassú (Est. de S. Paulo) Hempel leg. Typo.

Em um arbusto sylvestre.

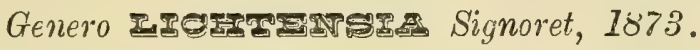

56. Lichtensia an entata Hempel.

Lichtensia argentata Hempel, Rev., IV p. 392, 1901). Hab. Estado de S. Paulo. Ypiranga.

Mus. Paul. N. 16.237, Ypiranga, S. Paulo, Hempel leg. Typo.

Em Ilicinae.

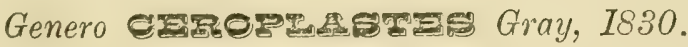

57. Ceroplastes albollimeatus Cockerell. 1894.

C'eroplastes albolineatus Ckll., Ent. News, V, p. 157,

Ceroplastes albolineatus Gkll., Rev., II, p. 70, 1897.

Ceroplastes albolineatus Hempel, Rev., IV, p. 474, 1900.

Hab. Estado de S. Paulo, Ypiranga, Jamaica, Mexico.

Mus. Paul. N. 12.914, Franca (Estado de S. Paulo); N. 15.676, S. Paulo; N. 15.677, Ypiranga, Ilempel leg.

Em Baccharis, Schinus, Fuchsia, Maytenus. 
58. Ceroplastes amazonicus Hempel.

Ceroplastes amazonicus Hempel, Rev., IV, p. 454, 1900, Hab. Estado de Amazonas, Manáos.

Mus. Paul. N. 443 e 15.68\%, Manáos (Estado do Amazonas) Bicego leg. Typo.

Provavelmente em planta sylvestre.

\section{Ceroplastes bicolor Hempel.}

Ceroplastes bicolor Hempel, Ann. Mag. N. H., (7), Vlll, p. 390,1901 .

Hab. Estado de S. Paulo, Campinas.

Em arvore do matto, não identificada.

60. Ceroplastes campinensis Hempel.

Ceroplastes campinensis Hempel, Ann. Mag. N. H., (7), VIII, p. 389, 1901.

Hab. Estado de S. Paulo, Campinas.

Em Psidium sp.

61. Ceroplastes cassiae (Chrvannes)

$1 \dot{11}, 1848$.

Coccus cassiae Chav., Ann. Soc. Ent. Fr., (2), VI, p.

Ceroplastes cassiae Ckll., Rev., II, p. 70, 1 , 997.

Ceroplastes cassiae Hempel, Rev., IV, p. 452, 1900.

Hab. Estado do Rio de Janeiro, Europa, Antigua.

Em Bursera gummifera, Cassia, sp.

62. Ceroplastes communis Hempel.

Ceroplastes communis Hempel, Rev., IV, p. 459, 1900. Hab. Estado de S. Paulo, Ypiranga. Tyро.

Mus. Paul. N. 281, Ypiranga, S. Paulo, Hempel leg.

Em Maytenus sp.

63. Coroplastes connâtuens Cockerell \& Tinsley.

Ceroplastes confluens Gkll. \& Tins., Journ. Ins. Jam.. II, p. 168, $18 y$ \%. 
Ceroplastes confluens Hempel, Rev., IV, p. 460, 1900.

Hab. Estado de S. Paulo, Cachoeira, Ypiranga, MogyGuassú, Jamaica.

Mus. Paul. N. 295, Jamaica; N. 412, S. Paulo, R. v. Ihering, leg.; N. 15.690, Mogy-Guassú (Estado de S. Paulo) Hempel leg.

Em Inga sp., Mimosa, sp., e outras plantas.

64. Ceroplastes cultus Hempel.

Ceroplastes cultus Hempel, Rev. IV, p. 470, 1900.

Hab. Estado de S. Paulo, Ypiranga. Typo.

Mus. Paul. N. 15.675, Ypiranga, S. Paulo, Hempel leg.

Em Erigeron canadensis L.

65. Ceñolastes cuneatus Hempel.

Ceroplastes cuneatus Hempel, Rev. VI, p. 471, 1900.

Hab. Estado de S. Paulo, Ypiranga.

Mus. Paul. N. 15.673, Ypiranga, S. Paulo, Hempel leg. Typo.

En Erigeron canadensis L.

E6. Ceroplastes fairmairii Targioni-Tozzetti.

Ceroplastes fairmairii Targ. Toz., Cat., p. 25, 1869.

Hab. Rio de Janeirc, Montevideo.

Em Cravo da India.

67. Ceroplastes floridensis Comstock.

Ceroplastes flomdensis Comstock, Rep. U. S. Dept. Ag., 1880 , p. 331, $18,81$.

Ceroplastes floridensis Hempel, Rev., IV, p. 462, 1900.

Hab. Estado de S. Paulo, S. Paulo, America do Norte, India, Japão, Australia, Ilhas Hawai, Ceylão.

IIus. Paul. N. 15.683 e 15.688, S. Paulo, Hempel leg.; N. 15.689, Panamá.

Em Ficus sp., Hedera sp., Citrus, Mangifera, Anona reticulata, Psidium, etc.

68. Ceroplastes formicarius Hempel.

Ceroplastes formicarius IIempel, Rev., IV., p. 472, 1900. 
Hab. Estado de S. Paulo, Ypiranga.

Mus. Paul. N. 15 684, Ypiranga, S. Paulo, Hempel leg. Typo.

Em Maytenus sp.

69. Ceroplastes formosus Hempe?.

Ceroplastes formosus Hempel, Rev., IV, p. 468, 1900. Hab. Estado de Minas Geraes, Poços de Caldas. Mus. Paul. N. 15.672, Poços de Caldas, (Estado de Minas Geraes) Hempel leg. Typo.

Em ramos de Eugenia sp.

70. Ceroplastes grandis Hempel.

Ceroplastes grandis Hempel, Rev., IV, p. 455, 1900. Hab. Estado de S. Paulo, Ypiranga, S. Paulo.

Mus. Paul. N. 15.678, Ypiranga, S. Paulo, Hempel Jeg. Typo.

Em Zanthoxylum, Ilex, Psidium, Baccharis, Platanus, Mechilia flava.

\section{Ceroplastes iheringi Cockerell.} 1895.

Ceroplastes iheringi Cockerel, The Ent, XXVIII, p. 100,

Ceroplastes iheringi Cockerell, Rev., II, p. 70, 1897.

Ceroplastes iheringi Hempel, Rev., IV, p. 452, 1900.

Hab. Estado de S. Paulo, Ypiranga, Campinas, Cachoeira, Itapira, Gapoeira Grande, Estado do Rio Grande do Sul. Mus. Paul. N. 274, 14.429 e 15.681, Ypiranga, S. Paulo, Co-typo ; 15.533, Alto da Serra (Est. de S. Paulo).

Em Baccharis platensis e Baccharis dracunculifoliae.

72. Ceroplastes janeirenses Gray.

Ceroplastes janeirenses Gray, Spic., Zool., p. 7, 1830. Ceroplastes janeirensis Hempel, Rev., IV, p. 451, 1900. Hab. Estado de S. Paulo, Ypiranga, Campinas, Europa. Mus. Paul. N. 5), Ypiranga, S. Paulo, Dr. H. v. Thering, leg.; N. 12.887, idem, Luederwaldt leg.

Em Psidium sp. e outras plantas da ordem Myrtaceae. 
73. Ceroplastes Incidus Hempel.

Ceroplastes lucidus Hempel, Rev., IV, p. 465, 190 n. Hab. Estado de S. Paulo, Ypiranga. Typo.

Mus. Paul. N. 251, Ypiranga, S. Paulo, Hempel leg.

Em Baccharis dracunculifolia.

\section{Ceroplastes novaesi Hempel.}

Ceroplastes novaesi Hempel, Rev., IV, p. 457, 1900.

Hab. Estado de S. Paulo, Ypiranga, Campinas, Capoeira Grande, Cachoeira.

Mus. Paul. N. 248, Cachoeira (Est. de S. Paulo) Hempel leg. Typo ; N. 15.68\%, Ypiranga, S. Paulo.

Em Abutzlon, Baccharis dracunculitolia, Büccharis sp. - Vernonia riedelii.

\section{Ceroplastes psidii Chavannes.}

Ceroplastes psidii Clavannes, Ann. Soc. Ent. Fr., (2) VI, p. $139,1848$.

Hab. Rio de Janeiro, Est. de S. Paulo, Europa.

Mus. Paul. N. 15.680, Campinas (Est. de S. Paulo) Hempel leg.

Em Psidium sp.

76. Ceroplastes purpureus Hempel.

Ceroplastes purp'rreus Hempel, Rev., IV, p. 466, 1900. Hab. Estade de S. Paulo, Ypiranga.

En Moconia sp.

Mus. Paul. N. 292. Ypiranga, S. Paulo, Hempel leg. Typo.

77. Ceroplastes $\Theta \mathbf{x c a e c a r i a e} n . s p$.

Hab. Estado de S. Paulo, Ypiranga.

Mus. Paul. N. 16.611, Ypiranga, H. Luederwaldt leg. Typo.

Sobre Euphorbiacea, Excaecaria biglandulosa, M. Arg.

(*) $V \in j a-\varepsilon \theta$ a deseripçăo no fim deste całalogo. 
78. Ceroplastes rarus Hempel.

Ceroplastes rarus Hempel, Rev., IV, p. 469, 1900. $H a b$. Estado de S. Paulo, Ypiranga.

Mus. Paul. N. 25. Ypiranga, S. Paulo, Hempel leg. Typo. Em uma planta sylvestre.

\section{Ceroplastes rotundus Hempel.}

Ceroplastes rotundus Hempel, Rev., IV, p. 473, 1900. Hab. Estado de S. Paulo. Ypiranga.

Mus. Paul. N. 15674. Ypiranga, S. Paulo, Typo.

Em Maytenus sp.

80. Coroplastes simplex. Hempel.

Ceroplastes simplex Hempel, Rev., p. 475, 1900. Hab. Estado de S. Paulo. Ypiranga

Mus. Paul. N. 402. Ypiranga, S. Paulo, Dr. H. v. Ihering leg. Typo.

Em Myrtaceae.

81. Ceroplasios speciosus Hempel.

Ceroplastes speciosus Hempel. Rev., IV, p. 464, 1900. Hab. Estado de S. Paulo, Ypiranga.

Mus. Paul. N. 1568E. Ypiranga, S. Paulo, Hempel leg. Typo.

Em Myrtaceae.

82. Ceroplastes variegatus Hempel.

Ceroplastes variegutus Hempel, Rev. IV, p. 462, 1900. Hab. Estado de S. Paulo, Ypiranga. Mus. Paul. N. 207 e 403, Y piranga, S. Paulo, Hempel leg. Typo.

Em Miconia sp.

Genero ris

\section{Vinsonia stellifera (Westwood)}

Vinsonia stellifera, Hempel, Rev.. IV, p. 477, 1900. 
Hab. Rio de Janeiro e Pará, Barbados, America do Norte, etc.

Mus. Paul. N. 10்296. Rio de Janeiro, Hempel leg.

Em Lucuma caimito, mangosten, avenca, Orchideae, mangifera, Cocus nucifera, nós moscado.

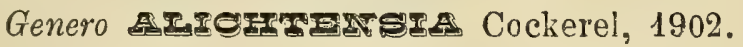

84. Alichtensia attenuata (Hempel).

Lichtensia (?) attenuata Hempel, Rev., IV, p. 194, 1901, Hab. Estado de S. Paulo. Ypiranga.

Mus. Paul. N. 16.239. S. Paulo, Typo.

Em Baccharis genistelloides var. trimera Baker.

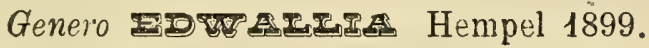

\section{Edwallia rugosa Hempel.}

Edwallia rugosa Hempel, Can. Ent., XXXI p. 131, 1899. Edwallia rugosa Hempel, Rev., IV, p. 478, 1900. Hab. Estado de S. Paulo. S. Paulo. Mus. Paul. N. 288. S. Paulo (cidade) Hempel leg. Typo. Em Eugenia jaboticaba.

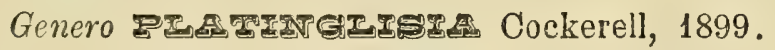

86. Platimglisia noacki Cockerell.

Platinglisia noacki Ckll., The Ent., XXXII, p. 12, 1899. Platinglisia noacki Hempel, Rev., IV, p. 477, 1900.

Hab. Estado de S. Paulo, Campinas, S. Paulo, Alto da Serra, S. José dos Campos.

Mus. Paul. N. 280, Estação Alto da Serra, Hempel leg. Em Laurus, Euphorbeacea, Thymeleacea e Myrtacea.

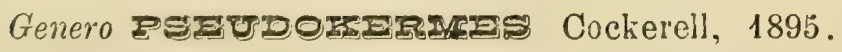

87. Psoudokermes nitons (Cockerell).

Lecanium (Pseudokermes) nitens Ckll., Can. Ent., XXVII, p. 203, 1895.

Pseudokermes nitens IIempel, Rev., IV, p. 448, 1900.

Hab. Estado de S. Paulo, Campinas, S. Paulo.

Mus. Paul. N. 285, Ypiranga, S. Paulo.

Em Myrtus (Blephorocalya) tweedii, Psidium sp. 


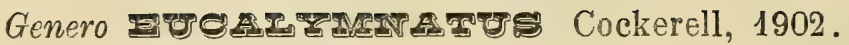

88. Eucalymmatus brunfelsiae (Hempel)

Lecanium brun (felsiae) Hempel, Rev., IV, p. 418, 1800. Hab. Estado de S. Paulo, Pilar, Alto da Serra.

Mus. Paul. N. 264. Estação Pilar (Est. S. Paulo) Typo. Em Brunfelsia sp.

89. Eucalymnatus gracilis (Hempel).

Lecanium gracile Hempel, Rev., IV, p. 419, 1900. Hab. Estado de S. Paulo, Villa Americana.

Mus. Paul. N. 15655. Sta. Barbara (Est. S. Paulo) Typo. Em Sapindaceae.

90. Eucalymatus performtus (Newstead).

Lecanium perforatum Newst, Ent. Mon. Mag., XXX, p. 233, 1894.

Hab. Rio de Janeiro, Inglaterra, Ilhas Hawai, Australia, America do Norte.

Mus. Paul. N. 16.897. Rio de Janeiro, Hempel leg.

Em Areca oleracea, Caryota cumingii, Eugenia jambos, Eevea belmoreana, Trachycarpus excelsus, etc.

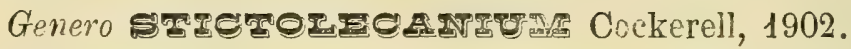

91. Stictolecanium ormatum (Hempel).

Lecanium ornatum Hempel, Rev., IV. p. 421, 1900. Hab. Estado de S. Paulo, S. Paulo.

Em Eugenia jaboticaba.

Genero cecers Linnaeus, 1758.

92. Coccus hesperidum Linn. 1758.

Coccus hesperidum L., Syst. Nat., Ed. X, i, p. 455 ,

Lecanium hesperidum Hempel, Rev., IV, p. 436, 1900.

Hab. Estado de S. Paulo, Ypiranga, Capoeira Grande, Europa, Australia, Nova Zelandia, Africa do Sul, ilhas Hawai, Japão, Algeria, Chile, America do Norte. 
Mus. Paul. N. 15.660. Campinas (Est. S. Paülo) Dr. F. Noack leg.

Em Citrus, Clematis flammula, Convolvulus tricolor, Mimosa, Nerium, Abutilon, Cycas revoluta, Morus, Phlox, etc.

\section{Cocens viridis (Green).} 1889.

Lecanium viride Green, Ent. Mon. Mag., XXV, p. 248,

Lecanium viride Hempel, Rev., IV, p. 434, 1900.

Hab. Estado de S. Paulo, Campinas, Ceylão, Mauricia.

Mus. Paul. N. 15.662, Campinas (Est. de S. Paulo) Dr. F. Noack leg.

Em Cinchona, Citrus, Gardenia, Psidium, café e chá da India.

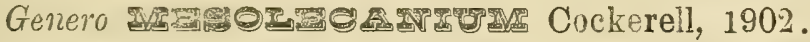

94. Mesolecanium hacelnaridis (Cockerell). 1895

Lecanium baccharidis Gkll., Am. Nat., XXIX, p. 174,

Lecanium baccharidis Iher., Rev. II, p. 4')8, 1897.

Lecanium baccharidis Hempel, Rev., IV, p. 435, 1900. Hab. Estado de S. Paulo, Ypiranga.

Mus. Paul. N. 271. Ypiranga, S. Paulo.

En Baccharis dracunculifolıa D. G.

95. Mesolecaninm (?) campomanesiae (Hempel)

Lecanium campomanesiae Rev.. IV, p. 447, 1900.

Hab. Estado de S. Paulo, Ypiranga.

Mus. Paul. N. 258, S. Paulo, Typo.

Em Campomanesia sp.

96. Mesolecanium inflatum Hempel.

Mesolecanium inflatum Hempel, Bol. Agr. V, p. 316, 1904. Hab. Rio de Janeiro.

Mus. Paul. N. 26298, Rio de Janeiro, Hempel leg.

Em Myrtacece. 
97. Mesolecanium lucidum $n$. $s p$.

Hab. Est. Rio Grande do Sul, Porto Alegre.

Mus. Paul. N. 16.600, Porto Alegre, Typo, Paldaof leg. Em Solanacea.

98. Mesolecanium (2) jaboticabae (Hempel). Lecanium jaboticabce Hempel, Rev.. IV, p. 443, 1900. Hab. Estado de S. Paulo, Ypiranga.

Em Eugenia jaboticaba.

99. Mesolecanium mayteni (Hempel)

Lecanium mayteni Hempel, Rev,, IV, p. 438, 1900. Hab. Estado de S. Paulo. Ypiranga, Jundiahy. Mus. Paul. N. 15664, Ypiranga, S. Paulo, Typo.

Em Maytenus sp.

100. Mesolecanium (?) obscurum (Hempel)

Lecanium obscurum Hempel, Rev., IV, p. 441, 1900. Hab. Estado de S. Paulo, Ypiranga.

Mus, Paul. N. 283 (Typo) 14331, Ypiranga ; N. 15653,

S. Paulo, Dr. H. von Ihering leg.

Em Maytenus sp.

101. Mesolecanium pseudlosemen (Cockerell) 1895 ,

Lecanium pseudosemen Ckll., Can. Ent., XXVII, p. 202,

Lecanium pseudosemen Gkll., Rev., II, p. 70, 1897.

Lecanium pseudosemen Hempel, Rev., IV, p. 444, 1900.

Hab. Estado de S. Paulo, Ypiranga, Jundiahy, Estado de Minas Geraes, Poços de Caldas.

Mus. Paul. N. 15654, S. Paulo (cidade); N. 15656, Poços de Caldas (Est. Minas Geraes).

Em Solanum paniculatum L.

102. Mesolecamium rhizophorge (Cockerell)

Lecanium rhizophorce Gkll., Rev., III, p. 501, 1898. Lecanium rhizophorce Hempel, Rev. IV, p. 437, 1900. Hab. Estado de S. Paulo, Cubatão, Em Rhizophora mangle.

(*) Veja se a descripçào no fia déste cat.l.go. 
Genero serer 1901.

\section{Neolecanium perconvexum (Cockerell)} 1898.

Lecanium perconvexum Ckll., The Ent. XXXI, p. 132,

Lecanium perconvexum Ckll., Rev., III, p. 41, 1898.

Lecanium perconvexum Hempel, Rev., IV, p. 422, 1900.

Hab. Estado de S. Paulo, Campinas, S. Paulo; Est. Minas Geraes, Poços de Galdas.

Mus. Paul. N. 205 Campinas (Est. S. Paulo) Dr. F. Noack leg. ; N. 15661, Poços de Caldas (Est. Minas Geraes).

Em Nectandra.

\section{Neolecanium silveirai (Hempel)}

Lecanium silveirai Hempel, Can. Ent. XXXII, p. 5, 1900, Lecanum silveirai Hempel, Rev., IV, p. 424, 1900.

$H a b$. Estado de Minas Geraes, Sete Lagoas, Diamantina. Mus. Paul. N. 35y, S. João del Rei (Est. Minas Geraes), Alvaro da Silveira leg. Typo.

Em raizes de videira cultivada.

\section{0מ̆. Neolecanium urichi (Cockerell)}

Lecanium urichi Gkll., Ent. News, V, p, 203, 1894.

Lecanium urichi Hempel, Rev., IV, p. 423, 1900.

Hab. Estado de S. Paulo, Campinas, Rio Grande do Sul Trindade e as Antilhas.

Em Smilax campestris Gr. e na casa de uma formiga, Crematogaster brevispinosa Mayr. var.

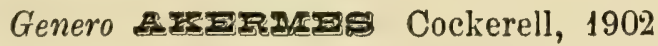

\section{6. kermen monilis (Cockerell)}

Lecanium monile Ckll, Can. Ent., XXVII, p. 203, 1 1395. Lecanium monile Ckll. Rev, II, p. 70, 1897.

Lecanium monile Hempel, Rev., IV, p. 446, 1900.

Hab. Estado de S. Paulo, (S. Paulc).

Mus. Paul. N. 15658, S. Paulo (cidade), Dr. H. v. Ihering leg.

Em uma planta não identificara. 


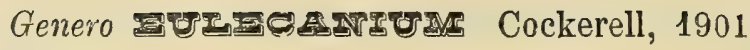

\section{Eulleranium eugeniae (Hempel).}

Lecanium eugenice Hempel, Rev., IV, p. 439, 1900.

Hab. Estado de S. Paulo, Ypiranga.

Mus. Paul. N. 263-15649, Ypiranga, S. Paulo, Typo. Em Eugenia sp.

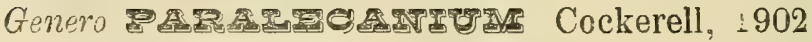

\section{Pardecanium marianum Cockerell}

Paralecanium marianum Ckll., Ann. Mag. N. H., (7) IX, p. 455, 1902.

Hab. Mauá, Rio de Janeiro.

Mus. Paul. N. 16292, Ris de Janeiro, Hempel leg.

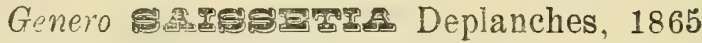

103. Taissetia depross (Targioni-Tuzzetti).

Lecanium depressum Targ., Studii sul Cocc. p. 29, 1867.

Lecanium nigrum var. depressum Hempel, Rev., IV, p. 425, 1900.

Hab. Estado de S. Paulo, Campinas, Pará, Europa, Australia, Ilhas Hawai, etc.

Em Hibiscus sabdariffa L., Ficus, Hakea, Psidium, Bambusa.

\section{Saissetiv discoides (Hempel)}

Lecanium discoides Hempel, Rev. IV, p. 433, 1900. Hab. Estado de S. Paulo, Ypiranga.

Mus. Paul. N. 15659, Ypiranga, S. Paulo, Typo.

Ein Psidum sp., e outras plantas da ordem Myrtaceae.

111. Saissetia dura (Hempel).

Lecanium durum Hempel, Rev., IV, p. 427, 1900. Hab. Estado de S. Paulo, Ypiranga. Mus. Paul. N. 15650 Ypiranga.

Em Baccharis dracunculifolia D. C. 
112. Saissotia ghamulasa (Himpl).

Lecanium glanulosum Hempel, Rev., IV, p. 428, 1900. Hab. Estado de S. Paulo, Ypiranga.

Mus. Paul. N. 15651 Ypiranga, S. Paulo, Typo.

Em Myrtacece.

11j. Saissetia hemisphaerica (Turgioni-Tozzetti). 26, 1867.

Lecanium hemiphaericum Targ., Studii del Cocc. p.

Lecanium coffece Hempel, Rev., IV, p. 426, 1900.

Hab. Estado de S. Paulo, Campinas, Poço Grande, Bahia, Pará, Europa, Australia, Mauricia, America do Norte, Mexico, etc. pel leg.

Mus. Paul. N. 161 Poço Grande, (Est. S. Paulo) Hem-

Cycas, Psidium, café, Citrus Nerium, Camellia. etc.

114. Saissetia infrequens (Hempei).

Lecanium infrequens Hempel, Rev. IV, p. 431, 1900. Hab. Estado de S. Paulo, Ypiranga.

Mus. Paul. N. 371, Ypiranga, S. Paulo, Hempel leg. Typo. Em Zanthoxylum sp.

115. Saissetia lucida $n \cdot s p \cdot\left(^{*}\right)$

Hab. Estado de S. Paulo, Alto da Serra.

Mus. Paul. N. 155:34 Parque Cajurú, Alio da Serra (Est. de S. Paulo) Schwebel leg. Typo.

Em uma arvore sylvestre.

116. Saissetia olese (Bernard).

Chermes olece Bern., Mem., d'Hist. Nat. Ac., Mar., p. 108, 1782 .

Lecanium olece Hempel, Rev., IV, p. 425, 1900.

Hab. Estado de S. Paulo, Ypiranga, Campinas, Europa, America do Norte, China, Japão, Australia, Africa, etc.

Mus. faul. N. 21 e 15661 , Ypiranga, S. Paulo.

Em Nerium, Citrus, I'sidium, Eucalyptus, Magnolia, pereira, macieira, videira, oliveira, etc.

(*) $\nabla \cdot j$ «-80 a d scripęão no fim deste ca'alogo. 
117. Saissetia reticulata (Cockerell). $18 \mathrm{c5}$.

Lecanium reticulatum Gkll., Am. Nat., XXIX, p. 174,

Lecanium reticulatum Ckll., Rev, II, p. 408, 1897.

Lecanium reticulatum Hempel. Rev., IV, p. 426, 1900. Hab. Estado de S. Paulo. Ypiranga.

Mus. Paul. N. 297 e 15666, Ypiranga, S. Paulo,

Em Myrtaceae.

118. Gaissetia zanthoxylum (Hempel).

Lecanium zanthoxylum Hempel. Rev., IV, p. 43), 1900. Hab. Estado de S. Paulo, Ypiranga.

Mus. Paul. N. 15657 Ypiranga, S. Paulo, Typo.

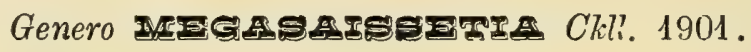

119. Megasaissetia brasiliensis $n$. sp. (*)

Hab. Est. S. Paulo, Ato da Serra.

Mus. Paul. Estação Biologica, Alto da Serra, N. 16604, Typo.

120. "Lecanium» erythrinae $v$. Ihering.

Lecanium erythrinae v. Ihering Rev. II. pag. 407, 1897. Lecanium erythrinae Hempel. Kev., IV. p. 438, 1900. Hab. Estado do Rio Grande do Sul.

Em Erythrina cristagalli L.

121. "Lecanium» insolens King.

Lecanium insolens. King, Rev. Chil. Hist. Nat. Vl, p. 255, 1902.

Hab. Brazil.

Em Philodendron.

\section{SUB-FAMILIA DIASPINAE}

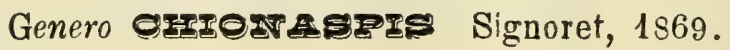

122. Chionaspis eitri Comstock.

Chionaspis citri Comstock. 2 R. Dep. Ent. Corn. Univ., p. $100,1883$.

Hab. Rio de Janeiro, Mexico, Australia, Japão, Samoa, Nova Zelandia. America do Norte.

Em Citrus, Euonymus latifolius, Osmanthus, palmeiras, etc.

(*) Veja-se a deccripção no fim desto estalrgo. 


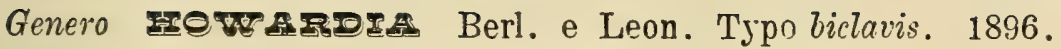

\section{Howardia bielavis Comstock.}

Chionaspis (?) biclavis Comstock, 2 nd. Report. Dept. Ent. Cornell University, p. 98, 1883.

Hab. America do Norte, Inglaterra, Tahity Ceylão, Japão, Ilhas Hawai, Mauricıa. Estado de S. Paulo, Campinas.

Em Ficus sp. Citrus, café, cha, tamarindo, Anona muricata, Hibiscus aculeatıs etc.

Genero DIA폴요 Costa, 1828.

124. Diaspis australis Hempel.

Diaspis australis Hempel, Rev,. IV, p. 521, 1900.

Hab. Estado de S. Paulo, Ypiranga. Typo.

Mus. Paul. N. 15.720, Ypiranga, S. Paulo, Hempel leg.

Em Myrtaceae.

\section{Diaspis boisduvalii Signuret.} 432, 186!).

Diaspis boisduvalii Sign., Arn. Soc. Ent. Fr. (4), IX, p.

Aulacaspis boisduvalii Hempel, Rev., IV, pag. 518, 1900.

Hab. Estado de S. Paulo, Capoeira Grande, Alto da Serra; Estado de Minas Geraes, Poços de Caldas. Europa, America do Norte, Australia, Mexico etc.

Mus. Paul. N. $26=16.241$, Ypiranga, S. Paulo, Hempel leg.; N. 329 Capoeira Grande (Est. de S. Paulo); N. 273 Poços de Caldas (Est. de Minas Geraes); N. 256, Estação Alto da Serra (Est. S. Paulo) Edwall leg.

Em Pleiochiton ebracteatum, Acacia, Cattleya, palmciras, etc.

126. Diaspis boisduvalii maculata (Cockerell).

Aulacaspis boisduvalii var. maculata Ckll., Rev., III, p. 502, 1898.

Aulacaspis boisduvalii var. maculata Hempel, Rev., IV, p. 518, 1900 .

Hab. Estado de S. Paulo. Campinas, Antigua.

Em Lamaceae, ananas. 


\section{Diaspis bromeliae (Kern.)} 52, 1778.

Coccus bromeliae Kern., Naturg. G. bromeliae, pp. 20,

Hab. Estado de S. Paulo, Europa, America do Norte, Mexico.

Mus. Paul. N. 12.886, Ypiranga, S. Paulo, Luederwaldt leg. Em Bromelia pinguin, Hibiscus, Canna, Billbergia zebrina, Olea fragrans, ananás, éra.

128. Diaspis cordiae Rübsamen.

Diaspis cordiae Rübs., Marcellia, IV, 5, p. 122, 1905. Hab. Rio de Janeiro.

Em Cordia curassavica.

\section{Diaspis echinocacti cacti Comstock.}

Diaspis cacti Comstock, 2nd. Rep. Dep. Ent. Corn. Univ., p. 91, 1883.

Diaspis cacti Hempel. Rev. IV, p. 520, 1900.

Hab. Rio de Janeiro. India, Mauricia, America do Norte.

Mus. Paul. N. 368, Rio de Janeiro, E. Ule leg.

Em Cereus macroconus, Cereus giganteus. Echinocactus.

Genero

150. Aulacagpis pontagona (Iargioni-Tuzzetti). $11,1885$.

Diaspis pentagona Targ. Revista di Bacchicoltura, N.

Diaspis pentagona Hempel. Rev. IV, p. 519. 1900.

Hab. Estado de S. Paulo, Campinas, Estado de Minas Geraes, S. João d'el Rei. Rio de Janeiro, America do Norte, Europa, Ceylão, Australia, Republica Aroentina.

Mus. Paul. N. 15.718, S. João del Rei (Est. Minas Ger.) Alv. da Silveira leg.; N. 15.719 , Est. Rio de Janeiro.

Em ameixeira, pecegueiro, videira, nogueira, Hibiscus ete.

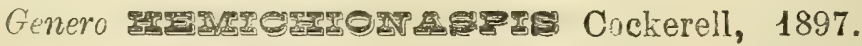

131. THemienionaspis aspidistrae (Signoret).

Chionaspis aspidistrae Sign., Ann. Soc. Ent. Fr., (4), IX, p. 443, 1869. 

1900.

Hemichionaspis aspidistrae Hempel, Rev. IV, p. 516,

Hab. Estado de S. Paulo, Ypiranga, S. Paulo. Europa, America do Norte, India, Formosa, Japão, Ceylão, Australia, Trindade.

Mus. Paul. N. 15.713, Ypiranga, S. Paulo.

Em Aspidistra lurida, Cocos plumosa, Cyanotus, Areca catechu, Acacia melanoxylon, Citrus, etc.

\section{Homichionaspis mimor (Maskell).} 1884.

Chionaspis minor Maskell, N. Z. Trans., XVII, p. 33,

Hemichionaspis minor Hempel, Rev. IV, p. 417, 1900.

Hab. Estado de S. Paulo. Garmpinas, Cachoeira. Rio de Janeiro. Nova Zelandia. Japão, Ceylão, Grenada, Antigua, Jamaica, Panama, America do Norte.

Mus. Paul. N. 14.449, Campinas (Est. S. Paulo); N. 15.715, Rio de Janeiro, E. Ule leg.

Em Melia azederack, Parsonia, Rhipogonum scandens, Hibiscus, Capsicum, Cycas revoluta, Cocos nucifera, Pelargonium, Albizzia, Agava, figueira, laranjeira, algodoeiro, Nerium.

\section{Henuichionaspis orlandi Leonardi.}

Hemichionaspis orlandi Leonardi, Redia, III, p. 5, 1906. Hab. Brazil.

Em uma planta não classificada.

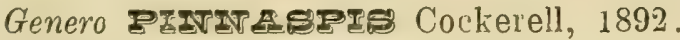

\section{Pinnaspis buxi (Bouché).} 1851.

Aspidiotus buxi Bouché, Stett. Ent. Zeit., XII, p. 111,

Hab. Rio de Janeiro, Europa, America do Norte, Jamaica, Trindade, Panama, Grenada.

Mus. Paul. N. 16.299, Rio de Janeiro, Hempel leg.

Em Buxus sempervirens, Dictyosperma album, Areca lutescens, Cocos nucifera, Pandanus conoidens, Anthurium cristallinum, Thrinas exclesa, Dracaena. 


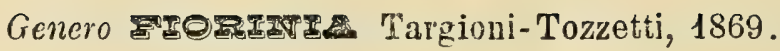

155. Fiorinia fiorimiae (Targioni-Tozzetti).

Diaspis froriniae Targ., Studi sul. Cocc., p. 14, 1867.

Fiorinia foriniae Hempel, Rev. IV, p. 509, 1900.

Hab. Estado de S. Paulo. Campinas. Europa, Ceylão, Australia, Ilhas Hawai, Mauricia, China, Jamaica, Barbados, Mexico, America do Norte.

Mus. Paul. N. 15.717, Campinas (Est. S. Paulo) Dr. F. Noack leg.

Em Hedera helix, Cocos nucifera, Camellia, Areca aurea, Cycas revoluta, Anthurium acaule, Phytelephas mxcrocarpa, Livistona, Kentia balmoreana, Leptospermum, Ficus, Podocarpus, Cupressus, chá, etc.

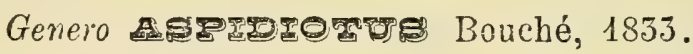

\section{Aspidiotus eyanophylli Signoret.}

Aspidiotus cyanophÿlli Sign., Ann. Soc. Ent. Fr.. (4), IX, p. 119, 1869.

Aspidiolus cyanophylli Hempel, Rev., IV, p. 498, 1900. Hab. Estado de S. Paulo. S. Paulo, Eurcpa, Ceylão, Mauricia, Mexico, America do Norte.

Mus. Paul. N. 373 S. Paulo, Dr. H. v. Ihering leg.

Em Laurus, Cyanophyllum, Ficus, Cycas revoluta, Ipomaea, Fritchardia filifera, palmeiras, orchideas, chá da India, Cinchona.

\section{Aspidiotus eydoniae Cumstock.}

Aspidiotus cydoniae Comstock, Rep. U. S. Dep. Ag., 1880 , p. 295, 188 !.

Hab. Estado de S. Paulo, Campinas, America do Norte, Mexico, Indias Occidentaes, Ceylão, samoa.

Mus. Paul. N. 560, S. João del Rei (Est. Minas Ger.) Alv. da Silveira leg.

Em Marmelleiro, figueira, palmeira, chá da India, Cactus, laranjeira, Jasminum, Lantana. 
138. spidiotus destructor Signoret.

Aspidiotus destructor Sign.. Ann. Soc. Ent. Fr., (4), IX, p. 120, 1869.

Hab. Rio de Janeiro, China, Formosa, India, Mexico, Bourbon, Mauricia, Demerara, Indias Occidentaes.

Mus. Paul. N. 16.301, Rio de Janeiro, Hempel leg.

Em Anona squamosa, Celtis occidentalis, Terminalia, palmeiras, mangueiras, bananeiras, noz moscardo.

\section{Aspidiotus Iataniae Signoret.} p. $124,1869$.

Aspidiotus lataniae Sign., Ann. Soc. Ent. Fr., (4), IX, 502, 1900.

Hab. Estado de Minas Geraes, Uberaba, S. João d'el Rei, Europa, Mauricia, Ilhas Galapagos.

Mus. Paul. N. 293 Est. Rio Gr. do Sul, Dr. J. Dutra leg; N. 15.ô99, Ypiranga S. Paulo, Dr. H. v. Ihering leg.; N. 15.700, S. Joãu del Rei (Est. Minas Geraes).

Em Videira, Latania, Areca lutescens, Scalesia, Cocos nucifera.

140. Aspidiotus moreirai Hempel.

Aspidiotus moreirai Hempel, Bol. Agri, V. p. 320, 1904. Hab. Rio de Janeiro.

Em Drimys sp.

\section{Mspidiotus orientalis Newsted.}

Aspidiotus orientalis Newstead, Ind. Mus. N., III, No. 5, p. 26, 1894.

Hab. Rio de Janeiro, Rio Grande do Sul, S. Carharina.

Em Myrtaceae, Podocarpus lamberti, Weinmannia sp., Myrrhinium rubriflorum, Orchideae.

\section{Aspidiotus pisai Hempel.}

Aspidiotus pisai Hempel. Bol. Agr., V, p. 320, 1904. Hab. Rio de Janeiro.

Mus. Paul. N. 16.300, Itatiaya (Est. Rio de Janeiro) Hempel leg.

Em Drimys sp 
143. Apidiotus rapax Comstock.

Aspidiotus camelliae Sign., Ann. Soc. Ent. Fr., (4) IX, p. 117, 1869, (nome preoccupado).

Aspidiotus rapax Comst., Rep. U. S. Dept. Ag., 1880, p. $307,1891$. 501,1900 ,

Aspidiotus (Hemiberlesia) camelliae Hempel, Rev., IV, p.

Hab. Estado de S. Paulo, Ypiranga, Rio de Janeiro. America do Norte, Indias Occidentaes, Europa, Ilhas Hawai, Nova Zelandia, Australia, Africa do Sul.

Mus. Paul. N, 32\%, 343, 15.721, Ypiranga, S. Paulo; N. 15.694, Uberaba (Est. Minas Ger.); N. 15.702 Rio de Janeiro.

Em Baccharis dracunculifolia, Erigeron canadensis, Trichogonia salviaefolia, Camellia, Acacia, Fuchsia, Eucalyptus, Myoporum, Rhamus crocea, Cercis, Coprosma, nogueira, oliveira, figueira, laranjeira, macieira, pereira, marmelleiro, chá da India.

Genero

\section{Morganella maskelli (Cockerell)}

Aspidiotus longispina Mask. (non Morg.), N. Z. Trans., XXVII, p. 38, 1894.

Aspidiotus (Morganella) maskelli Ckll., Bull. 6, T. S. Dept. Ag., p. 22, 1897.

Aspidiotus (Morganella) maskelli Hempel, Rev., IV p. 498, 1900 .

Hab. Estado de S. Paulo, Campinas; Mauricia, Ilhas Hawai.

Mus. Faul. N. 16302, Campinas (Est. de S. Paulo) Hempel leg.

Em Michelia flava, Camellia

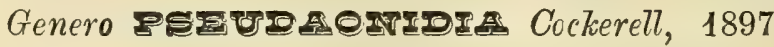

\section{Pseudaonidia trilobitiformis (Green.)}

Aspidiotus trilobitiformis Green, Ind. Mus. Notes, IV, p. 4. 1896.

Aspidiotus (Pseudaonidia) trilobitiformis Hempel, Rev., IV, p. 499, 1 c00. 
Hab. Estado de S. Paulo, Campinas, Rio de Janeiro, Bahia, Japão, Ceylão, Mauricia, Indias Orientaes.

Mus. Paul. N. 15690 Bahia; N. 16701 e 15703 Rio de Janeiro, Hempel leg.

Em Dalbergia championii, Ficus scandens, Citrus, Myrtacece, kaki, Anona, cajú.

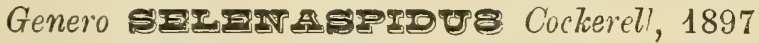

\section{Selenaspidus articulatus (Morgan)} $352,1889$.

Aspidiotus articulatus Morgan, Ent. Mon. Mag., XXV, p.

Aspidiotus (Selenaspidus) articulatus Hempel, Rev., IV, p. $499,1900$.

Hab. Pará, Mexíco, Panamá, Gosta Rica, Demerara, Indias Occidentaes, Africa, Inglaterra.

Em Cordiline terminalis, Pandanus, Gardenia, Ficus, Dictyosperma album, Citrus, cafeeiro.

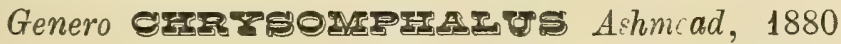

\section{Chrysomphalus aonidum (Linn.)}

Coccus aonidum Linn., Srst. Nat.. Ed. X I. p. 455, 1758. Aspidiolus (Chrysomphalus) aonidum Hempel, Rev. .V,
p. 502, 1900.

Hab. Estado de S. Paulo, Ypiranga, Campinas. Estado do Rio de Janeiro, Barra do Pirahy, Barbados, Jamaica, Japão. Australia, Mauricia, Africa, India, Ceylão, Europa, America do Norte.

Mus. Paul. N. 105 e 15 fr97, S. Paulo, (cidade); N. 15692, Campinas, (Est. S. Paulo).

Em Citrus, Hedera, Cocos nucifera, bananeira, Laurus, palmeiras, roseiras, Ilex latifolia, Ilex lurida, Camellia, Dictyosperma album, Rhododendron arboreum, Psidium, Ficus, Nerium, Begonia magnifica, etc.

\section{Chrysomphalus dictyospermi (Morgan)}

Aspidiotus dictyospermi Morgan, Ent. Mon. Mag. XXV, p. 352, 1889.

Aspidiotus (Chrysomphalus) dictyospermi Hempel. Rev., IV, p. 505, 1900. 
Hab. Estado de S. Paulo, Ypiranga, Rio de Janeiro, Mexico, America do Norte, Indias Occidentaes, Demerara, Ghina.

Mus. Paul. N. 413, Ypiranga, S. Paulo; N. 15693, Panamá.

Em Dictyospermum album, Erythrina indica, Cycas, Latania, Drymophloeus robustus, palmeiras, roseiras, mangueiras.

149. Chrysomphalus dictyospermi arecae (News'ead)

Aspidiotus dictyospermi areco Newst. Ent. Mon. Mag., XXIX, p. 185, 189:3. dentaes.

Hab. Brazil, Demerara, India, Inglaterra, Indias Occi-

Em Areca triandra, Cypripedium, Dendrobium, Anthurium, Aloe seyberi, chá da India.

150. Chrysomphalus paulistus (Hempel) p, 504, 1900 .

Aspidiotus (Chrysomphalus) paulistus Hempel, Rev., IV,

Hab. Estado de S. Paulo, Ypiranga.

Mus. Paul. N. 14430, S. Paulo (cidade). Typo.

Em Laurus e outras plantas cultivadas e sylvestres.

151. Chrysomphalus porsonatus (Comstock)

Aspidiotus per sonatus Comstock, 2nd. Rep. Dep. Ent. Corn. Univ., p. 66, 1883.

Hab. Estado de S. Paulo, (São Paulo), Mexico, Indias Occidentaes, Guyana Ingleza, Inglaterra.

Em Eugenia jaboticaba, Citrus, Areca rubra, Sabal, Jasminum, Tillandsia corallina, Tillandsia confertiflora, Tillandsia saundersii, cajú, bdnaneira, figueira, mangueira.

\section{Chrysomphalus seutiformis (Cockerell)}

Aspidiotus scutiformis Cockerell, Ann. Mag. N. H. (6), XII, p. 481893.

Aspidiotus (Chrysomphalus) scutiformis Hempel, Rev., IV, p. $503,1900$.

Hab. Estado de S. Paulo, (S. Paulo). Estado de Minas Geraes, S. João d'el Rei, Mexico, America Central. 
Mus. Paul. N. 348, S. João del Rei (Est. Minas Geraes) Alv. da Silveira leg.; N. 15695̆, S. Paulo (cidade) Hempel leg. Em Laurus, Persea gratissima, Citrus.

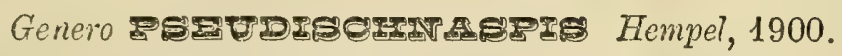

\section{Pseudisehmaspis linearis Hempel}

Pseudischnaspis linearis Hempel, Rev., IV, p. 506, 1900. Hab. Estado de S. Paulo, Ypiranga.

Mus. Paul. N. 330. Ypiranga, S. Paülo, Hempel leg. Typo.

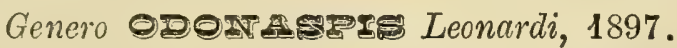

\section{Odonaspis janeirensis (Hempel)}

$500,1900$.

Aspidiotus (Odonaspis) janeirensis Hempel, Rev., IV, p.

Hab. Bahia do Rio de Janeiro, llha das Flores.

Mus. Paul. N. 15\%04, Ilha das Flores (Rio de Janeiro) Typo.

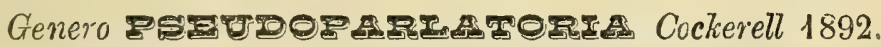

155. Iseudoparlatoria argentata n. sp. (*)

Hab. Estado de S. Paulo, Campinas.

Em murta cheirosa.

156. Pseudoparlatoria eristata (Lindinger)

Pseudoparlatoria cristata Lindg. Zeit. f. W. In. VII, (1), p. 10, 1911.

Hab. Japurá, Rio Negro.

Em Gnetum leyboldi.

157. Pseudoparlatoria noacki Cockerell. 1898.

Pseudoparlatoria noacki Ckll., Psyche VIII, p. 201,

Pseudoparlatoria noacki Ckll., Rev., III, p. 42, 1898.

Pseudoparlatoria noacki IIempel, Rev., IV, p. 511, 1900.

(*) Veja-se a descripção no fim dests catalogo. 
Hab. Estado de S. Paulo, Campinas.

Mus. Paul. N. 15707, Gampinas (Est. S. Paulo), Dr. F. Noack leg.

Em Nectandra.

\section{Pseandoparlatoria parlatorioides} (Comstock)

Aspidiotus? parlatorioides Comst., 2 Rep. Dep. Ent. Corn. Univ. p. 64, 1883.

Pseudoparlatoria parlatorioides Hempel, Rev. IV, p. $511,1900$.

Hab. Estado de S. Paulo, Ypiranga, S. Paulo, Cachoeira Estado do Rio de Janeiro, Nova Friburgo, Mexico, America do Norte.

Mus. Paul. N. 225 Novo Friburgo (Est. Rio de Janeiro); N. 245, Cachoeira (Est. de S. Paulo); N. 15708, Ypiranga, S. Paulo.

Em Psidium, Persea carolinensis, Drimys, Magnolia grandiflora, Oncidium varicosum, pecegueiro.

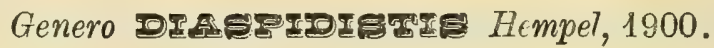

159. Diaspidistis multilobis Hempel.

Diaspidistis multilobis Hempel, Rev., IV, p. 522, 1900. Hab. Estado de S. Paulo, Ypiranga.

Mus. Paul. N. 15716, Ypiranga, S. Paulo, Hempel, leg. Typo.

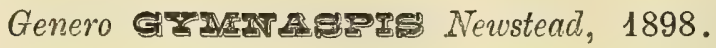

160. Gymnaspis aberemose Lindinger.

Gymnaspis aberemooe Lindinger, Deutsch. Ent. Zeit. IV p. $437,1910$.

Hab. Brazil.

Em Aberemoa rhizantha.

161. Gymmaspis aechmere Newstead.

Gymnaspis aechmece Newstead, Ent. Mon. Mag., XXXIV, p. 92, 1898.

Gymnaspis aechmece Hempel, Rev., IV, p. 508, 1900. 
Hab. Rio de Janeiro, Inglaterra.

IIus. Paul. N. 15705 , Rio de Janeiro, E. Ule leg. Em Aechmea aquilega.

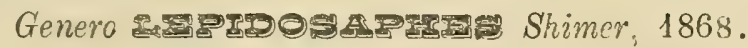

162. Tepidosaphes argentata (Cockerell)

Mytilaspis argentatus Ckll., Rev., II, p. 43, 1898. Mytilaspis argentatus Hempel. Rev., IV. p. 514, 1900. Hab. Estado de S. Paulo, Campinas. Mexico. Mus. Paul. N. 15711, Cachoeira.

Em uma arvore sylvestre.

\section{Lopidosaphos bambusicona (Cockerell)} 1899.

Mytilaspis bambusicola Ckll., Gan. Ent., XXXl, p. 44, IIytilaspis bambusicola Hempel, Rev., IV, p. 515, 1900. Hab. Estado de S. Paulo, Carnpinas.

Mus. Paul. N. 15710 Campinas (Est. S. Paulo) Dr. F. Noack leg.

Em bambú cultivado.

\section{Lepidosaphes beekii (Nerman).}

Coccus beckii Newman, The Ent., IV, p. 217, 1869. Iytilasp:s citricola Hempel, Rev., IV, p. 513, 1900.

Hab. Estado de S. Paulo, Campinas, S. Paulo, etc. Europa, Nadeira, Africa, Nauricia, Ceylão, Japão, Australia, Tasmania, Nova Zelandia. Fjji, Ilhas Hawai, Bermuda, Indias Ocvidentaes, America do Norte.

Irus. Faul. N. 1771: Campinas (Est. de S. Paulo) Dr. Noack leg.; N. 160ั42, Ypiranga, S. Paulo, Hempel leg.

Em Citrus, Ficus, C'roton, Elaeagnus, Banksia integrefolia, Taxus cuspidata, Cercidiphyllum japonıcum, Pomaderris apetala, Quercus.

\section{6อ̈. Lopidosaphes perlonga (Cockerell).}

Iylilaspis perlonga Ckil., Psyche, VIII, p. 202, 1898. Viytilaspis perlonga Ckll., Rev., II!, p. 42, 1898.

Mytilaspis perlonga IIempel, Rev., IV, p. 514, 1900. Hab. Estado de S. P'aulo, Ypiranga, Campinas. 
Mus. Paul. N. 262, Ypiranga, S. Paulo, Hempel leg.; N. 15709, Campinas (Est. S. Paulo) Noack leg.

Em Baccharis dracunculifolia.

\section{Lepidosapher ulmi (Linnceus).}

Coccus ulmi Linn., Syst. Nat., Ed. X. 1, p. 455, 1758. Mytilaspis ponorum Hempel, Rev., IV, p. 512. 1900.

Hab. Estado de S. Paulo. Em maçãs importadas da Republica Argentina. America do Norte, Europa, Algeria, Australia, Nova Zelandia, Japão, Ilhas Hawai.

Mus. Paul. N. 334 S. Paulo (cidade) Hempel leg.

Em Stillingia sebifera, Ailanthus glandulosus, Ceanothus anericanus, Sassafras officinale, Aesculus glabra, Syringa persica, Cysticus, Cormos, macieira, pereira, ameixeira, nogueira, roseira, carvalho, choupo, etc.

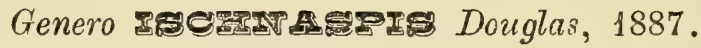

\section{Ischnaspis longirostris (Signoret).}

Mytilaspis longirostris Sign., Bull. Soc. Ent. Fr., (6), II, p. XXXV, 1882.

Ischnaspis longirostris Hempel, Rev., IV. p. 509, 1900.

Hab. Estado de S. Paulo, Campinas, Ypiranga, etc.; Inglaterra, Nova Zelandia, Australia, Japão, Panamá, Demerara, Indias Occidentaes, America do Norte. dade.

Mus. Paul. N. 15714, S. Paulo, (cidade); N. 169, Trin-

Em Latania, Pandanus, Magnolia grandiflora, Jasminum, Monstera, palmeiras, mangueiras, cafeeiros etc.

\section{Genero PS}

\section{Parlatoria perogandii (Comstock).}

Parlatoria pergandii Comstock, Rep. U. S. Dep. Ag., 1880, p. 327, 1881.

Parlatoria pergandii Hempel, Rev., IV, p. 510, 1900. Hab. Estado do Rio de Janeiro, Parahyba do Sul, America do Norte, Europa, Japão, Algeria, Ilhas Hawai.

Mus. Paul. 15706, Parahyba do Sul.

Em Citrus japonica. 
169. Parlatoria proteus (Curtis).

Aspidiotus proteus Curtis, Gard. Chron., p. 676, 1843.

Hab. Brazil, America do Norte, Europa, Australia, Japão, Ghina, Formosa, Ilhas Hawai.

Em Selenipedium, Macrozamia, Pinus insignis, Myrtus, Citrus, Camellia, Machilus, macieira, tamareira.

\section{DESCRIPÇÃO DE OITO ESPECIES NOVAS DE COCCIDAS}

Sub-FAMILIA MONOPHLEBINAE

cf. N. 5. Icorya genistae $n . s p$.

A femea adulta tem o corpo oval, um pouco mais largo na parte posterior do que na anterior. 0 dorso é coberto com uma secreção branca, disposta em cinco fileiras principaes, sendo uma central, duas sublateraes e duas marginaes. A secreção está disposta nestas fileiras em topetes irregulares e curtos, sendo os da fileira central mais compridos no centro e em cada extremidade. O ovi-sacco é grande e de forma conica, terminando, posteriormente, em um unico processo curto. Elle é composto de cera branca tingida côr de creme na base, tem o dorso marcado com cerca de 14 estrias longitudinaes, mas o lado inferior é liso. 0 insecto tem a largura de 3, 5 $\mathrm{mm}$., a altura de $2,75 \mathrm{~mm}$. e o comprimento de $6 \mathrm{~mm}$. sem o ovi-sacco, e de $13 \mathrm{~mm}$., incluindo o ovi-sacco.

Despido da secreção branca, o corpo tem a côr parda com a extremidade anterior amarellada. As antennas e as pernas são de côr pardo-escura, quasi pretas. Casido em uma solução de $\mathrm{KOH}$, elle torna o liquido turvo e de côr amarellada. O corpo, sem a secreção cerosa, tem o comprimento de $4,75 \mathrm{~mm}$., a largura de $2,50 \mathrm{~mm}$. e a altura de $2 \mathrm{~mm}$.

As antennas têm $0,909 \mathrm{~mm}$. a $1,0=8 \mathrm{~mm}$. de comprimento, e comprae-se de 11 articulaçбes. II bastante variação no comprimento das diversas articulaçðes, mas as proporçð̄es relativas são conservadas. A formula approximada é $11,(2,1$, 3) $(6,7,8,9,10),(4,5)$. O comprimento das articulaçбes, 
em micromillimitros, $\left({ }^{*}\right)$ é $\cdot(1), 105 ;(2), 105$ a $122 ;(3), 87$ a 105 ; (4), 61 a 70 ; (5), 61 a 70 ; (6), 70 a 79 ; (7), 70 a $79 ;(8), 70$ a $87 ;(9), 70$ a $87 ;(10), 70$ a $79 ;(11), 140$ a 175. Todas as articulaçōes tem pellos. Os olhos são grandes, escuros, conicos, e situados perto da base das antennas. As pernas são compridas e bem guarnecidas conı pellos; faltam os digitulos no tarso, que é curvado. As diversas articulações das pernas variam consideravelmente em comprimento, sendo estas as medidas das articulações do primeiro par de pernas. Coxa 210 a 227 ; femur com trochanter 542 a 612 ; tibia 525 a 612 ; tarso 192 a 297 ; unha 52 a 70 . Os digitulos da unha são filiformes e curtos.

Perto da margem posterior ha tres pares de espiraculos pequenos, cada um com um tubo curto e terminando em um botão, dando-lhe a forma de um cogumelo. No lado inferior do corpo e perto da extremidade posterior, ha ainda quatro orificios grandes, circulares ou ovaes, situados, tres, em linha transversal e perto da extremidade, e o outro, isolado, um pouco afastado e $\mathrm{em}$ frente dos outros. Es es orificios variam ex tamanho e tem até 100 micromillimetros de diametro.

Toda a superficie do corpo é guarnecido com numerosos pellos, sendo muit, delles curtos, mas outros ha que são fortes e compridos. Dispostos entre os pellos acham-se glandulas em grande numero, com orificios pequenos, circulares e compostos de 8 a 10 poros.

Os ovos são lisos, ellipticos, de côr parda, e têm cerca de $0,700 \mathrm{~mm}$. de comprimento e $0,262 \mathrm{~mm}$. de diametro transversal.

A larva recem-nascida tem a côr pardo-amarellada, com a extremidade das antennas escuras. o corpo é oval, medindo cerca de 0,ri00 mm. de comprimento e 0,330 $\mathrm{mm}$. de largura. As antennas têm seis articulaçð̋es, sendo a ultima a mais comprida. Os olhos são grandes e conicos. A margem lateral do corpo tem uma franja de pellos, sendo os seis pellos anaes muito compridos, e todos os outros muito mais curtos.

Eab. Campinas, no jardim do Instituto Agronomico, sobre Genista scoparia Lam, Lespedeza striata Hook \& A. e morango, Fragaria sp.

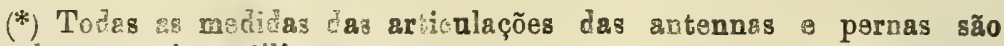
indicadas om micromillimetros. 


\section{Iceria genistae $n, s p$.}

The adult female oval in outline, a little wider posteriosly than anteriorly. The dorsum is covered with secretion, arranged in five principal rows, one central, two marginal. The secretior is arranged in short, irregular tufts, that are longer in the central row in the middle and at each extremity. The ovi-sac is large, conical in form, and terminates posteriorly in one shorl process. It is white with a creamy tint at the base, smooth on the ventral surface and finely striated with about 14 lines on the dorsum. The insect is $3,5 \mathrm{~mm}$. wide, $2,75 \mathrm{~mm}$. high, and $6 \mathrm{~mm}$. long without the ovi-sac, or $13 \mathrm{~mm}$. including the ovi-sac.

Denuled of wax, the body is brown with the anterior extremity yellowish. The antennae and legs are dark brown, nearly black. Boiled in a solution of $\mathrm{KOH}$, it turns the liquid turbid and yellow in color. The body is $4,75 \mathrm{~mm}$. long, 2,50 $\mathrm{mm}$. wide, and $2 \mathrm{~mm}$. high.

The antennae are from 0,90-1,058 mm. long, with eleven joints. The joints vary much in length, but their relative proportions are preserved. The length of the joints is: (1), $105 ;(\approx), 105$ to $122 ;(3), 87$ to $105 ;(4)$, til to $70 ;(5), 61$ to $70 ;(6), 70$ to $79 ;(7), 70$ to $79 ;(8), 70$ to $87 ;(9), 70$ to $87 ;(10), 70$ to $79 ;(11), 140$ to 175 . The approximate formula is $11,(2,1,3),(6,7,8,9,10),(4,5)$. All of the joints have hairs. The eyes are large, dark and conical in shape, and are situated near the base of the antennae. The legs arelong, well furnished with hairs, with the tarsus curved and without digitules. Length of the first pair of legs: coxa 210 to 2u7; femur with trochanter 542-612; tibia 525-612; tarsus 19\%-297; claw 52 to 70. The digitules of the claw are short and filiform.

Near the posterior margin of the body there are three pair of little spiracles, each one with a shorte tube terminating in a button, giving them the shape of mushrooms. On the ventral surface of the body, near the posterior exiremity, there are four large, circular or oval orifices, three of which are situater in a transverse row and near the extremity, and the remaining one is isolated, and placed a little in front of the others. These orifices vary in size, some of them having a diameter of 100 micromillimetrs.

The entire surface of the hody is furnished with haics, some of which are short, but there are others that are stout 
and long. Among the hairs there are numerous, circular glands, composed of 8 to 10 pores, and with small orifices.

The eggs are smooth, elliptical, brown in colcr, about $0,700 \mathrm{~mm}$. long and with a transverse diameter of $0,262 \mathrm{~mm}$. The newly hatched larva is yellowish brown in color with the ends of the antennae dark. The body is oval in outline, about $0,600 \mathrm{~mm}$, long and $0,330 \mathrm{~mm}$. wide. Antennae with six joints of which the last is the longest. The eyes are large and conical. The lateral margin of the body has a fringe of hairs, of which the six anal hairs are very long, and all of the others much shorter.

Hab. Campinas, in the garden of the Instituto Agronomico, on Genista scoparia Lam., Lespedeza striata Hook. \& A., and strawberry, Fragaria sp.

\section{SUB-FAMILIA DACTYLOPINAE}

\section{cf. N. 38. Tripersia taquarae n. $s p$.}

A femea adulta tem o corpo convexo, em iorma de coração, mais largo na extremidade anterior do que na posterior; com o comprimento de $5 \mathrm{~mm}$. e a largura maior de $4 \mathrm{~mm}$. Toda a superficie é coberta com um pó fino e branco.

Fervido em uma solução de $\mathrm{KOH}$, a derme conserva-se ainda um pouco dura, e o liquido tinge-se de côr pardo-clara.

As antennas medem $0,247 \mathrm{~mm}$. de comprimento e compõemse de 6 articulações, com os comprimentos seguintes: (1) 52 ; (2) $39 ;(3) 46 ;$ (4) 26 ; (5) $32 ;(6) 52$. A formula approximada é 16) 3254. As pernas são curtas e muito espaçadas umas das outras. O comprimento das articulações do primeiro par é o seguinte: coxa 72; femur e trochanter 286; tibia 97; tarso com unha 97. Os digitulos não foram observados. Nas pernas existem apenas poucos pellos curtos. $O$ annel anal tem 6 pellos. A derme é cheia de pequenas glandulas circulares, e tambem tem numerosos espinhos dispostos em carreiras transversaes.

As larvas recem-nascidas têm o corpo de forma elliptica, de côr pardo-clara, com os olhos pretos. O abdomen tem os segmentos distinctos, cada um com um pello curto na margem. O corpo tem, na sua extremidado posterior, dcis pellos muito compridos, e outros pellos curtos na extremidade anterior entre as antennas. As antennas comprem-se de 6 articulações 
e tambem são guarnecidas com muitos pellos. 0 rostro é grande e o laço rostral alcança a extremidade posterior do corpo. As pernas são curtas e guarnecidas de pellos.

Hab. Cantareira. Estado de S. Paulo. No interior do taquara-uçú e acompanhado por formigas. Existe na collecção do Museu Paulista soh o n. 15.630. Enviado pelo Director do Museu Paulista, Dr. H. v. thering.

\section{Ripersia taquarae $n . s p$.}

The adult female has the body convex, heart shaped, wider at the anterior extremity than at the posterior, having a total length of $5 \mathrm{~mm}$. and a width of $4 \mathrm{~mm}$. The entire surface is covered with a fine, white powder.

Boiled in a solution of $\mathrm{KOH}$, the liquid is colored light brown, and the derm remaines somewhat hard.

The antennae are composed of 6 joints and are $0,247 \mathrm{~mm}$. long. The various joints have the following lengths: (1) 52 ; (2) 39 ; (3) 16 ; (4) $\therefore 6$; (5) 32 ; (6) 52. The approximate formula is (16) 3254 . There are three pairs of short legs set very wide apart. The joints of the first pair of legs have the following lengths: coxa 72; femur with trochanter 286; tibia 97; tarsus and claw 97. No digitules were observed, and the legs have but few short hairs. The analring is furnished with 6 large hairs. The derm contains innumerable small circular glands, and many spines arranged in transverse lines.

The newly hatched larvae have elliptical, light brown bodies with black eyes. The abdomen is composed of distinct segments each one with a short marginal hair on each side. The body has two very long hairs at the posterior extremity and several shorter hairs at the anterior extremity between the antennae. The antennae are conposed of 6 joints all of which are furnished with many long hairs. The legs are short and furnished with hairs. Rostrum large and rostral loop reaches to the posterior extremity of the body.

Hab. Cantareira. State of S. Paulo. On the interior of large cane accompanied by ants. N. 15.630 in the collection of the Museu Paulista. Sent by the Director of the Museu Paulista, Dr. H. v. Ihering. 


\section{Stb-Familia COCCINAE}

\section{(f. N. 115. Saissetia lucida $n . s p$.}

A femea adulta tem o corpo oval, um pouco achatado, com 9,5 $\mathrm{mm}$. de comprimento, $6,5 \mathrm{~mm}$. de largura e $3,5 \mathrm{~mm}$. de altura. A côr do insecto no dorso é pardo-escura, com uma fita marginal preta. A derme do dorso é enrugada, reluzente e dura. Removido da casca da arvore, o insecto deixa uma fina camada de cera.

Fervido em uma sulução de $\mathrm{KOH}$, elle tinge o liquido de côr pardo-clara. A derme amolece e torna-se transparente, mas conserva-se grossa. O dorso está cheio de grandes glandulas redondas ou irregulares, as quaes se desprendam pela ebulição. As glandulas na fita marginal são mais persistentes que na outra parte da derme.

As antenas compõe-se de 8 articulações, e tem cerca de 0,50 óm de comprimento. O comprimento das articulações é o seguinte: (1) 71; (2) 65; (3) 91;:4) 78; (5) 91; (6) 42; (7) 36; (8) 52. A formula approximada é (35) 412867. Todas as articulações têm pellos. Existem tres pares de pernas bem desenvolvidas. As articulações do primeiro par de pernas tem 0 seguinte comprimento: coxa 117; femur e trochanter 305; tibia 195; tarso 130; unha 26. Os digitulcs do tarso são delgados e compridos, e os da unha largos, com a extremidade muito dilatada. Ao redor da margem lateral do corpo ha uma fileira de espinhos finos e compridos. Os espinhos da região dos espiraculos são em numero de 3 , e são mais grossos que os outros.

Eab. Alto da Serra. Estado de S. Paulo. Na casca de uma arvore sylvestre. Tem o n. 15534 na collecção do Museu Paulista. Enviado pelo Director do Museu Paulista Dr. H. v. Ihering.

\section{Säassetia Iucida $n$. $s p$.}

The body of the adult female is oval in form, slightly flattened, and is $9,5 \mathrm{~mm}$. long, $6,5 \mathrm{~mm}$. wide and $3,5 \mathrm{~mm}$. high. The color of the dorsum is dark brown, with a narrow, black marginal band. The derm is wrinkled, hard and shiney, when removed from the bark the insect leaves a thin waxy scale. 
Boiled in a solution of $\mathrm{KOH}$, the liquid is tinged light brown. The derm becomes soft and transparent, but remains thick. The dorsum is full of large round or irregular glands which are loosened and fall out by the boiling. The glands of the marginal band are more persistent than those on the other part of the derm.

The antennae are composed of 8 joints, and are. $0,525 \mathrm{~mm}$. long. The individual joints have the following lengths: (1) 71; (2) 65 ; (3) 91 ; (4) 78 ; (5) 91 ; (6) 42; (7) 36 ; (8) 52. The approximate formula is (35) 412867. All of the joints bear hairs. The joints of the first pair of legs have the following lengths: coxa 117; femur with trochanter 305; tibia 195; tarsus 130; claw 26. The digitules of the tarsus are fine and long, those of the claw wide, with the distal ends widely expanded.

The body is furnished with a marginal row of fine, long spines. The spines of the spiracular regions are three in number and are stouter than the others.

Hab. Alto da Serra. State of S. Paulo. On the bark of a forest tree. Present in the collection of the Museu Paulista as N. 15534. Sent by the Director of the Museu Paulista, Dr. H. v. Ihering.

\section{cf. N. 51. Pulvinaria ormata $n . s p$.}

A femea adulta tem o corpo hemispherico, com o lado ventral chato e oval, sendo elle um pouco mais largo na parte posterior do que na anterior. O dorso é duro e enrugado. A côr geral é creme no lado dorsal, e avermelhada no lado ventral. As rugas do dorso têm a côr pardo-escura, e ha tambem uma faixa mediana o longitudinal de côr amarello-clara. Esta faixa principia perto da margem anterior do corpo e extende-se até chegar quasi a tocar as placas anaes. Estas placas são pequenas e triangulares e de côr parda. Todo o insecto é coberto com uma carnada fina de cera branca, e quando se o tira do galho, tambem deixa uma fina escama de cera branca. Os individuos maiores tem $7 \mathrm{~mm}$. de comprimento, $4,25 \mathrm{~mm}$. de largura, e $3,25 \mathrm{~mm}$. de altura.

0 ovi-sacco é estreito, tendo apenas 2,50 a $3 \mathrm{~mm}$. de largura, chato, mas muito comprido, alcançando, geralmente, o comprimento de $6 \mathrm{~cm}$., mas ha individuos com o ovi-sacco de 1i) $\mathrm{cm}$. de comprimento. A secreção do ovi-sacco é branca, 
e muito adherente. Os ovos são pequenos, de forma oval, reluzentes e côr de carne.

As antennas são curtas, com seis articulações apenas. As vezes, a sexta articulação, que termina em um grupo de pellos, está dividida formando uma articulação falsa. A primeira e a terceira articulação são as mais compridas, sendo a quarta a mais curta. A formula approximada é 315624 ou (:31) 5 (62) 4. O seu comprimento total é de $0,486 \mathrm{~mm}$. a $0,435 \mathrm{~mm}$., tendo as articulações os comprimentos seguintes: (1), 91 a 97; (2), 65; (3), 97; (4), 39 а 52; (5), 78 а 97; (6), 65 а 78.

As pernas são curtas e grossas, sendo o tarso rnuito curvado. Tanto o tarso como a unha tem os digitulos delgados com as suas extremidades dilatadas. As articulações do primeiro par de pernas tem o comprimento seguinte: coxa 157, femur com trochanter 5:55, tibia 332 a 350, tarso 157, unha 5?. 0 mento é relativamente pequeno.

A margem lateral do corpo é guarnecida com uma carreira dupla de pequenos espinhos. Ha tres espinhos grossos na região dos espiraculos, sendo um comprido e os dois outros muito curtos. 0 annel anal tem 10 pellos compridos. Toda a superficie da derme é provida de numerosas glandulas pequenas, tanto circulares como compridas, e tambem tem alguns espinhos curtos.

O macho é grande, de côr pardo escura com as antennas e as pernas amarelladas. As antennas tem 0,787 $\mathrm{mm}$. de comprimento e compõe-se de nove articulações, das quaes as primeiras são as mais curtas. As azas são largas, transparentes e ornamentadas com pellos diminutos. 0 corpo, inclusive 0 estylete, tem $1.138 \mathrm{~mm}$. de comprimento.

Hab. Campinas. Jardim do Instituto Agronomico, nos galhos de uma planta trepadeira, Arrabidaea sp. da familia Bignonaceae.

\section{Pulvinaria ornata $n$. $s p$.}

The adult female has the body hemispherical in form, with the ventral surface flat and oval in outline, a little wider posteriorly than anteriorly. The dorsum is hard and wrinkled. The general color is cream on the dorsal surface, and with a reddish tint on the ventral surface. The wrinkles on the dorsum are dark brown in color, and there is also a longitudinal median band, yellow in color, and extending to the anal plates, wich are small. triangular, and brown in color. The entire insect is covered with a fine layer of white wax, and when the insect is 
removed from the twig, it leaves a fine scale of white wax. The largest individuals are $7 \mathrm{~mm}$. long., $4,25 \mathrm{~mm}$. wide, and $3,25 \mathrm{~mm}$. high.

The ovi-sac is narrow, from $2,50 \mathrm{~mm}$. to $3 \mathrm{~mm}$. wide, flat, and from 6 to $10 \mathrm{~cm}$. long. The secretion of the ovi-sac is white and very sticky. The eggs are small, oval, shiney, and flesh colored.

The antennae are short, six-jointed. Sometimes the last joint, which ends in a brush of hairs, is divided, making a false joint. The length of the antennae is $0,486 \mathrm{~mm}$. to $0.435 \mathrm{~mm}$. , and that of the joints is : (1), 91 to 97 ; (2), 65; (3), 97 ; (4), 39 to 52 ; $(5), 78$ to $97 ;(6), 65$ to 78 . The approximale formula is 315624 or (31) 5 (62) 4 .

The legs are short and thick, the joints of the first pair measuring: coxa 157, femur and trochanter 525, tibia 332 to 350, tarsus 157, claw 52. The digitules of tarsus and claw are fine with the distal end expanded. The mentum is relatively small.

The lateral margin of the body is furnished with a double row of short spines. The region of the spiracles is characterized by three thick spines, one of which is long and the two remaining ones are short. The anal ring has 10 long hairs. The entire surface of the derm is furnished with numerous glands, some circular and some long, but all small; and also contains some small, short spines.

The male is large, dark brown in color, whith the antennae and legs yellow. The antennae are $0,787 \mathrm{~mm}$. long, and are composed of nine joints, of which the first are the shortest. The wings are wide, transparent, and ornamented with minute hairs. The body, including the style, is $1.138 \mathrm{~mm}$. long.

Hab. Campinas, State of S. Paulo. In the garden of the Instituto Agronomico, on the twigs of a climbing plant, Arrabidaea sp. of the family Bignonaceae.

\section{D̃UB-FAMILIA DIASPINAE}

\section{cf. N. 155. Pseudoparlatoria argentata $n$. $s p$.}

Escudo da femea adulta circular até subcircular, um pouco achatado na margem anterior, com cerca de 1,226 $\mathrm{mm}$. de diametro. O escudo é duro, chato, de côr preta, com um circulo excentrico e uma area marginal transparente. As pel- 
liculas são marginaes, sendo a primeira pequena e de côr amarello-clara, e a segunda muito grande e transparente na margem, ficando o restante da pellicula coristituido em uma mancha triangular e preta. O escudo do macho tem a forma um pouco mais alongada do que o da femea, e é tambem menor, pois alcança apenas 0,612 a 0,70 ) $\mathrm{mm}$. no seu diametro maior. Este escudo não é preto, pois destaca-se bem a pellicula amarella na parte mais elevada, que é marginada por uma area quasi transparente.

A femea adulta é subcircular, tendo a maioria dos individuos apenas $0,525 \mathrm{~mm}$. de diametro, mas os individuos alcançam o diametro de $0,717 \mathrm{~mm}$. Ella tem a côr amarelloclara ou branca. O pygidiuin tem tres pares de lobulos, todos muito curtos, e com a margem posterior arredondada. 0 par mediano tem os lobulos largos e simples, sendo o segundo e terceiro par de lobulos profundamente incisos. Entre os dois lobulos medianos ha um par de placas simples; entre estes e os segundos ha uma placa que tem a extremidade posterior incisa; e entre os segundos e os terceiros lobulos ha uma placa simples. A margem do pygidium, na parte anterior dos lobulos, é espessa, dura e finamente dentada. Os tres segmentos do corpo anterior do pygidium tèm a margem lateral modificada em lobulos grandes. Existem no pygidium nume. rosas glandılas circulares, e algumas transversaes e outras lineares; os antero-lateraes variam de 12 a 19 , os posterolateraes de 14 a 25̆. e o grupo mediano de 1 a 4. Este grupo mediano falta em alguns exemplares.

O macho é pequeno, de côr pardo-escura, com as antennas e as pernas mais claras. O corpo, inclusive o estylete, tem 0,612 mm. de comprimento e 0,210 mm. de largura. Os olhos são grandes e de côr preta. As antennas têm dez articulações. As azas medem $0,647 \mathrm{~mm}$. de comprimento e são transparentes.

As larvas recem-nascidas tem o corpo elliptico, chato, e de côr branco-am rellada, com dois pelios curtos na extremidade posterior. Os olhos não são visiveis. Comprimento do corpo $0,245 \mathrm{~mm}$. largura $0,131 \mathrm{~mm}$.

Os ovos são brancos, ellipticos, lisos e reluzentes, e têm 0,210 $\mathrm{mm}$. de comprimento e 0,105 mm. de largura. Cada femea pre de 30 a 35 ovos.

Hab. Campinas, em folhas de murta cheirosa, Aglaia sp. da familia Meliaceae. 
Os escudos das femeas e dos machos, agrupados no lado inferior das folhas, são cobertos com uma camada muito fina de secreção branca, que dá á folha a apparencia de ser prateada.

\section{Pseudoparlatoria argentata $n . s p$.}

The female scale is circular to subcircular in outline, slightly flattened at the anterior margin, and about $1.226 \mathrm{~mm}$ in diameter. The scale is hard, flat, black, with an excentric circle and a marginal area transparent. The pellicles are marginal ; the first is small and light yellow in color, the second is large, with a transparent margin, and the remainder forming a black, triangular spot. The male scale is slightly smaller than that of the female, and also more elongate, being from 0,612 to $0,700 \mathrm{~mm}$. long. This scale is not black, but consists of the yellow pellicle situated in the central elevated area and the marginal area which is nearly transparent.

The adult female is subcircular in outline, having a diameter of about $0,525 \mathrm{~mm}$. but there are some individuals that have a diameter of $0,717 \mathrm{~mm}$. The body is light yellow in color or white, with the posterior margin rounded, having three pair of very short lobes on the pygidium, of which the median pair is wide and simple, and the second and third pairs are deeply incised. Between the two median lobes there is one pair of simple plates; between this and the second pair there is a plate that is incised; and between the second and third pair there is a simple plate. The margin of the pygidium cephalad of the lobes, is thick hard and finely dentated. The three segments of the body cephalad of the pygidium, have the lateral margin modified into large lobes.

The pygidium contains numerous circular glands, others transverse, and still others linear. There are usually five groups of circumgenital glands; of which the anterior-laterals vary from 12 to 19 , the posterior-laterals from 14 to 25 , and the median group from 1 to 4 . In some specimens the median group is wanting.

The male is small, dark brow in color, with the antennae and the legs lighter. The body is $0,210 \mathrm{~mm}$. wide and $0,612 \mathrm{~mm}$. long, including the style. The eyes are large and black. The antennae have ten joints. The wings are 0,647 $\mathrm{mm}$. long and are transparent.

The newly hatched larvae have the body elliptical, fat, and yellowish white in color, with two short terminal hairs. 
The eyes are not apparent. The body is $0,245 \mathrm{~mm}$. long and $U, 131 \mathrm{~mm}$. wide.

The eggs are white, elliptical, smouth and shiney, and are $0,210 \mathrm{~mm}$. long and $0,105 \mathrm{~mm}$. wide. Each female lays from 30 to 35 eggs.

Hab. Campinas, State of S. Paulo. On leaves of «fragrant «, Aglaia sp. of the family Meliaceae.

The scales of both males and females are grouped on the under side of the leaves and are covered with a very fine white secretion, giving them a silvery appearance.

\section{Ceroplastes excaecariae $n . s p$.}

0 escudo de cera da femea méde $5 \mathrm{~mm}$. de comprimento, $5,5 \mathrm{~mm}$. de largura e $2 \mathrm{~mm}$. de altura; é irregular, branco corn colorido amarello, e não é dividido em placas distinctas. A cêra é dura, mas sem ser quebradiça, profundamente recortada nos nucleos, especialmente no dorsal; este é grande e de côr parda, escura. Ha 8 nucleos, sendo um dorsal, um anterior, 2 posteriores e 2 lateraes em

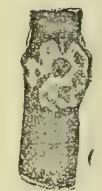
cada lado. Duas linhas de giz partem dos nucleos lateraes e chegam até embaixo á face ventral. O corno caudal termina em ponta glabra. Especimens mais novos têm cera mais escura; nos mais velhos a cera está quebrada e é muito convexa. Despida da cera, a femea tem configuração oval, é chata e de cor castanho-escura, medindo $3,5 \mathrm{~mm}$. de comprimento, $3 \mathrm{~mm}$. de largura e $1,35 \mathrm{~mm}$. de altura. Ha um pequeno lobulo anterior, 3 lobulos lateraes de cada lado e um dorsal maior que os outros. O corno caudal, que é inserido verticalmente, é muito curto.

Cosido em solução de KOH, a derme torna-se transparente e macia.

Antennas e pés são curtos. As antennas, que se compõem de 7 articulos, medem (1) 46; (2) 39; (3) 97; (4) 33; (气) 33 ; (6) 20 ; (7) 20 ; a formula approximada é 312 (40) (67). Comprimento do primeiro par de pernas: coxa, 65-71; trochanter e femur, 117-123; tibia 65-84; tarso 39-45; unha 13. O corno caudal é bruno escuro. A areas estigmaes são caracterizadas por 40 a 50 glandulas claviformes, assim como por numerosas pequenas fieiras circulares, que se extendem atẻ os espiraculos. 
Parece ser muito alliada a $C$. iheringï Ckll. e C. scutigera Ckll.

Hab. Ypiranga, Estado de S. Paulo.

Sobre Excaecaria biglandulosa M. Arg.

Ceroplastes excaecariae $n . s p$.

Waxy scale of female $5 \mathrm{~mm}$. long, $5,5 \mathrm{~mm}$. wide, and $2 \mathrm{~mm}$. high; irregular, white with yellowish tinge, not divided into distinct plates. Wax hard but not brittle, deeply indented at nuclei, especially the dorsal one; which is large and dark brown in color. Eight nuclei present, being one dorsal, one anterior, two posterior, and two lateral on each side. Two chalky lines from the lateral nuclei reach under the ventral surface. End of caudal horn bare. Younger specimens with wax darker; older specimens with wax broken and very convex. Denuded of wax, the female is oval in outline and flat, of a dark chestnut color, being $3,5 \mathrm{~mm}$. long, $3 \mathrm{~mm}$. wide, and $1,35 \mathrm{~mm}$. high. There is one small anterior lobe, three lateral lobes on each side, and one dorsal larger than the others. The caudal horn is very short and inserted vertically.

Boiled in a solution of $\mathrm{KOH}$, the derm becomes transparent and soft. Antennae and legs short. Length of antennae, that are sevenjointed, 0,288 to $0,318 \mathrm{~mm}$. Length of joints, (1) 46 ; (2) 39 ; (3) 97 ; (4) 33 ; (5) 33 ; (6) 20 ; (7) 20. Approximate tormula 312 (45) (67). Lenght of the first pair of leg, coxa, 65-71; trochanter and femur, 117-123; tibia 65-84; tarsus 39-45; claw 13. Caudal horn dark brown. Stigmatal areas characterized by 40 to 45 club-shaped glands, and many small circular spinnerets, which extend to the spiracles. tigera Ckll.

Apparently closely related to C. iheringi Gkll. and C. scuHab. Ypiranga, State of S. Paulo.

On Excaecaria biglandulosa M. Arg.

\section{Mesolecanium Iucidum $n . s p$.}

A forma adulta tern forma globular e méde $6,25 \mathrm{~mm}$. de comprimento, $7,25 \mathrm{~mm}$. de largura e 5,50 de altura. A. derme é dura, lisa relusente e de cor bruno-castanha, com colorido amarello nos lados; na margem ventral é de côr 
mais escura A margem lateral é provida de lobulo posterior e nas areas estigmaes. Ciosido em solução de $\mathrm{KOH}$, o liquido toma cor bruno-clara, ao passo que a derme se torna quasi transparente e flexivel.

As antennas médem 0,511 a $0,530 \mathrm{~mm}$. de comprimento e compõem-se de 8 articulos, que tem as seguintes dimensões : (1) 78 ; (2) 91 ; (3) 91 ; (4). 45; (5) 58 ; (6) 45; (7) 15; (8) 58. A formula approximada é (23) 1 (58) 467.

Comprimento dos articulos do primeiro par de pernas: cocha 143; trochanter e femur 292; tibia 201; tarso 123 ; unha 19. Os digitulos das unhas bem como a derme contem numerosas pequenas glandulas redondas. As areas stigmaes são muito recortadas e caracterizadas por 3 espinhos grossos; um longo e os dous outros mais curtos; além disto numerosas pequenas glandulas circulares.

Hab. Porto Alegre, Estado do Rio Grande do Sul.

Sobre uma Solanacea.

\section{Mesolecanium lucidum $n . s p$.}

Adult female, globular in shape, 6,25 mm. long, 7,25 mm. wide, and 5,50 mm. high. Derm hard, smooth, shiney chestnut brown in color, with a yellow tint at sides. and the ventral margin darker. Lateral margin lobed posteriorly and at the stigmatal areas. Anal fissure $2 \mathrm{~mm}$. long, with the sides contiguous. Boiled in a solution of $\mathrm{KOH}$, it turns the liquid light brown; the derm itself becomes nearly transparent and flexible.

The antennae are $0,511 \mathrm{~mm}$. to $0,530 \mathrm{~mm}$. long and are composed of eight joints with the following dimensions; (1) 78 ; (2) 91 ; (3) 91 ; (4) 45 ; (5) 58 ; (6) $45:(7) 45$; (8) 58. Approximate formula (23) 1 (58) 467. Length of the joints of the first pair of legs : coxa 143 ; trochanter and femur 292; tibia 201; tarsus 123; claw 19. Digitules of claw as well as derm contains numerous small, round glands. Stigmatal areas are much indented and characterized by three thick spines, one long and the other two shorter, and by numerous small circular glands.

$\mathrm{H} a b$. Porto Alegre, State of Rio Grande ao Sul.

On a Solanaceae.

Megasaissetia brasiliensis $n . s p$.

A femea adulta méde $10 \mathrm{~mm}$. de comprimento, $8,5 \mathrm{~mm}$. de largura, e $7 \mathrm{~mm}$. de altura; é de cor bruno- castanha, clara. 
O escudo é muito convexo, sub-globular, e com a face ventral ligeiramente deprimida. A derme é dura, lisa e relusente; torna-se mais macia depois de cosida em solução de $\mathrm{KOH}$, comtudo é bastante chitionosa para manter a sua forma. A derme tem os polygonos peculiares ao genero Saissetia. A fissura anal méde $1,5 \mathrm{~mm}$. e tern os bordos contiguos.

As peruas e antennas são normaes, estas ultimas medindo $0,569 \mathrm{~mm}$. de comprimento e são compostas de 8 articulos, com as seguintes medidas: (1) 71 ; (2) 71 ; (3) 97 ; (1) 78; (5) 97 ; (6) 45 ; (7) 45 ; (8) 65 ; formula approximada $(35)$ 4 (12) $S(67)$. Comprimento dos articulos do primeiro par de pernas: coxa 130; femur e trochanter 318; tibia 227; tarso 130; unhas 26. Os digitulos do tarso são longos, com a extremidade distal alargada; os da unha são mais curtos e largos. O bordo marginal é provido de tuberculos espinosos, longos e grossos. As areas estigmaes são caracterizadas por tres espinhos em cada uma, sendo um longo e curvado e os outros dous menores.

Hab. Parque Gajurú, Alto da Serra, Estado de S. Paulo.

\section{Megasaissetia bragiliensis $n . s p$.}

Adult female, $10 \mathrm{~mm}$. long, $8,5 \mathrm{~mm}$. wide, and $7 \mathrm{~mm}$. high, of a light chestnut brown color. The scale is very convex, sub-globular, with the ventral margin slightly depressed. The derm is hard, smooth and shiny, becoming softer after boiling in a solution of $\mathrm{KOH}$, but being chitinized enough to retain its furm. Derm with the polygons peculiar to the genus Saissetia. Anal fissure $1,5 \mathrm{~mm}$. long, with the sides contiguous.

Legs and antennae normal, the latter 0,569 $\mathrm{mm}$. long, and composed of 8 joints, with the following measurements : (1) 71 ; (2) 71 ; (3) 97 ; (4) 78; (5) 97 ; (6) 45 ; (7) 45 ; (8) 55. Apprcximate formula, (35) 4 (12) 8 (67). Length of the joints of the first pair of legs, coxa 130; femur and trochanter 318 ; tibia 227; tarsus 130; claw 26. Digitules of tarsus long, with the distal end expanded; those of the claw shorter and broader. There is a marginal fringe of rather long, stout, tuberculate spines. The stigmatal areas are characterized by three spines each, one large and curved, and the other two smaller.

Hab. Parque Cajurú, Alto da Serra, State of S. Paulo. Campinas, 26 de Fevereiro de 19.12. 



\section{INDICE ALPHABETICO}

Pags.

abremore (Gymuspis). . . . . . . . . 52 aechmeas (Gymnaspis). . . . . . . . . . 52 $\Delta$ kgrmes . . . . . . . . . . . . . 39 albata (Tectopulvinaria) • . . . . . . . . 29 albolinestus (Ceroplastes) . . . . . . . . . 29 Alıchtensia . • . . . . . . . . . 35 amszonicus (Ceroplsstes) • . . • • . . . 30 aonidum (Chrysomphalus) (Aspidiotus) . . . . 49 acnidum (Chrysomphalus) . . . . . . . . . 49 aonidum (Coccus) . . . . . . . . . . . 49

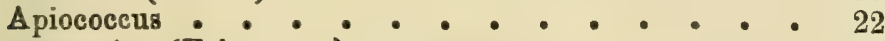
araucariae (Eriococeus) • • • • • • • . 21 arecae dictyospermi (Chrysomphalus) . . . . 5 J argentat 1 (Lepidosaphes) . . . . . . . . . 53 argentata (Lichtonsia) • • • • • • • • • . 29 argentata (Pseudoparlatoria) 51, . . . . . . 63 argentatus (Mytilaspis). • • • • . . • . 53 armatum (Exium) - • • • . . . . . . . 25 armatus (Eriococcus) • • • . • • . . . 25 articulatus (Aspidiotus) • • • • • • • • . 49 articulatus (Selenaspidus) . . . . . . . . . 41 ssper (Stigmacoccus) • . • • • • • • . 17 ssperatus (Apiococcus) . . . . . . . . . 22 aspidistrae (Chionaspis) . . . . . . . . . 44 aspidistrae (Hemichionaspis) . . . . . . . . 44 Aspidiotus . . . . . . . . . . . . 46 Asterodiaspis . . . . . . . . . . . 20 Asterolecanium . . . . . . . . . . . . 19 attonusta (Alichtensia). • . . . . . . . . 35 attenuata (Lictensia ?). . . . . . . . . . 35 Aulacaspis . • • • . • • • • • • . . 44 sustralis (Diaspis) . . . . . . . . . . 43 baccharidis (Lscanium). • • • • • • . . 37 bsccharides (Mesolecanium) . • • • • . . 37 baccharidis (Bolenceocus). • • • • • . . 20 bambusae (Asterolecanium) • . • . . . . . 19 bambusae (Chretococeus) . . . • . . . . . 25 bambusae (Shpaerococcus) • • • • • • • . . 25 
Pag.

bambusicola (Lepidosaphes) . . . . . . . 53

bambusicola (Mytilaspis) . . . . . . • . . 53

beckii (Coccus) - . . . . . . . . . . 53

beckii (Lepidosaphes) . . . . . . . . . . 53

bicolor (Ceroplastes) . . . . . . . . . 30

biclavis (Chionaspis) • . . . . . . . . . 43

biclavis (Howardia). . . . . . . . . . 43

boisduvalii (Aulacaspis) . . . . . . . . . 43

boisdovalii (Diaspis) . . . . . . . . . . 43

bcisduvalii maculata (Aulacaspis) . . . . . . 43

boiscuvalii msculata (Diaspis). . . . . . . . 43

brasiliensia (Cryptokgrmes). . . . . . . . 23

brasiliensis (Eriococeas) . . . . . . . . . 21

brasiliensis (Icerys). . . . . . . . . . 18

brasiliensis (Megasaissetis) 68 • . . . . . . 69

brasiliensis (Palaeococcus). • . . . . . . 17

brevicornis (Termitococcus) • . . . . . . 26

bromeliae (Coccus) . . . . . . . . . . . 44

bromeliae (Diaspis) . . . . . . . . . . . 44

bromeliae (Pseudococcus) . . . . . . . . . 24

brunfelsize (Eucalymnstus) . . . . . . . . 36

brunfelsiae (Lecanium). . . . . . . . . . 36

buxi (Aspidiotus) . . . . . . . . . . . 45

buxi (Pinoaspis). . . . . . . . . . . 45

cacti (Diaspis) . . . . . . . . . . . . 44

cserules Tachardia . . . . . . . . . . 26

camelliae (Aspidiotus) . . . . . . . . . . 48

campinensis (Ceroplsstes) . . . . . . . . . 30

campomanesiae (Lecaniun) . . . . . . . . 37

campomanesiae (Mesolecanium ?). . . . . . . 37

Capulinis . . . . . . . . . . . . 23

Carpochloroides . • . . . . . . . . . . 22

cassir (Ceroplastes). . . . . . . . . . 30

cassize (Coccus). • . . . . . . . . . . 30

cecropia (Lachnodiella) . . . . . . . . . 24

Ceroplsstes . . . . . . . . . . . . . 29

Chatococcus. . . . . . . . . . . . . 25

Chionsspis . . . . . . . . . . . . 42

Chrysomphalas . . . . . . . . . . . . 49

citri (Chionaspis) . . . . . . . . . . . 42

citricola (Mytilaspis) . . . . . . . . . . 53

citri (Dactylopius) . . . . . . . . . . . 24

citri (Dorthesia). . . . . . . . . . . . 24

citri (Pseudococcus) . . . . . . . . . . 24

Cocoidas . . . . . . . . . . . . . . 17

Coccinae . . . . . . . . . . . . . 27

Coccus . . . . . . . . . . . 36

communis (Ceroplastes). . . . . . . . . 30

Conehaspinae . . . . . . . . . . . 19

Conchaspis . . . . . . . . . . . . 19 
confluens (Ceroplastes) . . . . . . . . 30

convexa (Protopulvinaria) - . . . . . . . 28

cordise (Disspis). . • . . . . . . . . . 44

crateraformans (Capulinia). . . . . . . . . 23

crateraformis (Cspulinia) . . . . . . . . . 23

eristata (Esendoparlatoris). . . . . . . . . 51

Cryptokermes . . . . . . . . . . . 23

Crypticerya . . . . . . . . . . . . . 17

culcus (Ceroplastes). • • . . . . . . . . 31

caneatua (Ceroplastes) . . . . . . . . . . 31

cyonophylli (Aspidiotus) • . . • • . • . . 46

cydonime (Aspidiotus) • • • • . • • • • . 46

cydonize (Tachardis) • . . . . . . . . . 26

Dactylopinঞ。 . . . . . . . . . . . . 19

Dactylopius . . . . . . . . . . . . . 24

depressa (Pulvinaria) • . . . . . . . . . 27

deprersa (Saissetia). . . . . . . . . . . 40

depressum (Lecanium) . . . . . . . . . . 40

destructor (Aspidiotus). . . . . . . . . . 42

Diaspidistis . . . . . . . . . . . . . 52

Diaspinae. • . . . . . . . . . . . 42

Disspis . . . • . . . . . . . . 43

dictyospermi arecae (Aspidiotus) . . . . . . 50

dictyospermi arecse (Chrysomphalus). . . . . . 50

dictiospermi (Aspidiotus) • • • • • • • . . 49

dictyospermi (Chrys зmphalus). . . . . . . 49

discoides (Lecanium) . . . . . . . . . 40

discoides (Saissetia) . . . . . . . . 40

Dorthesia. . . . . . . . . . . . . . 24

dura (Srissetia) . . . . . . . . . . 40

durum (Lecanium) . . . • . . . • . . 40

echinocacti cacti (Diaspis). . . . . . . . . 44

erythrinae (Lecsnium) • . . . . . . . . . 42

Eucalymnatas • • • • • • • • • 36

eugeniae (Eulecanium) • . . . . . . . . . 40

eugeniae (Lecanium) . . • . . . . . 40

eugeniae (Pulvinaria) . . . . . . . . . . 27

Ealecanium - . . . . . . . . . . 40

excaecariae (Ceroplastes), 33 e . . . . . 66

Edwallia . . . . . . . . . . . . 35

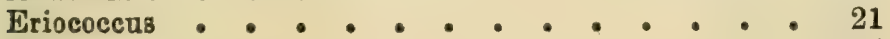

Erium. . . . . . . . . . . . 25

fairmsirii (Ceroplasıs). . . . . . . . . 31

ficus (Palvinaria) • . . . . . . . . 28

Fioriais . . . . . . . . . . . 46

fioriniae (Diaspis) • • • • • • • • • 46

fioriniae (Fiorinis) . . . . . . • • 446

floridensis (Ceroplastes) . . . . . . . . 31

fluminensis (Conchaspis) . . . . . . . . 19

farmicarius (Ceroplastes) . . . . . . . 31 
Pags.

formosus (Ceroplastes) - . . . . . . . 32

genistre (Icerya), 18 ๑. . . . . . . . . 55

glanulosum (Lecanium) . . . . . . . . . 41

glinuloea (Saissetia) . . . . . . . . . 41

globosus (Apiococcus) . . . . . . . . . . 22

gracile (Lecanium) - • • • • • • • • . 36

gracilis (Eucalymnatas) • . . . . . . . . 36

grandis (Coroplastos) . . . . . . . . . . 32

grandis (Dactylopius) - . . . . . . . . . 24

grundis ( nlvinaria) . . . . . . . . . 28

grвmais (Pzeudococcus). . . . . . . . . . 24

gregorius (Apioccecus) . . • . . . . . . 22

Gymnaspis . . . . . . . . . . . 44

Hemichonaspiś . . . . . . . . . . . 44

hemisphaericum (Lecanium) . . . . . . . . 41

hemisphaerica (Saissetia) . . . . . . . . . 41

hempeli (Polaeococcus). . . . . . . . . . 12

hesperidnm (Coccus) . . . . . . . . . . 36

hesperidum (Lecanuim) . • • . . . . . 36

Howardia. . . . . . . . . . . 43

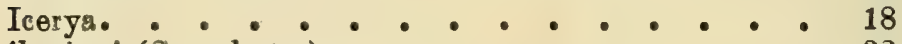

iheringi (Ceroplsstes) - . . . . . . . . . 32

inflatum (Mesolecsnium) . . . . . . . . . 37

infrequens (Lecanium) . . . . . . . . . . 41

infrequens (saissetia) . . . . . . . . . . 41

inga (Tachardi ) . . . . . . . . . . 26

insignis (Orthezia) . . . . . . . . . . . 18

insolens (Lecsnium) . . . . . . . . . 442

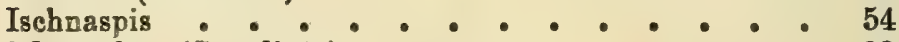

jaboticabse (Capulinia). . . . . . . . . . 23

jaboticabae (Lecanium) . . . . . . . . . 38

jaboticabze (Mesolecanium) . . . . . . . . 38

janeirensis (Ceroplastes) . . • . . • . . . 32

janeirensis (Odonaspis). . . . . . . . . 51

janeirensis (Odonaspis) (Aspidiotus) . . . . . . 51

Lachncdielia. . . . . . . . . . . . 24

lanigerum (Lecanium) . • • • . . . . 29

lanigerus (Malococcus) • • . . . . . . 29

latsniae (Aspidiotus) . . . . . . . . . . 42

Lecaniodisspis . . . . . . . . . . 20

Lepidosaphes. • • • . . . . • . . . . 53

Lichtensia • • • • • • • • • • • 29

lineare (Asterolecanium) . . . . . . . . . 19

linearis (Psendischnaspis) . . . . . . . . 51

longirostris (Mytilaspis) • . . . . . . . . 54

longirostris (1schnaspis) • . . . . . . . . 54

longispinus (Aspidiotus) . . . . . . . . . 48

lucida (Saissetis), 41 • . . . . . . . 60

lusiỏum (Mesolecenium), 67 • . . . . . . . 63

lucidus (Ceroplastes) . . . . . . . • 433 
maculata (Diaspis boiduvalli) • . • . . . . 43

magnolicida (Dactylopius). • . . . . . . . 25

magnolicida (Pseudococcus) • . . . . . . . 25

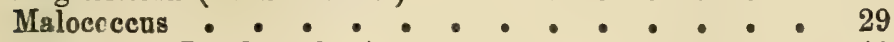

marianum (Paralecanium) . . . . . . . . . 40

msskelli (Morganella) . . • . . . . . 48

maskelli (Aspidiotus Morganella). . . . . . 48

mayteni (Lecanium) . . . . . . . . . . 38

msyteni (Mesolecanium) • • . . • • • . . 38

Mesolecanium • . • . . . . . . . . . 37

miliaris (Asterolecanium) • . . . : . : : 20

minor (Chionaspis) • • . . . . . . . . . 45

minor (Hemichionaspis) . . . . . . . . . 45

monile (Lecanium) . . . . . . . . . . . 39

monilis (Akermes) . . . . . . . . . . . 39

Monophlebinzo • . . . . . . . . . . . 17

Monophlebus . . . . . . . . . • . . $\$ 7$

moreirai (Aspidiotus) . . . . . . . . . . 42

Morgarella . • . . . . • . . . . . 48

multilobis (Dispidistis) • . . . . . . . . 52

Neolecanium. • • . • • • • • • • . . 39

nitens (Pserdokermes) • . . . . . . . . . 35

nitens (Pseudokermes) (Lecanium) . . . . . . 35

noacki (Plabinglisia) • • . . . . . . . 35

noacki (Pgeudoparlstoria) • . . . . . . . . 51

novresi (Ceroplastes) • . . . . . . . . . 33

obscurum (Lecanium) • • . . . . . . . . 28

obscurum (Mesolecanium) • • • • . . . . . 38

Odonarris . • . • • . . . . . . 51

olere (Kormes) . . . . . . . . . . . . 41

oleae (Leconium) • . . . . . . . . . . 41

oleae (Eaissetia) . : : : : : : . 41

oriertalis (Aspidiotus) : . . . . . . . . . 47

orlandi (Hemichionaspis) : $: \bullet^{\circ} \cdot \bullet^{\circ} \cdot \bullet^{\circ} 45$

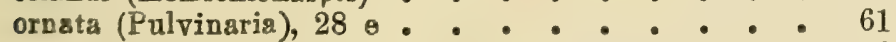

ornatum (Lecanium) • • • • • • . . 36

ornztum (Stictolecanium) - . . . . . . . 36

Ortheria : • • : : : : : : : 18

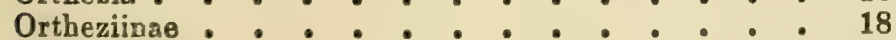

ovatus (Tectococcus) : . : . . . . . . 21

Palseocoscos. : : : : : : . . . 17

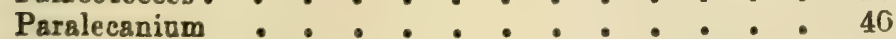

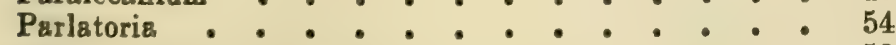

parlatarioides (Aspidiotus?) : . : . : . . 52

parłatorioides (Pseadoparlatoria) : . . . . . . 52

parva (Tachardia) . . . . . . . . . . 27

paulistus (Chryeomphalus). . . . . . . . . 50

paulistus (Chrysomphalus) (Aspidiotus) . . . . . 50

pentagcna (Aulacaspis). : . : • : • : 44

pentagona (Diaspis). . . . . . . . . . . 44 
perconvexum (Lecanium) - . • . . . . . . 39

perconvexum (Neolecanium) • • . . . . . $\quad 39$

perforatum (Lecanium). . . . . . . . . . 36

porforatus (Eucalymnatus). • • • . . • • 36

pergandii (Parlatoria) • . . . . . . . . . 54

perlonga (Lepidosaphes) . . . . . . . . . 53

perlonga (Mytilaspis) • . . . . . . . . . 53

perplexus (Eriococcus) . • . . . . . . . 21

personatus (Aspidiotus). •. . . . . . . . 50

personatus (Chrysomphalus) . . . . . . . . 50

Phenacocus •. . . . . . . . . . . 24

Pinnaspis. . . . . . . . . . . . . 45

pisai (Aspidiotus) . . . . . . . . . . . 47

Platinglisia • • • • • • • . • • . . 35

pomorum (Mytilaspis) . . . . . . . . . . 54

paraelonga (Orthezia) . . . . . . . . . . 19

proteus (Aspidiotus) • . . . . . . . . . 55

proteus (Parlatoria). . . . . . . . . . . 55

Protopulvinaria • . • . . . • . • . . 28

Pseułaonidis . . . . . . . . . . . . 48

Psoudichnaspis . . . . . . . . . . . 51

Pseudococcus. . . . . . . . . . . . 24

Pseudokermes . . . . . . . . . . . . 35

Psendoparlatoria. . . . . . . . . . . . 51

pseudosemen (Lecanium) . . . . . . . . . 38

pseudosemen (Mesolecsnium) • • . . . . . . 38

psidii (Coroplastes) . • • . . . . . . . 33

pulchella (Eulvinella) . . . . . . . . . . 28

Pulvingris • . . . . . . . . . . 27

Pulvinolia • . • • . . . . . . 28

purparens (Ceroplastes). . . . . . . . . . 33

pustulans (Asterolecanium). . . . . . . . . 20

rspax (Aspidiotus) . . . . . . . . . . . 48

raus (Ceroplastes) . . . . . . . . . . . 34

reticulats (Saissetis) . . . . . . , . . . 42

reticulatum (Lecanium). . . . . . . . . . 42

rhizophorae (Lecanium) . . . . . . . . . 38

rhizoph rae (Mesolecsnium) • • • • • • . . 38

Ripersia . . . . . . . . . . . . 25

rosae (Tashardia) . . . . . . . . . . . 27

rotundus (Ceroplastes) • . • . . . . . . . 34

rubra (Tachardia) • . . . . . . . . . 27

rugosa (Edwallia) . . . . . . . . . . 35

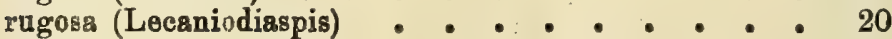

Saissetia . . . . . . . . . . . . 40

schrottkyi (Icerya) . . . . . . . . . . 18

scutiformis (Aspidiotus) • . . . . . . . . 50

scutiformis (Chrysomphalus) . . . . . . . . 50

secretus (Dactylopius) . . . . . . . . . . 25

secretus (Pseadococcus) . . . . . . . . . 25 
Selenaspidus

Stictolecənium • . • • • . . . • • • . 36

Stigmacoccus . • • • . . . . . . . . 17

Tachardia • . • . . . . . . . 26

Trcharc̉ine • . • • • • • • • • • . 26

tequarae (Ripersia), 25 в . . . . . . . . . 58

Tectococens . . . . . . . . . . . . 21

Tect pulvinaria . • • . • • . • • . 29

Termiticoccus • • • • • • • • • 26

trilobitiformis (Aspidiotus). . . . . . . . . 48

trilobitiformis (Pseudaonidia) . . . . . . . . 48

tuberculus (Solenococcus) . . . . . . . . . 21

ulmi (Coccus) • • • . • . . . . . . . 54

ulmi (Lepidosaphes). . . . . . . . . . . 54

urichi (Lecanium) . • • . . . . • . . . 39

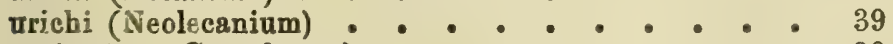

variegatus (Ceroplastes) : . . . . . . . . 39

Vinsonia . . . . . . . . . . . . . . 34

viridi (Lecanium) . . . . . . . . . . . 37

virides (Carpochloroides) : . . . . . . . . 22

viridis (Coceus) : . • • . . . . . . . . 37

zanthocxylum (Lecanium) . . . . . . . . 42

zanthos ylum (Saissetia) . . . . . . . 42 



\section{CATALOGOS}

DA

\section{FHŨX BR\%\%IIEIR\%}

EDITADOS

Museu Paulista

SÃo PAULO-BRAZIL

VOL. IV

As Chrysididas do Brazil

POR

ADOLPHO DUCKE

Entomologo do Museu, Goeldi - Pará

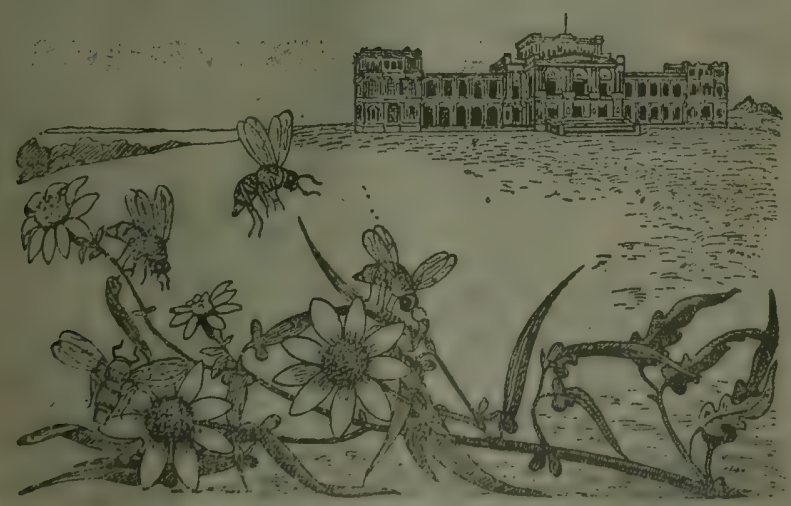

SÃo PAULO

Typ. do "Diario Official " 



\section{As Chirysididas do Brazil POR}

\section{ADOIPEIO DUCKF}

Entomologo do Museu Goeldi - Pará

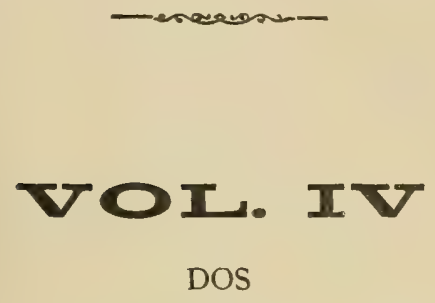

Catalogos da Fauna Brazileira EDITADOS PELO

\section{MUSEU PAULISTA}

SÃO PAULO - BRAZIL

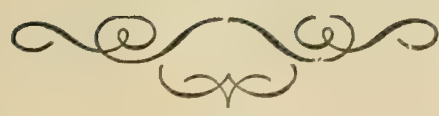

SÃo PAULO

Typ. do "Diario Official . 

E' com prazer que editamos como $4 .^{\circ}$ volume da serie dos nossos Catalogos da Fauna Brarileira o presente trabalho do nosso competente collega do Museu Goeldi do Pará. Tendo-se especializado ha longo tempo no estudo da interessante familia das Chrysididas, estamos certos de que a criteriosa revisão a que o sr. Adolpho Ducke sujeitou este grupo de hymenopteros, vem facilitar muito o respectivo estudo systematico.

Pela conveniencia apenas da uniformidade em nossos "Catalogos», demos caracter de subspecies ás fórmas quie o sr. A. Ducke considerava como variedades; não vae nisto modificação essencial nem de gráu nem cathegoria. $\mathrm{O}$ que o auctor considera variedade, corresponde perfeitamente ao que designamos como subspecie na nomenclatura trinominal; o mesmo verifica-se no arranjo que o auctor deu ás vespas sociaes, cujo catalogo publicaremos logo em seguida a este.

Das 80 especies brazileiras enumeradas neste catalogo, possuimos 46 nas collecções do Museu Paulista, material este que, tendo sido estudado primeiramente pelo sr. Visconde R. du Buysson do Museum d'Histoire Naturelle de Paris, foi depois tambem revisto cuidadosamente pelo sr. A. Ducke. No Estado de S. Paulo foram até agora observadas 2 I especies desta familia.

O Director do Museu Paulista

Di. Hermante von Ilerering 



\section{Cátalogo das Chrysididas do Brazil}

\section{Enumeração dos generos, das especies e das subspecies}

POR

\section{ADOLPHO DUCKE}

As Chrysididas são uma das familias bem estudadas dos hymenopteros, graças ás lindas côres metallicas que lhes attrahem a attenção dos colleccionadores e dos entomologistas, sobretudo no velho continente, onde poucos outros insectos se lhes comparam em belleza. Na fauna da America central e meridional, porém, tão primorosa em quasi todas as ordens de insectos, pela magnificencia das côres e variedade das formas, esta familia occupa um logar pouco saliente, por ser composta quasi exclusivamente de especies pouco vistosas, cuja côr predominante é um monotono verde, que só de quando em vez passa para um azul esverdeado ou para um violaceo escuro, com exclusão absoluta das fulgentes côres igneas e douradas que observarnos na maioria das especies do hemıspherio oriental.

Em 1889, Mocsáry enumerou em sua monographia $\mathbf{1 1}$ especies brazileiras; hoje conhecemos quasi o dobro, 80) especies, devendo-se attribuir este augrmento principalmente ás exploraçoses da fauna brazileira, feitas pelo Museu I'aulista no Sul e pelo Museu Paraense no norte do pai\% $\Lambda$ distribuiça actualmente averiguada das especies brazileiras es a seguinte: 
Antilhas; continente americano, dos Estados Unidos da America do Norte até a zona temperada austral . .

Continente americano, dos Estados Unidos da America do

Norte até a zona temperada austral . . . . . 6

Do Mexico ou da America central (exclusive Panamá) até

as partes medianas da Rep. Argentina. . . . . . 5

Do Mexico ou da America central (exclusive Panamá) até

a zona subtropical austral.

Do Mexico ou da America central (exclusive Panamá) até

a Amazonia.

Das Rep. ${ }^{a n}$ de Panamá, Colombia ou Venezuela até o tropico austral ou além. . . . . . . . . 4

Da Amazoria (inclusive Guyana) até as partes medianas da

Rep. Argentina . . . . . . . . . 4

Da Amazonia (inclusive Guyana) até a zona subtropical austral . . . . . . . . . . 3

Da Amazonia (inclusive Guyana) até a região tropical austral . . . . . . . . . . . . . 3 Amazonia e Guyana . . . . . . . . . . . . 7 Amazonia superior e inferior . . . . . . . . . 7 Amazonia superior. . . . . . . . . . . . . . 1 Amazonia inferior. . . . . . . . . . . . . 10 Região austral tropical e temperada. . . . . . . . 3

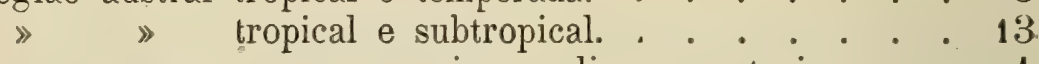
$\gg \gg$ e paizes andinos equatoriaes . . 1

5 especies são citadas dn Brazil, sem outras indicações; devemos suppol-as pertencentes á fauna do Brazil central ou meridional e certamente não á fauna amazonica, visto que mesmo emı collecções antigas os insectos amazonicos costumam ter a indicação «Pará» ou «Amazonas».

Das duas faunas principaes que existem no Brazil, a da Amazonia possue 27 especies de Chrysididas, a do Brazil central e meridional 17 especies (ou $2 ?$ incluindo as que são descriptas do Brazil sem outra indicação); 27 especies são communs a ambas as faunas; 4 são conhecidas do Sul do Brazil e da America central ou do Norte da America meridional, sem terem sido encontradas na Amazonia. A relativa riqueza da fauna amazonica torna-se patente na Anıazonia inferior, onde principalmente no limite das varzeas do valle alluvial do grande rio e das terras firmes ao Norte e ao Sul, a abundancia de 
Eumenididas, Apidas solitarios e Sphegidas farorece o desenvolvimento de Chrysididas, que são quasi todos parasitas de hymenopteros das familias citadas. A Amazonia superior, uniformemente coberta de florestas, é muito mais pobre em especies e ern individuos. A fauna da Guyana é uma continuação da Amazonia inferior, e esta prolonga-se para o Sul até as partes septentrionaes e centraes do Estado do Maranhão e, com relação a algumas especies, até o Piauhy. No sul do Maranhão e no Ceará apparecem, no meio d'uma fauna pobre, as primeiras especies da fauna meridional, a qual já se pode dizer bem conhecida nos Estados de Minas Geraes, São Paulo, Santa Catharina e Rio Grande do Sul, emquanto o Espirito Santo, a Bahia e os Estados situados ao Norte deste ultimo carecem ainda quasi totalmente de explorações. Sobre a fauna do Matto Grosso não sabemos quasi nada.

Se compararmos a distribuição das Chrysididas brazileiras com a das Vespidas sociaes, notamos logo, que entre as primeiras existem muitas especies de distribuição vastissima. Não poucas habitam tão bem as terras equatoriaes como as regiões temperadas e mesmo bastante frias do Norte e do Sul do continente.

As especies 5, 6, 18, 19, 20, 22, 36, 41, 44, 47, 50, 53, 54, 55, 73 não estão representadas nas collecções do Museu Paulista nem nas do Museu Paraense. Destas especies, onze foram descriptas uma unica vez e năo mais citadas por outros autores, sendo tres de Dahlbom, cinco de Mocsáry, uma de Brèthes e duas de Buysson. E' possivel, que algumas d'estas especies sejam synonymas de outras.

\section{LITERATURA}

(Das obras enumeradas no "Catalogus hymenopterorum» de Dalla Torre são citadas sómente aquellas que contèm dados importantes sobre a distribuiça geographica das especies).

1. Bischoff, II.: Die Chrysididen des $\mathrm{Kgl}$. zoologischen Museums zu Berlın. Mitleilungen zool Mus. Berlin IV. 1910 , p. $425-493$. 
2. Brèthes, J.: Contributions à l'étude des hymén. de l'Amérique du Sud et specialement de la Rép. Argentine : Les Chrysidides. Ann. Mus. Nac. Buenos Aires (3) I, 191)2 p ¿263-294.

3. BRÈthes, J.: Hinenópteros sudamericanos. Ibidem (3) IX, 1906, p. 1-13.

4. Bréthes, J.: Himenópteros de Mendoza y de San Luiz. Ibidem (3) X, 1909, p. 455-463.

5. Buysson, R. Du : Contribution aux Chrysidides du globe. Revue d'Entomologie X, 1891, p. 29 ss., XII, 1893, p. 245 ss., XVII, 1898, p. 125 ss., XIX, 1900, p. 125 ss., XXIII, 1904, p. 253 ss.

6. Buysson, R. Du : Étude des Chrysidides du Muséum de Paris. Ann. Soc. Entom. France L XVl, 1897, p. 518-580.

7. Bursson, R. DU: Catalogue des insectes hymén. de la fam. des Chrysidides du Mus. de Paris, Bull. Mus. d'hist. Nat. 1899 n. 4, p. 15!'-169.

8. Buysson, R. DU: Sur quelques Chrysidides du Musẻe de Vienne. Ann. naturh. Hofmuseum XVI, 1901, p. 97-! 04 .

9. Bursson, R. DU: Voyage de M. A. C. Jensen Haarup dans la région de Mendoza. Hyménoptères. Entomologiske Medelelser (2) III, 1906, p. 17-20.

10. Buysson, R. Du: Hyménoptères Ghrysidides de la région de Mendoza. Ibidem (*) IIII, 1908, p. 195-202.

11. Bursson, R. Du: Sur quelques parasites des Euménides. Revue d'Entom. 1908 p. 106-1C9.

12. Buysson, R. Dis: Vespides et Chrysidides, em: Embrik Strand, Hymenopterenfauna von Paraguay. Zoolog. Jahresber. XXIX, 191", p. Z31-241.

13. Buysson, R. Du: Hyménoptéres nouveaux ou peu connus. Revue d'Entom. XXVIII, 1910, p. 172-176.

14. Dalla Torre, C. Y. DE: Catalogus hymenopterorum, etc. VI, 1892. 
15. Ducke, A.: Beiträge z. Kenntnis d. geograph. Verbreitung d. Chrysididen ı. Beschreibung von drei neuen Arten. Zeitschr. system. Hymen. Dipter. I, 1901, p. $353-361$.

16. Ducre, A.: Eine neue südamerikanische Cleptes Art. lbidem II, 1902, p. 92-93.

17. Ducke, A.: Neue südamerikanische Chrysididen. Ibidem II, 1902, p. 97-101; III, 1903, p. 129-136, 226-232.

18. Duche, A.: Ein wenig bekanntes Chrysididengenus : Amisega Cam. Ibidem II, 1902, p. 141-144.

19. Ducke, A.: Neue Goldwespen von Pará. Ibidem II, 1902 p. 204-207.

20. Ducke, A.: Revisione dei Crisididi dello stato brasiliano del Pará. Bull. Soc. Entom. Ital. XXXVI, 1904, p. 13-48.

21. Ducke, A.: Supplemento alla Revisione dei Crisididi. Ibidem XXXVI, 1904, p. 9y-102.

22. Ducke, A.: Secondo supplemento alla Revisione dei Crisididi. Ibidem XXXVIII, 1906, p. 3-14.

23. Ducke, A.: Terzo supplemento alla Revisione dei Crisididi. Ibidem XLI, 1910, p. 89-115.

24. Ducke, A. : Contribution à la connaissance de la faune hyménoptérologique du Nord-Est du Brésil. Revue d'Entom. XXVI, 1907, p. 73-96; XXVII, 1908, p. $57-87$; XXVIII, 1910, p. 78-122.

25. Duche, A. : Contributions à la connaissance des hyménoptères des deux Amériques. Ibiden XXVII, 1908, p. $28-55$.

26. Kriechbaumer: Von I. K. II. Prinzessin Therese von Bayern in Südamer. resammelte Insekten. Berl. Entom. Zeitschr. XLV, 1900, p. 97-107

27. Mocsáry, A. : Monographia Clırysididarum orbis terrarum universi. 1889.

28. Mocsiry, A. : Additamentum primum ad monographiain Chrysididarum. Termiszetrajzi Füzeteck Xlí, 1890, p. 45-66. 
29. Mocsárx, A.: Additamentum secundum ad monographiam Chrysididarum: Ibidem XV, 1892, p. 213-240.

30. Mocsáry, A.: Species aliquot Chrysididarum novæ. Ibidem XXV, 1902, p. :339-349.

31. Mocsỉry, A.: Observatio de Clepte aurora. Annal. Mus. Nat. Hungar. II, 1904, p. 5€7-こ69.

32. Mocsảry, A.: Species Chrysididarum novæ. Ibidem IX, 1911, p. $443-474$.

33. Schulz, W. A.: Spolia hymenopterologica, 1906. 


\section{Familia CHRYSIDID $A$}

\section{SUB-FAMILIA CLEPTIN E}

\section{Genero A MEs}

\section{Amisega moesáryi (Durlie 1902)}

22, 23).

Distrib. (*) Guyana (21), Amazonia superior e inferior (20, Mus. Paul.: E. do Pará, Belém, Itaituba.

1." Amisega mocsáryi eyaniceps Ducke

Amisega cyaniceps, Ducke 1910.

Distrib. Amazonia superior (23).

Mus. Paul. E. do Amazonas, Teffé.

2. Amisega xeneiceps Ducke 1903.

Distrib. Guyana (21), Amazonia sup. e inferior (20, 22). Mus Paul. E. do Pará, Obidos.

2. Genero De르롤 Mocsáry, 1890.

Pseudepyris Ducke 1902.

Parachrysis Kieffer 1909 (Annal. Soc. Ent. France LXXVIII p. 287).

\section{Adelphe paradoxa Ducke}

Pseudepyris paradoxa Ducke 1902. Adelphe parridoxa Ducke 1910.

Distrib. Grayana (21), Arnazonia sup. e inferior ( 20 , 23). Mus. Paul. E. do Pará, Belém.

(*) Ов numero ant-e purenthosis indicam \&s ob as citadas; $(A)$ rofere-se to ar pendice no fim deste cateloge. 


\section{Adelphe flavipes Ducke}

Pseudepyris flavipes Ducke 1903.

Adelphe flavipes Ducke 1910.

Parachrysis metallica Kieffer 1909 (Annal. Soc. Ent. France LXXVIII, p. 288).

Distrib.: Amazonia superior e inferior (20, 22).

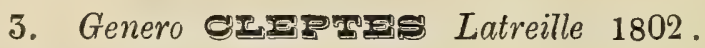

Diplolepis Fabricius 1804.

Cleptidea Mocsáry 1904.

5. Cleptes fasciatus Dalman 1823.

Cleptidea fasciata Mocsảry 1904.

Distrib. : «Brazil» (27).

6. Cleptes xanthomelas Mocsáry 1889.

Cleptidea xanthomelcena Mocsáry 1904.

Distrib. : Santa Catharina (27, :33).

7. Cleptes mutilloides Ducke 1902

Cleptidea mutilloides Mocsáry 1904.

Distrib.: Amazonia inferior, Belém.

Mus. Paul.: E. do Pará, Belém.

7. aleptes mutilloides minor $n$. subsp. (A).

Distrib.: E. do Pará, Faro.

8. Cleptes aurora $F$. Smith 1874.

Cleptidea aurora Mocsáry 1904.

Distrib.: Amazonia superior (22, 2\%); Bolivia amazonica subandina ( 31 ).

8. Cleptes aurora dubuyssoni $n$. n. (A).

Cieptes buyssoni Ducke 1904 nec Semenow 1891. Distrib. : Guyana (21). 


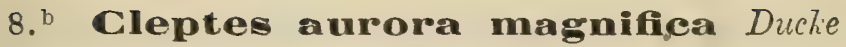

Cleptes magnificus Ducke 1904.

Distrib.: Guyana (21, 23) Amazonia superior (22).

8. Cleptos aurora robustior $n$. subsp. (A).

Distrib.: Norte do baixo Amazonas, Faro.

Mus. Paul.: E. do Pará, Faro.

SUB-FAMILIA GHRYSIDIN E ou CHR YSIDIN F GENUINA

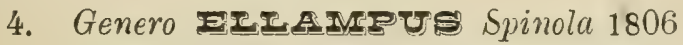

Umalus Dahlbom 1854, nec Jurine.

Sut-genero rogezUs Foerster 1853

9. Ellampus gayi Spinola 1853 .

Ellampus (Notozus) gayi Mocsáry 1889.

Ellampus (Notozus) cequinoctialis Ducke 1201 (aberr.) Distrib. : Amazonia inferior ('20); Ceará (23); Rep. Argentina: Chaco (5), Mendoza (1,9); Chile (7).

Mus. Paul.: Rep. Argentina.

10. Ellampus pulchricollis Ducke 1910.

Distrib. : Minas-Geraes, Barbacena (23).

Sub-genero Fore로요 Mocsúry 1890 (?), Buysson 1901

\section{Ellampus huberi Ducke 1901.}

Holophrys iridescens Buysson 1901 (ou Ellampus iridescens Norton?).

Ellampus (Philoctetes) minutissimms Brithes 1902.

Distrib.: Amazonia superior e inferior (20, 22, 23), Minas-Geraes (23), Rio Grande do Sul (S), Rep. Argentina: Mendoza (9).

Mus. Paul.: E. do Pará, Obidos.

12. Ellampus albolimbatus Duclie 1908.

Distrib. : IIonduras britannico (25), Amazonia inferior (23). 


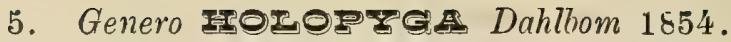
Sub.genero Hozopяas. str.

\section{Holopyga paraensis Ducke}

Ellampus paraensis Ducke 1901.

Holopyga paraensis Ducke 1910.

- - Buysson 1910.

Distrib.: E. do Pará: Oyapoc (21), Belém (20), Bragança $(A)$.

Mus. Paul.: E. do Pará, Oyapoc.

14. Lolopyga kohli Buysson 1901 .

Holopyga kohli Buysson 1910.

Holopyga pallidolimbata Ducke 1903.

Distrib. : Amazonia inferior (20), «Brazil» (8).

\section{4. ${ }^{2}$ Holopyga kohli piliventris Ducke.}

Holopyga piliventris Ducke 1907.

Holopyga kohli var. piliventris Buysson 1910.

Distrib.: Maranhão e Ceará (23).

Mus. Paul.: E. do Maranhão, Codó.

15. Holopyga lazulina Dahlbom 1854 .

Holopyga la:ulina Mocsáry 1889.

- $\quad$ - Buysson 1910.

Distrib. : «Brazil» (27); Rep. Argentina : Buenos-Aires e Mendoza (27), Pampa central (3); Chile (7, 4).

16. Holopyga dohrni Dahlbom 1854.

Holopyga ventralis Mocsảry 1889 ex-parte (não Hedychrum ventrale Say).

Distrib. : E. U. da America: Baltimore (6) ; Haiti (6) ; Amazonia inferior (20); Maranhão (23); Ceará (23); Rep. Argentina : Mendoza (1, 10).

Mus. Paul.: E. do Pará, Faro.

17. Holopyga iheringi Buysson 1901.

Distrib.: Colombia (5), E. de São Paulo, Rio Grande do Sul (8), Rep. Argentina: Chaco (13).

Mus. Paul.: E. de São Paulo, Jundiahy. 


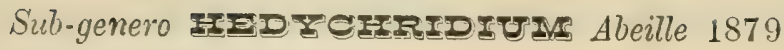

\section{Holopyga andréi (Mocs.)}

Chyrisis andrëi Mocsảry 1889.

Hedychridium andréi Buysson 191().

Distrib.: Bahia (1), Paraguay (12).

19. Holopyga torosa Mocsiry 1889.

Distrib. : «Brazil» (27).

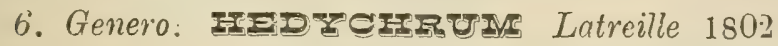

20. Hedychrum brasilianum Dahlbom 1852.

Distrib.: «Brazil» (27)

21. Hedychrum neotropicum Mocsíry 1898.

Distrib.: Mexico ( 7 ). Amazonia superior e inferior (20, 23), Maranhão (23), Ceará (23), E. do Rio de Janeiro (27), R. Argentina : Mendoza (1, 10).

Mus. Paul.: E. do Pará, Alemquer.

7. Genero: CIIRSIS Linné 1761 (A.)

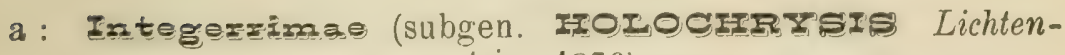
stein 1876)

22. Chrysis reticulata Mocsáry 1911.

Distrib. : Santa Catharina (32)

b: Inaequaleg (subgen GOIVOCIRTÐIs Lichtenstein 1876)

2.5. Chrysis iheringi Buysson 1904.

Distrib. : São Paulo

Mus. Paul. : São Paulo. 


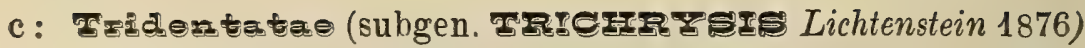

\section{Chrysis silvestri Dur:ke}

Chrysogona silvestri Ducke 1904.

Chrysis silvestri Ducke 1910.

Distrib. : Amazonia superior e inferior (20, 22, 23).

\section{Chrysis armata (Mocs.)}

Chysogona armata Mocsảry 1889.

Chrysis armata Ducke 1910.

Chrysogona saussurei Mocsáry 1889) (nec Chrysis saussurei Chevr.)

Chrysis ritsemae Mocsáry 1889.

Chrysis schrottkyi Brèthes 1902.

Distrib. : Mexico (27), Colombia (1), Guyana (1, 27), Amazonia superior e inferior (20, 23), Maranhão (23), Bahia (2), Minas Geraes (23), E. do Rio de Janeiro (2, 2T), São Paulo (2), Rio Grande do Sul (8), Rep. Argentina: Mendoza (10). ranga.

Mus. Paul.: E. do Pará, Obidos; E. de São Paulo, Ypi-

26. Chrysis erotonis Ducke 1906.

Distrib. : Lado Norte do baixo Amazonas (22).

Mus. Paul.: E. do Parả, Obidos.

27. Chrysis truncatella Dahlbom 1834.

Chrysis truncatella Mocsàry 1889.

Distrib.: Amazonia inferior (20, 23; Santa Catharina 1, (27); Bolivia: Tarija (1).

28. Chrysis brasiliama Guèrin 1842.

Chrysis brasiliana Mocsáry 1889.

Chrysis producta Brullè 1846.

Distrib.: Mexico (6), Amazoria inferior (23), Minas Geraes (6), Rio de Janeiro (27), Santa Ciatharina (27), Rep. Argentina: San Juan, Buenos Aires e Mendoza (2).

Mus. Paul. : E. do Pará, Obidos. 
29. Chrysis tridens (Lep. et Serv.)

Pyria tridens Lepeletier et Serville 1825.

Chrysis tridens Brulle 1846.

- — Mocsáry 1889.

Chrysis truncata Guérin 1842.

Chrysis mexicana Guérin 184?.

Chrysis tridentata Dahibom 1845.

Chrysis olivieri Brullé 1846.

Chrysis unicolor Brullé 1846.

Chrysis parvula Dahlbom 1854 (ou F.?)

Chrysis virens Gresson 1865.

Chrysis brullei Abeille 1879.

Distrib.: E. U. da America (1, 5, 6, 27), Mexico (1, 27), Guatemala (27), Nicaragua (27), Panamá (27), Guyana (5,) Brazil ? (27), R. Argentina: Santa Fé (6).

30. Chrysis mathani $n$. n. (A.)

Chrysis mutica Ducke 1906, nec Foerster 1853.

Distrib. : Amazonia superior e inferior (22).

31. Chrysis mucromata Brullé 1846.

Chrysis mucronata Mocsáry 1889.

Chrysis lunigera Dahlbom 1854.

Chrysis selenia Costa 1864.

\section{$31 .{ }^{2}$ Chrysis mucromata aliena Mucsiry.}

Chrysis aliena Mocsåry 1889.

Distrab. da firma typica esulsp. : E. IT. da America (1), California meridional (27), Mexico (1, 7, 1:2), Costa lica (A.), Panami (5), Cosombia (1, 5), Venezucla (7), Amazonia

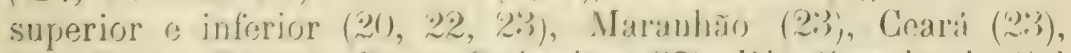
São Panlo, P'arani, Santa Catharina (207), lice Cirande do Sul (8), I'arasuay (1, 12), Mep. Areentira: Oran, Mendoza (10).

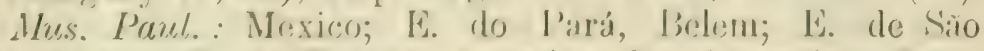
Paulo, Itatiba; Ej. Io P'arani, Antonina; R. Argentina, Oran. 
32. Chrysis trianguliferea Mocsáry 1890.

Distrib.: Guayana (1), Amazonia inferior (20, 22, 23), Piauby (28).

Mus. Paul. : E.do Pará, Obidos.

33. Chreysis amazonica Mocsáry 8891

Distrib. : Baixo Amazonas (27), Rio Negro (22).

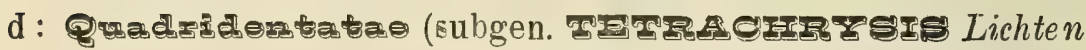
stein 1876)

34. Chrysis cameroni Buysson 1900.

Chrysis duckei Mocsáry 1902.

Distr b. : Amazonia superior e inferior (20, 23), Bahia (5). Mus. Paul.: E. do Pará, Obidos.

35. Chrysis punctatissima Spinola 1840.

Chrysis punctatissima Mocsáry 1889.

Chrysis fasciata Fabricius 1804 (não Olivier).

- $\quad$ - Dahlbom 1845, 1854.

Chrysis carina Brullẻ 1846.

Chrysis guedesi Ducke 1903.

Chrysis lecointei Ducke 1906 (aberr.)

Distrib.: Mexico (7, 2)i) Guatemala (27), Panamá (27), Guyana (1, 7, 27), Amazonia inferior (20, 2\%), Pernambuco (27), Rio de Janeiro (1), São Paulo, Paraná (27), Santa Catharina (1), Rio Grande do Sul (8), Paraguay (8), Puna boliviana (26), Sudeste da Bolivia (6), R. Argentina e Uruguay, até Mendoza (1,9), Buenos Aires $(1,27)$ e Montevidéo (27). «Chile» $(A)$.

Mus. Paul.: Goyaz; E. de São Paulo; E. de Santa Catharina, Golonia Hansa; Est. do Rio Grande do Sul; R Argentina, Mendoza.

36. Chrysis longirostris Gribodo 1879.

Chrysis longirostris Mocsáry 1889. quil (5).

Distrib.: Minas Geraes (27), Goyaz (5), Equador : Guaya- 
37. Chrysis distinctissima Dahlbum 1854.

Chrysis distinctissima Mocsáry 188 .

Chrysis fasciata Spinola 1840 (não Olivier).

Chrysis intricans Brèthes 1902 (não Spinola).

Distrib.: Nicaragua (A), Guyana (27), Equador (27), Amazonia inferior (20), Ceará (23), E. do Rio de Janeiro (2), São Paulo, Paraguay (3), Rep. Argentina até Buenos Aires (3) e Mendoza (3, 9, 27).

Mus. Paul.: E. do Pará, Ubidos; E. de São Paulo, Ypiranga.

\section{Chrysis carinulata Mccsáry 1889}

Distrib. : Amazonia superior e inferior (22, 23), Piauhy (27), Goyaz (5), Rio Grande do Sul (8). Mus. Paul.: E. do Pará, Obidos.

39. Chrysis brachypyga Ducke 1910.

Distrib.: Baixo Amazonas, Obidos (23).

40. Chrysis exciuata Brullé 1846.

Chrysis diana Mocsáry 1889.

Distrib. : E. U. da America: Texas (1); Guyana (1); Amazonia inferior (20); Piauhy (27); Minas Geraes (23); São Pau10, Rio Grande do Sul (8); Paraguay (1, 12); R. Argentina: Mendoza (1).

Mus. Paul.: E. de São Paulo, Ypiranga, Campinas.

\section{Chrysis fraterna Mucsúry 1889.}

Distrib. : Santa Catharina (27).

42. Chrysis peracuta Aaron 1885.

Chrysis peracuta Mocsáry 1884 (exclusive o synony:no) Distrit.: E. U. da America: 'Tenesse e Texas (27); Mexico (11); Amazonas inferior (23); Săo L’aulo.

Mus. Paul. : São Paulo. 
43. Chrysis myops Buysson 1904.

Distrib. : Minas Geraes (23); R. Argentina: Tucuman (5).

44. Chrysis goyasensis Buysson 1904.

Distrib. : Goyaz (5).

45. Chrysis lagopus Buysson 189 !.

Distrib. : R. Argentina, Mendoza $(9,10)$.

45." Chrysis lagopus ancilla Buysson 1891.

Chrysis ancilla Buykon 1897.

Chrysis lagopus var. ancilla Buysson 1908.

Distrib. : Minas Geraes (23), S. Paulo (1), R. Argentina até Mendoza $(2,9,10)$ ə Chubut (2).

Mus. Paul: R. Argentina, Mendoza.

46. Chrysis morosa Buysion 1901.

Distrib. : Amazonia superior: Teffé (25); R. Argentina • Mendoza (10).

47. Chrysis dolosa Buysson 1898.

Distrib. : Mexico (5), Venezuela (7); R. Argentina: Cihaco (5).-Existirá certamente no Brazil.

48. Chrysis braeiliensis Brulle 1846.

Chrysis chlorata Mocsảry 1889.

Distrb. : Minas Geraes (6,23), S. Paulo, Rio Grande do Sul (8), R. Argentina: Mendoza (9).

Mus. Paul. : E. de S. Paulo, Ypiranga.

49. Chrysis cavifroms Brullé 1846.

Chrysis cavifrons Buysson 1897.

Distrib. : Minas Geraes (2:3), S. Paulo, R. Argentina : Corrientes (6), Mendoza (10).

Mus. Paul. : E. de S. Paulo, Ypiranga, Jundiahy. 
50. Chrysis inclyta Mocsáry, 1689.

Distrib. : Minas Geraes, Lagôa Santa (27).

51. Chrysis sampaioi Ducke 1910.

Distrib. : Baixo Amazonas, Obiulos (23).

52. Chrysis nisseri Dalhtrm 1845.

Chrysis propinqua Mocsáry 1889.

Nistrib. : Antilhas: S. Vicente (1); E. U. da America : Pennsylvania (1), Arizona (7), Texas (12); Mexico (7, 27); Colombia ( $\%, 2 \%)$; Verezuela (5); Guyana (1, 12); Amazonia superior e inferior (20, 22, 23; Bolivia (1); Minas Geraes (23); Săo Paulo; Santa Catharina (27); Paraguay (1, 1:2); P. Argentina até Mendoza (1, 12) e Buenos Aires (2).

Mus. Paul.: E. de S. Paulo, Ypiranga, Jundiahy; R Argentina, Mendoza.

\section{Chrysis panamensis Cameron 1888.}

Distrib. : Panamá (27), Rio de Janeiro (5).

54. Chrysis imbecilla Mocsúry 1889.

Distrib. : Santa Catharina (27).

05. Chrysis mattogrossensis Biéthes 1902.

Distrib.: Matto Grosso (2).

56. Chrysis alfkeni Ducke

Chrysogona alfkeni Ducke 1902.

Chrysis alfkeni Ducke 1910.

Jiscril, : IInnluras britannice, (23); Amazonia superior (23) e inferior (20).

Mus. Paul.: E. do Pará, Itaituba.

37. Chryain leucophrom Mockiny 1889.

Distrib. : Amazonia superior (23, 27). 


\section{Chrysis spingera Spinola 1840}

Chrysis spingera Mocsáry 1889.

Chrysis leucostigma Mocsáry 1889 (aberr).

Chrysis nitens Ducke 1906 (aberr.)

Distrib. : Mexico (7), Guyana (27). Amazonia superior e inferior (22, 23, 27), S. Paulo, Rio Grande do Sul (8), Paraguay (12).

\section{Chrysis imperforata Gribodo 1879.}

Chrysis spingera var. imperforata Mocsáry 1889.

Distrib.: Guyana (21, 27), Amazonia superior e inferior (20, 22, 2:3), Bahia (1).

Mus. Paul. : E. do Pará, Posto brazileiro no Oyapoc.

60. Chrysis brevispina Ducke 1910.

Distrib.: Baixo Amazonas, Obidos.

Mus. Paul.: E. do Pará, Obidos.

61. Chrysis marginalis Brullé 1846.

Chrysis marginalis Mocsáry 1889.

Chrysis ellampoides Ducke 1902.

Distrib. : Guyana (27), Amazonia inferior (20, 27). Mus. Paul.: E. do Pará, Obidos.

62. Chrysis leucocheiloides Ducke 1903.

Distrib.: Amazonia superior e inferior (20, 22, 23.) Mus. Paul. : E. do Pará, Obidos.

63. Chrysis leucocheila Mocsáry 1889.

Distrib. : Mexico (27), Colombia (1, 7), Guyana (21), Amazonia superior e inferior (20, 23), Ceará (23), Minas Geraes (623), E. de Rio de Janeiro (27). Rio Grande do Sul (8), Paraguay, (1); Bolivia (1).

Mus. Paul.: E. do Pará, Obidos.

64 Chrysis ypiranguensis Buysson 1904.

Distrib. : São Paulo, Ypiranga.

Mus, Paul. : E. de São Paulo, Ypiranga. 
65. Chrysis postica Brullé 1846.

Chrysis albomarginata Mocsảry 1889.

Distrib. : Colombia (5), Venezuela (5), Amazonia inferior (20, 22,) Minas Geraes (6, 23). Rio de Janeiro (27), São Paulo, Rio Grande do Sul (\$), Paraguay (1).

Mus. Paul. E. de S. Paulo, Franca.

66. Chrysis iparaensis Ducke 1903.

Distrib. : Amazonia superior e inferior (20, 22). Mus. Paul. : E do Pará, Belém.

67. Chrysis arôgentina Bréthes 1906.

Chrysis argentina Ducke 1910.

Distrib. : Maranhão Ceará, Minas Geraes (23), Rep. Argentina: Misiones (7).

68. Chrysis glabriceps Ducke 1903.

Distrib.: Amazonia inferior (20, 22, 23), Ceará (23), Minas Geraes (23), Rep. Argentina: Mendoza (10).

Mus. Paul.: E. do Pará, Obidos.

69. Chrysis inseriata Mocsáry 1902.

Distrib. : Belem do Pará (30).

70. Chrysis confusa Ducke $\mathbf{1 9 1 0}$.

Distrib. : Amazonia superior e inferior (23).

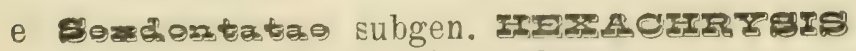

Lichterstein 1876.

71. Chrysis fabricii Mocsáry $1882,1889$.

Chrysis sexdentata Fabricius 1798, não Christ. Dahlbom 1854.

Chrysis dromeda Buysson 1897 (aberr.)

Distrib.: E. U. da America: ('xcorgia e Tenessee ('27), Mexico (1); Guyana (27); Amazonia inferior ( 2 ?); l'iauhy (27), Minas Geraes (5), E. do Rio de Janeiro (27), S. I'aulo, Rio Grande 
do Sul (1, 27, 8), Paraguay (1, 3, 8), Bolivia (1), R. Argentina: Chaco (5), Mendoza (2).

Mus. Paul.: E. de São Paulo, Ypiranga.

72. Chrysis smidti Dahlbom 1854 .

Chrysis smidti Mocsåry 1989.

Chrysis anceps Gribodo 1879.

Distrab.: Nicaragua (1), Guyana (1, 7, 21), Amazonia inferior (20, 23), Rio de Janeiro (6), S. Paulo, Paraguay (1.) Mus. Paul. : E. de S. Paulo.

73. Chrysis aequinoctialis Dahllom $180 ّ 4$.

Distrib. : "Brazil»(2i).

74. Chrysis frieseana Ducke 1902.

Distrib. : Amazonia superior e inferior (20, 23).

Mus. Paul. : E. do Pará, Obidos.

75. Chrysis affinissima Ducke $190 \overline{3}$.

Distrib. Belem do Pará (20).

76. Chrysis genbergi Dahlbom 1854.

Chrysis genbergi Mocsảry 1889.

Distrib. : Guyana (12), Amazonia superior e inferior (20, 23), Maranhão (23), Goyaz, Minas Geraes (23), E. do Rio de Janeiro (27), S. Paulo, Paraná (6), Rio Grande do Sul (8), Paraguay (12), Bolivia Oriental (1).

Mus. Paul.: E. do Pará, Obidos; E. de Minas Geraes, Goyaz, E. de S. Faulo, Ypiranga, Itatiba, Franca.

77. Chrysis lateralis Brullé 1846.

Chrysis lateralis Mocsáry 1889.

Distrib.: E. U. da America: Texas (1); Colombia (27); Guyana (1); Amazonia inferior (20, 23); Maranhão (23); Piauhy (27); Geará (23); Minas Geraes (23); S. Paulo, Paraná (27); R. Argentina: Mendoza (9); Buenos Aires (2.)

Mus. Paul.: E. de S. Paulo, Ypiranga, Jundiahy. 
77. Chryois Iateralis obidensis Ducke.

Dhrysis abidensis Ducke 1903.

Distrib. : Baixo Amazonas (23).

Mus Paul.: E. do Parź, Obidos.

77.b Chrysis lateralis goeldii Ducke.

Chrysis goeldii Ducke 1906.

Distrib. Baixo Amazonas (23!.

Mus. Paul.: E. do Pará, Obidos.

78 Chrysis bisuleata Ducke 1902.

Distrib.: Belem do Pará (20).

79. Chrysis Iongiventris Duike 1906.

Distrib.: Baixo Amazonas, Obidos. Mus. Paul. E, do Pará, Obidos.

80. Chrysis klugi Dahlbom 1854.

Chrysis klugi Moısảry 1889.

Distrib.: Venezuela (27), Guyana (i), Amazonia inferior (20), Piauhy (27), S. Paulo, Santa Catharina (27), Rep. Argentina: Misiones Chaco Mendoza (2).

Mus. Paul. : E. de S. Paulo, Victoria. 



\section{INDICE ALPHABET!CO DOS GENEROS E DAS ESPECIES}

Adelphe $I I$

aeneiceps 2

aequinoctiales $8,7.3$

affinissima 75 ,

albolimbatus 12

albomarginata 65

alfkeni 56

aliena $31^{\mathrm{a}}$

amazonica 3.3

Amisega $I$

anceps 72

ancilla $45^{\mathrm{a}}$

andréi 18

argentina 67

armata 25

aurora 8

bisulcata 78

brachipyga 39

brasiliensis 48

brasiliana 28

brasilianum 20

brevispina 60

brulléi 29

buyssoni 8

cameroni 34

carina 35

carinulata 38

cavifrons 49

chlorata 48

Chrysis VII

Chrysogona VII

Cleptes 111

Cleptidea III confusa 70

crotonis 26

cyaniceps $1 .{ }^{\mathrm{a}}$

diana 40

Diplolepis III

distinctissima 37

dohrni 16

dolosa 47

dromeda 71

duckei 34

dubuyssoni $8 .^{\mathrm{a}}$

ellampoides 61

Ellampus $1 \mathrm{~V}$

excavata 40

fabricii 71

fasciata (us) 5, 35, 37

flavipes 4

fraterna 41

frieseana 74

gayi 9

glabriceps 68

goeldii $77^{\mathrm{b}}$

goyasensis 44

guedesi 35

Hedychridium V

Hedychrum VI

Holophrys IV

Holopyga $V$

huberi 11

iheringi 17,23

imbecilla 54

imperforata 59

inclyta 50 


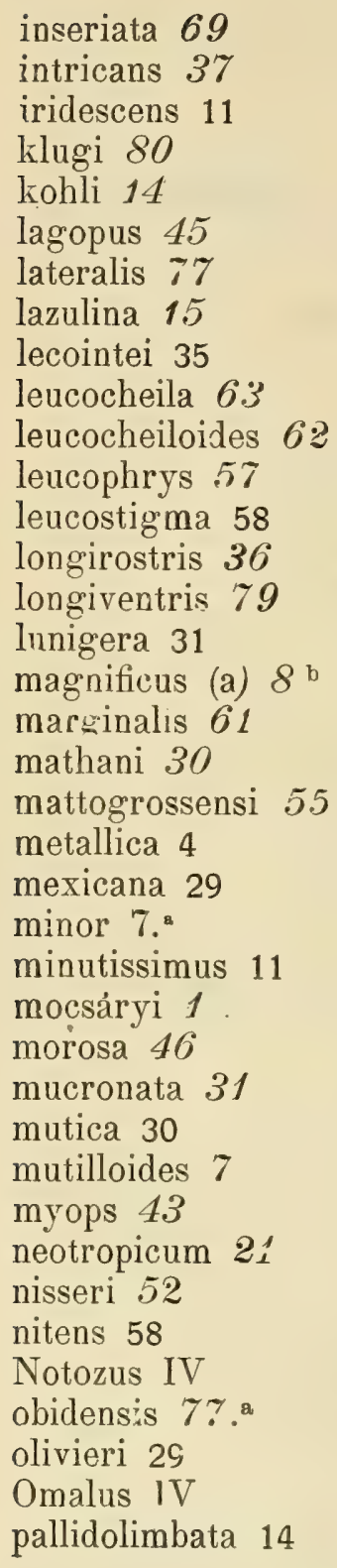

panamensis 5.3

Parachrysis II paradoxa 3 


\section{APPENDICE}

Notas sobre algumas especies brazileiras de Chrysididas

Subfamilia Cleptinae: Bischoff (o. c. 1) elimina das chrysididas a subfamilia das Cleptinas para collocal-a na familia das Bethylidas, porem eu prefiro (mórmente tratando-se de um catalogo) não abandonar o systema até agora seguido por todos os especialistas, emquanto não tiver chegado a uma conclusão segura nesta questão. E' certo, que os machos do genero Adelphe indicam uma transição tão evidente entre as Cleptinas e as Bethylidas, como não conhecemos nenhuma entre as Cleptinas e as Chrysididas genuinas. O que é certo é sómente que devemos separar as Cleptinas (ás quaes indubitavelmente pertencem as Amiseginas e Adelphinas de Mocsáry) de todas as outras Chrysididas, dividindo a familia em duas subfamilias de egual valor. As subfamilias Ellampinae, Hedychrinae, etc. dos autores não têm, ao meu vêr, razão de existir, por terem os generos que as compunham, estreito parentesco com o genero Chrysis.

7. Cleptes mutilloides mINoR n. subsp.: A forma genuina differt: corpore minore $\left(4^{1 / 2}-5\right.$ millimetro longo): orbitarum internarum maculis of nullis, $o^{x}$ obsoletis; pronoto \& $0^{x}$ marginibus exceptis nigroaeno. Faro (E. Pará).

A forma typica da especie é sómente conhecida de Belem do Pará.

8. Cleptes aurora Sm. - Variavel no tamanho do corpo e na intensidade da cor metallica. Conheço desta especie 4 formas distintas:

A : Cleptes aurora dubuyssoni n. n. (Cleptes buyssoni Ducke, nec Semenow): Corpus gracile, $6-6^{1 / 2}$ millm. longum, sat obscure viridiaeneum, segmento mediano et abdomine (picturis albis exceptis) nigro 
aeneis, mesonoto obscure purpureoviolaceo, scutello postscutelloque rufis, hoc ultimo gibbo, superne leniter emarginato, pedibus nigris cyanescentibus solum coxis trochanteribusque infra sordide albidis. Oyapoc.

B : Cleptes aurora, forma typica: Praecedenti parum robustior, $6^{1 / 2}-8$ millim. longus, laete viridiaeneus vel viridicyaneis, segmento mediano et abdomine cyaneis, scutello rufo, mesonoto et postscutello laete purpureoviolaceis, hoc ultimo vix magis gibbotuberculato quam in subspecie praecedente. Pedes cyanei, etiam metatarsis intermediis et posticis albis. - Amazonia superior até a Bolivia subandina.

C: Cleptes aurora Robustion n. subsp.: Maior (7-9 millim. longus) et multum robustior: color ut in forma praecedente, sed saturatior, thorace laete viridicyaneo, segmento dorsali $1 .^{\circ}$ fere toto albo; segmenti mediani dentibus longioribus. Scutellum multum magis convexum quam in varietatibus praecedentibus; postscutellum multum longius, mucroniforme, profundius excisum. - Faro (parte noroeste do E. do Pará).

D : Cleptes aurora magnifica Ducke : Ut subsp. robustior, sed corpore saturate cyaneo, scutello violaceo, mesonoto postscutelloque concolore. - Guyana, Amazonia superior.

13. Holopyga paraensis Ducke: Tambem encontrada em Bragança, na parte sudoeste do litoral do E. do Pará.

VII. genero, Chrysis L. - Ainda não se conseguiu uma classificação natural das numerosas especies deste genero, sendo artificial a que até agora foi seguida pelos autores e que eu tambem sigo por falta de outra melhor. Nas especies neotropicaes, as Tridentadas constituem um grupo de parentesco natural, porem entre as Quadridentadas e Sexdentadas encontram-se especies de muita affinidade.

30. Chrysis mathani n. n. - Estando o nome mutica já occupado por uma especie descripta por Foerster, dou á minha especie o nome do meu antigo companheiro de excursões em Obidos, o snr. M. Hue de Mathan, muito conhecido pelas 
collecções de insectos por elle reunidas na Amazonia e nos paizes andinos.

31. Chrysis mucronata Brullè. - Vi na collecção do prof. Baker um exemplar de Costa Rica.

35. Chrysis punctatissima Spinola. - Na o. c. 23 citei este especie como occorendo no Chile, porem agora não encontro o autor, em que me baseei, pelo que me restam duvidas sobre a indicação. O Sceliphron figulus Dahlb., da qual esta Chrysis é parasita, existe no Chile.

36. Chrysis distinctissima Dahlb. - Nicaragua: Managua (collecção Baker).

Já estava terminada a composição deste catalogo, quando recebi um recente trabalho do snr. A. Mocsáry: Species Chrysididarum novae, III ; Ann. Mus. Nat. Hungarici X, 1912, p. 549 - 592, com a descripção das seguintes especies brazileiras:

\section{Quadridentatae:}

Chrysis adolphi Mocs. obra cit. p. 574, Est. do Pará: Obidos; Obidos .

Chrysis florisomnis Mocs. ibidem, p. 576, Est. do Pará :

Chrysis subtruncata Mocs. ibidem, p. 577, Est. Minas Geraes ;

Chrysis deuteroleuca Mocs. ibidem, p. 578 Est. Rio Grande do Sul: Santa Cruz.

\section{ERRATA}

P. 7 linha 1: solitarias (em vez de os)

P. 7 : : todas (em vez de os)

P. $7>6: d a$ da Amazonia (em vez de

da Amazonia)

?. 8 n. 12: Jahrbuecher (em vez de Jahresber.)

P. 11. especie n. 1 : supprima-se o parenthesis em que esta o nome do auctor.

P. 12 linha 7 : Chrysididae genuinae (em vez de Chrysidinae genuinae).
P. 13 linha 9: Omalus (em vez Umalus)

P. 16 especie n. 24: Chr, s Ivestrii (em vez de silvestri

P. 18 especie $n .33: 1889$ (em vez de 8891)

P. 22 especie n. 58 : spinigera (em vez de spingera

P. 27 linha 5: aequinoctialis, 9 (e. v. de 8 )

r. $282^{\circ}$ col., linha 16 : Pyria (em vez de Pyrix)

P. $282^{\text {n }}$ col., penultima lin. xanthomelas. 
